\title{
MIRROR SYMMETRY FOR LOG CALABI-YAU SURFACES I
}

\author{
MARK GROSS, PAUL HACKING, AND SEAN KEEL
}

\begin{abstract}
We give a canonical synthetic construction of the mirror family to pairs $(Y, D)$ where $Y$ is a smooth projective surface and $D$ is an anti-canonical cycle of rational curves. This mirror family is constructed as the spectrum of an explicit algebra structure on a vector space with canonical basis and multiplication rule defined in terms of counts of rational curves on $Y$ meeting $D$ in a single point. The elements of the canonical basis are called theta functions. Their construction depends crucially on the Gromov-Witten theory of the pair $(Y, D)$.
\end{abstract}

\section{Contents}

Introduction

0.1. The main theorems

0.2. The symplectic heuristic

0.3. Outline of the proof

0.4. Further directions

0.5. Acknowledgements.

1. Basics 13

1.1. Looijenga pairs 13

1.2. Tropical Looijenga pairs 15

1.3. The Mumford degeneration and Givental's construction 19

2. Modified Mumford degenerations 23

2.1. The uncorrected degeneration 23

2.2. Scattering diagrams on $B \quad 28$

2.3. Broken lines $\quad 31$

2.4. The algebra structure $\quad 39$

3. The canonical scattering diagram $\quad 40$

3.1. Definition 40

3.2. Consistency: Overview of the proof 45

3.3. Consistency: Reduction to the Gross-Siebert locus 48

3.4. Step V: The proof of Theorem 3.25 and the connection with [GPS09 63

4. Smoothness: Around the Gross-Siebert locus 66

5. The relative torus 72 
6. Extending the family over boundary strata

6.1. Theorem 0.2 in the case that $(Y, D)$ has a toric model

6.2. Proof of Theorems 0.1 and 0.2 in general

6.3. The case that $(Y, D)$ is positive

7. Looijenga's conjecture

7.1. Duality of cusp singularities

7.2. Cusp family

7.3. Thickening of the cusp family

7.4. Smoothness

\begin{tabular}{|l|}
\hline 74 \\
\hline 74 \\
\hline 78 \\
\hline 81 \\
\hline 84 \\
\hline 84 \\
\hline 86 \\
\hline 92 \\
\hline 102 \\
\hline 105 \\
\hline
\end{tabular}

References

\section{INTRODUCTION}

0.1. The main theorems. Throughout the paper $(Y, D)$ with $D=D_{1}+\cdots+D_{n}$ will denote a smooth rational projective surface over an algebraically closed field $\mathbb{k}$ of characteristic zero, with $D \in\left|-K_{Y}\right|$ a singular nodal curve. The divisor $D$ is necessarily either an irreducible rational nodal curve, or a cycle of $n \geq 2$ smooth rational curves. We call $(Y, D)$ a Looijenga pair for, as far as we know, their rich geometry was first investigated in [L81]. We cyclically order the components of $D$ and take indices modulo $n$. By assumption there is a holomorphic symplectic 2-form $\Omega$, unique up to scaling, on $Y \backslash D$, with simple poles along $D$, and thus $U:=Y \backslash D$ is a log Calabi-Yau surface.

Our main result is a canonical synthetic construction of the mirror family to such a pair. The construction gives an embedded smoothing of the $n$-vertex $\mathbb{V}_{n} \subset \mathbb{A}^{n}$, defined as, for $n \geq 3$, the $n$-cycle of coordinate planes in $\mathbb{A}^{n}$ :

$$
\mathbb{V}_{n}:=\mathbb{A}_{x_{1}, x_{2}}^{2} \cup \mathbb{A}_{x_{2}, x_{3}}^{2} \cup \cdots \cup \mathbb{A}_{x_{n}, x_{1}}^{2} \subset \mathbb{A}_{x_{1}, \ldots, x_{n}}^{n} .
$$

(See (1.7) and (1.8) for the definition of $\mathbb{V}_{1}$ and $\mathbb{V}_{2}$.) This family is in general parameterized roughly by the formal completion of the affine toric variety $\operatorname{Spec} \mathbb{k}[\mathrm{NE}(Y)]$ along the union of toric boundary strata corresponding to contractions $f: Y \rightarrow \bar{Y}$. Here $\mathrm{NE}(Y)$ denotes the monoid $\mathrm{NE}(Y)_{\mathbb{R}} \cap A_{1}(Y, \mathbb{Z})$ where $\mathrm{NE}(Y)_{\mathbb{R}} \subset A_{1}(Y, \mathbb{R})$ is the cone generated by effective curve classes. This is just an approximate statement of our result, as $\mathrm{NE}(Y)$ is not in general finitely generated.

More precisely, fix $(Y, D), D=D_{1}+\cdots+D_{n}$ as above. Let $B_{0}(\mathbb{Z})$ be the set of pairs $(E, n)$ where $E$ is a prime divisor on some blowup of $Y$ along which $\Omega$ has a pole and $n$ is a positive integer. Set $B(\mathbb{Z}):=B_{0}(\mathbb{Z}) \cup\{0\}$. Later we will describe this set as the set of integer points in a natural integral affine manifold, the dual intersection 
complex, or tropicalization, of the pair $(Y, D)$. Let $v_{i} \in B(\mathbb{Z})$ be the pair $\left(D_{i}, 1\right)$. Choose $\sigma_{P} \subset A_{1}(Y, \mathbb{R})$ a strictly convex rational polyhedral cone containing $\mathrm{NE}(Y)_{\mathbb{R}}$, let $P:=\sigma_{P} \cap A_{1}(Y, \mathbb{Z})$ be the associated monoid, and set $R:=\mathbb{k}[P]$ to be the associated $\mathbb{k}$-algebra.

For each monomial ideal $I \subset R$, consider the free $R_{I}:=R / I$-module

$$
A_{I}:=\bigoplus_{q \in B(\mathbb{Z})} R_{I} \cdot \vartheta_{q} .
$$

Let $\mathfrak{m} \subset R$ denote the maximal monomial ideal. Let $T^{D}:=\mathbb{G}_{m}^{n}$ be the torus with character group $\chi\left(T^{D}\right)$ having basis $e_{D_{i}}$ indexed by the components $D_{i} \subset D$. There is a homomorphism $T^{D} \rightarrow \operatorname{Spec} \mathbb{k}\left[P^{\text {gp }}\right]$ induced by $C \mapsto \sum\left(C \cdot D_{i}\right) e_{D_{i}}$, so $T^{D}$ acts on $\operatorname{Spec} R_{I}$.

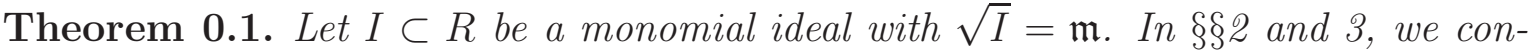
struct a finitely generated $R_{I}$-algebra structure on $A_{I}$, determined by relative GromovWitten invariants of $(Y, D)$ counting rational curves meeting $D$ in a single point. In \$5, we construct a $T^{D}$ action on Spec $A_{I}$. This induces a flat $T^{D}$-equivariant map

$$
f: X_{I}:=\operatorname{Spec} A_{I} \rightarrow \operatorname{Spec} R_{I}
$$

with closed fibre $\mathbb{V}_{n}$. By taking the limit over all such I, this yields a formal flat family

$$
\mathfrak{f}: \mathfrak{X}_{\mathfrak{m}} \rightarrow \mathfrak{S}_{\mathfrak{m}}:=\operatorname{Spf} \widehat{R},
$$

where $\widehat{R}$ is the completion of $R$ with respect to the ideal $\mathfrak{m}$. The generic fibre of $f$ is smooth in the sense of Definition 4.2. so $\mathfrak{f}$ is a formal smoothing of $\mathbb{V}_{n}$.

We use the notation $\vartheta_{q}$ for generators of our algebra, as the construction fits into a more general family of constructions which includes, as a special case, theta functions on abelian varieties. The history of such functions is as follows. Tyurin conjectured the existence of canonical theta functions (i.e., a basis of global sections) for polarized K3 surfaces, see Ty99. In 2007, discussions of the first author with Abouzaid and Siebert involving a tropicalization of the Fukaya category gave a stronger hint as to the existence of theta functions on arbitrary degenerations of Calabi-Yau manifolds in the context of the Gross-Siebert program. In particular, these discussions led to what is now understood to be a variant of the notion of broken line.

The latter notion was introduced in G09]. These were initially used to construct canonical perturbations of the Landau-Ginzburg potential for $\mathbb{P}^{2}$. Broken lines were then used for constructing mirror Landau-Ginzburg potentials for varieties with effective anti-canonical divisor in the setting of the Gross-Siebert program by Carl, Pumperla and Siebert in [CPS]. The authors show that the mirrors to such varieties as constructed in GS07] carry a canonical Landau-Ginzburg potential obtained by using 
broken lines to lift monomial functions on the central fibre of a toric degeneration to the toric degeneration.

Simultaneously, we used these same lifts to allow an extension of the construction developed by Gross and Siebert to prove the above main theorem. The main innovations we have introduced here are that we use theta functions to provide partial compactifications of certain canonically constructed deformations, and that these canonically constructed deformations, along with the theta functions, can be constructed relying only on the Gromov-Witten theory of $(Y, D)$. The key point is that it is easy to build deformations of the punctured $n$-vertex $\mathbb{V}_{n}^{o}:=\mathbb{V}_{n} \backslash\{0\}$, but it is difficult to extend these to deformations of $\mathbb{V}_{n}$. This is effectively done by using theta functions to embed a suitably chosen deformation of $\mathbb{V}_{n}^{o}$ in affine space, where the closure may then be taken. This extension would be impossible without the existence of theta functions.

This result can be viewed as log analogs of Tyurin's conjecture. In work in progress we apply similar ideas to obtain Tyurin's conjecture in the K3 case as well, and construct canonical bases for cluster algebras, to cite two other generalizations. These are large topics and will be expanded on elsewhere. See also GSTheta for more motivation from mirror symmetry, and upcoming papers [GHKS] and [K3].

Continuing with $(Y, D), P$ and $R$ as above, our second main theorem is:

Theorem 0.2. There is a unique smallest radical monomial ideal $J \subset R$ with the following properties:

(1) For every monomial ideal $I$ with $J \subset \sqrt{I}$ there is a finitely generated $R_{I^{-}}$

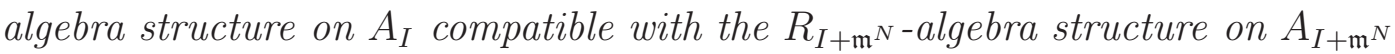
of Theorem 0.1 for all $N>0$.

(2) If the intersection matrix $\left(D_{i} \cdot D_{j}\right)$ is not negative semi-definite then $J=0$. In general, the zero locus $V(J) \subset$ Spec $R$ contains the union of the closed toric strata corresponding to faces $F$ of $\sigma_{P}$ such that there exists an $i$ such that $\left[D_{i}\right] \notin F$.

(3) Let $\widehat{R}$ denote the J-adic completion of $R$ and $\mathfrak{S}_{J}:=\operatorname{Spf} \widehat{R}$ the associated formal scheme. The algebras $A_{I}$ determine a canonical $T^{D}$-equivariant formal flat family of affine surfaces

$$
\mathfrak{f}: \mathfrak{X}_{J} \rightarrow \mathfrak{S}_{J}
$$

with fibre $\mathbb{V}_{n}$ over 0 . The $\vartheta_{q}$ determine a canonical embedding $\mathfrak{X}_{J} \subset \mathbb{A}^{\max (n, 3)} \times$ $\mathfrak{S}_{J}$.

Remark 0.3. When $\mathrm{NE}(Y) \subset P^{\prime} \subset P \subset A_{1}(Y)$, then $J^{\prime} \subset J$ and the formal family $\mathcal{X}$ for $P$ comes from the family for $P^{\prime}$ by base-change. In this sense the family is independent of the choice of $P$. 
Remark 0.4. Note that in the case that the intersection matrix $\left(D_{i} \cdot D_{j}\right)$ is not negative semi-definite (which includes the case that $D$ supports an ample divisor), Theorem 0.2 tells us that our construction gives a family over $\operatorname{Spec} R$, so in particular the construction is algebraic.

In this paper, we will not address the question as to in what sense our construction can be proved to be a mirror family. We expect, however, that our families constructed by the above theorems are mirror to $U=Y \backslash D$ in the sense of homological mirror symmetry in the case $\mathbb{k}=\mathbb{C}$. Further justification for our construction yielding the mirror family comes from the heuristic description of the construction in terms of symplectic geometry as discussed below.

The third main result of this paper is an application of our general construction, following from a more detailed analysis of the case where the matrix $\left(D_{i} \cdot D_{j}\right)$ is negative definite:

Theorem 0.5 (Looijenga's conjecture). A 2-dimensional cusp singularity is smoothable if and only if the exceptional cycle of the dual cusp occurs as an anti-canonical cycle on a smooth projective rational surface.

This was conjectured by Looijenga in [L81, where he also proved the forward implication. Partial results were obtained in [FM83] and [FP84].

0.2. The symplectic heuristic. Much of what we do in this paper, following the philosophy of the Gross-Siebert program, is to tropicalize the SYZ picture [SYZ96]. Thus it is helpful to review informally this picture in the context of mirrors to Looijenga pairs $(Y, D)$. The SYZ picture will be a heuristic philosophical guide, and hence we make no effort to be rigorous. Here we follow the exposition from [A07] concerning SYZ on the complement of an anti-canonical divisor, itself a generalization of ideas of Cho and $\mathrm{Oh}$ for interpreting the Landau-Ginzburg mirror of a toric variety in terms of counting Maslov index two holomorphic disks [CO06]. For the most part we follow Auroux's notation, except that we use $Y$ instead of his $X$, and our $X$ is his $M$.

We fix a Kähler form $\omega$ on $Y$, and a nowhere vanishing holomorphic 2-form $\Omega$ on $U:=Y \backslash D$. Now suppose we have a fibration $f: U \rightarrow B$ by special Lagrangian 2-tori (i.e., a fibre $L$ of $f$ satisfies $\left.\operatorname{Im} \Omega\right|_{L}=\left.\omega\right|_{L}=0$ ). Then the SYZ mirror $X$ of $(U, \omega)$ is the dual torus fibration $\check{f}: X \rightarrow B$. This can be thought of as a moduli space of pairs $(L, \nabla)$ consisting of a special Lagrangian fibre $L$ of $f$ equipped with a unitary connection $\nabla$ modulo gauge equivalence, or equivalently a holonomy map $\operatorname{hol}_{\nabla}: H_{1}(L, \mathbb{Z}) \rightarrow U(1) \subset \mathbb{C}^{*}$. The complex structure on $X$ is subtle, specified by so-called instanton corrections. 
In this picture we can define local holomorphic functions on $X$ associated to a basis of $H_{2}(Y, L, \mathbb{Z}$ ) (in a neighbourhood of a fibre of $\check{f}$ corresponding to a non-singular fibre $L$ of $f)$ as follows. For $A \in H_{2}(Y, L, \mathbb{Z})$ define

$$
z^{A}:=\exp \left(-2 \pi \int_{A} \omega\right) \operatorname{hol}_{\nabla}(\partial A): X \rightarrow \mathbb{C}^{*} .
$$

By choosing a splitting of $H_{2}(Y, L, \mathbb{Z}) \rightarrow H_{1}(L, \mathbb{Z})$ we can pick out local coordinates on $X$ which define a complex structure. See [A07, Lemma 2.7. Note that as the fibre $L$ varies, the relative homology group $H_{2}(Y, L, \mathbb{Z})$ forms a local system over $B_{0} \subset$ $B$, where $B_{0}$ is the subset of points with non-singular fibres. This local system has monodromy, and as a consequence, the functions $z^{A}$ are only well-defined locally.

However, there are also well-defined global functions $\vartheta_{1}, \ldots, \vartheta_{n}$ on $X$. These are defined locally in neighbourhoods of fibres of $\check{f}$ corresponding to fibres of $f$ not bounding holomorphic disks contained in $U$, via a (rough) expression

$$
\vartheta_{i}=\sum_{\beta \in H_{2}(Y, L, \mathbb{Z})} n_{\beta} z^{\beta}
$$

where $n_{\beta}$ is a count of so-called Maslov index two disks with boundary on $L$ representing the class $\beta$ and intersecting $D$ transversally in one point lying in $D_{i}$. (We note that in our setting the Maslov index $\mu$ of a holomorphic disk $f: \Delta \rightarrow Y$ with boundary lying on a special Lagrangian torus $L \subset Y$ is given by $\mu=2 \operatorname{deg} f^{*} D$. See [A07, Lemma 3.1.) In the case that $D$ is ample, there are, for generic $L$, only finitely many such disks; it is not known how to treat the general case in this symplectic setting.

For $\vartheta_{i}$ to make sense the moduli space of Maslov index 2 disks with boundary on $L$ must deform smoothly with the Lagrangian $L$. This fails for Lagrangians that bound holomorphic disks contained in $U$ (Maslov index zero disks). This is a real codimension one condition on $L$, and thus defines canonical walls in the affine manifold $B$. When we cross the wall the $\vartheta_{i}$ are discontinuous. But the discontinuity is corrected by a holomorphic change of variable in the local coordinates $z^{\beta}$, according to [A07, Proposition 3.9:

$$
z^{\beta} \rightarrow z^{\beta} \cdot h\left(z^{\alpha}\right)^{[\partial \beta] \cdot[\partial \alpha]}
$$

where here $\alpha \in H_{2}\left(Y, L_{0}, \mathbb{Z}\right)$ represents the class of the Maslov index zero disk with boundary on $L_{0}$ a Lagrangian fibre over a point on the wall, and $h(q)$ is a generating function counting such holomorphic disks. Thus we can define a new complex manifold, with the same local coordinates, by composing the obvious gluing induced by identifications of fibres of the local system on $B_{0}$ with fibres $H_{2}(Y, L, \mathbb{Z})$ with the automorphism (0.4). These regluings are the instanton corrections, and the modified manifold $X$ should be the mirror. By construction it comes with canonical global 
holomorphic functions $\vartheta_{i}$. In particular, the sum $W=\sum_{i} \vartheta_{i}$ is a well-defined global function, the Landau-Ginzburg potential.

0.3. Outline of the proof. We now outline how we realise the symplectic SYZ heuristic in terms of algebraic geometry. There are three principal issues to consider:

- What information about a putative SYZ fibration can be seen inside algebraic geometry?

- What is the analogue of a Maslov index two disk in algebraic geometry?

- How do we obtain the mirror by gluing together varieties?

The philosophy for dealing with the first and third issues was developed by Gross and Siebert in GS07. For the first item, while we cannot build an SYZ fibration $f: U \rightarrow B$ in general, we can roughly describe $B$ as a combinatorial object. Given the Looijenga pair $(Y, D)$, we build a space $B$ homeomorphic to $\mathbb{R}^{2}$ along with a decomposition $\Sigma$ of $B$ into cones. We construct $(B, \Sigma)$ as the dual intersection complex of $(Y, D)$. For each double point of $D$, we take a copy of the first quadrant in $\mathbb{R}^{2}$, with the axes labelled by the two irreducible components of $D$ (assuming $D$ is not irreducible) passing through the double point. We then identify edges of these cones if they are labelled with the same irreducible component of $D$. We thus get a topological space abstractly homeomorphic to $\mathbb{R}^{2}$ subdivided into cones. This is $(B, \Sigma)$. In $\$ 1.2$, we show how we can put an additional structure on $B$, namely the structure of an affine manifold with singularities. Indeed, we can give $B_{0}:=B \backslash\{0\}$ a system of coordinate charts whose transition maps are integral affine linear transformations. The affine structure does not extend across the origin unless $(Y, D)$ is in fact a toric pair, in which case we recover the fan $\Sigma$ defining $Y$.

The manifold $B$ can be viewed as the base of the SYZ fibration "seen from a great distance." In general the base of an SYZ fibration has the structure of an affine manifold with singularities. Singular fibres of the fibration occur over the singular points. One would expect $f: U \rightarrow B$ to have a number of singular fibres in general, hence $B$ will have a number of singular points. So the above construction moves all these singular points to the origin.

Next, let us consider the third item. Fixing $(Y, D)$ with $D=D_{1}+\cdots+D_{n}$, let $P \subset A_{1}(Y, \mathbb{Z})$ be a finitely generated monoid containing the classes of all effective curves on $Y$, obtained by choosing a strictly convex rational polyhedral cone $\sigma_{P} \subset A_{1}(Y, \mathbb{R})$ containing the Mori cone. Let $\mathfrak{m}$ be the maximal monomial ideal in the ring $\mathbb{k}[P], I$ a monomial ideal with radical $\mathfrak{m}$, and let $R_{I}=\mathbb{k}[P] / I$.

We will describe the basic pieces we will glue together to describe a scheme over $S_{I}:=\operatorname{Spec} R_{I}$ whose special fibre is $\mathbb{V}_{n}^{o}:=\mathbb{V}_{n} \backslash\{0\}$. Assume that the components $D_{i}$ 
are numbered in cyclic order, with indices taken modulo $n$. We can define an open cover of $\mathbb{V}_{n}^{o}$ by taking sets, for $1 \leq i \leq n$,

$$
U_{i}=V\left(X_{i-1} X_{i+1}\right) \subset \mathbb{A}_{X_{i-1}, X_{i+1}}^{2} \times\left(\mathbb{G}_{m}\right)_{X_{i}}
$$

Note as subsets of $\mathbb{V}_{n}$, they are disjoint except for

$$
U_{i, i+1}:=U_{i} \cap U_{i+1}=\left(\mathbb{G}_{m}\right)_{X_{i}, X_{i+1}}^{2} .
$$

In $\mathbb{V}_{n}$ they are glued in the obvious way, i.e., via the canonical inclusions

$$
U_{i, i+1}=\left\{X_{i+1} \neq 0\right\} \subset U_{i}, \quad U_{i, i+1}=\left\{X_{i} \neq 0\right\} \subset U_{i+1} .
$$

A deformation of $\mathbb{V}_{n}^{o}$ over $S_{I}$ is obtained by gluing thickenings of the $U_{i}$

$$
U_{i, I}:=V\left(X_{i-1} X_{i+1}-z^{\left[D_{i}\right]} X_{i}^{-D_{i}^{2}}\right) \subset S_{I} \times \mathbb{A}_{X_{i-1}, X_{i+1}}^{2} \times\left(\mathbb{G}_{m}\right)_{X_{i}}
$$

where $z^{\left[D_{i}\right]} \in \mathbb{k}[P]$ is the corresponding monomial. The overlaps are relative tori, $U_{i, i+1, I}=S_{I} \times \mathbb{G}_{m}^{2}$, and the gluings are the obvious ones. The details are given in $\$ 2.1$. This gluing gives a flat family $X_{I}^{o} \rightarrow S_{I}$, which can be viewed as being analogous to the naive complex structure on the mirror described as the moduli of smooth special Lagrangian fibres with $U(1)$ connection.

There is no reason in general to believe that $X_{I}^{o} \rightarrow S_{I}$ can be extended to a flat deformation $X_{I} \rightarrow S_{I}$ of $\mathbb{V}_{n}$. The reason is that such an $X_{I}$ should be an affine scheme, and hence have many functions, while $X_{I}^{o}$ as constructed tends to have few functions. The only case where $X_{I}^{o}$ extends to give a deformation of $\mathbb{V}_{n}$ is when $(Y, D)$ is a toric pair. In this case, we recover an infinitesimal version of Givental's mirror family, which then easily extends to Givental's mirror construction. We review this case in $\$ 1.3$,

To rectify this problem, we need to translate the instanton corrections of the symplectic heuristic. We do this using the notion of scattering diagram, here a variant of similar notions introduced in [KS06] and [GS07.

For us, a scattering diagram $\mathfrak{D}$ will be a collection of pairs $\left(\mathfrak{d}, f_{\mathfrak{d}}\right)$ where $\mathfrak{d}$ is a ray emanating from the origin of $B$ with rational slope, and $f_{\mathfrak{d}}$ is a kind of function attached to the ray. Any scattering diagram will dictate how to modify both the definition of the open sets $U_{i, I}$ and the gluings of $U_{i, I}$ with $U_{i+1, I}$. The precise details of this modification are given in $\$ 2.2$. Briefly, the rays define automorphisms of the open sets $U_{i, i+1, I}$ analogous to (0.4), and are used to modify the gluing.

While any scattering diagram can be used to obtain a modified flat deformation $X_{I, \mathfrak{D}}^{o}$, we need to choose $\mathfrak{D}$ correctly to have a chance of extending this deformation to $\mathbb{V}_{n}$. The symplectic heuristic can be used to motivate the choice of the canonical scattering diagram. The functions $f_{\mathfrak{d}}$ chosen are generating functions for certain Gromov-Witten 
invariants, intuitively counting finite maps $\mathbb{A}^{1} \rightarrow U$. Heuristically, each holomorphic disk contributing can be approximated by a proper rational curve meeting $D$ in a single point.

Thus the canonical scattering diagram encodes the chamber structure seen in the symplectic heuristic. But there still remains the question of extending $X_{I, \mathfrak{D}}^{o}$ to a flat deformation of $\mathbb{V}_{n}$. To do so, we need to construct enough functions on $X_{I, \mathfrak{D}}^{o}$. This is where the concept of theta function comes in. The symplectic heuristic suggests that there should be a canonical choice of holomorphic functions on $X_{I, \mathfrak{D}}^{o}$ arising from a count of Maslov index two holomorphic disks. Rather than trying to find an algebro-geometric analogue of a Maslov index two holomorphic disk, one instead defines the counts using tropical geometry. In particular, we use the notion of broken line, introduced in G09] and developed further by [CPS] simultaneously with this work, to provide the count. A broken line is essentially a tropical analogue of a Maslov index two disk. They are piecewise linear paths which only bend when they cross rays of the scattering diagram $\mathfrak{D}$, in ways prescribed by the functions attached to the rays.

For any point $p \in B$ with integral coordinates, we can use a count of broken lines to define a function on $U_{i, I}$ for any $i$. This procedure is described in $\$ 2.3$, Since this procedure is dependent on the scattering diagram $\mathfrak{D}$, we can then ask whether these functions on the various $U_{i, I}$ glue. We say $\mathfrak{D}$ is consistent if they always glue. If these functions do glue, then we call the resulting global function on $X_{I, \mathfrak{D}}^{o}$ a theta function, writing it as $\vartheta_{p}$.

The bulk of the argument in this paper occurs in \$3. where we prove that the canonical scattering diagram described above is in fact consistent. This argument is rather involved, so we leave it to \$3.2 to give an overview of the full argument for consistency. Crucial to the argument is a reduction to methods of [CPS] using the main results of GPS09].

Once consistency is proved, this gives global functions $\vartheta_{p}$ on $X_{I, \mathfrak{D}}^{o}$ for each $p \in B$ with integral coordinates. Let $v_{i}$ denote the first integral point along the ray of $\Sigma$ corresponding to the divisor $D_{i}$, and write $\vartheta_{i}:=\vartheta_{v_{i}}$. Then we can use the functions $\vartheta_{1}, \ldots, \vartheta_{n}$ to embed (in the case that $n \geq 3$ ) $X_{I, \mathfrak{D}}^{o}$ in $\mathbb{A}^{n} \times S_{I}$. Taking the closure of the image gives the desired deformation $X_{I} \rightarrow S_{I}$ of $\mathbb{V}_{n}$.

This construction essentially proves the first main theorem, Theorem 0.1 . The statement about the scheme-theoretic singular locus of $f$ is dealt with in $\$ 4$. There we again make a connection with the techniques of [GS07. The crucial point is to show the singularity $0 \in \mathbb{V}^{n}$ is formally smoothed, and for this, we need to work in a family where we have a local model for the behaviour near 0, much as Gross and Siebert have in GS07]. 
More work is required for Theorem 0.2. We need to show that the construction above, which really only produces a family over the completion of $\operatorname{Spec} \mathbb{k}[P]$ at the zero-dimensional torus orbit of this scheme, extends across completions along larger strata. Since the coordinate rings of the families constructed above are generated by theta functions, we proceed by studying the products of theta functions. In general, one expects the product of two theta functions to be a formal series of theta functions. However, in many cases one can control the terms sufficiently in these products to obtain the desired extensions. This relies on a tropical interpretation of the product of theta functions, given in $\$ 2.4$, as well as the existence of a torus action on our families, given in \$5. This torus action only exists because of the canonical nature of our scattering diagrams. Complete details for the arguments are given in the last section, \$6.

Turning to Theorem 0.5, the main point is that Looijenga's conjecture is really a form of mirror symmetry. We start with a pair $(Y, D)$ such that the intersection matrix $\left(D_{i} \cdot D_{j}\right)$ is negative definite. Thus $D$ can be contracted analytically to give a cusp singularity $p \in Y^{\prime}$. (By definition, a cusp singularity is a surface singularity whose minimal resolution is a cycle of rational curves.) For the sake of exposition, assume this contraction is algebraic, so that there is a divisor $L$ on $Y$ which is the pull-back of an ample divisor on $Y^{\prime}$. We choose the monoid $P$ so $L^{\perp} \cap P$ is a face $P_{\text {bdy }}$ of $P$, with $P_{\text {bdy }}^{\mathrm{gp}}$ generated by the classes $\left[D_{1}\right], \ldots,\left[D_{n}\right]$. The main goal is to extend our construction to a formal neighbourhood of $\operatorname{Spec} \mathbb{k}\left[P_{\text {bdy }}\right] \subset \operatorname{Spec} \mathbb{k}[P]$. The problem is that Theorem 0.2 explicitly does not apply in this case. The main difficulty is that the charts (0.5) overlap too much when all the $z^{\left[D_{i}\right]}$ are invertible (in fact the fibres over such points in $\operatorname{Spec} \mathbb{k}\left[P_{\text {bdy }}\right]$ coincide under the natural gluing maps). There is no way to glue these charts compatibly. However, this can be done after shrinking these charts to analytic open subsets and working over an analytic open neighborhood of the zero-dimensional stratum of $\operatorname{Spec} \mathbb{k}\left[P_{\text {bdy }}\right]$. Here we work of course with $\mathbb{k}=\mathbb{C}$ only.

In doing so, we find over a general point of $\operatorname{Spec} \mathbb{k}\left[P_{\mathrm{bdy}}\right]$ the dual cusp singularity to $p \in Y^{\prime}$. Thus we see that our mirror symmetry construction naturally produces the dual cusp. We then would like to extend the family constructed over thickenings of Spec $\mathbb{k}\left[P_{\mathrm{bdy}}\right]$. We use the same techniques as those used to prove Theorem 0.1 . However, the construction of theta functions is considerably more delicate. In general, theta functions are described as a sum of monomials associated to broken lines. In the situation of Theorem 0.1 , these sums are always finite. However, in the current situation, they are always infinite. Thus there are serious convergence issues, and this makes the proof rather technical. A delicate analysis of the combinatorics of broken lines is necessary to prove convergence. 
Once convergence is shown, we then argue that the formal family produced actually gives a smoothing of the cusp singularity. This follows from the fact proved in Theorem 0.1 that we already have a smoothing of the $n$-vertex in a formal neighbourhood of the zero-dimensional stratum, but again the argument is slightly delicate. All details are given in $\$ 7$.

0.4. Further directions. Here we will briefly indicate the results of further study of our mirror construction, to be given in sequel papers, as well as connections with other recent work.

There are three broad classes of behaviour for our construction, depending on the properties of the intersection matrix $\left(D_{i} \cdot D_{j}\right)$ : the matrix can be negative definite, negative semi-definite but not negative definite, or not negative semi-definite. The first case is analyzed here in detail to prove Theorem 0.5. We will discuss the third case in the sequel paper.

We call the case that the intersection matrix is not negative semi-definite the positive case. It holds if and only if $U$ is the minimal resolution of an affine surface, see Lemma 6.9. In this case, the cone $\mathrm{NE}(Y)_{\mathbb{R}}$ is rational polyhedral, so we may take $P=\mathrm{NE}(Y)$. Furthermore, the ideal $J$ of Theorem 0.2 equals 0 . Thus our construction defines an algebraic family over $\operatorname{Spec} \mathbb{k}[\mathrm{NE}(Y)]$, with smooth generic fibre. We will show in Part II that the restriction of this family to the structure torus

$$
\mathcal{X} \rightarrow T_{Y}:=\operatorname{Pic}(Y) \otimes \mathbb{G}_{m}=\operatorname{Spec} \mathbb{k}\left[A_{1}(Y)\right] \subset \operatorname{Spec} \mathbb{k}[\mathrm{NE}(Y)]
$$

is close to a universal family of deformations of $U=Y \backslash D$.

More precisely, we will show independently of the positivity of the intersection matrix that our formal family has a simple and canonical (fibrewise) compactification to a formal family $(\mathcal{Z}, \mathcal{D})$ of Looijenga pairs (with $\mathcal{X}=\mathcal{Z} \backslash \mathcal{D}$ ), equivariant for the action of $T^{D} \subset T_{Y}$, the subtorus generated by the components of $D$. The theta functions are $T^{D}$ eigenfunctions, see $\$ 5$.

In the positive case this extends naturally over all of $\operatorname{Spec} \mathbb{k}[\mathrm{NE}(Y)]$, and its restriction $(\mathcal{Z}, \mathcal{D}) \rightarrow T_{Y}$ comes with a trivialization of the boundary $\mathcal{D}=D_{*} \times T_{Y}$ realizing it as the universal family of Looijenga pairs $\left(Z, D_{Z}\right)$ deformation equivalent to $(Y, D)$ together with an isomorphism $D_{Z} \stackrel{\sim}{\rightarrow} D_{*}$ constructed in [GHK12]. In particular, choosing such an isomorphism $D \stackrel{\sim}{\rightarrow} D_{*}$ for our original pair $(Y, D)$ canonically identifies it with a fibre of the family $(\mathcal{Z}, \mathcal{D}) / T_{Y}$. More importantly, the restrictions of the theta functions $\vartheta_{q}$ to $U \subset \mathcal{X}$ endow the affine surface $U=Y \backslash D$ with canonical functions. We give a modular interpretation of the quotient of $\mathcal{Z} \backslash \mathcal{D} \rightarrow T_{Y}$ by $T^{D}$ as the universal deformation of $U$ (this shows in particular the quotient depends only on $U$, e.g., 
is independent of the choice of compactification $U \subset Y$ ), and give a unique geometric characterisation of the theta function basis of $H^{0}\left(U, \mathcal{O}_{U}\right)$.

The fact that $(Y, D)$ appears as a fibre is perhaps a bit surprising as, after all, we set out to construct the mirror and have obtained the original surface back. Note however that dual Lagrangian torus fibrations in dimension 2 are topologically equivalent by Poincaré duality, so this is consistent with the SYZ formulation of mirror symmetry.

To illustrate, in Example 6.12 we explicitly compute the theta functions in the case $(Y, D)$ is the del Pezzo of degree 5 together with a cycle of $5(-1)$-curves. In Example 6.13. we give the expression in the case of a triangle of lines on a cubic surface, deferring in this case the proof until Part II. In each of these cases there is a characterisation of the $\vartheta_{v_{i}}$ in terms of classical geometry.

In a different direction, in GHKK, along with M. Kontsevich, we extend many of the methods introduced in this paper to prove a number of significant conjectures about cluster varieties. In particular, the technology of theta functions leads to a proof of positivity of the Laurent phenomenon, and a proof of the Fock-Goncharov dual basis conjecture for a broad class of cluster varieties. The latter can be viewed as a generalization of the construction of theta functions on $Y \backslash D$ in the positive case, described above. In fact, in the case of cluster varieties associated to a skew-symmetric matrix of rank two, the Fock-Goncharov $\mathcal{X}$ variety fibres over a torus with fibres being interiors of Looijenga pairs. This is described in detail in GHK13. In this case, the general construction of theta functions in [GHKK] coincides with the ones constructed here.

Let us end with some mild speculation in all dimensions suggested by the above discussion. By a Looijenga pair we mean a dlt pair $(Y, D)$ (e.g., a simple normal crossings pair) with $K_{Y}+D$ trivial and $(Y, D)$ having a zero-dimensional log canonical center. (In the simple normal crossing case, this means there is an intersection point of $\operatorname{dim} Y$ different components of D.) By a $\log$ Calabi-Yau with maximal boundary we mean a variety $U$ which can be realized as the interior $Y \backslash D$ of a Looijenga pair. See $\S 1$ of GHK13] for background on these notions. We expect that many of the results in this paper will extend to Looijenga pairs of all dimensions. This generalization will require the further development of the technology of logarithmic Gromow-Witten invariants, GS11], AC11]. We should obtain in complete generality a mirror family $\mathcal{X} \rightarrow$ Spec $\widehat{\mathbb{k}[P]}$ for suitable monoids $P$. Furthermore, one would expect in the case that $U=Y \backslash D$ is affine that this family extends to $\mathcal{X} \rightarrow \operatorname{Spec} \mathbb{k}[P]$. Using the twodimensional case as a guide, the general fibre of $\mathcal{X} \rightarrow \operatorname{Spec} \mathbb{k}[P]$ should itself be the interior of a Looijenga pair $(\bar{X}, E)$, with $\bar{X} \backslash E$ affine by construction. Thus we can then repeat the process to obtain a family $\mathcal{X}^{\prime} \rightarrow \operatorname{Spec} \mathbb{k}\left[P^{\prime}\right]$, and it would be expected, as 
taking mirror twice should return to where we started, that $\mathcal{X}^{\prime} \rightarrow \operatorname{Spec} \mathbb{k}\left[P^{\prime}\right]$ contains a fibre isomorphic to $U$. The family $\mathcal{X}^{\prime}$ carries our canonically defined theta functions, indexed by tropical points of the mirror. This leads us to propose:

Conjecture 0.6. Let $U$ be an affine log Calabi-Yau variety with maximal boundary. Then $H^{0}\left(U, \mathcal{O}_{U}\right)$ has a canonical basis of theta functions indexed by tropical points of the mirror. The structure constants for multiplication of theta functions can be described combinatorially in terms of broken lines.

Versions of this conjecture have been proven for cluster varieties in many cases in GHKK.

0.5. Acknowledgements. An initial (and ongoing) motivation for the project was to find a geometric compactification of moduli of polarized K3 surfaces. We received a good deal of initial inspiration in this direction from conversations with $\mathrm{V}$. Alexeev. The project also owes a great deal to the first author's collaboration with B. Siebert. We learned a great many things from A. Neitzke, especially about the connections of our work with cluster algebras and moduli of local systems. Our thinking about Looijenga pairs was heavily influenced by conversations with R. Friedman and E. Looijenga. Many other people have helped us with the project, and discussions with D. Allcock, D. Ben-Zvi, V. Fock, D. Freed, A. Goncharov, R. Heitmann, D. Huybrechts, M. Kontsevich, A. Oblomkov, T. Perutz, M. Reid, A. Ritter, and Y. Soibelman were particularly helpful. We would also like to thank IHÉS for hospitality during the summer of 2009 when part of this research was done. The first author was partially supported by NSF grants DMS-0805328 and DMS-0854987. The second author was partially supported by NSF grant DMS-0968824 and DMS-1201439. The third author was partially supported by NSF grant DMS-0854747.

\section{BASICS}

\subsection{Looijenga pairs.}

Definition 1.1. A Looijenga pair $(Y, D)$ is a smooth rational projective surface $Y$ together with a reduced nodal curve $D \in\left|-K_{Y}\right|$ with at least one singular point.

Note that for a Looijenga pair, $p_{a}(D)=1$ by adjunction. Since $H^{1}\left(Y, \mathcal{O}_{Y}\right)=0$ by rationality of $Y, D$ is connected. Applying adjunction to each irreducible component of $D$, one sees easily that $D$ is either an irreducible genus one curve with a single node, or a cycle of smooth rational curves. We will always write $D=D_{1}+\cdots+D_{n}$, with a cyclic ordering of the irreducible components, and take the indices modulo $n$.

We will need a few basic facts about Looijenga pairs, which we collect here. 
Definition 1.2. Let $(Y, D)$ be a Looijenga pair.

(1) A toric blow-up of $(Y, D)$ is a birational morphism $\pi: \tilde{Y} \rightarrow Y$ such that if $\tilde{D}$ is the reduced scheme structure on $\pi^{-1}(D)$, then $(\tilde{Y}, \tilde{D})$ is a Looijenga pair. In particular, $\tilde{Y}$ is smooth.

(2) A toric model of $(Y, D)$ is a birational morphism $(Y, D) \rightarrow(\bar{Y}, \bar{D})$ to a smooth toric surface $\bar{Y}$ with its toric boundary $\bar{D}$ such that $D \rightarrow \bar{D}$ is an isomorphism.

Note that if $\pi: \tilde{Y} \rightarrow Y$ is the blow-up of a node of $D$, then $\pi$ is a toric blow-up.

Proposition 1.3. Given $(Y, D)$ there exists a toric blowup $(\tilde{Y}, \tilde{D})$ which has a toric $\operatorname{model}(\tilde{Y}, \tilde{D}) \rightarrow(\bar{Y}, \bar{D})$.

Proof. First observe:

(1) Let $p: Y \rightarrow Y^{\prime}$ be the blowdown of a $(-1)$-curve not contained in $D$, and $D^{\prime}:=p_{*}(D) \subset Y^{\prime}$. If the proposition holds for $\left(Y^{\prime}, D^{\prime}\right)$ then it holds for $(Y, D)$.

(2) Let $Y^{\prime \prime} \rightarrow Y$ be the blowup at a node of $D$, and $D^{\prime \prime} \subset Y^{\prime \prime}$ the reduced inverse image of $D$. The proposition holds for $\left(Y^{\prime \prime}, D^{\prime \prime}\right)$ if and only if it holds for $(Y, D)$.

By using (1) and (2) repeatedly we may assume $Y$ is minimal, and thus is either a ruled surface or is $\mathbb{P}^{2}$. In the latter case, by blowing up a node of $D$ we reduce to the ruled case. So we have $q: Y \rightarrow \mathbb{P}^{1}$ a ruling. We next consider the number of components of $D$ which are fibres of $q$. There cannot be more than two such components, for otherwise $D$ cannot be a cycle. If there are precisely two such components, then $D$ necessarily has precisely four components, and it is then easy to check that $D$ is the toric boundary of $Y$, for a suitable choice of toric structure on $Y$. In this case the proposition obviously holds. Otherwise let $D^{\prime} \subset D$ be the union of components not contained in fibres. If $D^{\prime}$ has a node, then we can blowup the node, blowdown the strict transform of the fibre through the node, increasing the number of components of $D$ contained in fibres.

After carrying out this procedure for each node of $D^{\prime}$, we are then in one of two cases.

Case I. $D$ has two components contained in fibres, and then we are done.

Case II. $D$ consists of a fibre $f$ and a non-singular irreducible two-section $D^{\prime}$ of $q$. Note that since $D^{\prime}+f \sim-K_{Y}$ and $Y$ is isomorphic to the Hirzebruch surface $\mathbb{F}_{e}$ for some $e$, we can write $\operatorname{Pic} Y=\mathbb{Z} C_{0} \oplus \mathbb{Z} f$, with $C_{0}^{2}=-e$ and $-K_{Y}=2 C_{0}+(e+2) f$. Thus $D^{\prime} \sim 2 C_{0}+(e+1) f$ and $C_{0} \cdot D^{\prime}=-e+1$. Since $C_{0}$ is not contained in $D^{\prime}, e=0$ or 1 .

If $e=0$, then there is a second ruling $q^{\prime}: Y \rightarrow \mathbb{P}^{1}$, with $D^{\prime}$ and $f$ sections of this ruling. In this case, we follow the same procedure as above of blowing up nodes for this new ruling, arriving in Case I. 
If $e=1$, then $C_{0}$ is disjoint from $D^{\prime}$. Blowing down $C_{0}$, we obtain $\mathbb{P}^{2}$, and can then blowup one of the nodes of the image of $D^{\prime} \cup f$. Using this new ruled surface, we can again blowup a node and find ourselves back in Case I.

1.2. Tropical Looijenga pairs. We explain how to tropicalize a Looijenga pair, first recalling the following basic definition. Fix a lattice $M \cong \mathbb{Z}^{n}$. In what follows, we will always use the notation $M_{\mathbb{R}}=M \otimes_{\mathbb{Z}} \mathbb{R}, N=\operatorname{Hom}_{\mathbb{Z}}(M, \mathbb{Z})$ and $N_{\mathbb{R}}=N \otimes_{\mathbb{Z}} \mathbb{R}$. We denote by $\operatorname{Aff}(M)$ the group of affine linear transformations of the lattice $M$. Recall the following definitions from [GS06].

Definition 1.4. An integral affine manifold $B$ is a (real) manifold $B$ with an atlas of charts $\left\{\psi_{i}: U_{i} \rightarrow M_{\mathbb{R}}\right\}$ such that $\psi_{i} \circ \psi_{j}^{-1} \in \operatorname{Aff}(M)$ for all $i, j$.

An integral affine manifold with singularities $B$ is a (real) manifold $B$ with an open subset $B_{0} \subset B$ which carries the structure of an integral affine manifold, and such that $\Delta:=B \backslash B_{0}$, the singular locus of $B$, is a locally finite union of locally closed submanifolds of codimension at least two.

If $B$ is an integral affine manifold with singularities, there is a local system $\Lambda_{B}$ on $B_{0}$ consisting of flat integral vector fields: if $y_{1}, \ldots, y_{n}$ are local integral affine coordinates, then $\Lambda_{B}$ is locally given by linear combinations of the vector fields $\partial / \partial y_{1}, \ldots, \partial / \partial y_{n}$. If $B$ is clear from context, we drop the subscript $B$.

Similarly, $\check{\Lambda}_{B}$ is the dual local system, locally generated by $d y_{1}, \ldots, d y_{n}$.

We will be primarily interested in $\operatorname{dim} B=2$ in this paper, in which case $\Delta$ will consist, in all our examples, of a finite number of points. All integral affine manifolds we encounter will in fact be linear, in the sense that the coordinate transformations are in fact linear rather than just affine linear.

We associate to a Looijenga pair $(Y, D)$ a pair $(B, \Sigma)$, where $B$ is homeomorphic to $\mathbb{R}^{2}$ and has the structure of integral affine manifold with one singularity at the origin, and $\Sigma$ is a decomposition of $B$ into cones. We call $(B, \Sigma)$ the tropicalization of $(Y, D)$, and $\Sigma$ the fan of $(Y, D)$. The idea is that we pretend that $(Y, D)$ is toric and we try to build the associated fan. More precisely, the construction is as follows.

For each node $p_{i, i+1}:=D_{i} \cap D_{i+1}$ of $D$ we take a rank two lattice $M_{i, i+1}$ with basis $v_{i}, v_{i+1}$, and the cone $\sigma_{i, i+1} \subset M_{i, i+1} \otimes_{\mathbb{Z}} \mathbb{R}$ generated by $v_{i}$ and $v_{i+1}$. We then glue $\sigma_{i, i+1}$ to $\sigma_{i-1, i}$ along the rays $\rho_{i}:=\mathbb{R}_{\geq 0} v_{i}$ to obtain a piecewise-linear manifold $B$ homeomorphic to $\mathbb{R}^{2}$ and a decomposition

$$
\Sigma=\left\{\sigma_{i, i+1} \mid 1 \leq i \leq n\right\} \cup\left\{\rho_{i} \mid 1 \leq i \leq n\right\} \cup\{0\}
$$


We define an integral affine structure on $B \backslash\{0\}$ by defining charts $\psi_{i}: U_{i} \rightarrow M_{\mathbb{R}}$ (where $M=\mathbb{Z}^{2}$ ). Here

$$
U_{i}=\operatorname{Int}\left(\sigma_{i-1, i} \cup \sigma_{i, i+1}\right)
$$

and $\psi_{i}$ is defined on the closure of $U_{i}$ by

$$
\psi_{i}\left(v_{i-1}\right)=(1,0), \quad \psi_{i}\left(v_{i}\right)=(0,1), \quad \psi_{i}\left(v_{i+1}\right)=\left(-1,-D_{i}^{2}\right),
$$

with $\psi_{i}$ linear on $\sigma_{i-1, i}$ and $\sigma_{i, i+1}$. The reason for choosing these particular vectors is that they form the one-dimensional rays of a fan defining a toric variety such that the divisor $D_{i}$ corresponding to the ray generated by $v_{i}$ has self-intersection $D_{i}^{2}$.

We note this construction makes sense even when $n=1$, i.e., the anti-canonical divisor $D$ is an irreducible nodal curve. In this case there is one cone $\sigma_{1,1}$, and opposite sides of the cone are identified. (Moreover, the integral affine charts are defined using the integer $D_{1}^{2}-2$ instead of $D_{1}^{2}$. This is the degree of the normal bundle of the map from the normalization of $D_{1}$ to $Y$.) However, this case will often complicate arguments in this paper, so we will usually replace $Y$ with a surface obtained by blowing up the node of $D$, and replace $D$ with the reduced inverse image of $D$ under the blowup. This does not change the underlying integral affine manifold with singularities, but refines the decomposition $\Sigma$, exactly as in the toric case:

Definition 1.5. Given $(B, \Sigma)$, a refinement is a pair $(B, \tilde{\Sigma})$, where $\tilde{\Sigma}$ is a decomposition of $B$ into rational polyhedral cones refining $\Sigma$, each cone of $\tilde{\Sigma}$ integral affine isomorphic to the first quadrant in $\mathbb{R}^{2}$.

Lemma 1.6. There is a one-to-one correspondence between toric blow-ups of $(Y, D)$ and refinements of $(B, \Sigma)$. Furthermore, if $(\tilde{Y}, \tilde{D})$ is a non-singular toric blow-up of $(Y, D)$, and $(\tilde{B}, \tilde{\Sigma})$ is the affine manifold with singularities constructed from $(\tilde{Y}, \tilde{D})$, then $\tilde{B}$ and $B$ are isomorphic as integral affine manifolds with singularities in such a way that $\tilde{\Sigma}$ is the corresponding refinement of $\Sigma$.

Proof. Let $\pi: \tilde{Y} \rightarrow Y$ be a toric blow-up. It follows from the condition that $\pi^{-1}(D)_{\text {red }}$ is an anti-canonical divisor that $\pi: \tilde{Y} \backslash \pi^{-1}(\operatorname{Sing}(D)) \rightarrow Y \backslash \operatorname{Sing}(D)$ is an isomorphism. Indeed, if this restriction of $\pi$ has an exceptional divisor, it must have discrepancy $a(E, Y, D)=-1$. But by [KM98, Cor. 2.31, (3), the smallest discrepancy occuring is 0 .

Thus necessarily $\pi$ is a blow-up along a subscheme supported on $\operatorname{Sing}(D)$. Let $x \in \operatorname{Sing}(D)$ be a double point of $D$, corresponding to a cone $\sigma \in \Sigma$. Note $\sigma$ can be viewed as a rational polyhedral cone defining a non-singular toric variety $X_{\sigma} \cong \mathbb{A}^{2}$. Then étale locally near $x$, the pair $(Y, D)$ is isomorphic to the pair $\left(X_{\sigma}, \partial X_{\sigma}\right)$. One can then check that in this local model, the only possible blow-ups satisfying the 
definition of toric blow-ups come from subdivisions of the cone $\sigma$, i.e., toric blow-ups of $X_{\sigma}$. Indeed, the exceptional divisors of toric blowups are the only divisors with discrepancy -1 . This gives the desired correspondence. The second statement is then easily checked.

Example 1.7. It is easy to see that if $Y$ is a non-singular toric surface and $D=\partial Y$ is the toric boundary of $D$, then in fact the affine structure on $B$ extends across the origin, identifying $(B, \Sigma)$ with $\left(M_{\mathbb{R}}, \Sigma_{Y}\right)$, where $\Sigma_{Y}$ is the fan for $Y$. Indeed, if $\rho_{j} \in \Sigma_{Y}$ corresponds to the divisor $D_{j}$ and $\rho_{j}=\mathbb{R}_{\geq 0} v_{j}$ with $v_{j} \in M$ primitive, then it is a standard fact that

$$
v_{i-1}+\left(D_{i}\right)^{2} v_{i}+v_{i+1}=0 .
$$

Since $Y$ is non-singular, there is always a linear identification $\varphi_{i}: M \stackrel{\sim}{\rightarrow} \mathbb{Z}^{2}$ taking $v_{i-1}$ to $(1,0), v_{i}$ to $(0,1)$, and thus $v_{i+1}$ must map to $\left(-1,-D_{i}^{2}\right)$. So on $U_{i}$, a chart for the affine structure on $B$ is $\psi_{i}^{\prime}=\varphi_{i}^{-1} \circ \psi_{i}: U_{i} \rightarrow M_{\mathbb{R}}$. The maps $\psi_{i}^{\prime}$ glue to give an integral affine isomorphism $B \rightarrow M_{\mathbb{R}}$.

In fact, the converse is also true:

Lemma 1.8. If the affine structure on $B_{0}=B \backslash\{0\}$ extends across the origin, then $Y$ is toric and $D=\partial Y$.

Proof. We first note that by Lemma 1.6, we can replace $(Y, D)$ with a non-singular toric blow-up without affecting the affine manifold $B$. By Proposition 1.3, we can thus assume the existence of a toric model $\pi:(Y, D) \rightarrow(\bar{Y}, \bar{D})$. If $\bar{D}_{i}$ is the image of $D_{i}$ under this map, then $\bar{D}_{i}^{2} \geq D_{i}^{2}$.

We first claim that $(Y, D)$ is isomorphic to $(\bar{Y}, \bar{D})$ if and only if equality holds for every $i$. Indeed, if equality holds for a given $i$, then $\pi$ can't contract any curves which intersect $D_{i}$. On the other hand, $\pi$ can't contract any curves contained in $Y \backslash D$ since then $D$ would not be an anti-canonical cycle.

Now assume that $(Y, D)$ is not toric, so that $\pi$ is not an isomorphism. Let $\left(M_{\mathbb{R}}, \bar{\Sigma}\right)$ be the fan for the toric pair $(\bar{Y}, \bar{D})$, with rays $\bar{\rho}_{1}, \ldots, \bar{\rho}_{n}$ corresponding to $\rho_{1}, \ldots, \rho_{n}$. In general, $B \backslash \rho_{1}$ has a coordinate chart $\psi: B \backslash \rho_{1} \rightarrow M_{\mathbb{R}}$, constructed by gluing together coordinate charts for $U_{2}, \ldots, U_{n}$. This can be done so that $\sigma_{1,2}$ is mapped to the cone of $\bar{\Sigma}$ generated by $\bar{\rho}_{1}$ and $\bar{\rho}_{2}$. It is now enough to show the following:

Claim. For suitable choice of $\rho_{1}$, in fact $\psi$ is injective and $\psi\left(B \backslash \rho_{1}\right)$ is strictly contained in $M_{\mathbb{R}} \backslash \bar{\rho}_{1}$.

To show this, first let us analyze the effects of one blow-up on these charts. Let $(Y, D) \rightarrow\left(Y^{\prime}, D^{\prime}\right)$ be given by a blow-up of a single point $p \in D_{i}^{\prime}$ for some $i$, where $\left(Y^{\prime}, D^{\prime}\right)$ is obtained from $(\bar{Y}, \bar{D})$ by a sequence of blow-ups with centers at smooth 
points of the boundary. Let $\left(B^{\prime}, \Sigma^{\prime}\right)$ be the tropicalization of $\left(Y^{\prime}, D^{\prime}\right)$. Let us examine the difference between the charts $\psi: B \backslash \rho_{1} \rightarrow M_{\mathbb{R}}$ and $\psi^{\prime}: B^{\prime} \backslash \rho_{1}^{\prime} \rightarrow M_{\mathbb{R}}$ defined as above. If $i=1$, then $B \backslash \rho_{1}$ and $B^{\prime} \backslash \rho_{1}^{\prime}$ are affine isomorphic and $\psi, \psi^{\prime}$ agree. Otherwise, let $\sigma_{1, i}=\bigcup_{j=2}^{i} \sigma_{j-1, j} \subset B$, with $\sigma_{1, i}^{\prime} \subset B^{\prime}$ defined similarly. Then $\sigma_{1, i}$ and $\sigma_{1, i}^{\prime}$ are affine isomorphic and $\left.\psi\right|_{\sigma_{1, i} \backslash \rho_{1}}=\left.\psi^{\prime}\right|_{\sigma_{1, i}^{\prime} \backslash \rho_{1}}$. On the other hand, $\left.\psi\right|_{B \backslash \sigma_{1, i}}=\left.T_{i} \circ \psi^{\prime}\right|_{B^{\prime} \backslash \sigma_{1, i}^{\prime}}$ where $T_{i}: M_{\mathbb{R}} \rightarrow M_{\mathbb{R}}$ is the shear $T_{i}(m)=m+\left\langle m, n_{i}^{\prime}\right\rangle v_{i}^{\prime}$, where $v_{i}^{\prime}$ is a primitive generator of $\psi^{\prime}\left(\rho_{i}^{\prime}\right)$ and $n_{i}^{\prime} \in N$ is primitive, annihilates $v_{i}^{\prime}$, and is positive on $\psi^{\prime}\left(\sigma_{i, i+1}^{\prime}\right)$.

Now note that $\bar{D}_{i}^{2}>D_{i}^{2}$ for at least one $i$, and by choosing $\rho_{1}$ appropriately, we can assume that this is the case for some $i \neq 1$. Furthermore, we can also assume that if there is a $\bar{\rho}_{j} \in \bar{\Sigma}$ with $\bar{\rho}_{j}=-\bar{\rho}_{1}$, then there is an $i$ with $\bar{D}_{i}^{2}>D_{i}^{2}$ with $i \neq 1, j$. Applying the above description of the change of the coordinate charts under one blow-up repeatedly then shows the claim.

Now if the affine structure on $B_{0}$ extended across the origin, then $\psi$ would extend to an isomorphism $\psi: B \rightarrow M_{\mathbb{R}}$, which contradicts the claim.

Example 1.9. Let $Y$ be a del Pezzo surface of degree 5. Thus $Y$ is isomorphic to the blowup of $\mathbb{P}^{2}$ in 4 points in general position. The surface $Y$ contains exactly 10 $(-1)$-curves. It is easy to find an anti-canonical cycle $D$ of length 5 among these 10 curves.

In this case, consider $B \backslash \rho_{1}$. Each chart $\psi_{i}: U_{i} \rightarrow M_{\mathbb{R}}$ can be composed with an integral linear function on $M_{\mathbb{R}}$ in such a way that the charts $\psi_{2}, \psi_{3}, \psi_{4}$ and $\psi_{5}$ glue to give a chart $\psi: B \backslash \rho_{1} \rightarrow M_{\mathbb{R}}$. This can be done, for example, with

$\psi\left(v_{1}\right)=(1,0), \quad \psi\left(v_{2}\right)=(0,1), \quad \psi\left(v_{3}\right)=(-1,1), \quad \psi\left(v_{4}\right)=(-1,0), \quad \psi\left(v_{5}\right)=(0,-1)$.

We can then take a chart $\psi^{\prime}: U_{5} \cup U_{1} \rightarrow M_{\mathbb{R}}$ which agrees with $\psi$ on $\sigma_{5,1}$, and hence takes the values

$$
\psi^{\prime}\left(v_{5}\right)=(0,-1), \quad \psi^{\prime}\left(v_{1}\right)=(1,-1), \quad \psi^{\prime}\left(v_{2}\right)=(1,0),
$$

see Figure 1.1.

Thus $B$, as an affine manifold, can be constructed by cutting $M_{\mathbb{R}}$ along the positive real axis, and then identifying the two copies of the cone $\sigma_{1,2}$ via an integral linear transformation.

Example 1.10. Suppose given a Looijenga pair $(Y, D)$ with $D_{i}^{2} \leq-2$ for all $i$ and $D$ is negative definite (which is equivalent to $D_{i}^{2} \leq-3$ for some $i$ ). Then we have an analytic contraction $p: Y \rightarrow \bar{Y}$ with $\bar{Y}$ having a single cusp singularity. This case will lead to our proof of Looijenga's conjecture. We can describe $(B, \Sigma)$ as follows. Let $M \cong \mathbb{Z}^{2}$ and take $v_{0}, v_{1}$ to be a basis for $M$, and define $v_{i}$ for $i \in \mathbb{Z}$ by the relation

$$
v_{i-1}+\left(D_{i \bmod n}^{2}\right) v_{i}+v_{i+1}=0 .
$$




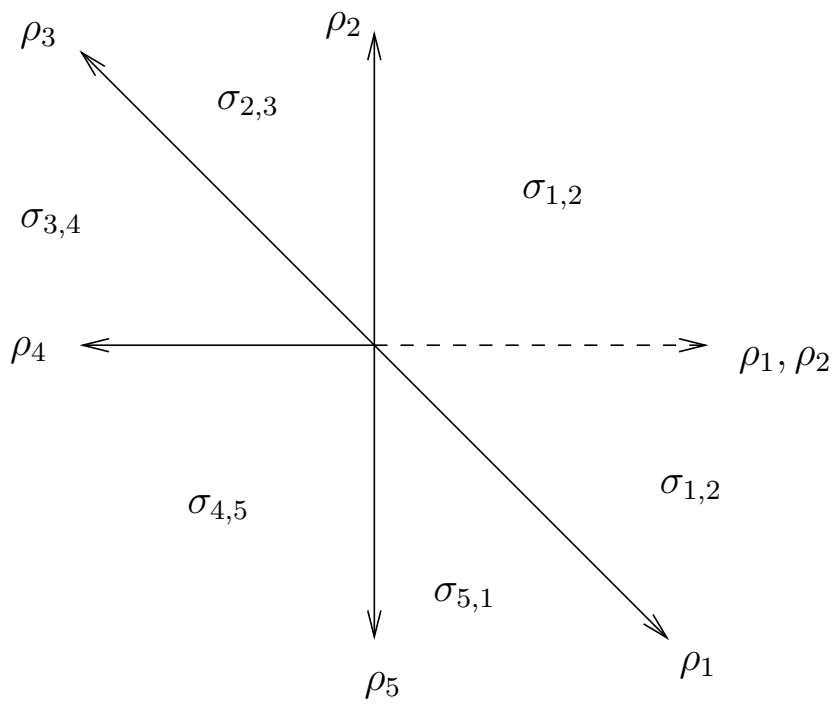

FIGURE 1.1.

We define an infinite fan $\tilde{\Sigma}$ in $M_{\mathbb{R}}$ whose two-dimensional cones are the cones generated by $v_{i}$ and $v_{i+1}, i \in \mathbb{Z}$. It is easy to check that these cones do indeed form a fan and that the support of the fan $|\tilde{\Sigma}|$ is a strictly convex cone. If we define $T \in \operatorname{SL}(M)$ by $T\left(v_{0}\right)=v_{n}$ and $T\left(v_{1}\right)=v_{n+1}$, then $T\left(v_{i}\right)=v_{i+n}$ for each $i$. Necessarily $T$ takes $|\tilde{\Sigma}|$ to itself, so the boundary rays of the closure of $|\tilde{\Sigma}|$ are real eigenspaces for $T$. Hence $T$ is hyperbolic, i.e., $\operatorname{Tr} T>2$.

We now obtain $(B, \Sigma)$ by dividing out $|\tilde{\Sigma}|$ by the action of $T$.

1.3. The Mumford degeneration and Givental's construction. The toric case of Theorem 0.1 yields Givental's construction for mirrors of toric varieties in the surface case, and can also be seen as a special case of a construction due to Mumford [Mum]. Mumford's construction in general produces degenerations of arbitrary toric varieties; the construction as we review it here only gives degenerations of the algebraic torus. This should be regarded as a warmup for our general construction.

A toric monoid $P$ is a (commutative) monoid whose Grothendieck group $P^{\text {gp }}$ is a finitely generated free abelian group and $P=P^{\mathrm{gp}} \cap \sigma_{P}$, where $\sigma_{P} \subseteq P^{\mathrm{gp}} \otimes_{\mathbb{Z}} \mathbb{R}$ is a convex rational polyhedral cone. Let $M=\mathbb{Z}^{n}$ be a lattice, for some arbitrary rank $n$. Fix a fan $\Sigma$ in $M_{\mathbb{R}}=M \otimes_{\mathbb{Z}} \mathbb{R}$, whose support, $|\Sigma|$, is convex. In what follows, we view $B=|\Sigma|$ as an affine manifold with boundary. We denote by $\Sigma_{\max }$ the set of maximal cones in $\Sigma$.

We now generalize the usual notion of a convex piecewise linear function on a fan. If one is interested in $\mathbb{R}$-valued convex functions, then one can take $P=\mathbb{N}, \sigma_{P}=\mathbb{R}_{\geq 0}$. Then the following definition yields the notion of a piecewise linear $\mathbb{R}$-valued function 
with integral slopes, and convexity here means upper convexity, i.e., the function is the supremum of a collection of linear functions.

Definition 1.11. A $\Sigma$-piecewise linear function $\varphi:|\Sigma| \rightarrow P_{\mathbb{R}}^{\mathrm{gp}}$ is a continuous function such that for each $\sigma \in \Sigma_{\max },\left.\varphi\right|_{\sigma}$ is given by an element $\varphi_{\sigma} \in \operatorname{Hom}_{\mathbb{Z}}\left(M, P^{\mathrm{gp}}\right)=$ $N \otimes_{\mathbb{Z}} P^{\mathrm{gp}}$.

For each codimension one cone $\rho \in \Sigma$ contained in two maximal cones $\sigma_{+}, \sigma_{-} \in \Sigma_{\max }$, we can write

$$
\varphi_{\sigma_{+}}-\varphi_{\sigma_{-}}=n_{\rho} \otimes \kappa_{\rho, \varphi}
$$

where $n_{\rho} \in N$ is the unique primitive element annihilating $\rho$ and positive on $\sigma_{+}$, and $\kappa_{\rho, \varphi} \in P^{\mathrm{gp}}$. We call $\kappa_{\rho, \varphi}$ the bending parameter. Note (as the notation suggests) it depends only on the codimension one cone $\rho$ (not on the ordering of $\sigma_{+}, \sigma_{-}$).

We say a $\Sigma$-piecewise linear function $\varphi:|\Sigma| \rightarrow P^{\text {gp }}$ is $P$-convex (strictly $P$-convex) if for every codimension one cone $\rho \in \Sigma, \kappa_{\rho, \varphi} \in P\left(\kappa_{\rho, \varphi} \in P \backslash P^{\times}\right.$, where $P^{\times}$is the group of invertible elements of $P$ ).

Example 1.12. Take a complete fan $\Sigma$ in $M_{\mathbb{R}}$. This defines a toric variety $Y=Y_{\Sigma}$, which we assume is non-singular. We let $P \subset P^{\text {gp }}$ be given by the cone of effective curves,

$$
\mathrm{NE}(Y) \subset A_{1}(Y, \mathbb{Z})
$$

Each codimension one cone $\rho \in \Sigma$ corresponds to a one-dimensional toric stratum $D_{\rho} \subset \partial Y$, hence a class $\left[D_{\rho}\right] \in \mathrm{NE}(Y)=P$. If $\omega \in \Sigma(1)$, the set of rays of $\Sigma$, we also write $D_{\omega}$ for the corresponding toric divisor.

Lemma 1.13. Define $s: T_{\Sigma}:=\mathbb{Z}^{\Sigma(1)} \rightarrow M$ to send the basis element $t_{\omega}, \omega \in \Sigma(1)$ to the first lattice point $m_{\omega}$ on $\omega$. Then

$$
A_{1}(Y, \mathbb{Z}) \ni \beta \mapsto \sum_{\omega \in \Sigma(1)}\left(D_{\omega} \cdot \beta\right) t_{\omega}
$$

identifies $A_{1}(Y, \mathbb{Z})$ with $\operatorname{Ker}(s)$, giving rise to an exact sequence

$$
0 \rightarrow A_{1}(Y, \mathbb{Z}) \rightarrow T_{\Sigma} \stackrel{s}{\rightarrow} M \rightarrow 0
$$

Then there is a unique $\Sigma$-piecewise linear section $\tilde{\varphi}: M \rightarrow T_{\Sigma}$ satisfying $\tilde{\varphi}\left(m_{\omega}\right)=t_{\omega}$. Let $\pi: T_{\Sigma} \rightarrow A_{1}(Y, \mathbb{Z})$ be any splitting, and set $\varphi:=\pi \circ \tilde{\varphi}$. Then $\varphi: M \rightarrow A_{1}(Y, \mathbb{Z})=$ $P^{\mathrm{gp}}$ is $\Sigma$-piecewise linear and strictly $P$-convex, with

$$
\kappa_{\rho, \varphi}=\left[D_{\rho}\right]
$$

for each codimension one cone $\rho \in \Sigma$. Up to a linear function, $\varphi$ is the unique $\Sigma$ piecewise linear map with these bending parameters. 
Proof. The exact sequence is standard. Since $\Sigma$ is a complete non-singular fan, it is clear that there exists such a unique $\tilde{\varphi}$. To calculate the kink along a codimension one $\rho \in \Sigma$, suppose $\rho$ is generated by basis vectors $e_{1}, \ldots, e_{n-1}$ and $\rho$ is contained in two maximal cones, generated by $e_{1}, \ldots, e_{n}$ and $e_{1}, \ldots, e_{n-1}, e_{n}^{\prime}:=-e_{n}+\sum_{i=1}^{n-1} a_{i} e_{i}$. Let $t_{1}, \ldots, t_{n}, t_{n}^{\prime}$ be the generators of $T_{\Sigma}$ mapping to $e_{1}, \ldots, e_{n}, e_{n}^{\prime}$ respectively. Then the kink is $\kappa_{\rho, \tilde{\varphi}}=t_{n}+t_{n}^{\prime}-\sum_{i=1}^{n-1} a_{i} t_{i}$. On the other hand, if $D_{1}, \ldots, D_{n}, D_{n}^{\prime}$ are the divisors corresponding to the rays generated by $e_{1}, \ldots, e_{n}, e_{n}^{\prime}$ respectively, then $D_{n} \cdot D_{\rho}=D_{n}^{\prime} \cdot D_{\rho}=1$ and using the rational function $z^{e_{i}^{*}}, D_{i}$ is linearly equivalent to $-a_{i} D_{n}^{\prime}$ plus a sum of toric divisors disjoint from $D_{\rho}$. Thus $D_{i} \cdot D_{\rho}=-a_{i}$ and we see that $\kappa_{\rho, \tilde{\varphi}}$ is the image of $\left[D_{\rho}\right]$ under the inclusion $A_{1}(Y, \mathbb{Z}) \rightarrow T_{\Sigma}$. Thus $\kappa_{\pi \circ \tilde{\varphi}, \rho}=$ $\pi\left(\kappa_{\tilde{\varphi}, \rho}\right)=\left[D_{\rho}\right]$ as required.

Given a $\Sigma$-piecewise linear and $P$-convex function $\varphi:|\Sigma| \rightarrow P^{\mathrm{gp}}$ we can define a monoid $P_{\varphi} \subset M \times P^{\text {gp }}$ by

$$
P_{\varphi}:=\{(m, \varphi(m)+p)|m \in| \Sigma \mid, p \in P\} .
$$

This is the set of integral points lying above the graph of $\varphi$, in the sense given by the partial order on $P^{\mathrm{gp}}$ defined by $p_{1} \geq p_{2}$ if $p_{1}-p_{2} \in P$. The convexity of $\varphi$ is equivalent to $P_{\varphi}$ being closed under addition. Furthermore, we have a natural inclusion $P \hookrightarrow P_{\varphi}$ given by $p \mapsto(0, p)$, which gives us a morphism

$$
f: \operatorname{Spec} \mathbb{k}\left[P_{\varphi}\right] \rightarrow \operatorname{Spec} \mathbb{k}[P] .
$$

This morphism is flat as $\mathbb{k}\left[P_{\varphi}\right]$ is freely generated as a $\mathbb{k}[P]$-module by all elements of the form $z^{(m, \varphi(m))}, m \in|\Sigma|$. It is easy to see that a general fibre of $f$ is isomorphic to the algebraic torus $\operatorname{Spec} \mathbb{k}[M]$ : in fact, if we consider the big torus orbit $U=\operatorname{Spec} \mathbb{k}\left[P^{\mathrm{gp}}\right] \subset$ Spec $\mathbb{k}[P], f^{-1}(U)=U \times \operatorname{Spec} \mathbb{k}[M]$.

We now describe the fibres over other toric strata of $\operatorname{Spec} \mathbb{k}[P]$. Let $x \in \operatorname{Spec} \mathbb{k}[P]$ be a point in the torus orbit corresponding to a face $Q \subset P$. Then by replacing $P$ with the localized monoid $P-Q$ obtained by inverting all elements of $Q$, we may assume that $x$ is contained in the smallest toric stratum of Spec $\mathbb{k}[P]$. Consider the composed map

$$
\bar{\varphi}:|\Sigma| \stackrel{\varphi}{\longrightarrow} P^{\mathrm{gp}} \rightarrow P^{\mathrm{gp}} / P^{\times}
$$

Note $\bar{\varphi}$ is also piecewise linear. Let $\bar{\Sigma}$ be the fan (of convex but not necessarily strictly convex cones) whose maximal cones are the maximal domains of linearity of $\bar{\varphi}$. Then $f^{-1}(x)$ can be written as

$$
f^{-1}(x)=\operatorname{Spec} \mathbb{k}[\bar{\Sigma}] .
$$


Here,

$$
\mathbb{k}[\bar{\Sigma}]=\bigoplus_{m \in M \cap|\Sigma|} \mathbb{k} z^{m}
$$

with multiplication given by

$$
z^{m} \cdot z^{m^{\prime}}= \begin{cases}z^{m+m^{\prime}} & \text { if } m, m^{\prime} \text { lie in a common cone of } \bar{\Sigma} \\ 0 & \text { otherwise. }\end{cases}
$$

In particular, the irreducible components of $f^{-1}(x)$ are the toric varieties $\operatorname{Spec} \mathbb{k}[\sigma \cap M]$ for $\sigma \in \bar{\Sigma}_{\max }$.

In the particular case that $\operatorname{rank} M=2$ and $\Sigma$ defines a non-singular complete surface with $n$ toric divisors, suppose $\varphi$ is strictly convex. If $x$ is a point of the smallest toric stratum of Spec $\mathbb{k}[P]$, then $f^{-1}(x)$ is just $\mathbb{V}_{n} \subset \mathbb{A}^{n}$, the reduced cyclic union of coordinate $\mathbb{A}^{2}$ 's:

$$
\mathbb{V}_{n}=\mathbb{A}_{x_{1}, x_{2}}^{2} \cup \mathbb{A}_{x_{2}, x_{3}}^{2} \cup \cdots \cup \mathbb{A}_{x_{n}, x_{1}}^{2} \subset \mathbb{A}_{x_{1}, \ldots, x_{n}}^{n}
$$

We call $\mathbb{V}_{n}$ the vertex, or more specifically, the $n$-vertex.

We will need in the sequel the degenerate case of the $n$-vertex for $n=2$. This is a union of two affine planes and can be described as the double cover

$$
\mathbb{V}_{2}=\operatorname{Spec} \mathbb{K}\left[x_{1}, x_{2}, y\right] /\left(y^{2}-x_{1}^{2} x_{2}^{2}\right)=\mathbb{A}_{x_{1}, x_{2}}^{2} \cup \mathbb{A}_{x_{2}, x_{1}}^{2} .
$$

Of course, this does not appear as a central fibre of a Mumford degeneration. Analogously, one can define

$$
\mathbb{V}_{1}=\operatorname{Spec} \mathbb{k}[x, y, z] /\left(x y z-x^{2}-z^{3}\right),
$$

the affine cone over a nodal curve embedded in weighted projective space $W \mathbb{P}^{3}(3,1,2)$.

Example 1.14. In Example 1.12, with the choice of $\varphi$ given by Lemma 1.13, the family

$$
\operatorname{Spec} \mathbb{k}\left[P_{\varphi}\right] \rightarrow \operatorname{Spec} \mathbb{k}[\mathrm{NE}(Y)]
$$

in fact gives the family of mirror manifolds to the toric variety $Y$, as constructed by Givental Giv.

In fact, the mirror of a toric variety also includes the data of a Landau-Ginzburg potential, which is a regular function. If $Y$ is Fano, the potential is

$$
W=\sum_{\rho} z^{\left(m_{\rho}, \varphi\left(m_{\rho}\right)\right)}
$$

where we sum over all rays $\rho \in \Sigma$, and $m_{\rho} \in M$ denotes the primitive generator of $\rho$. If $Y$ is not Fano, the potential receives corrections which can be viewed as coming from degenerate holomorphic disks on $Y$ with irreducible components mapping into $D$. 


\section{Modified Mumford DEgenerations}

In this section, we fix $(Y, D)$ a Looijenga pair, and let $(B, \Sigma)$ be the tropicalisation of $(Y, D)$ defined in $\$ 1.2$. The fan $\Sigma$ contains rays $\rho_{1}, \ldots, \rho_{n}$ corresponding to divisors $D_{1}, \ldots, D_{n}$, ordered cyclically. As usual, we write the two-dimensional cones of $\Sigma$ as $\sigma_{i, i+1}$ being the cone with edges $\rho_{i}$ and $\rho_{i+1}$, with indices taken modulo $n$.

We explain how to generalize Mumford's degeneration, to give a canonical formal deformation of $\mathbb{V}_{n}^{o}=\mathbb{V}_{n} \backslash\{0\}$ associated to $(Y, D)$ if $n \geq 3$. Locally on $B_{0}$ the picture is toric and we have Mumford's degenerations described in \$1.3. As Mumford's construction is functorial, the deformations built locally patch together canonically: this is a minor variation on the ideas of [GS07]. In particular, §\$2.1 and [2.2 are variations of ideas in GS07. However, it differs crucially in several respects which prevent us from just referring to GS07]. First, we work with piecewise linear functions with values in a vector space $P_{\mathbb{R}}^{\mathrm{gp}}$ rather than just $\mathbb{R}$. This allows us to construct higher dimensional formal families, namely over the completion of Spec $\mathbb{k}[P]$ at the zerodimensional stratum. Second, by avoiding a description of local models in codimension at least two, we avoid some of the technical complexities of [GS07].

Here are the details.

2.1. The uncorrected degeneration. We fix some notation. For any locally constant sheaf $\mathcal{F}$ on $B_{0}$, and any simply connected subset $\tau \subset B_{0}$ we write $\mathcal{F}_{\tau}$ for the stalk of this local system at any point of $\tau$ (as any two such stalks are canonically identified by parallel transport). In particular, we apply this for the sheaf $\Lambda$ of integral constant vector fields, as well as for the sheaf $\Lambda_{\mathbb{R}}:=\Lambda \otimes_{\mathbb{Z}} \mathbb{R}$.

For each cone $\tau \in \Sigma$ with $\operatorname{dim} \tau=1$ or 2 , we write $\tau^{-1} \Sigma$ for the localized fan of convex (but not strictly convex) cones in $\Lambda_{\tau, \mathbb{R}}$ described as follows. If $\operatorname{dim} \tau=2$, then $\tau^{-1} \Sigma$ just consists of the single cone $\Lambda_{\tau, \mathbb{R}}$. If $\operatorname{dim} \tau=1$, then $\tau^{-1} \Sigma$ consists of three cones: the tangent line to $\tau$ and the two half-spaces bounded by the tangent line to $\tau$.

Let $P \subseteq P^{\text {gp }}$ be a toric monoid as in $\$ 1.3$.

Definition 2.1. A ( $P_{\mathbb{R}}^{\mathrm{gp}}$-valued) $\Sigma$-piecewise linear multivalued function on $B$ is a collection $\varphi=\left\{\varphi_{i}\right\}$ with $\varphi_{i}$ a $\Sigma$-piecewise linear function on $U_{i}$ with values in $P_{\mathbb{R}}^{\text {gp }}$.

Note this is equivalent to giving a $\rho_{i}^{-1} \Sigma$-piecewise linear function $\varphi_{i}: \Lambda_{\mathbb{R}, \rho_{i}} \rightarrow P_{\mathbb{R}}^{\mathrm{gp}}$ for each ray $\rho_{i} \in \Sigma$. Two such functions $\varphi, \varphi^{\prime}$ are said to be equivalent if $\varphi_{i}-\varphi_{i}^{\prime}$ is linear for each $i$. Note the equivalence class of $\varphi$ is determined by the collection of bending parameters $\kappa_{\rho, \varphi} \in P^{\mathrm{gp}}$. We say the function is convex (strictly convex) if $\kappa_{\rho, \varphi} \in P\left(\kappa_{\rho, \varphi} \in P \backslash P^{\times}\right)$for each $\rho$.

We drop the modifiers $\Sigma$ and $P$ when they are clear from context. 
Construction 2.2. The collection $\left\{\varphi_{i}\right\}$ determines a local system $\mathcal{P}$ on $B_{0}$ as follows. First, we can construct an affine manifold $\mathbb{P}_{0}$ which comes along with the structure of $P_{\mathbb{R}}^{\mathrm{gp}}$-principal bundle $\pi: \mathbb{P}_{0} \rightarrow B_{0}$ and a piecewise linear section $\varphi: B_{0} \rightarrow \mathbb{P}_{0}$ as follows: we glue $U_{i} \times P_{\mathbb{R}}^{\mathrm{gp}}$ to $U_{i+1} \times P_{\mathbb{R}}^{\mathrm{gp}}$ along $\left(U_{i} \cap U_{i+1}\right) \times P_{\mathbb{R}}^{\mathrm{gp}}$ by

$$
(x, p) \rightarrow\left(x, p+\varphi_{i+1}(x)-\varphi_{i}(x)\right) .
$$

By construction we have local sections $x \mapsto\left(x, \varphi_{i}(x)\right)$ which patch to give a piecewise linear section $\varphi$. One checks immediately the isomorphism class (of the $P_{\mathbb{R}}^{\mathrm{gp}}$-principal bundle together with the section) depends only on the equivalence class of $\left\{\varphi_{i}\right\}$. The bundle $\mathbb{P}_{0} \rightarrow B_{0}$ can be viewed as a tropical analogue of a sum of line bundles, and $\left\{\varphi_{i}\right\}$ yield a section of this vector bundle. Convexity is analogous to holomorphicity of the section.

We then define

$$
\mathcal{P}:=\pi_{*} \Lambda_{\mathbb{P}_{0}} \cong \varphi^{-1} \Lambda_{\mathbb{P}_{0}}
$$

on $B_{0}$. We have an exact sequence

$$
0 \rightarrow \underline{P}^{\mathrm{gp}} \rightarrow \mathcal{P} \stackrel{r}{\longrightarrow} \Lambda \rightarrow 0
$$

of local systems on $B_{0}$, where $r$ is the derivative of $\pi$.

Note over $U_{i}$, the description of $\mathbb{P}_{0}$ as $U_{i} \times P_{\mathbb{R}}^{\mathrm{gp}}$ gives a splitting $\left.\left.\mathcal{P}\right|_{U_{i}} \cong \Lambda\right|_{U_{i}} \times P^{\mathrm{gp}}$.

Example 2.3. Our standard example, fundamental to this paper, will be as follows. Suppose $P$ is a monoid which comes with a homomorphism $\eta: \mathrm{NE}(Y) \rightarrow P$ of monoids.

Choose $\varphi$ by specifying $\varphi_{i}$ on $U_{i}$ by the formula

$$
\kappa_{\rho_{i}, \varphi_{i}}=\eta\left(\left[D_{i}\right]\right) .
$$

Such a $\varphi$ is well-defined up to linear functions, and always exists. This is always convex, and is strictly convex provided $\eta\left(\left[D_{i}\right]\right)$ is not invertible for any $i$.

Now suppose given a piecewise linear multivalued $P$-convex function $\varphi$ on $B$. We explain how Mumford's construction determines a canonical formal deformation of $\mathbb{V}_{n}^{o}$, restricting to the case $n \geq 3$ for ease of exposition.

For each $\tau \in \Sigma$ with $\operatorname{dim} \tau>0, \varphi$ determines a canonically defined $\tau^{-1} \Sigma$-piecewise linear section $\varphi_{\tau}: \Lambda_{\mathbb{R}, \tau} \rightarrow \mathcal{P}_{\mathbb{R}, \tau}$ of the projection $\mathcal{P}_{\mathbb{R}, \tau} \rightarrow \Lambda_{\mathbb{R}, \tau}$. If $U_{i} \cap \tau \neq \emptyset$, we use the representative $\varphi_{i}$ on $U_{i}$ and extend it linearly on each cone in the fan $\tau^{-1} \Sigma$ to obtain a $P$-convex piecewise linear function on $\tau^{-1} \Sigma$, which we also write as $\varphi_{i}$. Then the section $\varphi_{\tau}$ is defined as in Construction 2.2 by $x \mapsto\left(x, \varphi_{i}(x)\right)$, using the splitting $\mathcal{P}_{\mathbb{R}, \tau}=\Lambda_{\mathbb{R}, \tau} \times P_{\mathbb{R}}^{\mathrm{gp}}$. We note a different choice of representative of $\varphi_{i}$ leads to a different choice of splitting and the same section $\varphi_{\tau}$, so this section is well-defined. 
Now define the toric monoid $P_{\varphi_{\tau}} \subset \mathcal{P}_{\tau}$ by

$$
P_{\varphi_{\tau}}:=\left\{q \in \mathcal{P}_{\tau} \mid q=p+\varphi_{\tau}(m) \text { for some } p \in P, m \in \Lambda_{\tau}\right\} \text {. }
$$

By the definition of convexity of $\varphi$, we have canonical inclusions

$$
P_{\varphi_{\rho}} \subset P_{\varphi_{\sigma}} \subset \mathcal{P}_{\rho}
$$

whenever $\rho \subset \sigma \in \Sigma$. If $\rho \in \Sigma$ is a ray with $\rho \subset \sigma_{ \pm} \in \Sigma_{\max }$ we have the equality

$$
P_{\varphi_{\sigma_{+}}} \cap P_{\varphi_{\sigma_{-}}}=P_{\varphi_{\rho}}
$$

Definition 2.4 (Monomial ideals). A (monoid) ideal of a monoid $P$ is a subset $I \subset P$ such that $p \in I, q \in P$ implies $p+q \in I$. An ideal determines a monomial ideal in the monoid ring $\mathbb{k}[P]$, generated by monomials $z^{p}$ for $p \in I$. We also denote this ideal by $I$, hopefully with no confusion. As a consequence, we shall sometimes write certain ideal operations either additively or multiplicatively, i.e., for $J \subset P$,

$$
k J=\left\{p_{1}+\cdots+p_{k} \mid p_{i} \in J, 1 \leq i \leq k\right\},
$$

and the corresponding monomial ideal is $J^{k}$.

Let $\mathfrak{m}=P \backslash P^{\times}$. This is the unique maximal ideal of $P$, defining a monomial ideal $\mathfrak{m} \subset \mathbb{k}[P]$. Note $\mathbb{k}[P] / \mathfrak{m} \cong \mathbb{k}\left[P^{\times}\right]$.

We say an ideal $I \subset P$ is $\mathfrak{m}$-primary if

$$
\mathfrak{m}=\sqrt{I}:=\{p \in P \mid \text { there exists a positive integer } k \text { such that } k p \in I\},
$$

in which case the same holds for the associated monomial ideal $I \subset \mathbb{k}[P]$.

Recall from $\$ 1.3$ that we are only considering toric monoids $P$, i.e., monoids which are the intersection of rational polyhedral cones $\sigma_{P}$ with lattices. Such monoids are always finitely generated, so that $\mathbb{k}[P]$ is Noetherian. If $\sigma_{P}$ is strictly convex, then $\mathfrak{m}$ is the maximal ideal corresponding to the unique torus fixed point of $\operatorname{Spec} \mathbb{k}[P]$.

Fix an ideal $I \subset P$, and recalling that we write $R=\mathbb{k}[P]$, set

$$
R_{I}:=\mathbb{k}[P] / I \text {. }
$$

We define for $\tau \in \Sigma$, $\operatorname{dim} \tau>0$, the ring

$$
R_{\tau, I}:=\mathbb{k}\left[P_{\varphi_{\tau}}\right] \otimes_{R} R_{I},
$$

noting that $P$ acts naturally on $P_{\varphi_{\tau}}$ by addition. So Spec $R_{\tau, I}$ is a base-change of the Mumford degeneration induced by $\varphi_{\tau}$ on the localized fan $\tau^{-1} \Sigma$.

One observes 
Proposition 2.5. Let $v_{i}$ denote the primitive generator of the tangent ray to $\rho_{i}$, for each $i$. Then viewing $z^{\kappa_{\rho, \varphi}} \in \mathbb{k}[P]$ as determining an element in $R_{I}$, we have

$$
\frac{R_{I}\left[X_{i-1}, X_{i}^{ \pm}, X_{i+1}\right]}{\left(X_{i-1} X_{i+1}-z^{\kappa_{\rho_{i}, \varphi}} X_{i}^{-D_{\rho_{i}}^{2}}\right)} \cong R_{\rho_{i}, I}
$$

via the map

$$
X_{j} \mapsto z^{\varphi_{\rho_{i}}\left(v_{j}\right)}, \quad j \in\{i-1, i, i+1\} .
$$

Furthermore, there are natural maps

$$
\psi_{\rho_{i},-}: R_{\rho_{i}, I} \rightarrow R_{\sigma_{i-1, i}, I}, \quad \psi_{\rho_{i},+}: R_{\rho_{i}, I} \rightarrow R_{\sigma_{i, i+1}, I}
$$

induced by the inclusions $P_{\varphi_{\rho_{i}}} \subseteq P_{\varphi_{\sigma_{ \pm}}}$which induce isomorphisms

$$
\left(R_{\rho_{i}, I}\right)_{X_{i-1}} \cong R_{\sigma_{i-1, i}, I}, \quad\left(R_{\rho_{i}, I}\right)_{X_{i+1}} \cong R_{\sigma_{i, i+1}, I} .
$$

Proof. We need to check that the ideal on the left-hand side is mapped to zero, as the rest is obvious. Note by construction of $B, v_{i-1}+D_{\rho_{i}}^{2} v_{i}+v_{i+1}=0$ as elements of $\Lambda_{\rho_{i}}$, so one sees in fact that $\varphi_{\rho_{i}}\left(v_{i-1}\right)+\varphi_{\rho_{i}}\left(v_{i+1}\right)=\kappa_{\rho_{i}, \varphi}-D_{\rho_{i}}^{2} \varphi_{\rho_{i}}\left(v_{i}\right)$. The result then follows easily.

Remark 2.6. Since Spec $R_{\rho_{i}, I} \rightarrow$ Spec $R_{I}$ is a base-change of the Mumford degeneration, we can in fact say what a fibre of this morphism is over a closed point $x$ in the smallest toric stratum of Spec $\mathbb{k}[P]$, i.e., a point in Spec $R_{\mathfrak{m}}$. This depends on whether $\kappa_{\rho_{i}, \varphi} \in P$ is invertible or not. If it is not invertible, then the fibre is $\operatorname{Spec} \mathbb{k}\left[\rho_{i}^{-1} \Sigma\right] \cong$ Spec $\mathbb{k}\left[X_{i-1}, X_{i+1}, X_{i}^{ \pm 1}\right] /\left(X_{i-1} X_{i+1}\right)$. If $\kappa_{\rho_{i}, \varphi}$ is invertible, then the fibre is $\operatorname{Spec} \mathbb{k}\left[\mathbb{Z}^{2}\right]$. In this latter case, if $\rho_{i} \subset \sigma$, in fact the map $R_{\rho_{i}, I} \rightarrow R_{\sigma, I}$ induced by the inclusion $P_{\varphi_{\rho_{i}}} \subseteq P_{\varphi_{\sigma}}$ is an isomorphism.

Somewhat more generally, if $J \subset P$ is a radical ideal with $\kappa_{\rho_{i}, \varphi} \in J$, then in fact $R_{\rho_{i}, J} \cong \operatorname{Spec} R_{J}\left[\rho_{i}^{-1} \Sigma\right]$.

For $\tau \in \Sigma, \operatorname{dim} \tau \geq 1$, set

$$
U_{\tau, I}:=\operatorname{Spec} R_{\tau, I} .
$$

The maps $\psi_{\rho_{i}, \pm}$ induce open immersions $U_{\sigma_{i-1, i}, I} \hookrightarrow U_{\rho_{i}, I}$ and $U_{\sigma_{i, i+1}} \hookrightarrow U_{\rho_{i}, I}$. Denoting the image of each of these immersions as $U_{\rho_{i}, \sigma_{i-1, i}, I}$ and $U_{\rho_{i}, \sigma_{i, i+1}, I}$ respectively, we note that

$$
U_{\rho_{i}, \sigma_{i-1, i}, I} \cap U_{\rho_{i}, \sigma_{i, i+1}, I} \cong \operatorname{Spec}\left(R_{\rho_{i}, I}\right)_{X_{i-1} X_{i+1}} \cong\left(\mathbb{G}_{m}\right)^{2} \times \operatorname{Spec}\left(R_{I}\right)_{z^{\kappa} \rho_{i}, \varphi}
$$

Note that if $\kappa_{\rho_{i}, \varphi} \in \sqrt{I}$ then the localization $(\mathbb{k}[P] / I)_{z^{\kappa} \rho_{i}, \varphi}$ is zero, and the intersection is empty.

We can now define our analogue of the Mumford degeneration. 
Construction 2.7. Suppose that the number of irreducible components $n$ of $D$ satisfies $n \geq 3$, that $\varphi$ is a PL multivalued function, and $I \subset P$ an ideal such that $\kappa_{\rho, \varphi} \in \sqrt{I}$ for all rays $\rho \in \Sigma$. Then there are canonical identifications of open subsets

$$
U_{\rho_{i}, I} \supset U_{\rho_{i}, \sigma_{i, i+1}, I} \cong U_{\sigma_{i, i+1}, I} \cong U_{\rho_{i+1}, \sigma_{i, i+1}, I} \subset U_{\rho_{i+1}, I}
$$

which generate an equivalence relation on $\coprod_{i} U_{\rho_{i}, I}$, and the quotient by this equivalence relation defines a scheme $X_{I}^{o}$ over $\operatorname{Spec} R_{I}$.

One checks easily that the canonical isomorphisms of

$$
U_{\rho_{i}, \sigma_{i, i+1}, I} \subseteq U_{\rho_{i}, I} \text { and } U_{\rho_{i+1}, \sigma_{i, i+1}, I} \subseteq U_{\rho_{i+1}, I}
$$

satisfy the requirements for gluing data for schemes along open subsets, see e.g., [H77], Ex. II 2.12.

Remark 2.8. $X_{I}^{o}$ only depends on the equivalence class of $\varphi$, since the monoids $P_{\varphi_{\tau}}$ are canonically defined, independently of the choice of representative for $\varphi$.

We first analyze this construction in the purely toric case:

Lemma 2.9. For $(Y, D)$ toric and $\varphi$ a single-valued convex function on $B=M_{\mathbb{R}}, X_{I}^{o}$ is an open subscheme of the Mumford degeneration $\operatorname{Spec} \mathbb{k}\left[P_{\varphi}\right] / I \mathbb{k}\left[P_{\varphi}\right]$, and

$$
H^{0}\left(X_{I}^{o}, \mathcal{O}_{X_{I}^{o}}\right)=\mathbb{k}\left[P_{\varphi}\right] / I \mathbb{k}\left[P_{\varphi}\right] .
$$

Proof. Note that for $\tau \in \Sigma, \tau \neq\{0\}$, the monoid $P_{\varphi_{\tau}}$ is isomorphic to the localization of $P_{\varphi}$ along the face $\{(m, \varphi(m)) \mid m \in \tau \cap M\}$. Thus $\operatorname{Spec} \mathbb{k}\left[P_{\varphi_{\tau}}\right]$ is an open subset of $\operatorname{Spec} \mathbb{k}\left[P_{\varphi}\right]$ and $\operatorname{Spec} \mathbb{k}\left[P_{\varphi_{\tau}}\right] \otimes_{\mathbb{k}[P]} \mathbb{k}[P] / I$ is an open subset of Spec $\mathbb{k}\left[P_{\varphi}\right] / I \mathbb{k}\left[P_{\varphi}\right]$. Furthermore, the gluing procedure constructing $X_{I}^{o}$ is clearly compatible with these inclusions, so $X_{I}^{o}$ is an open subscheme of $\operatorname{Spec} \mathbb{k}\left[P_{\varphi}\right] / I \mathbb{k}\left[P_{\varphi}\right]$. Next, looking at the fibre over a closed point, one sees easily that the underlying topological space of these fibres is obtained just by removing the zero-dimensional torus orbit from the corresponding fibre of the Mumford degeneration. The closed fibres of the Mumford degeneration are $S_{2}$ by [02, 2.3.19. Thus by Lemma 2.10, the result follows.

Lemma 2.10. Let $\pi: \mathcal{X} \rightarrow S$ be a flat family of surfaces such that the fibre $\mathcal{X}_{s}$ satisfies Serre's condition $S_{2}$ for each $s \in S$. Let $i: \mathcal{X}^{o} \subset \mathcal{X}$ be the inclusion of an open subset such that the complement has finite fibres. Then $i_{*} \mathcal{O}_{\mathcal{X}^{o}}=\mathcal{O}_{\mathcal{X}}$. Similarly, if $\mathcal{F}$ is a coherent sheaf on $S$ then $i_{*}\left(\mathcal{O}_{\mathcal{X}^{0}} \otimes \pi^{*} \mathcal{F}\right)=\mathcal{O}_{\mathcal{X}} \otimes \pi^{*} \mathcal{F}$.

Proof. For the first statement see, e.g., [H04, Lemma A.3, (the assumption that the fibres are semi log canonical is not used). The second statement follows from the first by dévissage. 
Definition 2.11. Let $B_{0}(\mathbb{Z})$ denote the set of points of $B_{0}$ with integral coordinates in an integral affine chart. We also write $B(\mathbb{Z})=B_{0}(\mathbb{Z}) \cup\{0\}$.

Given the description of Remark 2.6, the following lemma is obvious.

Lemma 2.12. Suppose $n \geq 3$ and we are given a convex multivalued piecewise linear function $\varphi$ and a radical monomial ideal $J \subset P$ such that $\kappa_{\rho, \varphi} \in J$ for all rays $\rho \in$ $\Sigma$. Then if $x \in \operatorname{Spec} R_{J}$ is a closed point, the fibre of $X_{J}^{o} \rightarrow \operatorname{Spec} R_{J}$ over $x$ is $($ Spec $\mathbb{k}[\Sigma]) \backslash\{0\}$. Here, $\mathbb{k}[\Sigma]$ denotes the $\mathbb{k}$-algebra with a $\mathbb{k}$-basis $\left\{z^{m} \mid m \in B(\mathbb{Z})\right\}$ with multiplication given exactly as in (1.6), and 0 is the closed point whose ideal is generated by $\left\{z^{m} \mid m \neq 0\right\}$. In particular, the fibre is isomorphic to $\mathbb{V}_{n}^{o}$. Furthermore, with $R_{J}[\Sigma]:=R_{J} \otimes_{\mathbb{k}} \mathbb{k}[\Sigma]$,

$$
X_{J}^{o} \cong\left(\operatorname{Spec} R_{J}[\Sigma]\right) \backslash\left(\operatorname{Spec} R_{J}\right) \times\{0\}
$$

2.2. Scattering diagrams on $B$. Next we translate into algebraic geometry the instanton corrections. To construct our mirror family we will use the canonical scattering diagram $\mathfrak{D}^{\text {can }}$ defined in $\oiint 3.1$, (which is the translation of the instanton corrections associated to Maslov index zero disks), but as the regluing process works for any scattering diagram (and we will make use of this greater generality in [K3]), we carry it out for an arbitrary scattering diagram.

We continue with the notation of the previous sections, with $(Y, D),(B, \Sigma), P$ an arbitrary toric monoid, and $\varphi$ given. We also fix a monomial ideal $J \subset P$ such that $J=\sqrt{J}$. Denote by $\widehat{R}$ the completion of $\mathbb{k}[P]$ with respect to the ideal $J$, and for any $\tau \in \Sigma, \tau \neq 0$, denote by

$$
\widehat{\mathbb{k}\left[P_{\varphi_{\tau}}\right]}
$$

the completion of the ring $\mathbb{k}\left[P_{\varphi_{\tau}}\right]$ with respect to the ideal $J \mathbb{k}\left[P_{\varphi_{\tau}}\right]$.

We will now define a scattering diagram, which encodes a modification of the construction of $X_{I}^{o}$. Unlike the previous subsection, where we assumed $n \geq 3$ for ease of exposition throughout, in this subsection we can allow any number of irreducible components of $D$ except where noted.

Definition 2.13. A scattering diagram for the data $(B, \Sigma), P, \varphi$, and $J$ is a set

$$
\mathfrak{D}=\left\{\left(\mathfrak{d}, f_{\mathfrak{d}}\right)\right\}
$$

where

(1) $\mathfrak{d} \subset B$ is a ray in $B$ with endpoint the origin with rational slope. $\mathfrak{d}$ may coincide with a ray of $\Sigma$, or lie in the interior of a two-dimensional cone of $\Sigma$. 
(2) Let $\tau_{\mathfrak{d}} \in \Sigma$ be the smallest cone containing $\mathfrak{d}$. Then $f_{\mathfrak{d}}$ is a formal sum

$$
f_{\mathfrak{d}}=1+\sum_{p} c_{p} z^{p} \in \widehat{\mathbb{k}\left[P_{\varphi_{\tau_{\mathfrak{d}}}}\right]}
$$

for $c_{p} \in \mathbb{k}$ and $p$ running over elements of $P_{\varphi_{\tau_{\mathfrak{d}}}}$ such that $r(p) \neq 0$ and $r(p)$ is tangent to $\mathfrak{d}$. Here $r$ is defined by (2.1). We further require that $\mathfrak{d}$ satisfy one of the following two properties:

(a) For those $p$ with $c_{p} \neq 0, r(p)$, viewed as a tangent vector at an interior point of $\mathfrak{d}$, points towards the origin, in which case we say that $\mathfrak{d}$ is an outgoing ray.

(b) For those $p$ with $c_{p} \neq 0, r(p)$ points away from the origin, in which case we say that $\mathfrak{d}$ is an incoming ray.

(3) If $\operatorname{dim} \tau_{\mathfrak{d}}=2$ or if $\operatorname{dim} \tau_{\mathfrak{d}}=1$ and $\kappa_{\tau_{\mathfrak{d}}, \varphi} \notin J$, then $f_{\mathfrak{d}} \equiv 1 \bmod J$.

(4) For any ideal $I \subset P$ with $\sqrt{I}=J$, there are only a finite number of $\left(\mathfrak{d}, f_{\mathfrak{o}}\right) \in \mathfrak{D}$ such that $f_{\mathfrak{d}} \not \equiv 1 \bmod I \mathbb{k}\left[P_{\varphi_{\tau_{\mathfrak{d}}}}\right]$.

Construction 2.14. We now explain how a scattering diagram $\mathfrak{D}$ is used to modify the construction of $X_{I}^{o}$, as given in Construction 2.7, Suppose we are given a scattering diagram $\mathfrak{D}$ for the data $(B, \Sigma), P, \varphi$ and $J$, and an ideal $I$ with $\sqrt{I}=J$. We assume that $\kappa_{\rho, \varphi} \in J$ for all rays $\rho \in \Sigma$ and that $n \geq 3$ as in Construction 2.7 .

We will use the scattering diagram $\mathfrak{D}$ to modify both the definition of the rings $R_{\rho_{i}, I}$ as well as the gluings of the schemes defined by these rings. First, we modify the definition of $R_{\rho_{i}, I}$, setting

$$
R_{\rho_{i}, I}:=\frac{R_{I}\left[X_{i-1}, X_{i}^{ \pm 1}, X_{i+1}\right]}{\left(X_{i-1} X_{i+1}-z^{\kappa_{\rho_{i}, \varphi}} X_{i}^{-D_{\rho_{i}}^{2}} f_{\rho_{i}}\right)}
$$

Here $f_{\rho_{i}}$ is an element of $R_{I}\left[X_{i}^{ \pm 1}\right]$ defined by

$$
f_{\rho_{i}}=\prod_{\substack{\left(\mathfrak{d}, f_{\mathfrak{f}}\right) \in \mathcal{D} \\ \mathfrak{d}=\rho_{i}}} f_{\mathfrak{d}} \bmod I \mathbb{K}\left[P_{\varphi_{\rho_{i}}}\right]
$$

identifying $X_{i}$ with $z^{\varphi_{i}}\left(v_{i}\right)$ as in Proposition 2.5. Note this is a generalization of the old definition of $R_{\rho_{i}, I}$, which we obtain if $f_{\rho_{i}}=1$. Thus we continue to use the same notation.

Retaining the definition $R_{\sigma, I}=\mathbb{k}\left[P_{\varphi_{\sigma}}\right] \otimes_{R} R_{I}$ for $\operatorname{dim} \sigma=2$ from the previous subsection, we note that there are maps

$$
\psi_{\rho_{i},-}: R_{\rho_{i}, I} \rightarrow R_{\sigma_{i-1, i}, I}, \quad \psi_{\rho_{i},+}: R_{\rho_{i}, I} \rightarrow R_{\sigma_{i, i+1}, I}
$$


given by

$$
\begin{aligned}
& \psi_{\rho_{i},-}\left(X_{i}\right)=z^{\varphi_{\rho_{i}}\left(v_{i}\right)}, \quad \psi_{\rho_{i},-}\left(X_{i-1}\right)=z^{\varphi_{\rho_{i}}\left(v_{i-1}\right)}, \quad \psi_{\rho_{i},-}\left(X_{i+1}\right)=f_{\rho_{i}} z^{\varphi_{\rho_{i}}\left(v_{i+1}\right)}, \\
& \psi_{\rho_{i},+}\left(X_{i}\right)=z^{\varphi_{\rho_{i}}\left(v_{i}\right)}, \quad \psi_{\rho_{i},+}\left(X_{i-1}\right)=f_{\rho_{i}} z^{\varphi_{\rho_{i}}\left(v_{i-1}\right)}, \quad \psi_{\rho_{i},+}\left(X_{i+1}\right)=z^{\varphi_{\rho_{i}}\left(v_{i+1}\right)} .
\end{aligned}
$$

Furthermore, $\psi_{\rho_{i}, \pm}$ induce isomorphisms

$$
\psi_{\rho_{i},+}:\left(R_{\rho_{i}, I}\right)_{X_{i+1}} \rightarrow R_{\sigma_{i, i+1}, I}, \quad \psi_{\rho_{i},-}:\left(R_{\rho_{i}, I}\right)_{X_{i-1}} \rightarrow R_{\sigma_{i-1, i}, I}
$$

Set for $\tau \in \Sigma \backslash\{0\}$

$$
U_{\tau, I}:=\operatorname{Spec} R_{\tau, I}
$$

One checks easily that the natural map $U_{\rho, I} \rightarrow \operatorname{Spec} R_{I}$ is flat. The maps $\psi_{\rho_{i}, \pm}$ induces canonical embeddings $U_{\sigma_{i-1, i}, I}, U_{\sigma_{i, i+1}, I} \hookrightarrow U_{\rho_{i}, I}$, and we denote their image by $U_{\rho_{i}, \sigma_{i-1, i}, I}$ and $U_{\rho_{i}, \sigma_{i, i+1}, I}$ respectively. Note that (2.6) continues to hold.

Next, consider $\left(\mathfrak{d}, f_{\mathfrak{d}}\right) \in \mathfrak{D}$ with $\tau_{\mathfrak{o}}=\sigma \in \Sigma_{\max }$. Let $\gamma$ be a path in $B_{0}$ which crosses $\mathfrak{d}$ transversally at time $t_{0}$. Then define

$$
\theta_{\gamma, \mathfrak{d}}: R_{\sigma, I} \rightarrow R_{\sigma, I}
$$

by

$$
\theta_{\gamma, \mathfrak{d}}\left(z^{p}\right)=z^{p} f_{\mathfrak{d}}^{\left\langle n_{\mathfrak{d}}, r(p)\right\rangle}
$$

where $n_{\mathfrak{d}} \in \Lambda_{\sigma}^{*}$ is primitive and satisfies, with $m$ a non-zero tangent vector of $\mathfrak{d}$,

$$
\left\langle n_{\mathfrak{o}}, m\right\rangle=0, \quad\left\langle n_{\mathfrak{d}}, \gamma^{\prime}\left(t_{0}\right)\right\rangle<0 .
$$

If $\gamma$ is not differentiable at $t_{0}$, which might occur for broken lines, see Definition 2.16, this inequality is interpreted to mean that $n_{\mathfrak{d}}$ is positive at $\gamma\left(t_{0}-\epsilon\right)$ and negative at $\gamma\left(t_{0}+\epsilon\right)$ for $\epsilon>0$ small. Note that $f_{\mathfrak{o}}$ is invertible in $R_{\sigma, I}$ since $f_{\mathfrak{d}} \equiv 1 \bmod J \mathbb{k}\left[P_{\varphi_{\sigma}}\right]$, so $f_{\mathfrak{d}}-1$ is nilpotent in $R_{\sigma, I}$.

Let $\mathfrak{D}_{I} \subset \mathfrak{D}$ be the finite set of rays $\left(\mathfrak{d}, f_{\mathfrak{d}}\right)$ with $f_{\mathfrak{d}} \not \equiv 1 \bmod I \mathbb{k}\left[P_{\varphi_{\tau_{\mathfrak{d}}}}\right]$. For a path $\gamma$ wholly contained in the interior of $\sigma \in \Sigma_{\max }$ and crossing elements of $\mathfrak{D}_{I}$ transversally, we define

$$
\theta_{\gamma, \mathfrak{D}}:=\theta_{\gamma, \mathfrak{d}_{n}} \circ \cdots \circ \theta_{\gamma, \mathfrak{d}_{1}}
$$

where $\gamma$ crosses precisely the elements $\left(\mathfrak{d}_{1}, f_{\mathfrak{d}_{1}}\right), \ldots,\left(\mathfrak{d}_{n}, f_{\mathfrak{d}_{n}}\right)$ of $\mathfrak{D}_{I}$, in the given order. Note that if two rays $\mathfrak{d}_{i}, \mathfrak{d}_{i+1}$ in fact coincide as subsets of $B$, then $\theta_{\gamma, \mathfrak{d}_{i}}$ and $\theta_{\gamma, \mathfrak{d}_{i+1}}$ commute, so the ordering is not important for overlapping rays.

To construct $X_{I, \mathfrak{D}}^{o}$, we modify the gluings of the sets $U_{\rho, I}$ along the open subsets $U_{\rho, \sigma, I}$. For each $i$, we have canonical identifications of open subsets

$$
U_{\rho_{i}, I} \supset U_{\rho_{i}, \sigma_{i, i+1}, I} \cong U_{\sigma_{i, i+1}, I} \cong U_{\rho_{i+1}, \sigma_{i, i+1}, I} \subset U_{\rho_{i+1}, I}
$$

We can modify this identification via any automorphism of $U_{\sigma_{i, i+1}}$. We do this by choosing a path $\gamma_{i}:[0,1] \rightarrow B$ whose image is contained in the interior of $\sigma_{i, i+1}$, with 


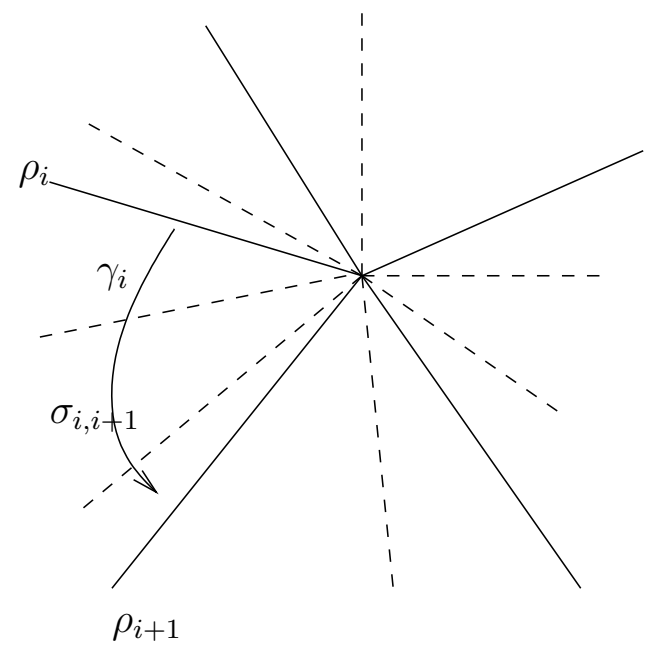

Figure 2.1. The path $\gamma_{i}$. The solid lines indicate the fan, the dotted lines are additional rays in $\mathfrak{D}$. The solid lines may also support rays in $\mathfrak{D}$

$\gamma_{i}(0)$ a point in $\sigma_{i, i+1}$ close to $\rho_{i}$ and $\gamma_{i}(1) \in \sigma_{i, i+1}$ close to $\rho_{i+1}$, chosen so that $\gamma_{i}$ crosses every ray $\left(\mathfrak{d}, f_{\mathfrak{d}}\right)$ of $\mathfrak{D}_{I}$ with $\tau_{\mathfrak{d}}=\sigma_{i, i+1}$ exactly once, see Figure 2.1.

We then obtain an automorphism

$$
\theta_{\gamma_{i}, \mathfrak{D}}: R_{\sigma_{i, i+1}, I} \rightarrow R_{\sigma_{i, i+1}, I}
$$

hence, after taking Spec, an isomorphism

$$
\theta_{\gamma_{i}, \mathfrak{D}}: U_{\rho_{i+1}, \sigma_{i, i+1}, I} \rightarrow U_{\rho_{i}, \sigma_{i, i+1}, I}
$$

We now define $X_{I, \mathfrak{D}}^{o}$ by dividing out $\coprod_{i} U_{\rho_{i}, I}$ by the equivalence relation given by identifying $x \in U_{\rho_{i+1}, \sigma_{i, i+1}, I} \subseteq U_{\rho_{i+1}, I}$ with $\theta_{\gamma_{i}, \mathfrak{D}}(x) \in U_{\rho_{i}, \sigma_{i, i+1}, I} \subseteq U_{\rho_{i}, I}$.

2.3. Broken lines. We continue to fix a rational surface with anti-canonical cycle $(Y, D)$ as usual, $D$ having an arbitrary number of irreducible components, giving $(B, \Sigma)$, as well as a monoid $P$, a multivalued $P$-convex function $\varphi$ on $B, J \subset P$ an ideal with $\sqrt{J}=J$, and a scattering diagram $\mathfrak{D}$ for this data. Broken lines were introduced in [G09] and their theory was further developed in [CPS].

Definition 2.15. Let $B$ be an integral affine manifold. An integral affine map $\gamma$ : $\left(t_{1}, t_{2}\right) \rightarrow B$ from an open interval $\left(t_{1}, t_{2}\right)$ is a continuous map such that for any integral affine coordinate chart $\psi: U \rightarrow \mathbb{R}^{n}$ of $B, \psi \circ \gamma: \gamma^{-1}(U) \rightarrow \mathbb{R}^{n}$ is integral affine, i.e., is given by $t \mapsto t v+b$ for some $v \in \mathbb{Z}^{n}$ and $b \in \mathbb{R}^{n}$.

Note that for an integral affine map, $\gamma^{\prime}(t) \in \Lambda_{B, \gamma(t)}$.

Definition 2.16. A broken line $\gamma$ in $(B, \Sigma)$ for $q \in B_{0}(\mathbb{Z})$ with endpoint $Q \in B_{0}$ is a proper continuous piecewise integral affine map $\gamma:(-\infty, 0] \rightarrow B_{0}$ with only a 
finite number of domains of linearity, together with, for each $L \subset(-\infty, 0]$ a maximal connected domain of linearity of $\gamma$, a choice of monomial $m_{L}=c_{L} z^{q_{L}}$ where $c_{L} \in \mathbb{k}^{\times}$ and $q_{L} \in \Gamma\left(L,\left.\gamma^{-1}(\mathcal{P})\right|_{L}\right)$, satisfying the following properties.

(1) For the unique unbounded domain of linearity $L,\left.\gamma\right|_{L}$ goes off to infinity in a cone $\sigma \in \Sigma_{\max }$ as $t \rightarrow-\infty$, and $q \in \sigma$. Furthermore, using the identification of the stalk $\mathcal{P}_{x}$ for $x \in \sigma$ with $\mathcal{P}_{\sigma}, m_{L}=z^{\varphi_{\sigma}(q)}$.

(2) For each $L$ and $t \in L,-r\left(q_{L}\right)=\gamma^{\prime}(t)$, where $r$ is defined in (2.1). Also $\gamma(0)=Q \in B_{0}$.

(3) Let $t \in(-\infty, 0)$ be a point at which $\gamma$ is not linear, passing from domain of linearity $L$ to $L^{\prime}$. If $\gamma(t) \in \tau \in \Sigma$, then $\mathcal{P}_{\gamma(t)}=\mathcal{P}_{\tau}$, so that we can view $q_{L} \in \mathcal{P}_{\tau}$ and $r\left(q_{L}\right) \in \Lambda_{\tau}$. Let $\mathfrak{d}_{1}, \ldots, \mathfrak{d}_{p} \in \mathfrak{D}$ be the rays of $\mathfrak{D}$ that contain $\gamma(t)$, with attached functions $f_{\mathfrak{d}_{j}}$. Then we require that $\gamma$ passes from one side of these rays to the other at time $t$, so that $\theta_{\gamma, \mathfrak{d}_{j}}$ is defined. Let $n=n_{\mathfrak{d}_{j}}$ be the primitive element of $\Lambda_{\tau}^{*}$ used to define $\theta_{\gamma, \mathfrak{d}_{j}}$. Expand

$$
\prod_{j=1}^{p} f_{\mathfrak{d}_{j}}^{\left\langle n, r\left(q_{L}\right)\right\rangle}
$$

as a formal power series in $\widehat{\mathbb{k}\left[P_{\varphi_{\tau}}\right]}$. Then there is a term $c z^{s}$ in this sum with

$$
m_{L^{\prime}}=m_{L} \cdot\left(c z^{s}\right) \text {. }
$$

Remark 2.17. Using the notation of item (3) above, by item (2) of the definition,

$$
\left\langle n, r\left(q_{L}\right)\right\rangle>0 \text {. }
$$

This is vital to interpret (2.9). Indeed, if $\tau$ is a ray, $f_{\mathfrak{d}_{i}}$ need not be invertible in $\widehat{\mathbb{k}\left[P_{\varphi_{\tau}}\right]}$, so (2.10) tells us that (2.9) makes sense in this ring.

Example 2.18. We give a first example of broken lines, in the case where $B$ is as given in Example 1.10 and $\mathfrak{D}=\emptyset$, so that there is no possibility of bending. Nevertheless, there is quite non-trivial behaviour. For an example including bending, see Example 3.7 after the introduction of the canonical scattering diagram.

Given $q \in B_{0}(\mathbb{Z}), Q \in B_{0}$ general, we can choose lifts $\tilde{q}, \tilde{Q}$ to the universal cover $\tilde{B}_{0}=|\tilde{\Sigma}| \backslash\{0\}$ of $B_{0}$. Let $\pi: \tilde{B}_{0} \rightarrow B_{0}$ be the covering map. Fixing the lift $\tilde{Q}$, for any lift $\tilde{q}$ we obtain a broken line $\gamma:(-\infty, 0] \rightarrow B_{0}$ given by $\gamma(t)=\pi(\tilde{Q}-t \tilde{q})$. As this has one domain of linearity $L$, we decorate $L$ with the monomial $z^{\varphi_{\sigma}(q)}$, where $q \in \sigma \in \Sigma$. Note there are an infinite number of such broken lines, one for each lift of $q$. Dealing with this non-finiteness is a key part of the proof of Looijenga's conjecture in $\$ 7$.

The next lemma and corollary are crucial for interpreting the monomials $m_{L}$ : 
Lemma 2.19. Let $\sigma_{-}, \sigma_{+} \in \Sigma_{\max }$ be the two maximal cones containing the ray $\rho \in \Sigma$. If $q \in P_{\varphi_{\sigma_{-}}}$with $-r(q) \in \operatorname{Int}\left(\rho^{-1} \sigma_{+}\right) \subset \Lambda_{\rho} \otimes_{\mathbb{Z}} \mathbb{R}$, then

$$
q \in P_{\varphi_{\rho}}=P_{\varphi_{\sigma_{-}}} \cap P_{\varphi_{\sigma_{+}}} .
$$

Proof. By the definitions there exist $p, \kappa_{\rho, \varphi} \in P$ and $n_{\rho} \in \Lambda_{\rho}^{*}$ annihilating the tangent space to $\rho$ and positive on $\sigma_{+}$such that

$$
\begin{aligned}
q & =\varphi_{\sigma_{-}}(r(q))+p \\
\varphi_{\sigma_{+}}(-r(q)) & =\varphi_{\sigma_{-}}(-r(q))+\left\langle n_{\rho},-r(q)\right\rangle \kappa_{\rho, \varphi} .
\end{aligned}
$$

Since $\left\langle n_{\rho},-r(q)\right\rangle>0$

$$
q=\varphi_{\sigma_{+}}(r(q))+p+\left\langle n_{\rho},-r(q)\right\rangle \kappa_{\rho, \varphi} \in P_{\varphi_{\sigma_{+}}} .
$$

An immediate consequence of this lemma is

Corollary 2.20. (1) Let $\gamma:\left[t_{1}, t_{2}\right] \rightarrow B_{0}$ be integral affine. Suppose that $\gamma\left(t_{1}\right) \in$ $\tau_{1}, \gamma\left(t_{2}\right) \in \tau_{2}$. Suppose also we are given a section $q \in \Gamma\left(\gamma^{-1} \mathcal{P}\right)$ such that $-r(q)=\gamma^{\prime}(t)$ for each $t$. If

$$
q\left(t_{1}\right) \in P_{\varphi_{\tau_{1}}} \subset \mathcal{P}_{\tau_{1}}=\mathcal{P}_{\gamma\left(t_{1}\right)}
$$

then

$$
q\left(t_{2}\right) \in P_{\varphi_{\tau_{2}}} \subset \mathcal{P}_{\tau_{2}}=\mathcal{P}_{\gamma\left(t_{2}\right)}
$$

(2) If $\gamma$ is a broken line, $t \in L$ a maximal domain of linearity with $\gamma(t) \in \tau$, then

$$
q_{L} \in P_{\varphi_{\tau}} \subset \mathcal{P}_{\tau}=\mathcal{P}_{\gamma(t)}
$$

Proof. The first item follows immediately from the lemma. The second item follows from the fact that if $t \ll 0$ lies in the unbounded domain of linearity with $\gamma(t) \in \sigma$, then $m_{L}=z^{\varphi_{\tau}(q)} \in P_{\varphi_{\sigma}}$ by construction. Then this holds for all $t$ by item (1) and Definition 2.16, (3).

The convexity of $\varphi$ puts further restrictions on the monomial decorations of a broken line.

Definition 2.21. Let $J \subset P$ be a proper monoid ideal. For $p \in J$ there exists a maximal $k \geq 1$ such that $p=p_{1}+\cdots+p_{k}$ with $p_{i} \in J$. We define $\operatorname{ord}_{J}(p)$ to be this maximum, and define $\operatorname{ord}_{J}(p)=0$ if $p \in P \backslash J$.

For $x \in \tau, q \in P_{\varphi_{\tau}}$, define $\operatorname{ord}_{J, x}(q):=\operatorname{ord}_{J}\left(q-\varphi_{\tau}(r(q))\right)$. This measures how high $q$ is above the graph of $\varphi_{\tau}$. If $\gamma$ is a broken line and $t \in L$ a maximal domain of linearity, define

$$
\operatorname{ord}_{J, \gamma}(t)=\operatorname{ord}_{J, \gamma(t)}\left(q_{L}\right)
$$


using $\gamma(t) \in \tau$ and $q_{L} \in P_{\varphi_{\tau}} \subset \mathcal{P}_{\gamma(t)}$.

Lemma 2.22. Let $\gamma$ be a broken line. Then if $t<t^{\prime}$,

$$
\operatorname{ord}_{J, \gamma}(t) \leq \operatorname{ord}_{J, \gamma}\left(t^{\prime}\right)
$$

with strict inequality if either $t$ and $t^{\prime}$ lie in different domains of linearity or for some $t^{\prime \prime}$ with $t<t^{\prime \prime}<t^{\prime}, \gamma\left(t^{\prime \prime}\right)$ lies in a ray $\rho \in \Sigma$ with bending parameter $\kappa_{\rho, \varphi} \in J$.

Proof. This is immediate from the definitions and the proof of Lemma 2.19.

Definition 2.23. For $I$ an ideal in $P$ with $\sqrt{I}=J$, let

$$
\operatorname{Supp}_{I}(\mathfrak{D}):=\bigcup_{\mathfrak{d}} \mathfrak{d}
$$

where the union is over all $\left(\mathfrak{d}, f_{\mathfrak{d}}\right) \in \mathfrak{D}$ such that $f_{\mathfrak{d}} \not \equiv 1 \bmod I \mathbb{k}\left[P_{\varphi_{\tau_{\mathfrak{d}}}}\right]$. By Definition 2.13, (4), this is a finite union.

Definition 2.24. Let $I$ be an ideal of $P$ with $\sqrt{I}=J$, and let $Q \in B \backslash \operatorname{Supp}_{I}(\mathfrak{D})$, $Q \in \tau \in \Sigma$. For $q \in B_{0}(\mathbb{Z})$, define

$$
\operatorname{Lift}_{Q}(q):=\sum_{\gamma} \operatorname{Mono}(\gamma) \in \mathbb{k}\left[P_{\varphi_{\tau}}\right] / I \cdot \mathbb{k}\left[P_{\varphi_{\tau}}\right]
$$

where the sum is over all broken lines $\gamma$ for $q$ with endpoint $Q$, and $\operatorname{Mono}(\gamma)$ denotes the monomial attached to the last domain of linearity of $\gamma$. The word "Lift" is used to indicate that this is, as we shall show, a lifting of a monomial $z^{q}$ on $X_{J, \mathfrak{D}}^{o}$ to $X_{I, \mathfrak{D}}^{o}$. The fact that $\operatorname{Lift}_{Q}(q)$ lies in the stated ring follows from:

Lemma 2.25. Let $Q \in \sigma \in \Sigma_{\max }, q \in B_{0}(\mathbb{Z})$. Let $I$ be an ideal with $\sqrt{I}=J$. Assume that $\kappa_{\rho, \varphi} \in J$ for at least one ray $\rho \in \Sigma$. Then the following hold:

(1) The collection of $\gamma$ in Definition 2.24 with

$$
\operatorname{Mono}(\gamma) \notin I \cdot \mathbb{k}\left[P_{\varphi_{\sigma}}\right]
$$

is finite.

(2) If one boundary ray of the connected component of $B \backslash \operatorname{Supp}_{I}(\mathfrak{D})$ containing $Q$ is a ray $\rho \in \Sigma$, then $\operatorname{Mono}(\gamma) \in \mathbb{k}\left[P_{\varphi_{\rho}}\right]$, and the collection of $\gamma$ with

$$
\operatorname{Mono}(\gamma) \notin I \cdot \mathbb{k}\left[P_{\varphi_{\rho}}\right]
$$

is finite.

Proof. Note there is some $k$ such that $J^{k} \subset I$ because $\mathbb{k}[P]$ is Noetherian. If $\gamma$ is a broken line with $\operatorname{Mono}(\gamma) \notin I \cdot \mathbb{k}\left[P_{\varphi_{\sigma}}\right]$, then $\gamma$ crosses the rays of $\Sigma$ in a cyclic order. Indeed, this follows from condition (3) of Definition 2.16, as a broken line must cross from one side to the other of each ray of $\mathfrak{D}$ it intersects. From this, the hypotheses on 
the $\kappa_{\rho, \varphi}$ imply that in any set of at least $n$ consecutive rays of $\Sigma$ that it crosses, there is

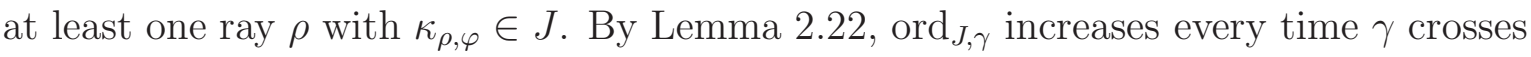
such a ray, and also every time $\gamma$ bends at a ray $\mathfrak{d}$ not contained in a ray of $\Sigma$. Once $\operatorname{ord}_{J, \gamma} \geq k, \operatorname{Mono}(\gamma) \in I \cdot \mathbb{k}\left[P_{\varphi_{\sigma}}\right]$. Hence there is an absolute bound on the number of rays of $\Sigma$ that $\gamma$ can cross, and the number of times $\gamma$ can bend. When $\gamma$ crosses a ray, there are a finite number of terms in (2.9) modulo $I \cdot \mathbb{k}\left[P_{\varphi_{\sigma}}\right]$, as the exponent is always positive, see Remark 2.17. Thus there are a finite number of possible choices of bend, and hence only a finite number of possible choices for the exponent of $\operatorname{Mono}(\gamma)$ modulo $I \cdot \mathbb{k}\left[P_{\varphi_{\sigma}}\right]$ once the initial monomial of $\gamma$ is fixed. Given any prescribed sequence of bends and initial direction, one sees that there is only one possibility for the underlying map $\gamma$ with endpoint a fixed point $Q$ by tracing the broken line back from $Q$. Each such underlying map $\gamma$ supports only a finite number of broken lines modulo $I \cdot \mathbb{k}\left[P_{\varphi_{\sigma}}\right]$. This yields the finiteness of (1).

The argument for the finiteness statement in (2), once the first part of (2) is established, is the same. For the first part of (2), consider a broken line $\gamma$ contributing to $\operatorname{Lift}_{Q}(q)$. We take $Q \in \sigma_{+}$, in the notation of Lemma 2.19. Write $\operatorname{Mono}(\gamma)=c_{L} z^{q_{L}}$. If $r\left(q_{L}\right) \in \rho^{-1} \sigma_{+}$then the statement follows from Lemma 2.19. Otherwise (by the definition of broken line) $\gamma$ crosses $\rho$, which is the last ray of $\Sigma$ and the last ray of $\operatorname{Supp}_{I}(\mathfrak{D})$ it crosses before reaching $Q$. Now the result follows from Lemma 2.19] and the definition of broken line.

Definition 2.26. Assume that $\kappa_{\rho, \varphi} \in J$ for at least one ray $\rho \in \Sigma$. We say a scattering diagram $\mathfrak{D}$ is consistent if for all ideals $I \subset P$ with $\sqrt{I}=J$ and for all $q \in B_{0}(\mathbb{Z})$, the following holds. Let $Q \in B_{0}$ be chosen so that the line joining the origin and $Q$ has irrational slope, and $Q^{\prime} \in B_{0}$ similarly. Then:

(1) If $Q, Q^{\prime} \in \sigma \in \Sigma_{\text {max }}$, then we can view $\operatorname{Lift}_{Q}(q)$ and $\operatorname{Lift}_{Q^{\prime}}(q)$ as elements of $R_{\sigma, I}$, and as such, we have

$$
\operatorname{Lift}_{Q^{\prime}}(q)=\theta_{\gamma, \mathfrak{D}}\left(\operatorname{Lift}_{Q}(q)\right)
$$

for $\gamma$ a path contained in the interior of $\sigma$ connecting $Q$ to $Q^{\prime}$.

(2) If $Q_{-} \in \sigma_{-}$and $Q_{+} \in \sigma_{+}$with $\sigma_{ \pm} \in \Sigma_{\max }$ and $\rho=\sigma_{+} \cap \sigma_{-}$a ray, and furthermore $Q_{-}$and $Q_{+}$are contained in connected components of $B \backslash \operatorname{Supp}_{I}(\mathfrak{D})$ whose closures contain $\rho$, then $\operatorname{Lift}_{Q_{ \pm}}(q) \in R_{\sigma_{ \pm}, I}$ are both images under $\psi_{\rho, \pm}$ of a single element

$$
\operatorname{Lift}_{\rho}(q) \in R_{\rho, I}
$$

Of course the definition is introduced so that the following construction works: 
Construction 2.27 (Construction of $\vartheta_{q}$ ). Suppose $D \subset Y$ has $n \geq 3$ irreducible components, and that $\mathfrak{D}$ is a consistent scattering diagram for data $(B, \Sigma), P, \varphi$ and $J$. Assume further that $\kappa_{\rho, \varphi} \in J$ for all $\rho \in \Sigma$, so that we may apply Construction 2.14. We now construct for any $I$ with $\sqrt{I}=J$ a function $\vartheta_{q} \in \Gamma\left(X_{I, \mathfrak{D}}^{o}, \mathcal{O}_{X_{I, \mathcal{Q}}^{o}}\right)$ for $q \in B(\mathbb{Z})=B_{0}(\mathbb{Z}) \cup\{0\}$.

We define $\vartheta_{0}=1$. Next, let $q \in B_{0}(\mathbb{Z})$. For each ray $\rho \in \Sigma$ contained in $\sigma_{ \pm} \in$ $\Sigma_{\max }$, choose two points $Q_{\rho}^{ \pm} \in B$, one each in the two connected components of $B \backslash$ $\left(\operatorname{Supp}_{I}(\mathfrak{D}) \cup \rho\right)$ which are adjacent to $\rho$, with $Q_{\rho}^{+} \in \sigma_{+}$and $Q_{\rho}^{-} \in \sigma_{-}$.

We first note that $\operatorname{Lift}_{Q_{\rho}^{ \pm}}(q)$ is a well-defined element of $R_{\sigma_{ \pm}, I}$, independent of the particular choice of $Q_{\rho}^{ \pm}$: given a choice say of $Q=Q_{\rho}^{+}$and another choice $Q^{\prime}$, we take a path $\gamma$ connecting $Q$ and $Q^{\prime}$ wholly contained in the connected component of $B \backslash\left(\operatorname{Supp}_{I}(\mathfrak{D}) \cup \rho\right)$ containing $Q$ and $Q^{\prime}$. By Definition 2.26, (1), it then follows that $\operatorname{Lift}_{Q}(q)=\operatorname{Lift}_{Q^{\prime}}(q)$.

By Definition 2.26, (2), we have an element $\operatorname{Lift}_{\rho}(q) \in R_{\rho, I}$ whose image under $\psi_{\rho, \pm}$ is $\operatorname{Lift}_{Q_{\rho}^{ \pm}}(q)$. It then follows via another application of Definition 2.26, (1), applied to the path of Figure 2.1, that if $\rho, \rho^{\prime}$ are adjacent rays in $\Sigma$, then $\operatorname{Lift}_{\rho}(q)$ and $\operatorname{Lift}_{\rho^{\prime}}(q)$ glue under the identification of open subsets of $U_{\rho, I}$ and $U_{\rho^{\prime}, I}$ given by $\theta_{\gamma, \mathfrak{D}}$. Thus all these elements of the rings $R_{\rho, I}$ for $\rho \in \Sigma$ glue to give a regular function on $X_{I, \mathfrak{D}}^{o}$, by construction of this latter space. This regular function is what we call $\vartheta_{q}$.

Theorem 2.28. Suppose $D$ has $n \geq 3$ irreducible components, and let $\varphi$ be a multivalued piecewise linear function on $B$ such that $\kappa_{\rho, \varphi} \in J$ for all rays $\rho \in \Sigma$. Let $\mathfrak{D}$ be a consistent scattering diagram and $I \subset P$ an ideal with $\sqrt{I}=J$. Set

$$
X_{I}:=\operatorname{Spec} \Gamma\left(X_{I, \mathfrak{P}}^{o}, \mathcal{O}_{X_{I, \mathfrak{Q}}^{o}}\right) .
$$

Since $X_{I, \mathfrak{D}}^{o}$ has the structure of a scheme over $\operatorname{Spec} R_{I}$, so does $X_{I}$, which we write as

$$
f_{I}: X_{I} \rightarrow \operatorname{Spec} R_{I}
$$

Then

(1) $X_{I}$ contains $X_{I, \mathfrak{D}}^{o}$ as an open subset and $f_{I}$ is flat with fibre over a closed point $x$ of $\operatorname{Spec} R_{I}$ isomorphic to the $n$-vertex $\mathbb{V}_{n}$.

(2) For each $q \in B(\mathbb{Z})$, there is a section $\vartheta_{q} \in \Gamma\left(X_{I}, \mathcal{O}_{X_{I}}\right)$, and the set

$$
\left\{\vartheta_{q} \mid q \in B(\mathbb{Z})\right\}
$$

is a free $R_{I}$-module basis for $\Gamma\left(X_{I}, \mathcal{O}_{X_{I}}\right)$.

Proof. Construction 2.27 constructs regular functions $\vartheta_{q}$ on $X_{I, \mathfrak{D}}^{o}$, hence by definition of $X_{I}$, we obtain $\vartheta_{q} \in \Gamma\left(X_{I}, \mathcal{O}_{X_{I}}\right)$. 
Now note that $X_{J, \mathfrak{D}}^{o}=X_{J}^{o}$ as defined in $\oiint 2.1$. Indeed, for any $\left(\mathfrak{d}, f_{\mathfrak{d}}\right) \in \mathfrak{D}$ with $\operatorname{dim} \tau_{\mathfrak{d}}=2$ we have $f_{\mathfrak{o}} \equiv 1 \bmod J$, so the open sets $U_{\rho_{i}, J}, U_{\rho_{i+1}, J}$ are glued trivially. Similarly, if $\operatorname{dim} \tau_{\mathfrak{o}}=1$ then since $\kappa_{\rho, \varphi} \in J$, the rings $R_{\rho, I}$ as given in (2.7) and (2.5) coincide and are glued trivially. Thus with $I=J$, we see the gluing constructions Constructions 2.7 and 2.14 coincide.

Note that with the assumption that $\kappa_{\rho, \varphi} \in J$ for all rays $\rho$,

$$
X_{J}^{o} \cong\left(\operatorname{Spec} R_{J}[\Sigma]\right) \backslash\left(\operatorname{Spec} R_{J}\right) \times\{0\}
$$

by Lemma 2.12, We see that the canonical map

$$
\bigoplus_{q \in B(\mathbb{Z})} R_{J} \cdot \vartheta_{q} \rightarrow \Gamma\left(X_{J}^{o}, \mathcal{O}_{X_{J}^{o}}\right)
$$

is an isomorphism. Indeed, by Lemma 2.10, $\Gamma\left(X_{J}^{o}, \mathcal{O}_{X_{J}^{o}}\right) \cong R_{J}[\Sigma]$. Furthermore, under this isomorphism, $\vartheta_{q}$ is clearly taken to $z^{q} \in R_{J}[\Sigma]$. This is because the only broken lines contributing to $\operatorname{Lift}_{Q}(q)$ modulo $J$ for any $Q$ is the straight line with endpoint $Q$, and this provides a contribution only if $Q$ lies in the same maximal cone as $q$.

It also follows that $X_{J}:=\operatorname{Spec} \Gamma\left(X_{J}^{o}, \mathcal{O}_{X_{J}^{o}}\right)=\operatorname{Spec} R_{J}[\Sigma]$ is flat over $\operatorname{Spec} R_{J}$ and the fiber over a closed point $x$ is given by $\operatorname{Spec} \mathbb{k}[\Sigma]$.

Now let $I$ be an ideal with $\sqrt{I}=J$. Let $i: X_{J}^{o} \subset X_{J}$ be the inclusion. Define a ringed space $X_{I}^{\prime}$ with underlying topological space $X_{J}$ by $\mathcal{O}_{X_{I}^{\prime}}:=i_{*} \mathcal{O}_{X_{I, \mathfrak{D}}^{o}}$. Then the natural map $\mathcal{O}_{X_{I}^{\prime}} \rightarrow \mathcal{O}_{X_{J}}$ is surjective by the existence of the lifts $\vartheta_{q}$. Thus $X_{I}^{\prime} / \operatorname{Spec} R_{I}$ is a flat deformation of $X_{J} / \operatorname{Spec} R_{J}$ by Lemma 2.29 below. Now since $X_{J}$ is affine it follows that $X_{I}^{\prime}$ is also affine, so $X_{I}^{\prime}=X_{I}:=\operatorname{Spec} \Gamma\left(X_{I, \mathfrak{D}}^{o}, \mathcal{O}_{X_{I, \mathfrak{D}}^{o}}\right)$.

We showed above that the $\vartheta_{q}$ form an $R_{J}$-module basis of $\Gamma\left(X_{J}, \mathcal{O}_{X_{J}}\right)$. Now since $X_{I} / \operatorname{Spec} R_{I}$ is a flat infinitesimal deformation of $X_{J} / \operatorname{Spec} R_{J}$ it follows that the $\vartheta_{q}$ form a $R_{I}$-module basis of $\Gamma\left(X_{I}, \mathcal{O}_{X_{I}}\right)$, see Lemma 2.30 below.

Lemma 2.29. Let $X_{0} / S_{0}$ be a flat family of surfaces such that the fibres satisfy Serre's condition $S_{2}$. Let $i: X_{0}^{o} \subset X_{0}$ be the inclusion of an open subset such that the complement has finite fibres. Note that $i_{*} \mathcal{O}_{X_{0}^{o}}=\mathcal{O}_{X_{0}}$ by Lemma 2.10 .

Let $S_{0} \subset S$ be an infinitesimal thickening of $S_{0}$ and let $X^{o} \rightarrow S$ be a flat deformation of $X_{0}^{o} / S_{0}$ over $S$. Define a family of ringed spaces $X \rightarrow S$ by $\mathcal{O}_{X}:=i_{*} \mathcal{O}_{X^{o}}$.

Then $X / S$ is a flat deformation of $X_{0} / S_{0}$ (that is, $X / S$ is flat and $X_{0}=X \times_{S} S_{0}$ ) if and only if the map

$$
\mathcal{O}_{X}:=i_{*} \mathcal{O}_{X^{o}} \rightarrow i_{*} \mathcal{O}_{X_{0}^{o}}=\mathcal{O}_{X_{0}}
$$

is surjective.

Proof. The condition is clearly necessary. 
Conversely, suppose (2.12) is surjective. Let $\mathcal{I} \subset \mathcal{O}_{S}$ be the nilpotent ideal defining $S_{0} \subset S$. Let $X_{n}^{o} / S_{n}$ denote the $n$th order infinitesimal thickening of $X_{0} / S_{0}$ determined by $X^{o} / S$, that is, $\mathcal{O}_{X_{n}^{o}}=\mathcal{O}_{X^{o}} / \mathcal{I}^{n+1} \cdot \mathcal{O}_{X^{o}}$ and $\mathcal{O}_{S_{n}}=\mathcal{O}_{S} / \mathcal{I}^{n+1}$. Define $X_{n} / S_{n}$ by $\mathcal{O}_{X_{n}}:=i_{*} \mathcal{O}_{X_{n}^{o}}$. Note that $\mathcal{O}_{X_{n}} \rightarrow \mathcal{O}_{X_{0}}$ is surjective because $\mathcal{O}_{X} \rightarrow \mathcal{O}_{X_{0}}$ is surjective by assumption. We show by induction on $n$ that $X_{n} / S_{n}$ is a flat deformation of $X_{0} / S_{0}$. For $n=0$ there is nothing to prove. Suppose the induction hypothesis is true for $n$. Since $X_{n+1}^{o} / S_{n+1}$ is flat (being the restriction of the flat family $X^{o} / S$ to $S_{n+1}$ ) we have a short exact sequence

$$
0 \rightarrow \mathcal{I}^{n+1} / \mathcal{I}^{n+2} \otimes \mathcal{O}_{X_{0}^{o}} \rightarrow \mathcal{O}_{X_{n+1}^{o}} \rightarrow \mathcal{O}_{X_{n}^{o}} \rightarrow 0
$$

Applying $i_{*}$ we obtain an exact sequence

$$
0 \rightarrow i_{*}\left(\mathcal{I}^{n+1} / \mathcal{I}^{n+2} \otimes \mathcal{O}_{X_{0}^{o}}\right) \rightarrow \mathcal{O}_{X_{n+1}} \rightarrow \mathcal{O}_{X_{n}}
$$

By Lemma 2.10 the first term is equal to $\mathcal{I}^{n+1} / \mathcal{I}^{n+2} \otimes \mathcal{O}_{X_{0}}$. Moreover, the last arrow is surjective because $\mathcal{O}_{X_{n+1}} \rightarrow \mathcal{O}_{X_{0}}$ is surjective, $\mathcal{O}_{X_{n}} / \mathcal{I} \cdot \mathcal{O}_{X_{n}}=\mathcal{O}_{X_{0}}$ by the induction hypothesis, and $\mathcal{I}$ is nilpotent, as in Theorem 8.4 of [Ma89] (where the module $M$ need not be finitely generated for the argument given there to work). So we have an exact sequence

$$
0 \rightarrow \mathcal{I}^{n+1} / \mathcal{I}^{n+2} \otimes \mathcal{O}_{X_{0}} \rightarrow \mathcal{O}_{X_{n+1}} \rightarrow \mathcal{O}_{X_{n}} \rightarrow 0
$$

It follows that $\mathcal{O}_{X_{n+1}} / \mathcal{I}^{n+1} \cdot \mathcal{O}_{X_{n+1}}=\mathcal{O}_{X_{n}}$. (Indeed, consider the map

$$
\alpha: \mathcal{I}^{n+1} \otimes \mathcal{O}_{X_{n+1}} \rightarrow \mathcal{O}_{X_{n+1}}, \quad \alpha(f \otimes g)=f g .
$$

We claim that $\alpha$ is equal to the composition of the map

$$
\beta: \mathcal{I}^{n+1} \otimes \mathcal{O}_{X_{n+1}} \rightarrow \mathcal{I}^{n+1} / \mathcal{I}^{n+2} \otimes \mathcal{O}_{X_{0}}
$$

given by the natural maps on the factors and the first map $\gamma$ of the exact sequence (2.13). Since $\mathcal{O}_{X_{n+1}}=i_{*} \mathcal{O}_{X_{n+1}^{o}}$ by definition, it suffices to check the equality after restriction to $X^{o}$, where it is obvious. The map $\beta$ is surjective because $\mathcal{O}_{X_{n+1}} \rightarrow \mathcal{O}_{X_{0}}$ is surjective. So the image of $\gamma$ is equal to the image of $\alpha$, namely $\mathcal{I}^{n+1} \cdot \mathcal{O}_{X_{n+1}}$.) Now by [Ma89, Theorem 22.3, p. 174, the exact sequence (2.13) shows that $X_{n+1} / S_{n+1}$ is a flat deformation of $X_{0} / S_{0}$.

Lemma 2.30. Let $A \rightarrow B$ be a flat homomorphism of Noetherian rings and $I \subset A a$ nilpotent ideal. Suppose given a set $S$ of elements of $B$ such that the reductions of the elements of $S$ form an $A / I$-module basis of $B / I B$. Then $S$ is an $A$-module basis of $B$.

Proof. Since $I$ is nilpotent and $S$ generates $B / I B$ it is clear that $S$ spans $B$ by Theorem 8.4 of [Ma89]. So we have an exact sequence

$$
0 \rightarrow K \rightarrow A^{S} \rightarrow B \rightarrow 0 .
$$


Tensoring with $A / I$ we obtain an exact sequence

$$
0 \rightarrow K / I K \rightarrow(A / I)^{S} \rightarrow B / I B \rightarrow 0
$$

using flatness of $B$ over $A$. We deduce that $K / I K=0$ by our assumption, hence $K=0$ because $I$ is nilpotent.

Proposition 2.31. Let $X_{I} / S_{I}:=\operatorname{Spec} R_{I}$ be the family of Theorem 2.28. Then the relative dualizing sheaf $\omega_{X_{I} / S_{I}}$ is trivial. It is generated by the global section $\Omega$ given on local patches $U_{\rho_{i}, I}$ by $\operatorname{dlog} X_{i-1} \wedge \operatorname{d} \log X_{i}=\operatorname{d} \log X_{i} \wedge d \log X_{i+1}$. Here we take the rays $\rho_{j}$ in counter-clockwise order, after choosing an orientation on $B$, to obtain a consistent choice of signs.

Proof. By the adjunction formula for the closed embedding

$$
U_{\rho_{i}} \subset \mathbb{A}_{X_{i-1}, X_{i+1}}^{2} \times \mathbb{G}_{m, X_{i}} \times S_{I}
$$

the dualizing sheaf $\omega_{X_{I} / S_{I}}$ is freely generated over $U_{\rho_{i}}$ by the local section in the statement. These sections patch to give a generator $\Omega$ of $\omega_{X_{I \mathscr{D}}^{o} / S_{I}}$ because the scattering automorphisms preserve the torus invariant two-forms. Both $\omega_{X_{I} / S_{I}}$ and $\mathcal{O}_{X_{I}}$ satisfy the relative $S_{2}$ property $i_{*} i^{*} \mathcal{F}=\mathcal{F}$ where $i: X_{I}^{o} \subset X_{I}$ is the inclusion ([H04], Appendix, where the hypothesis of slc is not needed), hence $\omega_{X_{I} / S_{I}}$ is freely generated by $\Omega$.

2.4. The algebra structure. In the previous section, we saw that the $R_{I}$-algebra

$$
A_{I}=\Gamma\left(X_{I, \mathfrak{D}}^{o}, \mathcal{O}_{X_{I, \mathfrak{D}}^{o}}\right)
$$

defining the flat deformation $X_{I}$ has an $R_{I}$-module basis of theta functions $\left\{\vartheta_{m} \mid m \in\right.$ $B(\mathbb{Z})$ \}. Here we derive a description of the multiplication rule on $R_{I}$ using the geometry of the integral affine manifold $B$. Besides being an attractive combinatorial description of the multiplication rule, we will use this (in the case of the canonical scattering diagram $\left.\mathfrak{D}^{\text {can }}\right)$ in $\sqrt[6]{6}$ to prove that our deformation extends over completions of larger strata of $\operatorname{Spec} \mathbb{k}[P]$, as well as for the case that $D$ has 1 or 2 irreducible components.

Definition 2.32. For a broken line $\gamma$ with endpoint $Q \in \tau \in \Sigma$, define $s(\gamma) \in \Lambda_{\tau}$, $c(\gamma) \in \mathbb{k}[P]$ by demanding that

$$
\operatorname{Mono}(\gamma)=c(\gamma) \cdot z^{\varphi_{\tau}(s(\gamma))}
$$

Write $\operatorname{Limits}(\gamma)=(q, Q)$ if $\gamma$ is a broken line for $q$ and has endpoint $Q \in B$.

Remark 2.33. Recall that $B_{0}$ in fact has the structure of an integral linear manifold. One feature of such manifolds is that for any simply connected set $U \subset B_{0}$, there is is a canonical linear immersion $U \rightarrow \Lambda_{\mathbb{R}, U}$, compatible with parallel transport inside $U$. 
In particular, if $q$ is a point of $B_{0}$ with $q \in \sigma \in \Sigma$, and $\tau \subset \sigma$, then the canonical embedding of a neighbourhood of $\tau \backslash\{0\}$ in $\Lambda_{\tau, \mathbb{R}}$ identifies $q$ with a point of $\Lambda_{\tau, \mathbb{R}}$.

Theorem 2.34. Let $q_{1}, q_{2} \in B(\mathbb{Z})$. In the canonical expansion

$$
\vartheta_{q_{1}} \cdot \vartheta_{q_{2}}=\sum_{q \in B(\mathbb{Z})} \alpha_{q} \vartheta_{q}
$$

where $\alpha_{q} \in R_{I}$ for each $q$, we have

$$
\alpha_{q}=\sum_{\substack{\left(\gamma_{1}, \gamma_{2}\right) \\ \operatorname{Limits}\left(\gamma_{i}\right)=\left(q_{i}, z\right) \\ s\left(\gamma_{1}\right)+s\left(\gamma_{2}\right)=q}} c\left(\gamma_{1}\right) c\left(\gamma_{2}\right)
$$

Here $z \in B_{0}$ is a point very close to $q$ contained in a cell $\tau$, and we identify $q$ with a point of $\Lambda_{\tau}$ using Remark 2.33.

Proof. To identify the coefficient of $\vartheta_{q}$, choose a point $z \in B$ very close to $q$, and describe the product using the lifts of $z^{q_{1}}, z^{q_{2}}$ at $z$ :

$$
\left(\operatorname{Lift}_{z}\left(q_{1}\right)\right)\left(\operatorname{Lift}_{z}\left(q_{2}\right)\right)=\sum_{q^{\prime}} \alpha_{q^{\prime}} \operatorname{Lift}_{z}\left(q^{\prime}\right) .
$$

Now observe first that there is only one broken line $\gamma$ with endpoint $z$ and $s(\gamma)=q \in$ $\Lambda_{\tau}$ : this is the broken line whose image is $z+\mathbb{R}_{\geq 0} q$. Indeed, the final segment of such a $\gamma$ is on this ray, and this ray meets no scattering rays, so the broken line cannot bend. Thus the coefficient of $\operatorname{Lift}_{z}(q)$ on the right-hand side of the above equation can be read off by looking at the coefficient (in $R_{I}$ ) of $z^{\varphi_{\tau}(q)}$. This gives the desired description.

\section{The CANonical SCATtering Diagram}

3.1. Definition. Here we give the precise definition of $\mathfrak{D}^{\text {can }}$. As explained in the introduction, it is, roughly speaking, defined in terms of maps $\mathbb{A}^{1} \rightarrow Y \backslash D$, which are algebro-geometric analogues of the holomorphic disks used for instanton corrections in the symplectic heuristic. We begin by recalling necessary facts about relative GromovWitten invariants used to count these curves.

Definition 3.1. Let $(\tilde{Y}, \tilde{D})$ be a non-singular rational surface with $\tilde{D}$ an anti-canonical cycle of rational curves, and let $C$ be an irreducible component of $\tilde{D}$. Consider a class $\beta \in A_{1}(\tilde{Y}, \mathbb{Z})$ such that

$$
\beta \cdot \tilde{D}_{i}= \begin{cases}k_{\beta} & \tilde{D}_{i}=C \\ 0 & \tilde{D}_{i} \neq C\end{cases}
$$


for some $k_{\beta}>0$. Let $F$ be the closure of $\tilde{D} \backslash C$, and let

$$
\tilde{Y}^{o}:=\tilde{Y} \backslash F, \quad C^{o}:=C \backslash F .
$$

Let $\overline{\mathfrak{M}}\left(\tilde{Y}^{o} / C^{o}, \beta\right)$ be the moduli space of stable relative maps of genus zero curves representing the class $\beta$ with tangency of order $k_{\beta}$ at an unspecified point of $C^{o}$. (See [Li00], Li02] for the algebraic definition for these relative Gromov-Witten invariants, and [LR01], [IP03] for the original symplectic definitions.) We refer to $\beta$ informally as an $\mathbb{A}^{1}$-class. The virtual dimension of this moduli space is

$$
-K_{\tilde{Y}} \cdot \beta+(\operatorname{dim} \tilde{Y}-3)-\left(k_{\beta}-1\right)=0 .
$$

Here the first two terms give the standard dimension formula for the moduli space of stable rational curves in $\tilde{Y}$ representing the class $\beta$, and the term $k_{\beta}-1$ is the change in dimension given by imposing the $k_{\beta}$-fold tangency condition. The moduli space carries a virtual fundamental class. Furthermore, we have

Lemma 3.2. $\overline{\mathfrak{M}}\left(\tilde{Y}^{o} / C^{o}, \beta\right)$ is proper over $\mathbb{k}$.

Proof. This follows as in the proof of [GPS09], Theorem 4.2. In brief, let $R$ be a valuation ring with quotient field $K$, with $S=\operatorname{Spec} R, T=\operatorname{Spec} K$. We would like to extend a morphism $T \rightarrow \overline{\mathfrak{M}}\left(\tilde{Y}^{o} / C^{o}, \beta\right)$ to $S$. We know that the moduli space $\overline{\mathfrak{M}}(\tilde{Y} / C, \beta)$ is proper, so we obtain a family of relative stable maps $\mathcal{C} \rightarrow S$ to $\tilde{Y}$. We just need to show that in fact the image of the closed fibre $\mathcal{C}_{0}$ lies in $\tilde{Y}^{o}$. However, the argument in the proof of GPS09, Theorem 4.2 shows that if the image of $\mathcal{C}_{0}$ intersects $F$, then $\mathcal{C}_{0}$ must be of genus at least 1 , which is not the case.

Given this, we define

$$
N_{\beta}:=\int_{\left[\overline{\mathfrak{M}}\left(\tilde{Y}^{o} / C^{o}, \beta\right)\right]^{v i r}} 1 .
$$

Morally, one should view $N_{\beta}$ as counting maps from affine lines to $\tilde{Y} \backslash \tilde{D}$ whose closures represent the class $\beta$.

In what follows, we fix as usual the pair $(Y, D)$, with tropicalisation $(B, \Sigma)$, and $\varphi$ the function given by Example 2.3 for some choice of $\eta: \mathrm{NE}(Y) \rightarrow P$. We can assume here that $D$ has an arbitrary number of irreducible components.

Definition 3.3. Fix a ray $\mathfrak{d} \subset B$ with endpoint the origin, with rational slope. If $\mathfrak{d}$ coincides with a ray of $\Sigma$, set $\Sigma^{\prime}:=\Sigma$; otherwise, let $\Sigma^{\prime}$ be a refinement of $\Sigma$ obtained by adding the ray $\mathfrak{d}$ and a number of other rays chosen so that each cone of $\Sigma^{\prime}$ is integral affine isomorphic to the first quadrant of $\mathbb{R}^{2}$. This gives a toric blow-up $\pi: \tilde{Y} \rightarrow Y$ (the identity in the first case) by Lemma 1.6. Let $C \subset \pi^{-1}(D)$ be the irreducible component corresponding to $\mathfrak{d}$. 
Let $\tau_{\mathfrak{d}} \in \Sigma$ be the smallest cone containing $\mathfrak{d}$. Let $m_{\mathfrak{d}} \in \Lambda_{\tau_{\mathfrak{d}}}$ be a primitive generator of the tangent space to $\mathfrak{d}$, pointing away from the origin. Define

$$
f_{\mathfrak{d}}:=\exp \left[\sum_{\beta} k_{\beta} N_{\beta} z^{\eta\left(\pi_{*}(\beta)\right)-\varphi_{\tau_{\mathfrak{d}}}\left(k_{\beta} m_{\mathfrak{o}}\right)}\right] .
$$

Here the sum is over all classes $\beta \in A_{1}(\tilde{Y}, \mathbb{Z})$ satisfying (3.1). Note that if $N_{\beta} \neq 0$, then necessarily $\overline{\mathfrak{M}}\left(\tilde{Y}^{o} / C^{o}, \beta\right)$ is non-empty, and thus $\beta \in \mathrm{NE}(\tilde{Y})$, so $\pi_{*}(\beta) \in \mathrm{NE}(Y)$. We note the numbers $N_{\beta}$ do not depend on the particular choice of refinement $\Sigma^{\prime}$. Indeed, further refining $\Sigma^{\prime}$ does not change the pair $\tilde{Y}^{o} / C^{o}$, and hence does not change the numbers $N_{\beta}$.

We define

$$
\mathfrak{D}^{\text {can }}:=\left\{\left(\mathfrak{d}, f_{\mathfrak{d}}\right) \mid \mathfrak{d} \subset B \text { a ray of rational slope }\right\} .
$$

We call a class $\beta \in A_{1}(\tilde{Y}, \mathbb{Z})$ an $\mathbb{A}^{1}$-class if $N_{\beta} \neq 0$.

Note that all rays of the canonical scattering diagram are outgoing.

Remark 3.4. In theory, one should be able to use logarithmic Gromov-Witten invariants ([GS11] or [AC11] ) to define $N_{\beta}$ without the technical trick of blowing up and working on an open variety. This would be done by working relative to $D$, and counting rational curves of class $\beta$ with one point mapping to the boundary with specified orders of tangency with each boundary divisor, with non-zero order of tangency with either one divisor $D_{i}$ or two adjacent divisors $D_{i}, D_{i+1}$. However, some additional arguments are required to compare logarithmic invariants with the invariants described above as developed in GPS09, and we do not wish to do this here.

Lemma 3.5. Let $J \subset P$ be an ideal with $\sqrt{J}=J$. Suppose the map $\eta: \mathrm{NE}(Y) \rightarrow P$ satisfies the following conditions:

(1) For any ray $\mathfrak{d} \subset B$ of rational slope, let $\pi: \tilde{Y} \rightarrow Y$ be the corresponding blowup. We require that if $\operatorname{dim} \tau_{\mathfrak{d}}=2$ or $\operatorname{dim} \tau_{\mathfrak{d}}=1$ and $\kappa_{\tau_{\mathfrak{d}}, \varphi} \notin J$ then for any $\mathbb{A}^{1}$-class $\beta$ contributing to $f_{\mathfrak{o}}$, we have $\eta\left(\pi_{*}(\beta)\right) \in J$.

(2) For any ideal $I$ with $\sqrt{I}=J$, there are only a finite number of $\mathfrak{d}$ and $\mathbb{A}^{1}$-classes $\beta$ such that $\eta\left(\pi_{*}(\beta)\right) \notin I$.

Then $\mathfrak{D}^{\text {can }}$ is a scattering diagram for the data $(B, \Sigma), P, \varphi$, and $J$.

Proof. Note that

$$
z^{\eta\left(\pi_{*}(\beta)\right)-\varphi_{\tau_{\mathfrak{d}}}\left(k_{\beta} m_{\mathfrak{\jmath}}\right)} \in I \mathbb{k}\left[P_{\varphi_{\tau_{\mathfrak{d}}}}\right]
$$

if and only if $\eta\left(\pi_{*}(\beta)\right) \in I$. So the hypotheses of the lemma imply conditions (2)-(4) of Definition 2.13, 
Example 3.6. Let $\sigma \subset A_{1}(Y) \otimes_{\mathbb{Z}} \mathbb{R}$ be a strictly convex rational polyhedral cone containing $\mathrm{NE}(Y)$. (This can be obtained as the dual of a strictly convex rational polyhedral cone in $\operatorname{Pic}(Y) \otimes_{\mathbb{Z}} \mathbb{R}$ which spans this latter space and is contained in the nef cone.) Let $P=\sigma \cap A_{1}(Y)$. Since $\sigma$ is strictly convex, $P^{\times}=0$. For any $\mathfrak{m}$ primary ideal $I, P \backslash I$ is a finite set. Let $\eta: \mathrm{NE}(Y) \rightarrow P$ be the inclusion. Then the finiteness hypotheses of the above Lemma hold for $J=\mathfrak{m}$ (note that the conditions (3.1) determine $\beta \in A_{1}(\tilde{Y})$ given $\left.\pi_{*}(\beta)\right)$.

Example 3.7. We return to the example $(Y, D)$ of a del Pezzo surface together with a cycle of $5(-1)$-curves studied in Example 1.9. Let $P=\mathrm{NE}(Y)$ and $\eta$ be the identity. Let $J=\mathfrak{m} \subset P$ and $I \subset P$ be an ideal with $\sqrt{I}=J$. Then $\mathfrak{D}^{\text {can }}$ consists of five rays:

$$
\mathfrak{D}^{\text {can }}=\left\{\left(\rho_{i}, 1+z^{\left[E_{i}\right]-\varphi_{\rho_{i}}\left(v_{i}\right)}\right) \mid 1 \leq i \leq 5\right\} .
$$

Here $E_{i}$ is the unique $(-1)$-curve in $Y$ which is not contained in $D$ and meets $D_{i}$ transversally, and $v_{i}$ is the primitive generator of the ray $\rho_{i}$ corresponding to $D_{i}$. To derive this formula from the above definition of the canonical scattering diagram one needs to show that the only possible stable relative maps contributing to $\mathfrak{D}^{\text {can }}$ are multiple covers of the $E_{i}$ 's, and that a $k$-fold multiple cover contributes a GromovWitten invariant of $(-1)^{k-1} / k^{2}$. It is easier to compute this using the main result of GPS09], which is done by way of Theorem 3.25. See Example 3.26.

If we accept this description of $\mathfrak{D}^{\text {can }}$, then we can describe all broken lines and the multiplication law given by this diagram.

We first note that no broken line can wrap around $0 \in B$, i.e., if a broken line leaves a cone $\sigma \in \Sigma_{\max }$, it will never return to that cone. It is enough to check this for a straight line (as the bending in any broken line for $\mathfrak{D}_{\text {can }}$ is always away from the origin), and this is easily verified, using e.g., Figure 1.1.

Next, since the only scattering rays are the rays $\rho \in \Sigma$, if $q, Q \in \sigma \in \Sigma_{\text {max }}$, then the obvious straight line is the unique broken line for $q$ with endpoint $Q$. Thus if we describe $\vartheta_{q}$ in the open subset of $X_{I, \mathfrak{D}^{\text {can }}}^{o}$ corresponding to $\sigma, \vartheta_{q}$ is just the monomial $z^{\varphi_{\sigma}(q)}$. It follows that

$$
\vartheta_{v_{i}}^{a} \vartheta_{v_{i+1}}^{b}=\vartheta_{a v_{i}+b v_{i+1}}
$$

for $a, b \geq 0$. In particular, the $\vartheta_{v_{i}}$ 's generate the $\mathbb{k}[P] / I$-algebra $\Gamma\left(X_{I}, \mathcal{O}_{X_{I}}\right)$, and the algebra structure is determined once we compute $\vartheta_{v_{i}} \cdot \vartheta_{v_{i+2}}$.

We consider a broken line for $v_{i}$. One checks the following, using Figure 1.1 and the above description of $\mathfrak{D}^{\text {can }}$ : The broken line can cross at most two rays of $\Sigma$, and it bends at most once, at the last ray of $\Sigma$ that it crosses. See Figure 3.1, ¿From this one deduces using Theorem 2.34:

$$
\vartheta_{v_{i-1}} \vartheta_{v_{i+1}}=z^{\left[D_{i}\right]}\left(\vartheta_{v_{i}}+z^{\left[E_{i}\right]}\right) .
$$




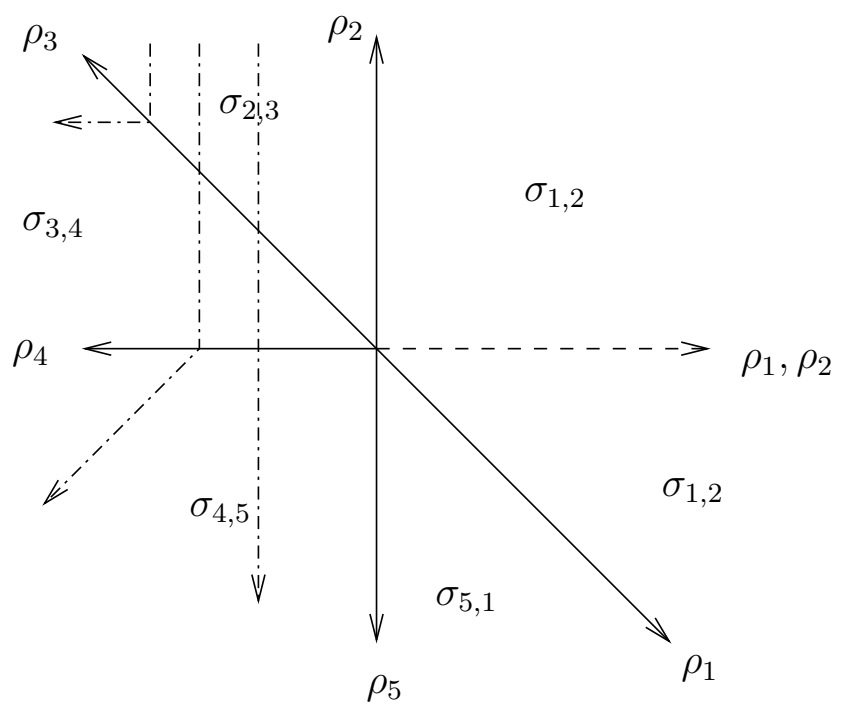

Figure 3.1. The different types of broken lines in Example 3.7.

The term $z^{\left[D_{i}\right]} \cdot \vartheta_{v_{i}}$ corresponds to two straight broken lines for $v_{i-1}, v_{i+1}$, with endpoint the point $v_{i}$ of $\rho_{i}$. The term $z^{\left[D_{i}\right]} \cdot z^{\left[E_{i}\right]}$ is the coefficient of $1=\vartheta_{0}$. To compute this we use the invariance of broken lines, and so choose a generic point $Q$ near 0 and compute the coefficient $\alpha_{0}$ of $\vartheta_{0}$ using pairs $\gamma_{i}$ as in Theorem 2.34 whose final directions are opposite, i.e., $s\left(\gamma_{1}\right)+s\left(\gamma_{2}\right)=0$. If we take $Q \in \sigma_{i, i+1}$, then there is exactly one term contributing to $\alpha_{0}: \gamma_{1}$ will bend once where it crosses $\rho_{i}$, and $\gamma_{2}$ is straight. Alternatively, one can use the explicit expressions for $\operatorname{Lift}_{Q}\left(v_{j}\right), j=i-1, i$ and $i+1$, and see they satisfy the relation (3.2).

One can check that the five equations (3.2) define $X_{I}$. These equations are algebraic, and in fact define a flat family over $\operatorname{Spec} \mathbb{k}[\mathrm{NE}(Y)]$. (This is always the case in the nonnegative semi-definite case, see Corollary 6.11).

Our goal now is to prove consistency of $\mathfrak{D}^{\text {can }}$, as stated in the following (the final step in the construction of our mirror family):

Theorem 3.8. Suppose that we are given a map $\eta: \mathrm{NE}(Y) \rightarrow P$ such that $\varphi$ is defined as in Example 2.3 by $\kappa_{\rho, \varphi}=\eta\left(\left[D_{\rho}\right]\right)$. Suppose furthermore the following conditions hold:

(I) For any $\mathbb{A}^{1}$-class $\beta, \eta\left(\pi_{*}(\beta)\right) \in J$;

(II) For any ideal I with $\sqrt{I}=J$, there are only a finite number of $\mathbb{A}^{1}$-classes $\beta$ such that $\eta\left(\pi_{*}(\beta)\right) \notin I$.

(III) $\eta\left(\left[D_{i}\right]\right) \in J$ for at least one boundary component $D_{i} \subset D$.

Then $\mathfrak{D}^{\text {can }}$ is a consistent scattering diagram. 
We include here an observation we will need later showing that the canonical scattering diagram only depends on the deformation class of $(Y, D)$.

Lemma 3.9. Let $(\mathcal{Y}, \mathcal{D}) \rightarrow S$ be a flat family of pairs over a connected base $S$, with each fibre $\left(\mathcal{Y}_{s}, \mathcal{D}_{s}\right)$ being a non-singular rational surface with anti-canonical cycle. Suppose further that there is a trivialization $\mathcal{D} \cong D \times S$ and the restriction map $\operatorname{Pic}(\mathcal{Y}) \rightarrow \operatorname{Pic}\left(\mathcal{Y}_{s}\right)$ is an isomorphism for any $s \in S$. This in particular gives a canonical identification $A_{1}\left(\mathcal{Y}_{s}, \mathbb{Z}\right)$ with $A_{1}\left(\mathcal{Y}_{s^{\prime}}, \mathbb{Z}\right)$ for any $s, s^{\prime} \in S$. Then for any $s, s^{\prime} \in S$, $\left(\mathcal{Y}_{s}, \mathcal{D}_{s}\right)$ and $\left(\mathcal{Y}_{s^{\prime}}, \mathcal{D}_{s^{\prime}}\right)$ induce the same canonical scattering diagram.

Proof. It is enough to show that the numbers $N_{\beta}$ are deformation invariants in the above sense, i.e., if we are given a family $\pi:(\tilde{\mathcal{Y}}, \tilde{\mathcal{D}}) \rightarrow S$ with each fibre as in Definition 3.1, with an irreducible component $\mathcal{C} \subset \tilde{\mathcal{D}}$, then the number

$$
N_{\beta, s}:=\int_{\left[\overline{\mathfrak{M}}_{\mathcal{Y}}\left(\tilde{\mathcal{Y}}_{s}^{o} / \mathcal{C}_{s}^{o}, \beta\right)\right]^{v i r}} 1
$$

is independent of $s$. Indeed, once this is shown, then if $N_{\beta, s} \neq 0$, necessarily $\beta$ defines a class in $\operatorname{NE}\left(\mathcal{Y}_{s}\right)$, as well as in $\operatorname{NE}\left(\mathcal{Y}_{s^{\prime}}\right)$, under the chosen identification. This invariance follows from the standard argument that (relative) Gromov-Witten invariants are deformation invariants, with a little care because our target spaces are open. For this, one considers the moduli space $\overline{\mathfrak{M}}\left(\tilde{\mathcal{Y}}^{o} / \mathcal{C}^{o}, \beta\right)$ of stable maps to $\tilde{\mathcal{Y}}^{o}$ relative to $\mathcal{C}^{o}$ and whose composition with $\pi$ is constant. Then one has a map $\psi: \overline{\mathfrak{M}}\left(\tilde{\mathcal{Y}}^{o} / \mathcal{C}^{o}, \beta\right) \rightarrow S$ whose fibre over $s$ is $\overline{\mathfrak{M}}\left(\tilde{\mathcal{Y}}_{s}^{o} / \mathcal{C}_{s}^{o}, \beta\right)$. Letting $\xi$ be the inclusion of this fibre in $\overline{\mathfrak{M}}\left(\tilde{\mathcal{Y}}^{o} / \mathcal{C}^{o}, \beta\right)$, deformation invariance will follow if we know that

$$
\xi^{!}\left[\overline{\mathfrak{M}}\left(\tilde{\mathcal{Y}}^{o} / \mathcal{C}^{o}, \beta\right)\right]^{v i r}=\left[\overline{\mathfrak{M}}\left(\tilde{\mathcal{Y}}_{s}^{o} / \mathcal{C}_{s}^{o}, \beta\right)\right]^{v i r}
$$

and $\psi$ is proper. The first statement is standard in Gromov-Witten theory, see e.g. the argument of Theorem 4.2 of [LT98] in the non-relative case, which carries over to the relative case. The second point, the properness of $\psi$, follows exactly as in the proof of Lemma 3.2 .

3.2. Consistency: Overview of the proof. We will describe in detail the intuition behind each step of the proof of consistency. In the next subsection, we will work somewhat more generally with a more general scattering diagram $\mathfrak{D}$ for certain steps, as this will be needed in $\mathrm{K} 3$. for the K3 case. However, for the discussion here let us assume we are only studying the consistency of the canonical scattering diagram.

Step I. We can replace $(Y, D)$ with a toric blow-up of $(Y, D)$. This is straightforward — toric blowups just correspond to refinements of $\Sigma$, but do not change broken lines or scattering diagrams. 
Step II. We can assume that $(Y, D)$ has a toric model and $P$ is a finitely generated submonoid of $A_{1}(Y, \mathbb{Z})$ containing $\mathrm{NE}(Y)$, with $\eta$ the inclusion. By Step I and Proposition 1.3 , we can assume $(Y, D)$ has a toric model. We can then always factor $\eta$ as $\mathrm{NE}(Y) \stackrel{\bar{\eta}}{\longrightarrow} \bar{P} \stackrel{\psi}{\longrightarrow} P$ where $\bar{P}$ is a finitely generated submonoid of $A_{1}(Y, \mathbb{Z})$ containing $\mathrm{NE}(Y)$ with $\bar{\eta}$ the inclusion. In this case there are two canonical scattering diagrams, $\overline{\mathfrak{D}}$ and $\mathfrak{D}$ defined using $\bar{\eta}: \mathrm{NE}(Y) \rightarrow \bar{P}$ and $\eta: \mathrm{NE}(Y) \rightarrow P$ respectively. Then $\mathfrak{D}$ can be obtained from $\overline{\mathfrak{D}}$ essentially just by applying $\psi$ to each exponent appearing in each function $f_{\mathfrak{d}}$.

In this case we show that if consistency holds for $\overline{\mathfrak{D}}$ then it holds for $\mathfrak{D}$. The idea is that given a broken line $\gamma$ for $\overline{\mathfrak{D}}$, we can get something like a broken line for $\mathfrak{D}$ by applying $\psi$ to the exponents of monomials attached to $\gamma$. However, this isn't necessarily a broken line for $\mathfrak{D}$. Indeed, there might be two different broken lines for $\overline{\mathfrak{D}}$, say $\gamma_{1}$ and $\gamma_{2}$, which after we apply $\psi$ give broken lines with the same sequence of attached exponents. These should not arise as distinct broken lines for $\mathfrak{D}$, and we have to combine the monomials attached to these broken lines. This requires a certain amount of book-keeping.

Step III. Reduction to the Gross-Siebert locus. By Step II we can assume we have a toric model $p: Y \rightarrow \bar{Y}$. Let $H$ be an ample divisor on $\bar{Y}$. Shrinking $P$ if necessary, we can assume that $P$ has a face of the form $P \cap\left(p^{*} H\right)^{\perp}$. Let $G$ be the monomial ideal which is the complement of this face, $E$ the subgroup of $P^{\text {gp }}$ generated by $P \backslash G$. The main work in this step is to show that we can replace $P$ by $P+E$. This requires a bit of analysis of the rays $\left(\rho_{i}, f_{\rho_{i}}\right)$ of $\mathfrak{D}^{\text {can }}$. In particular, we need to understand the contribution to $f_{\rho_{i}}$ coming from the exceptional curves of $p$ meeting $D_{i}$.

After doing this, we have $P^{\times}=E$, so now $X_{I, \mathfrak{D}}^{o}$ lives over the thickening of a torus $T^{\mathrm{gs}}$ we call the Gross-Siebert locus.

Step IV. Pushing the singularities to infinity. This is the crucial step, and we explain carefully the intuition here. In GS07], Gross and Siebert considered a smoothing construction associated to an integral affine manifold with singularities where (in the two-dimensional case) the singularities occurred only in the interior of edges of a polyhedral decomposition of $B$, rather than at the vertices. The case at hand, with one singularity at the origin, does not fit into that framework. In particular, in the GrossSiebert world, the singularities must have monodromy of the form $\left(\begin{array}{ll}1 & k \\ 0 & 1\end{array}\right)$ for some $k>0$, with the tangent line to the edge containing the singularity being the invariant direction; in analogy with the Kodaira classification, we call this an $I_{k}$ singularity. Indeed, one expects a cycle of $k$ two-spheres as fibre over such a point in the SYZ picture. 
Here, we can view such a surface as being obtained by factoring the complicated singularity $0 \in B$ into $I_{k}$ singularities along the edges of $\Sigma$. We should have an $I_{k_{i}}$ singularity on the ray $\rho_{i}$ where $k_{i}$ is the number of exceptional divisors of $p: Y \rightarrow \bar{Y}$ intersecting $D_{i}$. This process can be described as follows. Let $(\bar{B}, \bar{\Sigma})$ be the fan associated to $(\bar{Y}, \bar{D})$. There is a piecewise linear isomorphism

$$
\nu: B \rightarrow \bar{B}
$$

which identifies each cone in $\Sigma$ with the corresponding cone in $\bar{\Sigma}$. This is an isomorphism of integral affine manifolds outside of $\rho_{i}$, but it is not affine along $\rho_{i}$. There is a natural one-parameter family of integral affine manifolds interpolating between the two structures by a process Kontsevich and Soibelman [KS06] call moving worms. Precisely, choose points $y_{i} \in \rho_{i} \backslash\{0\}$. Let $\Delta:=\left\{y_{i} \mid 1 \leq i \leq n\right\}, B_{0}^{\prime}:=B \backslash \Delta$. Put a new affine structure on $B_{0}^{\prime}$ compatible with the affine structures on the interior of each maximal cell by defining a $\Sigma$-piecewise linear function to be linear if its restriction to a small neighbourhood of $\left(y_{i},+\infty\right) \subset \rho_{i}$ in $B_{0}^{\prime}$ is $B$-linear, and its restriction to a small neighbourhood of $\left[0, y_{i}\right) \subset \rho_{i}$ in $B_{0}^{\prime}$ is $\bar{B}$-linear. Call the resulting integral affine manifold with singularities $B^{\prime}$. The map $\nu: B^{\prime} \rightarrow \bar{B}$ is a linear isomorphism near 0 This new manifold can be seen to have an $I_{k_{i}}$ singularity at $y_{i}$, with invariant direction $\rho_{i}$.

Now if we were to apply the algorithm of Gross and Siebert GS07 to $B^{\prime}$, one would find roughly that one obtains a scattering diagram which initially has two rays emanating from each singularity. The rays emanating from $\rho_{i}$ are initially contained in $\rho_{i}$; one of these goes out to infinity and the other passes through the origin and then to infinity. Where all these rays meet at the origin, one must follow a procedure of Kontsevich and Soibelman [KS06] and add some additional rays to ensure that the composition of automorphisms associated to the rays about a loop centered at the origin is the identity. We then obtain a scattering diagram which can be shown is very close to the canonical scattering diagram, the only difference being the segments of the rays between the $y_{i}$ and the origin.

We do not actually work with this affine manifold with singularities. Rather, we instead push the singularities $y_{i}$ to infinity. In doing so, we replace $B^{\prime}$ with $\bar{B}$. We transfer the canonical scattering diagram $\mathfrak{D}$ to a scattering diagram $\overline{\mathfrak{D}}$ on $\bar{B}$, differing from $\mathfrak{D}$ essentially only by changing the rays supported on the $\rho_{i}$ 's in a simple way motivated by the above description. Once this is done, we show consistency of $\mathfrak{D}$ is equivalent to consistency of $\overline{\mathfrak{D}}$. Now we no longer have to deal with any singularities.

It is much easier to determine consistency when there are no singularities. In particular, we appeal to a result in [CPS], which shows that $\overline{\mathfrak{D}}$ is consistent provided that 
the composition of automorphisms associated to the rays about a loop centered at the origin is the identity. We say such a scattering diagram is compatible. The important point is that we can now make sense of such a statement: when we had a singularity at the origin, there was no common ring which the automorphisms associated to rays could act on. However, without a singularity at the origin, there are such rings, as appeared in [GS07.

Step $V . \overline{\mathfrak{D}}$ satisfies the required compatibility condition. This step is really the punchline, explaining why the particular choice of the canonical scattering diagram $\mathfrak{D}$ gives a diagram $\mathfrak{D}$ which is compatible. We make use of GPS09 to link the enumerative definition of $\mathfrak{D}$ to the notion of compatibility. Indeed, the definition of the canonical scattering diagram was originally obtained by working backwards from the enumerative description of [GPS09]. This connection is worked out in 93.4 .

3.3. Consistency: Reduction to the Gross-Siebert locus. We now begin the proof of Theorem 3.8, following the outline given in \$3.2. We will, however, prove a number of lemmas in a slightly more general context, as we will need some more general consistency results in [K3]. We assume we are given $(Y, D), \eta: \operatorname{NE}(Y) \rightarrow P$ and $\varphi$ defined as in Example 2.3, and a radical ideal $J \subseteq P$. Suppose we are given a scattering diagram $\mathfrak{D}$ for this data; the application in this paper will be $\mathfrak{D}=\mathfrak{D}^{\text {can }}$. In particular, the hypotheses of Theorem 3.8 imply $\mathfrak{D}^{\text {can }}$ is a scattering diagram for this data.

Step I. Replacing $(Y, D)$ with a toric blowup.

Proposition 3.10. Let $p:(\tilde{Y}, \tilde{D}) \rightarrow(Y, D)$ be a toric blowup. Then if we take $\tilde{\eta}:=\eta \circ p_{*}: \operatorname{NE}(\tilde{Y}) \rightarrow P$, then $\mathfrak{D}$ can also be viewed as a scattering diagram for $B_{(\tilde{Y}, \tilde{D})}, P$. Furthermore, if $\mathfrak{D}$ is consistent for this latter data, it is consistent for the data $B_{(Y, D)}, P$.

Proof. Decorate notation, writing for example $\tilde{B}, \tilde{\Sigma}$ for the singular affine manifold with subdivision into cones associated to $(\tilde{Y}, \tilde{D})$. By Lemma 1.6, we have a canonical identification of the underlying singular affine manifolds $B=\tilde{B}$, and $\tilde{\Sigma}$ is the refinement of $\Sigma$ obtained by adding one ray for each $p$-exceptional divisor. We have multivalued piecewise linear functions $\varphi$ on $B$ and $\tilde{\varphi}$ on $\tilde{B}$. We can in fact choose representatives so that $\tilde{\varphi}=\varphi$. Indeed, $\kappa_{\rho, \tilde{\varphi}}=\eta\left(p_{*}\left(\left[\tilde{D}_{\rho}\right]\right)\right)$ where $\tilde{D}_{\rho}$ is the irreducible component of $\tilde{D}$ corresponding to $\rho$. But $p_{*}\left(\left[\tilde{D}_{\rho}\right]\right)=0$ if $\rho \notin \Sigma$, and $p_{*}\left(\left[\tilde{D}_{\rho}\right]\right)=\left[D_{\rho}\right]$ if $\rho \in \Sigma$. Thus $\tilde{\varphi}$ in fact has the same domains of linearity as $\varphi$, and the same bending parameters, so we can choose representatives which agree.

As a consequence, we note that the sheaves $\mathcal{P}$ and $\tilde{\mathcal{P}}$ on $B_{0}$ defined using $\varphi$ and $\tilde{\varphi}$ coincide. Furthermore, if $\tilde{\tau} \subset \tilde{\sigma}$ are cones in $\tilde{\Sigma}$, with $\tau \in \Sigma$ the smallest cone containing 
$\tilde{\tau}$ and $\sigma \in \Sigma$ the smallest cone containing $\tilde{\sigma}$, there is a canonical identification of $P_{\varphi_{\tau}}$ with $P_{\tilde{\varphi}_{\tilde{\tau}}}$ and a canonical isomorphism

$$
\tilde{R}_{\tilde{\tau}, I} \cong R_{\tau, I}
$$

note the slightly non-trivial case when $\operatorname{dim} \tilde{\tau}=1$ but $\operatorname{dim} \tau=2$, in which case we use the fact that $\kappa_{\tilde{\tau}, \tilde{\varphi}}=0$.

Using these identifications, we can view $\mathfrak{D}$ as living on $\tilde{B}$, and as such, one sees from the definition that $\mathfrak{D}$ is a scattering diagram for the data $\tilde{B}, P, \tilde{\varphi}$.

Now suppose $\sqrt{I}=J$. One observes that the set of broken lines contributing to $\operatorname{Lift}_{Q}(q)$ are the same whether we are working in $B$ or $\tilde{B}$. Thus if $Q \in \tilde{\sigma} \in \tilde{\Sigma}_{\text {max }}$, $\operatorname{Lift}_{Q}(q) \in \tilde{R}_{\tilde{\sigma}, I}$, defined using $\tilde{B}$, coincides under the isomorphism (3.3) with $\operatorname{Lift}_{Q}(q) \in$ $R_{\sigma, I}$. From this one sees easily that if $\mathfrak{D}$ is consistent for $\tilde{Y}$, it is consistent for $Y$.

Corollary 3.11. Given $Y, P, \eta, J$ satisfying the hypotheses of Theorem 3.8, then Theorem 3.8 holds for this data if it holds for the data $\tilde{Y}, P, \tilde{\eta}, J$.

Proof. By the proposition, one just needs to check that the canonical scattering diagrams defined using $Y$ or $\tilde{Y}$ are identical. Indeed, given a ray $\mathfrak{d} \subset B$, we can choose a refinement $\Sigma^{\prime}$ of $\Sigma$ which is also a refinement of $\tilde{\Sigma}$, giving maps $\tilde{\pi}: Y^{\prime} \rightarrow \tilde{Y}$ and $\pi: Y^{\prime} \rightarrow Y$. Then for an $\mathbb{A}^{1}$-class $\beta \in A_{1}\left(Y^{\prime}, \mathbb{Z}\right), \eta\left(\pi_{*}(\beta)\right)=\tilde{\eta}\left(\tilde{\pi}_{*}(\beta)\right)$, and so $f_{\mathfrak{o}}$ is the same for $\tilde{Y}$ and $Y$.

Step II. Changing the monoid P.

We would like to change the monoid $P$, which was fairly arbitrary, to one with better properties. For this step, assume we are given monoid homomorphisms

$$
\mathrm{NE}(Y) \stackrel{\bar{\eta}}{\longrightarrow} \bar{P} \stackrel{\psi}{\longrightarrow} P
$$

with $\eta=\psi \circ \bar{\eta}$. Then $\eta$ and $\bar{\eta}$ induce multivalued piecewise linear functions $\varphi$ and $\bar{\varphi}$ respectively, via Example 2.3 , with $\varphi=\psi \circ \bar{\varphi}$. The monoids $P, \bar{P}$ and functions $\varphi, \bar{\varphi}$ yield sheaves $\mathcal{P}$ and $\overline{\mathcal{P}}$ over $B_{0}$. The map $\psi: \bar{P} \rightarrow P$ induces a map of sheaves $\psi: \overline{\mathcal{P}} \rightarrow \mathcal{P}$ using $\varphi=\psi \circ \bar{\varphi}$, and hence it also induces monoid homomorphisms $\psi: \bar{P}_{\bar{\varphi}_{\tau}} \rightarrow P_{\varphi_{\tau}}$ for any $\tau \in \Sigma \backslash\{0\}$.

Suppose $\overline{\mathfrak{D}}$ is a scattering diagram for the data $B, \bar{P}, \overline{\mathfrak{m}}=\bar{P} \backslash \bar{P}^{\times}$. For each ray

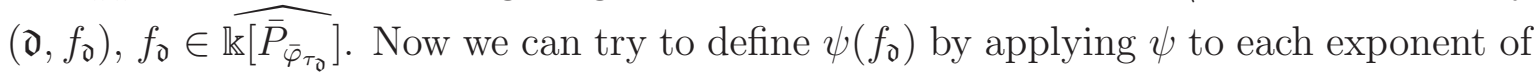
$f_{\mathfrak{o}}$, but in general, this need not make sense even formally since $\psi$ may take an infinite number of exponents occuring in $f_{\mathfrak{d}}$ to a single element of $P$. However, we shall write $\psi\left(f_{\mathfrak{d}}\right)$ for such an expression if it does make sense as an element of $\widehat{\mathbb{k}\left[P_{\varphi_{\tau_{\mathfrak{d}}}}\right]}$. If $\psi\left(f_{\mathfrak{d}}\right)$ makes sense for each $\left(\mathfrak{d}, f_{\mathfrak{d}}\right) \in \overline{\mathfrak{D}}$, we write

$$
\psi(\overline{\mathfrak{D}})=\left\{\left(\mathfrak{d}, \psi\left(f_{\mathfrak{d}}\right)\right) \mid\left(\mathfrak{d}, f_{\mathfrak{d}}\right) \in \overline{\mathfrak{D}}\right\} .
$$


Proposition 3.12. In the above situation, suppose $\overline{\mathfrak{D}}$ is a scattering diagram for the data $B, \bar{P}, \overline{\mathfrak{m}}=\bar{P} \backslash \bar{P}^{\times}$, such that $\mathfrak{D}=\psi(\overline{\mathfrak{D}})$ makes sense and is a scattering diagram for the data $B, P, J$, where $J$ is a radical ideal in $P$. Assume that $\kappa_{\rho, \varphi} \in J$ for at least one ray $\rho \in \Sigma$. If $\overline{\mathfrak{D}}$ is consistent for $\bar{P}, \bar{\eta}, \overline{\mathfrak{m}}$, then $\mathfrak{D}$ is consistent for $P, \eta, J$.

Proof. Let $q \in B_{0}(\mathbb{Z})$. Then if $\bar{\gamma}$ is a broken line for $q$ with endpoint $Q$ with respect to the barred data, i.e., $\bar{P}, \overline{\mathcal{P}}$ etc., we can construct what we shall call $\psi(\bar{\gamma})$. This will be the data required for defining a broken line for the unbarred data. The underlying map of $\psi(\bar{\gamma})$ coincides with that of $\bar{\gamma}$. For the attached monomials, we simply apply $\psi$ to the monomial $m_{L}(\bar{\gamma})$ attached to a domain of linearity $L$ of $\bar{\gamma}$ to get the attached monomial for $\psi(\bar{\gamma})$. This is not a broken line for the unbarred data, as condition (3) of Definition 2.16 need not hold. Indeed, when a broken line bends at a ray, the attached monomial will be replaced by a term in (2.9). However, there might be several different terms $c_{i} z^{\bar{s}_{i}}$ appearing in (2.9) with $\bar{s}_{i} \in \bar{P}_{\varphi_{\tau}}$ such that $\psi\left(\bar{s}_{i}\right)$ all coincide with some $s \in P_{\varphi_{\tau}}$. Each choice $c_{i} \bar{z}^{\bar{s}_{i}}$ leads to a different broken line $\bar{\gamma}_{i}$, but $\psi\left(\bar{\gamma}_{i}\right)$ is not a broken line because $c_{i} z^{\psi\left(\bar{s}_{i}\right)}=c_{i} z^{s}$ is not a term in the formula (2.9) for the monoid $P$. Rather, one needs to replace the collection of broken lines $\bar{\gamma}_{i}$ with a single one which has monomial $\sum_{i} c_{i} z^{s}$ attached after the bend. To deal with this, we need to do a certain amount of book-keeping.

Fix an ideal $I \subset P$ with $\sqrt{I}=J, Q \in \sigma \in \Sigma$, and let $\overline{\mathfrak{B}}$ be the set of broken lines $\bar{\gamma}$ for the barred data with endpoint $Q$ such that $\psi(\operatorname{Mono}(\bar{\gamma})) \notin I \cdot \mathbb{k}\left[P_{\varphi_{\sigma}}\right]$. The same finiteness argument of Lemma 2.25 shows that $\overline{\mathfrak{B}}$ is a finite set. Note this uses the facts (1) at least one $\kappa_{\rho, \varphi} \in J$ and (2) all but a finite number of monomials appearing in $\mathfrak{D}$ lie in $I$.

We define an equivalence relation on $\overline{\mathfrak{B}}$ by saying $\bar{\gamma}_{1} \sim \bar{\gamma}_{2}$ provided $\psi\left(\bar{\gamma}_{1}\right)$ and $\psi\left(\bar{\gamma}_{2}\right)$ coincide except possibly for the $\mathbb{k}$-valued coefficients of the monomials attached to the domains of linearity. Given an equivalence class $\xi \subset \overline{\mathfrak{B}}$ with respect to this equivalence relation, we will show there is at most one broken line $\gamma_{\xi}$ for the unbarred data such that

$$
\sum_{\bar{\gamma} \in \xi} \psi(\operatorname{Mono}(\bar{\gamma}))=\operatorname{Mono}\left(\gamma_{\xi}\right),
$$

with there being no such broken line precisely if the above quantity is zero. Furthermore, every broken line $\gamma$ for the unbarred data with $\operatorname{Mono}(\gamma) \notin I \cdot \mathbb{k}\left[P_{\varphi_{\sigma}}\right]$ arises in this way.

Define $\gamma_{\xi}$ to be the broken line with underlying piecewise linear map given by any element of $\xi$, with the following attached monomials. For any domain of linearity $L=[s, t]$ for $\gamma_{\xi}$, choose a maximal subset $\xi_{L} \subset \xi$ of broken lines such that the attached 
monomials for $\bar{\gamma}_{1}$ and $\bar{\gamma}_{2}$ on $(-\infty, t]$ do not coincide for any $\bar{\gamma}_{1}, \bar{\gamma}_{2} \in \xi_{L}$. Then define

$$
m_{L}\left(\gamma_{\xi}\right)=\sum_{\bar{\gamma} \in \xi_{L}} m_{L}(\psi(\bar{\gamma})) .
$$

Assuming that the final monomial attached to $\gamma_{\xi}$ is not zero, one checks easily that $\gamma_{\xi}$ is a broken line, now satisfying (3) of Definition 2.16, and (3.4) is satisfied since for $L$ the last domain of linearity of $\gamma_{\xi}$, one takes $\xi_{L}=\xi$. Furthermore, it is easy to see that any broken line for the unbarred data with the same underlying map and attached monomials at most differing by their coefficients from $\gamma_{\xi}$ must in fact coincide with $\gamma_{\xi}$. This shows the claim.

Since $\overline{\mathfrak{B}}$ is finite, there is some $k>0$ such that for any $\bar{\gamma} \in \overline{\mathfrak{B}}, \operatorname{Mono}(\bar{\gamma}) \in \mathbb{k}\left[\bar{P}_{\bar{\varphi}_{\tau}}\right]$ does not lie in $\overline{\mathfrak{m}}^{k} \cdot \mathbb{k}\left[\bar{P}_{\bar{\varphi}_{\tau}}\right]$. If we take $\bar{I}=\psi^{-1}(I)$, then it is clear from (3.4) that

$$
\psi\left(\overline{\operatorname{Lift}}_{Q}(q)\right)=\operatorname{Lift}_{Q}(q)
$$

where $\overline{\operatorname{Lift}}_{Q}(q)$ is the lift defined with respect to the ideal $\bar{I}$ and the other barred data, and $\operatorname{Lift}_{Q}(q)$ is defined with respect to the unbarred data and the ideal $I$. Now $\overline{\mathfrak{D}}$ is consistent for $\overline{\mathfrak{m}}$, which implies (1) and (2) of Definition 2.26 hold for the ideal $\overline{\mathfrak{m}}^{k}+\bar{I}$. Since any monomial in $\bar{P} \backslash \bar{I}$ appearing in $\overline{\operatorname{Lift}}_{Q}(q)$ is in $\bar{P} \backslash\left(\overline{\mathfrak{m}}^{k}+\bar{I}\right)$, we can use (3.5) to deduce consistency of $\mathfrak{D}$ from consistency of $\overline{\mathfrak{D}}$.

Step III. Reduction to the Gross-Siebert locus. As a consequence of Proposition 1.3 and Corollary 3.11, in order to prove Theorem 3.8 (i.e., with $\mathfrak{D}=\mathfrak{D}^{\text {can }}$ ), we may assume we have a toric model $p:(Y, D) \rightarrow(\bar{Y}, \bar{D})$ with $\bar{D}=\bar{D}_{1}+\cdots+\bar{D}_{n}$. Furthermore, by replacing $(Y, D)$ with a deformation equivalent pair and using Lemma 3.9, we can assume that $p$ is the blowup at distinct points $x_{i j}, 1 \leq j \leq \ell_{i}$, along $\bar{D}_{i}$, with exceptional divisors $E_{i j}$. Assume $D_{i}$ is the proper transform of $\bar{D}_{i}$, corresponding to the ray $\rho_{i} \in \Sigma$.

By Proposition 3.12, we can replace $P$ with a better suited choice of monoid. We shall do this as follows in the case that $\mathfrak{D}=\mathfrak{D}^{\text {can }}$. As in Example $\underline{3.6}$, the nef cone $\overline{\mathcal{K}}(Y) \subset$ $A^{1}(Y, \mathbb{R})$ contains a strictly convex rational polyhedral cone $\sigma$, so $\sigma^{\vee} \subset A_{1}(Y, \mathbb{R})$ is a strictly convex rational polyhedral cone containing $\mathrm{NE}(Y)$. The map $\eta: \mathrm{NE}(Y) \rightarrow P$ induces a map $\eta: A_{1}(Y, \mathbb{R}) \rightarrow P_{\mathbb{R}}^{\mathrm{gp}}$. Since $P$ is toric, there is some rational polyhedral cone $\sigma_{P} \subset P_{\mathbb{R}}^{\mathrm{gp}}$ such that $P=\sigma_{P} \cap P^{\mathrm{gp}}$. In addition, let $H$ be an ample divisor on $\bar{Y}$, so that $\mathrm{NE}(Y) \cap\left(p^{*} H\right)^{\perp}$ is a face of $\mathrm{NE}(Y)$, generated by the classes $\left[E_{i j}\right]$. Now take

$$
\sigma_{\bar{P}}=\eta^{-1}\left(\sigma_{P}\right) \cap \sigma^{\vee} \cap\left\{q \in A_{1}(Y, \mathbb{R}) \mid p^{*} H \cdot q \geq 0\right\},
$$

and take

$$
\bar{P}=\sigma_{\bar{P}} \cap A_{1}(Y, \mathbb{Z})
$$


As $\sigma_{\bar{P}}$ is strictly convex, $(\bar{P})^{\times}=\{0\}, \overline{\mathfrak{m}}=\bar{P} \backslash\{0\}$, and if $\bar{I}$ is an $\overline{\mathfrak{m}}$-primary ideal, $\bar{P} \backslash \bar{I}$ is finite. Thus the hypotheses of Theorem 3.8 trivially hold for $\bar{\eta}: \mathrm{NE}(Y) \rightarrow \bar{P}$. By Proposition 3.12, we can replace $P$ with $\bar{P}$ to prove Theorem 3.8 .

The above discussion shows that in order to complete a proof of consistency of $\mathfrak{D}^{\text {can }}$, (i.e., Theorem 3.8 ), we can operate under the following assumptions:

Assumptions 3.13. $\quad$ - There is a toric model

$$
p:(Y, D) \rightarrow(\bar{Y}, \bar{D})
$$

which blows up distinct points $x_{i j}$ on $D_{i}$, with exceptional divisors $E_{i j}$.

- $\eta: \mathrm{NE}(Y) \rightarrow P$ is an inclusion, and $P^{\times}=\{0\}$. Via Example 2.3, this gives the function $\varphi$.

- There is a face of $P$ whose intersection with $\mathrm{NE}(Y)$ is $\mathrm{NE}(Y) \cap\left(p^{*} H\right)^{\perp}$. Let $G$ be the prime monomial ideal given by the complement of this face. Note that $G \neq \mathfrak{m}$ unless $p$ is an isomorphism.

- $J=\mathfrak{m}=P \backslash\{0\}$.

- $\mathfrak{D}$ is a scattering diagram for the data $P, \varphi$ and $J$.

Definition 3.14. The Gross-Siebert locus is the open torus orbit $T^{\mathrm{gs}}$ of $\mathrm{Spec} \mathbb{k}[P] / G$.

We now want to work not with the maximal ideal $\mathfrak{m}$ but with the ideal $G$, effectively extending the families $X_{I, \mathfrak{D}}^{o}$ with $\sqrt{I}=\mathfrak{m}$ to infinitesimal neighbourhoods of the toric boundary stratum of $\operatorname{Spec} \mathbb{k}[P]$ associated to $G$. We will then find it easier to check the explicit equalities of Definition 2.26 after restricting to these neighbourhoods of the Gross-Siebert locus. To do so requires showing that the diagram $\mathfrak{D}$ we are working with ( $\mathfrak{D}^{\text {can }}$ in this paper) is also a scattering diagram for the data $P, \eta, G$. In the case of $\mathfrak{D}^{\text {can }}$, this requires analyzing elements of this scattering diagram supported on the $\rho_{i}$.

We first perform this analysis for $\mathfrak{D}^{\text {can }}$; we will then continue our proof assuming that the elements of $\mathfrak{D}$ supported on the rays $\rho_{i}$ take the same form as the corresponding elements of $\mathfrak{D}^{\text {can }}$ modulo $G$.

For each ray $\rho_{i}$ in $\Sigma$, we have a unique ray $\left(\rho_{i}, f_{\rho_{i}}\right) \in \mathfrak{D}^{\text {can }}$ with support $\rho_{i}$. The following describes $f_{\rho_{i}} \bmod G$.

Lemma 3.15. Given Assumption 3.13 with $\mathfrak{D}=\mathfrak{D}^{\text {can }}$, viewing $f_{\rho_{i}}$ as an element of $\mathbb{k}\left[P_{\varphi_{\rho_{i}}}\right] \otimes R_{I}$ with $\sqrt{I}=\mathfrak{m}$, we have

$$
f_{\rho_{i}}=g_{\rho_{i}} \prod_{j=1}^{\ell_{i}}\left(1+b_{i j} X_{i}^{-1}\right)
$$


where $b_{i j}=z^{\eta\left(\left[E_{i j}\right]\right)}$ and $g_{\rho_{i}} \equiv 1 \bmod G$. The $j^{\text {th }}$ term of the product is the contribution from $\mathbb{A}^{1}$-classes coming from multiple covers of the $p$-exceptional divisor $E_{i j}$, and $g_{\rho_{i}}$ is the product of contributions from all other $\mathbb{A}^{1}$-classes.

Proof. Note that in defining $f_{\rho_{i}}$ using the definition of the canonical scattering diagram, we take $\tilde{Y}=Y$. Now the only terms that contribute to $f_{\rho_{i}} \bmod G$ will involve classes $\beta \in \mathrm{NE}(Y) \subset A_{1}(Y)$ with $\eta(\beta) \notin G$, so in particular, such a $\beta$ must be a linear combination $\sum_{j=1}^{\ell_{i}} c_{j}\left[E_{i j}\right]$, with $k_{\beta}=\sum c_{j}$. Furthermore, if $f: C \rightarrow Y$ contributes to $N_{\beta}, f(C)$ must be contained in $\bigcup_{i, j} E_{i j}$. Indeed, if $f(C)$ has an irreducible component $C^{\prime}$ not contained in this set, then $\eta\left(\left[C^{\prime}\right]\right) \in G$, so $\eta\left(f_{*}([C])\right) \in G$, as $G$ is an ideal. But $\eta\left(f_{*}([C])\right)=\eta(\beta)$, which we have assumed is not an element of $G$.

Since $f(C)$ is connected and intersects $D_{i}$, we now see that the image of $f$ is $E_{i j}$ for some $j$, and in particular, $f$ is a degree $k_{\beta}$ cover of $E_{i j}$. Then Theorem 6.1 of [GPS09] tells us that the contribution from $k_{\beta}$-fold multiple covers of $E_{i j}$ is $(-1)^{k_{\beta}-1} / k_{\beta}^{2}$. From this we conclude that

$$
\begin{aligned}
f_{\rho_{i}} & =\exp \left(h+\sum_{k=1}^{\infty} \sum_{j=1}^{\ell_{i}} k\left(\frac{(-1)^{k-1}}{k^{2}}\right)\left(b_{i j} X_{i}^{-1}\right)^{k}\right) \\
& =\exp (h) \prod_{j=1}^{\ell_{i}}\left(1+b_{i j} X_{i}^{-1}\right)
\end{aligned}
$$

where $h \equiv 0 \bmod G$. We take $g_{\rho_{i}}=\exp (h)$.

Corollary 3.16. $\mathfrak{D}^{\text {can }}$ is a scattering diagram for the data $(B, \Sigma), P, \varphi$ and $G$.

Proof. Fixing an $I \subset P$ with $\sqrt{I}=G$, there exists a bound $n$ such that $q \in P \backslash I$ implies $q \cdot p^{*} H<n$, where $H$ is a fixed ample divisor on $\bar{Y}$. Thus if $\beta$ is an $\mathbb{A}^{1}$ class with $\eta\left(\pi_{*}(\beta)\right) \in P \backslash I$, there are only a finite number of choices for $p_{*} \pi_{*} \beta$. We need to examine the possible choices for $\pi_{*} \beta$. Given a choice for $\alpha=p_{*} \pi_{*} \beta$, we have $\pi_{*} \beta=p^{*} \alpha+\sum a_{i j} E_{i j}$ for some collection of $a_{i j} \in \mathbb{Z}$. Clearly the $a_{i j}$ are bounded below by the requirement that $\left(\pi_{*} \beta\right) \cdot D_{i} \geq 0$ for each $i$. On the other hand, if $a_{i j}>0$ for some $i, j$, then $\left(\pi_{*} \beta\right) \cdot E_{i j}<0$, so if $f: C \rightarrow Y$ is an $\mathbb{A}^{1}$-curve with $f_{*}[C]=\pi_{*} \beta$ then its reduced image $C^{\prime}$ must contain $E_{i j}$. (Technically a relative stable map in $\tilde{Y}^{\circ}$ is a map to an expanded degeneration of $\tilde{Y}^{\circ}$, but we compose with the projection to $\tilde{Y}^{\circ}$ and then the natural map $\tilde{Y}^{\circ} \rightarrow Y$.) Write $C^{\prime}=C^{\prime \prime} \cup E_{i j}$, with $C^{\prime \prime}$ a reduced divisor distinct from $E_{i j}$. Suppose $C^{\prime \prime}$ is non-empty. Necessarily either $C^{\prime \prime} \cap D$ is empty or $C^{\prime \prime}$ intersects $D$ only at $E_{i j} \cap D$; otherwise $C^{\prime}$ cannot be the image of a relative stable map with one point of tangency with $D$. In either case there is an integer $k$ such that $\left.\mathcal{O}_{Y}\left(C^{\prime \prime}+k E_{i j}\right)\right|_{D}$ is the trivial sheaf. However, by GHK12, Proposition 4.1, for a general deformation $\left(Y^{\prime}, D\right)$ of $(Y, D)$, the kernel of the restriction map Pic $Y^{\prime} \rightarrow \operatorname{Pic} D$ 
is trivial. Thus by Lemma 3.9, $N_{\beta}=0$. We conclude that there are only a finite number of choices of $\pi_{*} \beta$, except when $\pi_{*} \beta$ is a multiple of some $E_{i j}$. This shows condition (4) in Definition 2.13 of scattering diagrams, as well as condition (2). Note that $\kappa_{\rho_{i}, \varphi}=\left[D_{i}\right] \in G$ for each $i$ so condition (3) is vacuous for $\operatorname{dim} \tau_{\mathfrak{0}}=1$. If $\operatorname{dim} \tau_{\mathfrak{o}}=2$, any contributing $\mathbb{A}^{1}$-class $\beta$ satisfies $\pi_{*} \beta \in G$, so (3) holds.

Theorem 3.17. We follow the above notation. If $\mathfrak{D}^{\text {can }}$ is consistent as a scattering diagram for $(B, \Sigma), P, \varphi$, and $G$, then Theorem 3.8 is true.

Proof. This just follows from the series of reductions of Theorem 3.8 already made and the observation that if $I^{\prime}$ is an $\mathfrak{m}$-primary ideal, then since $G \subset \mathfrak{m}$ one can find some $k$ such that $k G \subset I^{\prime}$. To show consistency holds for the ideal $I^{\prime}$, we use the assumed consistency to observe consistency holds for the ideal $I=k G$, and this gives the desired result.

Remark 3.18. Given a consistent scattering diagram $\mathfrak{D}$ for $(B, \Sigma), P, \varphi$, and $G$, and $\kappa_{\rho, \varphi} \in G$ for all rays $\rho \in \Sigma$, Theorem 2.28 shows that with $\sqrt{I}=G$,

$$
X_{I}:=\operatorname{Spec} \Gamma\left(X_{I, \mathfrak{D}}^{o}, \mathcal{O}_{X_{I, \mathcal{D}}^{o}}\right)
$$

is flat over Spec $\mathbb{k}[P] / I$, and $X_{G}=\mathbb{V}_{n} \times \operatorname{Spec} \mathbb{k}[P] / G$.

Let $T^{\mathrm{gs}} \subset \operatorname{Spec} \mathbb{k}[P] / G$ be the Gross-Siebert locus, Definition 3.14. Note $T^{\mathrm{gs}}$ determines open subschemes of the thickenings $\operatorname{Spec} \mathbb{k}[P] / I$, which we will shall denote by $T_{I}^{\mathrm{gs}}$.

We can describe the subscheme $T_{I}^{\mathrm{gs}}$ of $\operatorname{Spec} \mathbb{k}[P] / I$ as follows. Let $E \subset P^{\mathrm{gp}}$ be the lattice generated by the face $P \backslash G$. Then as a subset of $\operatorname{Spec} \mathbb{k}[P] / G, T^{\mathrm{gs}} \cong \operatorname{Spec} \mathbb{k}[E]$. Furthermore, if we take the localization $P+E$ of $P$ along the face $P \backslash G$, then $T_{I}^{\mathrm{gs}}$ as a subscheme of $\operatorname{Spec} \mathbb{k}[P] / I$ is $\operatorname{Spec} \mathbb{k}[P+E] /(I+E)$.

Note that $\mathfrak{m}_{P+E}=(P+E) \backslash E$, and $G=P \cap \mathfrak{m}_{P+E}$, so we can write $\mathbb{k}[E]=$ $\mathbb{k}[P+E] / \mathfrak{m}_{P+E}$.

We can now view $\varphi$ as a multivalued strictly $(P+E)$-convex function. Then we have the following obvious

Lemma 3.19. Suppose $\mathfrak{D}$ is a consistent scattering diagram for the data $(B, \Sigma), P+E$, $\varphi, \mathfrak{m}_{P+E}$, and a scattering diagram for the data $(B, \Sigma), P, \varphi$, and $G$. Then $\mathfrak{D}$ is also consistent as a scattering diagram for the latter data. In particular, by Theorem 3.17 , Theorem 3.8 holds if $\mathfrak{D}^{\text {can }}$ is consistent as a scattering diagram for $P+E, \mathfrak{m}_{P+E}$.

Proof. Since $P \cap \mathfrak{m}_{P+E}=G$, the equalities in Definition 2.26 can be tested for an ideal $I$ of $P$ with $\sqrt{I}=G$ by choosing some ideal $I^{\prime} \subseteq P+E$ with $\sqrt{I^{\prime}}=\mathfrak{m}_{P+E}$ and $I^{\prime} \cap P \subset I$. Then the equalities of Definition 2.26 hold for the data $P+E$ and $I^{\prime}$ by the assumed consistency, and hence also for $P$ and $I$. 
Let $\bar{I} \subset \mathfrak{m}_{P+E}$ be an ideal with $\sqrt{\bar{I}}=\mathfrak{m}_{P+E}$. Set $I=\bar{I} \cap P$. Then $X_{I, \mathfrak{D}}^{o}$ is flat over $\operatorname{Spec} \mathbb{k}[P] / I$. Restricting $X_{I, \mathfrak{D}}^{o}$ to the open set $\operatorname{Spec} \mathbb{k}[P+E] / \bar{I}$ gives the flat family $X_{\bar{I}, \mathfrak{D}}^{o}$.

We now replace $P$ by $P+E$ and $J$ by $\mathfrak{m}_{P+E}$ in what follows. We now summarize our current situation with the following assumptions:

Assumptions 3.20. $\quad$ - There is a toric model

$$
p:(Y, D) \rightarrow(\bar{Y}, \bar{D})
$$

which blows up distinct points $x_{i j}$ on $D_{i}, 1 \leq j \leq \ell_{i}$, with exceptional divisors $E_{i j}$.

- $\eta: \mathrm{NE}(Y) \rightarrow P$ is an inclusion. Via Example 2.3, this gives the function $\varphi$. $E=P^{\times}=P \cap\left(p^{*} H\right)^{\perp}$ is generated by the classes of exceptional curves of $p$. Let $G=P \backslash E=\mathfrak{m}_{P}$.

- $\mathfrak{D}$ is a scattering diagram for the data $P, \varphi$ and $G$. Furthermore, for each ray $\rho_{i} \in \Sigma$, the unique outgoing ray $\left(\rho_{i}, f_{\rho_{i}}\right) \in \mathfrak{D}$ satisfies

$$
f_{\rho_{i}}=g_{\rho_{i}} \prod_{j=1}^{\ell_{i}}\left(1+b_{i j} X_{i}^{-1}\right)
$$

with $g_{\rho_{i}} \equiv 1 \bmod G$ and $b_{i j}=z^{\left[E_{i j}\right]}$.

We note we have shown that $\mathfrak{D}=\mathfrak{D}^{\text {can }}$ achieves these assumptions.

Step IV. Pushing the singularities to infinity. We work with Assumptions 3.20. Consider the tropicalisation $(\bar{B}, \bar{\Sigma})$ of $(\bar{Y}, \bar{D})$. By Example 1.7, $\bar{B}$ in fact has no singularity at the origin, and is affine isomorphic to $M_{\mathbb{R}}=\mathbb{R}^{2}$ (with $M=\mathbb{Z}^{2}$ ), while $\bar{\Sigma}$ is precisely the fan for $\bar{Y}$. In order to distinguish between constructions on $(Y, D)$ and $(\bar{Y}, \bar{D})$, we decorate all existing notation with bars. For example, if $\tau \in \Sigma$, denote the corresponding cone of $\bar{\Sigma}$ by $\bar{\tau}$. Let $\bar{\varphi}$ be the multivalued $P_{\mathbb{R}}^{\mathrm{gp}}$-valued function on $\bar{B}$ such that

$$
\kappa_{\bar{\rho}, \bar{\varphi}}=p^{*}\left[\bar{D}_{\bar{\rho}}\right]
$$

Note that by Lemma 1.13, we can assume $\bar{\varphi}$ is in fact a single-valued function on $M_{\mathbb{R}}$. This single-valuedness will be important to be able to apply the method of Kontsevich and Soibelman, Theorem 3.23 .

We now have sheaves $\overline{\mathcal{P}}$ on $\bar{B}_{0}$ and $\mathcal{P}$ on $B_{0}$, induced by the two functions $\bar{\varphi}$ and $\varphi$ respectively.

Note that since $\bar{\varphi}$ is single-valued and $\bar{B}$ has no singularities, $\overline{\mathcal{P}}$ is the constant sheaf with fibre $P^{g p} \oplus M$. 
There is a canonical piecewise linear map

$$
\nu: B \rightarrow \bar{B}
$$

which restricts to an integral affine isomorphism $\left.\nu\right|_{\sigma}: \sigma \rightarrow \bar{\sigma}$, where $\sigma \in \Sigma_{\max }$ and $\bar{\sigma} \in \bar{\Sigma}_{\max }$ is the corresponding cell of $\bar{\Sigma}$. Note this map identifies $B(\mathbb{Z})$ with $\bar{B}(\mathbb{Z})$.

For each maximal cone $\sigma \in \Sigma_{\max }$, the derivative $\nu_{*}$ of $\nu$ induces a canonical identification of $\Lambda_{B, \sigma}$ with $\Lambda_{\bar{B}, \bar{\sigma}}$. This then gives an induced isomorphism of monoids:

$$
\tilde{\nu}_{\sigma}: P_{\varphi_{\sigma}} \rightarrow P_{\overline{\varphi_{\bar{\sigma}}}}
$$

given by

$$
\varphi_{\sigma}(m)+p \mapsto \bar{\varphi}_{\bar{\sigma}}\left(\nu_{*}(m)\right)+p,
$$

for $p \in P$ and $m \in \Lambda_{\sigma}$. This identifies the $\mathbb{k}[P]$-algebras $\mathbb{k}\left[P_{\varphi_{\sigma}}\right]$ and $\mathbb{k}\left[P_{\bar{\varphi} \overline{\bar{\sigma}}}\right]$, and the completions $\widehat{\mathbb{k}\left[P_{\varphi_{\sigma}}\right]}$ and $\widehat{\mathbb{k}\left[P_{\bar{\varphi}_{\bar{\sigma}}}\right]}$.

Because the map $\nu$ is only piecewise linear around rays $\rho \in \Sigma$, there is only a piecewise linear identification of $P_{\varphi_{\rho}}$ with $P_{\overline{\bar{\varphi}_{\bar{p}}}}$ and hence no identification of the corresponding rings. However, $\nu_{*}$ is still defined on the tangent space to $\rho$, and there is an identification

$\tilde{\nu}_{\rho}:\left\{\varphi_{\rho}(m)+p \mid m\right.$ is tangent to $\left.\rho, p \in P\right\} \rightarrow\left\{\bar{\varphi}_{\bar{\rho}}(m)+p \mid m\right.$ is tangent to $\left.\bar{\rho}, p \in P\right\}$ given by

$$
\varphi_{\rho}(m)+p \mapsto \bar{\varphi}_{\bar{\rho}}\left(\nu_{*}(m)\right)+p .
$$

We now explain the Kontsevich-Soibelman lemma. This has to do with scattering diagrams on the smooth affine surface $M_{\mathbb{R}}=\mathbb{R}^{2}$ (such as $\bar{B}=B_{(\bar{Y}, \bar{D})}$ ). For this general discussion, we fix the data of a monoid $Q$ which comes along with a map $r: Q \rightarrow M$. Let $\mathfrak{m}_{Q}=Q \backslash Q^{\times}$, and let $\widehat{\mathbb{k}[Q]}$ denote the completion of $\mathbb{k}[Q]$ with respect to the monomial ideal $\mathfrak{m}_{Q}$. (In our application we take $Q=P_{\bar{\varphi}}$ as defined in (1.5).)

We can then consider a variant of the notion of scattering diagram:

Definition 3.21. We define a scattering diagram for the pair $Q, r: Q \rightarrow M$. This is a set

$$
\mathfrak{D}=\left\{\left(\mathfrak{d}, f_{\mathfrak{d}}\right)\right\}
$$

where

- $\mathfrak{d} \subset M_{\mathbb{R}}$ is given by

$$
\mathfrak{d}=-\mathbb{R}_{\geq 0} m_{0}
$$

if $\mathfrak{d}$ is an outgoing ray and

$$
\mathfrak{d}=\mathbb{R}_{\geq 0} m_{0}
$$

if $\mathfrak{d}$ is an incoming ray, for some $m_{0} \in M \backslash\{0\}$. 
- $f_{\mathfrak{d}} \in \widehat{\mathbb{K}[Q]}$.

- $f_{\mathfrak{d}} \equiv 1 \bmod \mathfrak{m}_{Q}$.

- $f_{\mathfrak{d}}=1+\sum_{p} c_{p} z^{p}$ for $c_{p} \in \mathbb{k}, r(p) \neq 0$ a positive multiple of $m_{0}$.

- For any $k>0$, there are only a finite number of rays $\left(\mathfrak{d}, f_{\mathfrak{d}}\right) \in \mathfrak{D}$ with $f_{\mathfrak{o}} \not \equiv 1$ $\bmod \mathfrak{m}_{Q}^{k}$.

Definition 3.22. Given a loop $\gamma$ in $M_{\mathbb{R}}$ around the origin, we define the path ordered product

$$
\theta_{\gamma, \mathfrak{D}}: \widehat{\mathbb{k}[Q]} \rightarrow \widehat{\mathbb{k}[Q]}
$$

as follows. For each $k>0$, let $\mathfrak{D}[k] \subset \mathfrak{D}$ be the subset of rays $\left(\mathfrak{d}, f_{\mathfrak{d}}\right) \in \mathfrak{D}$ with $f_{\mathfrak{d}} \not \equiv 1$ $\bmod \mathfrak{m}_{Q}^{k}$. This set is finite. For $\mathfrak{d} \in \mathfrak{D}[k]$ with $\gamma\left(t_{0}\right) \in \mathfrak{d}$, define

$$
\theta_{\gamma, \mathfrak{d}}^{k}: \mathbb{k}[Q] / \mathfrak{m}_{Q}^{k} \rightarrow \mathbb{k}[Q] / \mathfrak{m}_{Q}^{k}
$$

by

$$
\theta_{\gamma, \mathfrak{d}}^{k}\left(z^{q}\right)=z^{q} f_{\mathfrak{d}}^{\left\langle n_{\mathfrak{d}}, r(q)\right\rangle}
$$

for $n_{\mathfrak{d}} \in M^{*}$ primitive satisfying, with $m$ a non-zero tangent vector of $\mathfrak{d}$,

$$
\left\langle n_{\mathfrak{d}}, m\right\rangle=0, \quad\left\langle n_{\mathfrak{d}}, \gamma^{\prime}\left(t_{0}\right)\right\rangle<0 .
$$

Then, if $\gamma$ crosses the rays $\mathfrak{d}_{1}, \ldots, \mathfrak{d}_{n}$ in order with $\mathfrak{D}[k]=\left\{\mathfrak{d}_{1}, \ldots, \mathfrak{d}_{n}\right\}$, we can define

$$
\theta_{\gamma, \mathfrak{D}}^{k}=\theta_{\gamma, \mathfrak{d}_{n}}^{k} \circ \cdots \circ \theta_{\gamma, \mathfrak{d}_{1}}^{k} .
$$

We then define $\theta_{\gamma, \mathfrak{D}}$ by taking the limit as $k \rightarrow \infty$.

The following is a slight generalisation of a result of Kontsevich and Soibelman which appeared in [KS06].

Theorem 3.23. Let $\mathfrak{D}$ be a scattering diagram in the sense of Definition 3.21. Then there is another scattering diagram Scatter $(\mathfrak{D})$ containing $\mathfrak{D}$ such that $\operatorname{Scatter}(\mathfrak{D}) \backslash \mathfrak{D}$ consists only of outgoing rays and $\theta_{\gamma, \operatorname{Scatter}(\mathfrak{D})}$ is the identity for $\gamma$ a loop around the origin.

For a proof of this theorem essentially as stated here, see GPS09, Theorem 1.4. The result is unique if $\operatorname{Scatter}(\mathfrak{D}) \backslash \mathfrak{D}$ has at most one ray in each possible direction; we shall assume Scatter( $\mathfrak{D})$ has been chosen to have this property. This can always be done.

We apply this in the following situation. We take $Q$ to be the monoid $P_{\bar{\varphi}}$ which yields the Mumford degeneration associated to the data $(\bar{B}, \bar{\Sigma}), \bar{\varphi}$ (recalling $\bar{B}=M_{\mathbb{R}}$ ), defined by

$$
P_{\bar{\varphi}}=\{(m, \bar{\varphi}(m)+p) \mid m \in M, p \in P\} \subset M \times P^{g p} .
$$

This comes with a canonical map $r: P_{\bar{\varphi}} \rightarrow M$ by projection. 
Definition 3.24. Suppose we are in the situation of Assumptions 3.20, We define a scattering diagram $\nu(\mathfrak{D})$ on $\bar{B}$ as follows. For every ray $\left(\mathfrak{d}, f_{\mathfrak{d}}\right) \in \mathfrak{D}$ not equal to $\left(\rho_{i}, f_{\rho_{i}}\right)$ for some $i, \nu(\mathfrak{D})$ contains the ray $\left(\nu(\mathfrak{d}), \tilde{\nu}_{\tau_{\mathfrak{d}}}\left(f_{\mathfrak{d}}\right)\right)$, and for each ray $\left(\rho_{i}, f_{\rho_{i}}\right)$, $\nu(\mathfrak{D})$ contains two rays, $\left(\bar{\rho}_{i}, \tilde{\nu}_{\tau_{\mathfrak{D}}}\left(g_{\rho_{i}}\right)\right)$ and $\left(\bar{\rho}_{i}, \prod_{j=1}^{\ell_{i}}\left(1+b_{i j}^{-1} \bar{X}_{i}\right)\right)$.

We note that $\nu(\mathfrak{D})$ may not actually be a scattering diagram in the sense of Definition 3.21, as it is possible that $f_{\mathfrak{o}} \notin \widehat{\mathbb{k}\left[P_{\bar{\varphi}}\right]}$ : if $p \in P_{\varphi_{\tau}}$, then $\tilde{\nu}_{\tau}(p) \in P_{\bar{\varphi}_{\tau}}$ but need not lie in $P_{\bar{\varphi}}$.

In the case of $\mathfrak{D}=\mathfrak{D}^{\text {can }}$, we can use the Kontsevich-Soibelman lemma to describe $\nu\left(\mathfrak{D}^{\text {can }}\right)$. This will both show that $\nu\left(\mathfrak{D}^{\text {can }}\right)$ is a scattering diagram in the sense of Definition 3.21 and that it satisfies an important additional property, namely the condition that $\theta_{\gamma, \nu\left(\mathfrak{D}^{\text {can }}\right)}$ is equal to the identity. This will allow us to prove consistency. Let

$$
\overline{\mathfrak{D}}_{0}=\left\{\left(\bar{\rho}_{i}, \prod_{j=1}^{\ell_{i}}\left(1+b_{i j}^{-1} \bar{X}_{i}\right)\right) \mid 1 \leq i \leq n\right\} .
$$

Let $\mathfrak{m}_{\bar{\varphi}}=P_{\bar{\varphi}} \backslash P_{\bar{\varphi}}^{\times}$as usual. Then by the strict convexity of $\bar{\varphi}, \bar{X}_{i} \in \mathfrak{m}_{\bar{\varphi}}$ so that $\overline{\mathfrak{D}}_{0}$ is a scattering diagram for the pair $P_{\bar{\varphi}}, r$ in the sense of Definition 3.21. Now define

$$
\overline{\mathfrak{D}}:=\operatorname{Scatter}\left(\overline{\mathfrak{D}}_{0}\right)
$$

where we require $\overline{\mathfrak{D}} \backslash \overline{\mathfrak{D}}_{0}$ to have only one outgoing ray in each direction (and no incoming rays).

The following will be Step V, which we defer until $\$ 3.4$.

Theorem 3.25. $\overline{\mathfrak{D}}=\nu\left(\mathfrak{D}^{\text {can }}\right)$. In particular, $\nu\left(\mathfrak{D}^{\text {can }}\right)$ is a scattering diagram in the sense of Definition 3.21 and $\theta_{\gamma, \overline{\mathfrak{D}}} \equiv 1$ for a loop $\gamma$ around the origin.

Example 3.26. Continuing with Example 3.7, note that the pair $(Y, D)$ can be obtained from the toric pair $(\bar{Y}, \bar{D})$ defined by the fan $\bar{\Sigma}$ with rays generated by $(1,0)$, $(1,1),(0,1),(-1,0)$ and $(0,-1)$, corresponding to $\bar{D}_{1}, \ldots, \bar{D}_{5}$, by blowing up one point on each of $\bar{D}_{4}$ and $\bar{D}_{5}$. This description determines $\overline{\mathfrak{D}}_{0}$ and hence $\overline{\mathfrak{D}}$. One can check this description agrees with that given in Example 3.7 for $\mathfrak{D}^{\text {can }}$, see e.g. GPS09, Example 1.6 for a similar computation.

Returning to the situation of Assumptions [3.20, suppose in addition that $\nu(\mathfrak{D})$ is a scattering diagram in the sense of Definition 3.21. (For example, by Theorem 3.25, $\mathfrak{D}=\mathfrak{D}^{\text {can }}$ satisfies these assumptions.) For $I \subset P$ an ideal with $\sqrt{I}=J$, we now have deformations $X_{I, \mathfrak{D}}^{o}$ and $\bar{X}_{I, \nu(\mathfrak{D})}^{o}$. The latter scheme is glued from open sets

$$
\bar{U}_{\bar{\rho}, I}=\operatorname{Spec} \bar{R}_{\bar{\rho}, I}
$$


along open sets identified with Spec $\bar{R}_{\bar{\sigma}, I}$. Here we are decorating the rings coming from the data on $\bar{B}$ with bars as before, while we maintain the notation $R_{\rho, I}$, etc., for those rings coming from the data on $B$.

Lemma 3.27. Given Assumptions [3.20, assume also that $\nu(\mathfrak{D})$ is a scattering diagram in the sense of Definition 3.21. Then there are isomorphisms

$$
p_{i}: R_{\rho_{i}, I} \rightarrow \bar{R}_{\bar{\rho}_{i}, I}
$$

and

$$
p_{i-1, i}: R_{\sigma_{i-1, i} I} \rightarrow \bar{R}_{\bar{\sigma}_{i-1, i}, I}
$$

for all $i$ such that the diagrams

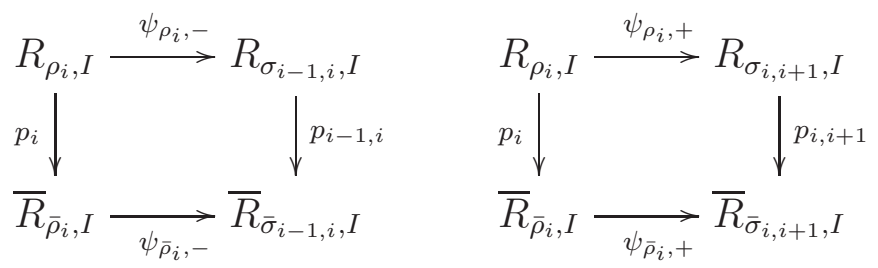

and

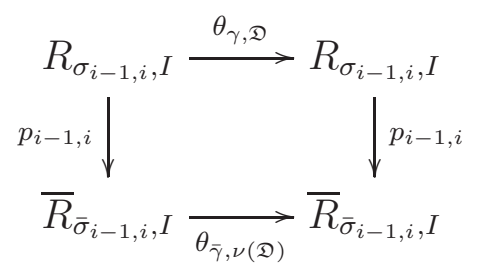

are commutative, where $\gamma$ is any path in $\sigma_{i-1, i}$ for which $\theta_{\gamma, \mathfrak{D}}$ is defined, and $\bar{\gamma}=\nu \circ \gamma$.

Consequently, the maps $p_{i}$ and $p_{i-1, i}$ induce an isomorphism

$$
p: X_{I, \mathfrak{D}}^{o} \rightarrow \bar{X}_{I, \nu(\mathfrak{D})}^{o}
$$

over Spec $\mathbb{k}[P] / I$.

Proof. Recall that

$$
\begin{aligned}
R_{\rho, I} & =\frac{R_{I}\left[X_{i-1}, X_{i}^{ \pm}, X_{i+1}\right]}{\left(X_{i-1} X_{i+1}-z^{\eta\left(\left[D_{i}\right]\right)} X_{i}^{-D_{i}^{2}} g_{\rho_{i}} \prod_{j=1}^{\ell_{i}}\left(1+b_{i j} X_{i}^{-1}\right)\right)} \\
\bar{R}_{\rho, I} & =\frac{R_{I}\left[\bar{X}_{i-1}, \bar{X}_{i}^{ \pm}, \bar{X}_{i+1}\right]}{\left(\bar{X}_{i-1} \bar{X}_{i+1}-z^{\eta\left(p^{*}\left[\bar{D}_{i}\right]\right)} \bar{X}_{i}^{-\bar{D}_{i}^{2}} \bar{g}_{\rho_{i}} \prod_{j=1}^{\ell_{i}}\left(1+b_{i j}^{-1} \bar{X}_{i}\right)\right)}
\end{aligned}
$$


We simply define $p_{i}$ to be the identity on $R_{I}$ and $p_{i}\left(X_{j}\right)=\bar{X}_{j}$. This makes sense since $D_{i}^{2}=\bar{D}_{i}^{2}-\ell_{i}$ and $\left[D_{i}\right]=p^{*}\left[\bar{D}_{i}\right]-\sum_{j=1}^{\ell_{i}} E_{i j}$, so that

$$
\begin{aligned}
p_{i}\left(z^{\eta\left(\left[D_{i}\right]\right)} X_{i}^{-D_{i}^{2}} \prod_{j=1}^{\ell_{i}}\left(1+b_{i j} X_{i}^{-1}\right)\right) & =z^{\eta\left(p^{*}\left[\bar{D}_{i}\right]\right)} \bar{X}_{i}^{-\bar{D}_{i}^{2}}\left(\prod_{j=1}^{\ell_{i}} b_{i j}^{-1} \bar{X}_{i}\right)\left(\prod_{j=1}^{\ell_{i}}\left(1+b_{i j} \bar{X}_{i}^{-1}\right)\right) \\
& =z^{\eta\left(p^{*}\left[\bar{D}_{i}\right]\right)} \bar{X}_{i}^{-\bar{D}_{i}^{2}} \prod_{j=1}^{\ell_{i}}\left(1+b_{i j}^{-1} \bar{X}_{i}\right) .
\end{aligned}
$$

The map $p_{i-1, i}$ is induced by $\tilde{\nu}_{\sigma_{i-1, i}}$ defined in (3.7). It is then straightforward to check the commutativity of the three diagrams.

Lemma 3.28. Given Assumptions [3.20, suppose $\nu(\mathfrak{D})$ is a scattering diagram in the sense of Definition 3.21. For $Q \in \sigma_{i-1, i}$, we distinguish between

$$
\operatorname{Lift}_{Q}(q) \in R_{\sigma_{i-1, i}, I}
$$

for the lift of $q \in B_{0}(\mathbb{Z})$ and

$$
\operatorname{Lift}_{\nu(Q)}(\nu(q)) \in \bar{R}_{\bar{\sigma}_{i-1, i}, I}
$$

the lift of $\nu(q)$. Then

(1) $p_{i-1, i}\left(\operatorname{Lift}_{Q}(q)\right)=\operatorname{Lift}_{\nu(Q)}(\nu(q))$.

(2) Under the natural identifications $\left(P_{\bar{\varphi}_{\tau}}\right)^{\mathrm{gp}}=\left(P_{\bar{\varphi}}\right)^{\mathrm{gp}}$, for $\tau \in \bar{\Sigma} \backslash\{0\}, P_{\bar{\varphi}} \subset P_{\bar{\varphi}_{\tau}}$, and for any broken line $\gamma$ for $q, \operatorname{Mono}(\gamma) \in \mathbb{k}\left[P_{\bar{\varphi}}\right]$.

(3) $\nu$ induces a bijection between broken lines: If $\gamma:(-\infty, 0] \rightarrow B_{0}$ is a broken line in $B_{0}$, then $\nu \circ \gamma$ is a broken line in $\bar{B}_{0}$, and conversely, if $\bar{\gamma}:(-\infty, 0] \rightarrow \overline{\mathcal{P}}$ is a broken line in $\bar{B}_{0}$, then $\nu^{-1} \circ \bar{\gamma}$ is a broken line in $B_{0}$.

Proof. (3) implies (1). For (3), clearly it is enough to compare bending and attached monomials of broken lines near a ray $\rho_{i}$.

Consider a broken line $\gamma$ in $B_{0}$ passing from $\sigma_{i-1, i}$ to $\sigma_{i, i+1}$, and let $c z^{q}$ be the monomial attached to the broken line before it crosses over $\rho_{i}$, so that $q \in P_{\varphi_{\sigma_{i-1, i}}}$. Let $\theta_{\rho_{i}}, \bar{\theta}_{\bar{\rho}_{i}}$ be defined by

$$
\begin{aligned}
& \theta_{\rho_{i}}\left(z^{p}\right):=z^{p} f_{\rho_{i}}^{\langle n, r(p)\rangle} \\
& \bar{\theta}_{\bar{\rho}_{i}}\left(z^{p}\right):=z^{p}\left(\bar{g}_{\rho_{i}} \prod_{j=1}^{\ell_{i}}\left(1+b_{i j}^{-1} \bar{X}_{i}\right)\right)^{\langle\bar{n}, \bar{r}(p)\rangle}
\end{aligned}
$$

where $\left(\bar{\rho}_{i}, \bar{g}_{\rho_{i}}\right) \in \nu(\mathfrak{D})$ is the outgoing ray with support $\bar{\rho}_{i}$. Here $n, \bar{n}$ are primitive cotangent vectors vanishing on tangent vectors to $\rho_{i}, \bar{\rho}_{i}$ and positive on $\sigma_{i-1, i}, \bar{\sigma}_{i-1, i}$ respectively. Then we need to show that

$$
p_{i, i+1}\left(\theta_{\rho_{i}}\left(c z^{q}\right)\right)=\bar{\theta}_{\bar{\rho}_{i}}\left(p_{i-1, i}\left(c z^{q}\right)\right)
$$


to get the correspondence between broken lines.

Note that

$$
p_{i-1, i}\left(X_{i-1}\right)=\bar{X}_{i-1}, \quad p_{i, i-1}\left(X_{i}\right)=\bar{X}_{i}, \quad p_{i, i+1}\left(X_{i}\right)=\bar{X}_{i},
$$

but to compute $p_{i, i+1}\left(X_{i-1}\right)$, we need to use the relation (see Proposition 2.5)

$$
X_{i-1} X_{i+1}=z^{\eta\left(\left[D_{i}\right]\right)} X_{i}^{-D_{i}^{2}}
$$

in $\mathbb{k}\left[P_{\varphi_{\rho_{i}}}\right]$ to write

$$
X_{i-1}=z^{\eta\left(\left[D_{i}\right]\right)} X_{i}^{-D_{i}^{2}} X_{i+1}^{-1} .
$$

On the other hand, one has the relation

$$
\bar{X}_{i-1} \bar{X}_{i+1}=z^{\eta\left(p^{*}\left[\bar{D}_{i}\right]\right)} \bar{X}_{i}^{-\bar{D}_{i}^{2}}
$$

in $\mathbb{k}\left[P_{\bar{\varphi}_{\bar{\rho}}}\right]$, so

$$
\begin{aligned}
p_{i, i+1}\left(X_{i-1}\right) & =z^{\eta\left(\left[D_{i}\right]-p^{*}\left[\bar{D}_{i}\right]\right)} \bar{X}_{i}^{-D_{i}^{2}+\bar{D}_{i}^{2}} \bar{X}_{i-1} \\
& =\bar{X}_{i}^{\ell_{i}} \bar{X}_{i-1} \prod_{j=1}^{\ell_{i}} b_{i j}^{-1} .
\end{aligned}
$$

Thus using Assumptions 3.20 for the form of $f_{\rho_{i}}$, we have

$$
\begin{aligned}
p_{i, i+1}\left(\theta_{\rho_{i}}\left(X_{i-1}\right)\right) & =p_{i, i+1}\left(X_{i-1} f_{\rho_{i}}\right) \\
& =\bar{X}_{i}^{\ell_{i}} \bar{X}_{i-1}\left(\prod_{j=1}^{\ell_{i}} b_{i j}^{-1}\right)\left(\prod_{j=1}^{\ell_{i}}\left(1+b_{i j} \bar{X}_{i}^{-1}\right)\right) \bar{g}_{\rho_{i}} \\
& =\bar{X}_{i-1} \bar{g}_{\rho_{i}} \prod_{j=1}^{\ell_{i}}\left(1+b_{i j}^{-1} \bar{X}_{i}\right) \\
& =\bar{\theta}_{\bar{\rho}_{i}}\left(p_{i-1, i}\left(X_{i-1}\right)\right)
\end{aligned}
$$

as desired. Also,

$$
p_{i, i+1}\left(\theta_{\rho_{i}}\left(X_{i}\right)\right)=\bar{X}_{i}=\bar{\theta}_{\bar{\rho}_{i}}\left(p_{i-1, i}\left(\bar{X}_{i}\right)\right) .
$$

Thus (3.11) holds. This shows (3).

For (2), the statement that $P_{\bar{\varphi}} \subset P_{\bar{\varphi}_{\tau}}$ is obvious. For $q \in \sigma \in \Sigma$, by definition the monomial attached to the first domain of linearity of a broken line for $q$ is $z^{\varphi_{\sigma}(q)}$, which is identified under $\tilde{\nu}_{\sigma}$ with $z^{(\nu(q), \bar{\varphi}(\nu(q)))} \in \mathbb{k}\left[P_{\bar{\varphi}}\right]$. For any $\left(\mathfrak{d}, f_{\mathfrak{d}}\right) \in \nu(\mathfrak{D}), f_{\mathfrak{d}} \in \widehat{\mathbb{k}\left[P_{\bar{\varphi}}\right]}$ by assumption, and hence all monomials associated to broken lines in $\bar{B}_{0}$ lie in $\mathbb{k}\left[P_{\bar{\varphi}}\right]$, hence $(2)$. 
Definition 3.29. Let $\overline{\mathfrak{D}}$ be a scattering diagram in the sense of Definition 3.21 for the pair $P, r: P \rightarrow M$ for some toric monoid $P$. Let $I \subset P$ be an ideal with $\sqrt{I}=\mathfrak{m}_{P}$. We define for $q \in \bar{B}_{0}(\mathbb{Z})$ and $Q \in \bar{B}_{0}$,

$$
\operatorname{Lift}_{Q}(q)=\sum \operatorname{Mono}(\gamma) \in \mathbb{k}[P] / I
$$

where the sum is over all broken lines $\gamma$ for $q$ with endpoint $Q$ in $\bar{B}_{0}$ with respect to the scattering diagram $\overline{\mathfrak{D}}$. One sees easily as in Lemma 2.25 that this is a finite sum.

The last crucial result we need for consistency is the following result of [CPS].

Theorem 3.30. With the assumptions of Definition 3.29, suppose furthermore that $\theta_{\gamma, \overline{\mathfrak{D}}} \equiv 1$ for a loop $\gamma$ around the origin. Fix an ideal $I \subset P$ with $\sqrt{I}=\mathfrak{m}_{P}$ and $q \in \bar{B}_{0}(\mathbb{Z})$. If $Q, Q^{\prime} \in M_{\mathbb{R}} \backslash \operatorname{Supp}\left(\overline{\mathfrak{D}}_{I}\right)$ are general, and $\gamma$ is a path connecting $Q$ and $Q^{\prime}$ for which $\theta_{\gamma, \overline{\mathfrak{D}}_{I}}$ is defined, then

$$
\operatorname{Lift}_{Q^{\prime}}(q)=\theta_{\gamma, \overline{\mathfrak{D}}_{I}}\left(\operatorname{Lift}_{Q}(q)\right)
$$

as elements of $\mathbb{k}[P] / I$.

Proof. This is shown in [CPS] in a rather more general setup. For a version of the argument closer to the current setup, see the proof of Theorem 5.35 of [G11].

Proof of Theorem 3.8. By Lemmas 3.15 and 3.19, we can assume we are in the situation of Assumptions 3.20 with $\mathfrak{D}=\mathfrak{D}^{\text {can }}$. In checking (1) of Definition 2.26 for $\mathfrak{D}^{\text {can }}$ in this situation, we want to check equalities

$$
\operatorname{Lift}_{Q^{\prime}}(q)=\theta_{\gamma, \mathfrak{D}^{\text {can }}}\left(\operatorname{Lift}_{Q}(q)\right)
$$

for $Q, Q^{\prime} \in \sigma_{i-1, i}$. By Lemmas 3.27 and 3.28 , it is sufficient to show that

$$
\operatorname{Lift}_{\nu\left(Q^{\prime}\right)}(\nu(q))=\theta_{\bar{\gamma}, \nu(\mathfrak{D})}\left(\operatorname{Lift}_{\nu(Q)}(\nu(q))\right) .
$$

To check this equality we can compare coefficients of monomials, and given any monomial $z^{p}$ appearing on the left- or right-hand sides, we can apply Theorems 3.25 and 3.30, where we take $P=P_{\bar{\varphi}}, I=\mathfrak{m}_{P_{\bar{\varphi}}}^{k}$ for sufficiently large $k$ so that $p \notin I$. The hypothesis $\theta_{\gamma, \nu(\mathfrak{D})} \equiv 1$ of Theorem 3.30 holds by Theorem 3.25 ,

To show (2) of Definition 2.26, we can take $Q=Q_{-}$and $Q^{\prime}=Q_{+}$on opposite sides of a ray $\rho_{i}$. If $\gamma$ is a short path joining $Q$ and $Q^{\prime}$, we still have (3.12) after inverting $f_{i}$. We have a map

$$
\psi:=\left(\psi_{\bar{\rho}_{i},-}, \psi_{\bar{\rho}_{i},+}\right): \bar{R}_{\bar{\rho}_{i}, I} \rightarrow \bar{R}_{\bar{\sigma}_{i-1, i}, I} \times \bar{R}_{\bar{\sigma}_{i, i+1}, I} .
$$


If $f_{i}=\bar{g}_{\rho_{i}} \prod_{j=1}^{\ell_{i}}\left(1+b_{i j}^{-1} \bar{X}_{i}\right)$ then $\psi$ is given by

$$
\begin{aligned}
\bar{X}_{i-1} & \mapsto\left(z^{\bar{\varphi}_{\bar{\rho}_{i}}\left(v_{i-1}\right)}, f_{i} z^{\bar{\varphi}_{\bar{\rho}_{i}}\left(v_{i-1}\right)}\right), \\
\bar{X}_{i} & \mapsto\left(z^{\bar{\varphi}_{\bar{\rho}_{i}}\left(v_{i}\right)}, z^{\bar{\varphi}_{\bar{\rho}_{i}}\left(v_{i}\right)}\right), \\
\bar{X}_{i+1} & \mapsto\left(f_{i} z^{\bar{\varphi}_{\bar{\rho}_{i}}\left(v_{i+1}\right)}, z^{\bar{\varphi}_{\bar{\rho}_{i}}\left(v_{i+1}\right)}\right) .
\end{aligned}
$$

One checks easily that this map is injective. Furthermore, the image is described as follows. Let $I_{\bar{\rho}_{i}} \subset P_{\bar{\varphi}_{\bar{\rho}_{i}}}$ be the monoid ideal

$$
I_{\bar{\rho}_{i}}=\left\{q \in P_{\bar{\rho}_{i}} \mid q-\varphi_{\bar{\sigma}_{i-1, i}}(r(q)) \in I \text { or } q-\varphi_{\bar{\sigma}_{i, i+1}}(r(q)) \in I\right\} .
$$

Then the image consists of those elements $\left(g_{-}, g_{+}\right)$such that every monomial of $g_{-}$and $g_{+}$has exponent in $P_{\bar{\varphi}_{\bar{\rho}_{i}}} \subset P_{\bar{\varphi}_{\bar{\sigma}_{i-1, i}}}, P_{\bar{\varphi}_{\bar{\sigma}_{i, i+1}}}$, and the images $\bar{g}_{ \pm}$of $g_{ \pm}$in $\left(\mathbb{k}\left[P_{\bar{\rho}_{i}}\right] / I_{\bar{\rho}_{i}}\right)_{f_{i}}$ satisfy $\theta_{\gamma, \nu(\mathfrak{D})}\left(\bar{g}_{-}\right)=\bar{g}_{+}$, where this makes sense as we have localized at $f_{i}$. (See e.g., the proof of Lemma 2.34 in [GS07] for a similar statement.) Thus by (3.12) and Lemma 2.25, (2), there is an $\alpha \in \bar{R}_{\bar{\rho}_{i}, I}$ such that

$$
\psi_{\bar{\rho}_{i},-}(\alpha)=\operatorname{Lift}_{\nu(Q)}(\nu(q)), \quad \psi_{\bar{\rho}_{i},+}(\alpha)=\operatorname{Lift}_{\nu\left(Q^{\prime}\right)}(\nu(q)) .
$$

Thus we may take $\operatorname{Lift}_{\rho_{i}}(q)=p_{i}^{-1}(\alpha)$, and by Lemma 3.28, $\psi_{\rho_{i}, \pm}\left(\operatorname{Lift}_{\rho_{i}}(q)\right)=\operatorname{Lift}_{Q_{ \pm}}(q)$, giving consistency.

3.4. Step V: The proof of Theorem 3.25 and the connection with [GPS09]. Here we derive Theorem 3.25 from the main result of [GPS09]. We will need to review one form of this result, which gives an enumerative interpretation for the output of the Kontsevich-Soibelman lemma.

Fix $M \cong \mathbb{Z}^{2}$ as usual. Suppose we are given positive integers $\ell_{1}, \ldots, \ell_{n}$ and primitive vectors $m_{1}, \ldots, m_{n} \in M$. Let $\ell=\sum_{i=1}^{n} \ell_{i}$ and $Q=M \oplus \mathbb{N}^{\ell}$, with $r: Q \rightarrow M$ the projection. Denote the variables in $\mathbb{k}[Q]$ corresponding to the generators of $\mathbb{N}^{\ell}$ as $t_{i j}$, for $1 \leq i \leq n$ and $1 \leq j \leq \ell_{i}$. Consider the scattering diagram for the data $r: Q \rightarrow M$ (in the sense of Definition 3.21)

$$
\mathfrak{D}=\left\{\left(\mathbb{R}_{\geq 0} m_{i}, \prod_{j=1}^{\ell_{i}}\left(1+t_{i j} z^{m_{i}}\right)\right) \mid 1 \leq i \leq n\right\} .
$$

We wish to interpret $\left(\mathfrak{d}, f_{\mathfrak{d}}\right) \in \operatorname{Scatter}(\mathfrak{D}) \backslash \mathfrak{D}$. Choose a complete fan $\Sigma_{\mathfrak{d}}$ in $M_{\mathbb{R}}$ which contains the rays $\mathbb{R}_{\geq 0} m_{1}, \ldots, \mathbb{R}_{\geq 0} m_{n}$ as well as the ray $\mathfrak{d}$ (which may coincide with one of the other rays). Let $X_{\mathfrak{o}}$ be the corresponding toric surface, and let $D_{1}, \ldots, D_{n}, D_{\text {out }}$ be the divisors corresponding to the above rays. Choose general points $x_{i 1}, \ldots, x_{i \ell_{i}} \in$ $D_{i}$, and let

$$
\nu: \tilde{X}_{\mathfrak{d}} \rightarrow X_{\mathfrak{d}}
$$


be the blow-up of all the points $\left\{x_{i j}\right\}$. Let $\tilde{D}_{1}, \ldots, \tilde{D}_{n}, \tilde{D}_{\text {out }}$ be the proper transforms of the divisors $D_{1}, \ldots, D_{n}, D_{\text {out }}$ and $E_{i j}$ the exceptional curve over $x_{i j}$.

Now introduce the additional data of $\mathbf{P}=\left(\mathbf{P}_{1}, \ldots, \mathbf{P}_{n}\right)$, where $\mathbf{P}_{i}$ denotes a sequence $p_{i 1}, \ldots, p_{i \ell_{i}}$ of $\ell_{i}$ non-negative numbers. We will use the notation $\mathbf{P}_{i}=p_{i 1}+\cdots+p_{i \ell_{i}}$ and call $\mathbf{P}_{i}$ an ordered partition. Define

$$
\left|\mathbf{P}_{i}\right|=\sum_{j=1}^{\ell_{i}} p_{i j}
$$

We shall restrict attention to those $\mathbf{P}$ such that

$$
-\sum_{i=1}^{n}\left|\mathbf{P}_{i}\right| m_{i}=k_{\mathbf{P}} m_{\mathfrak{o}}
$$

where $m_{\mathfrak{d}} \in M$ is a primitive generator of $\mathfrak{d}$ and $k_{\mathbf{P}}$ is a positive integer.

Given this data, consider the class $\beta \in A_{1}\left(X_{\mathfrak{d}}, \mathbb{Z}\right)$ specified by the requirement that, if $D$ is a toric divisor of $X_{\mathfrak{o}}$ with $D \notin\left\{D_{1}, \ldots, D_{n}, D_{\text {out }}\right\}$, then $D \cdot \beta=0$; if $D_{\text {out }} \notin$ $\left\{D_{1}, \ldots, D_{n}\right\}$

$$
D_{i} \cdot \beta=\left|\mathbf{P}_{i}\right|, \quad D_{\text {out }} \cdot \beta=k_{\mathbf{P}}
$$

while if $D_{\text {out }}=D_{j}$ for some $j$, then

$$
D_{i} \cdot \beta= \begin{cases}\left|\mathbf{P}_{i}\right| & i \neq j \\ \left|\mathbf{P}_{i}\right|+k_{\mathbf{P}} & i=j\end{cases}
$$

That such a class exists follows easily from (3.13) and the first part of Lemma 3.32 below. It is also unique. We can then define

$$
\beta_{\mathbf{P}}=\nu^{*}(\beta)-\sum_{i=1}^{p} \sum_{j=1}^{\ell_{i}} p_{i j}\left[E_{i j}\right] \in A_{1}\left(\tilde{X}_{\mathfrak{d}}, \mathbb{Z}\right) .
$$

We define $N_{\mathbf{P}}:=N_{\beta_{\mathbf{P}}}$ as in Definition [3.1, using $(\tilde{Y}, \tilde{D})=\left(\tilde{X}_{\mathfrak{d}}, \tilde{D}\right)$, where $\tilde{D}$ is the proper transform of the toric boundary of $X_{\mathfrak{d}}$, and using $C=\tilde{D}_{\text {out }}$. Then one of the main theorems of GPS09] (see $§ 5.7$ of that paper) states

\section{Theorem 3.31.}

$$
\log f_{\mathfrak{d}}=\sum_{\mathbf{P}} k_{\mathbf{P}} N_{\mathbf{P}} t^{\mathbf{P}} z^{-k_{\mathbf{P}} m_{\mathfrak{d}}}
$$

where the sum is over all $\mathbf{P}$ satisfying (3.13) and $t^{\mathbf{P}}$ denotes the monomial $\prod_{i j} t_{i j}^{p_{i j}}$.

We can adapt this theorem for our purposes as follows. Fix a fan $\Sigma$ in $M_{\mathbb{R}}$ defining a complete non-singular toric surface $\bar{Y}$, with $\bar{D}=D_{1}+\cdots+D_{n}$ the toric boundary. Choose points $x_{i 1}, \ldots, x_{i \ell_{i}} \in D_{i}$, and define a new surface $Y$ as the blow-up $\nu: Y \rightarrow \bar{Y}$ at the points $\left\{x_{i j}\right\}$. Let $E_{i j}$ be the exceptional curve over $x_{i j}$. 
Let $P=\operatorname{NE}(\bar{Y})$; because $\bar{Y}$ is toric, this is a finitely generated monoid with $P^{\times}=$ $\{0\}$. Let $\bar{\varphi}: M_{\mathbb{R}} \rightarrow P_{\mathbb{R}}^{\mathrm{gp}}$ be the $\Sigma$-piecewise linear strictly $P$-convex function given by Lemma 1.13 ,

We will need the following, an immediate corollary of Lemma 1.13, using the notation of that lemma applied to the fan $\Sigma$ for $\bar{Y}$ :

Lemma 3.32. If $\sum_{\rho} a_{\rho} t_{\rho} \in \operatorname{ker} s$, then the corresponding element of $\operatorname{ker} s=A_{1}(\bar{Y}, \mathbb{Z})$ is

$$
\sum_{\rho} a_{\rho} \bar{\varphi}\left(m_{\rho}\right) \in P^{\mathrm{gp}}
$$

Let $E \subset A_{1}(Y, \mathbb{Z})$ be the lattice spanned by the classes of the exceptional curves of $\nu$, so that $A_{1}(Y, \mathbb{Z})=\nu^{*} A_{1}(\bar{Y}, \mathbb{Z}) \oplus E$. We then obtain a map

$$
\varphi=\nu^{*} \circ \bar{\varphi}: M_{\mathbb{R}} \rightarrow \nu^{*} P^{g p} \oplus E .
$$

Let

$$
Q=\left\{(m, p) \in M \oplus A_{1}(Y, \mathbb{Z}) \mid \exists p^{\prime} \in \nu^{*} P \oplus E \text { such that } p=p^{\prime}+\varphi(m)\right\} .
$$

There is an obvious projection $r: Q \rightarrow M$, and by strict convexity of $\bar{\varphi}, Q^{\times}=E$.

We consider the scattering diagram, $\overline{\mathfrak{D}}_{0}$, over $\widehat{\mathbb{k}[Q]}$ given by

$$
\overline{\mathfrak{D}}_{0}=\left\{\left(\mathbb{R}_{\geq 0} m_{i}, \prod_{j=1}^{\ell_{i}}\left(1+z^{\left(m_{i}, \varphi\left(m_{i}\right)-E_{i j}\right)}\right)\right) \mid 1 \leq i \leq n\right\} .
$$

Then we have

Theorem 3.33. Let $\left(\mathfrak{d}, f_{\mathfrak{o}}\right) \in \operatorname{Scatter}\left(\overline{\mathfrak{D}}_{0}\right) \backslash \overline{\mathfrak{D}}_{0}$, assuming that there is at most one ray of $\operatorname{Scatter}\left(\overline{\mathfrak{D}}_{0}\right) \backslash \overline{\mathfrak{D}}_{0}$ in each possible outgoing direction. (Note by definition of Scatter $\left(\overline{\mathfrak{D}}_{0}\right),\left(\mathfrak{d}, f_{\mathfrak{d}}\right)$ cannot be incoming. $)$ Then, following the notation of Definition 3.1 and 3.3 ,

$$
\log f_{\mathfrak{o}}=\sum_{\beta} k_{\beta} N_{\beta} z^{\left(-k_{\beta} m_{\mathfrak{⿰}}, \pi_{*}(\beta)-\varphi\left(k_{\beta} m_{\mathfrak{o}}\right)\right)} .
$$

Here $\pi: \tilde{Y} \rightarrow Y$ is the toric blow-up of $Y$ determined by $\mathfrak{d}$ and $C \subset \tilde{Y}$ is the component of the boundary determined by $\mathfrak{d}$. If $\mathfrak{d}$ is not one of the rays $\mathbb{R}_{\geq 0} m_{i}$, then we sum over all $\mathbb{A}^{1}$-classes $\beta \in A_{1}(\tilde{Y}, \mathbb{Z})$ satisfying (3.1), and if $\mathfrak{d}=\mathbb{R}_{\geq 0} m_{i}$ we sum over all such classes except for classes given by multiple covers of one of the exceptional divisors $E_{i j}$. 
Proof. Let $Q^{\prime}$ be the submonoid of $M \oplus \mathbb{N}^{\ell}$ generated by elements of the form $\left(m_{i}, d_{i j}\right)$, where $d_{i j}$ is the $(i, j)$-th generator of $\mathbb{N}^{\ell}$. Note that $Q^{\prime}$ itself is freely generated by these elements. Thus we can define a map

$$
\alpha: Q^{\prime} \rightarrow Q
$$

by $\left(m_{i}, d_{i j}\right) \mapsto\left(m_{i}, \varphi\left(m_{i}\right)-E_{i j}\right)$. The scattering diagram

$$
\mathfrak{D}^{\prime}:=\left\{\left(\mathbb{R}_{\geq 0} m_{i}, \prod_{j=1}^{\ell_{i}}\left(1+z^{\left(m_{i}, d_{i j}\right)}\right)\right) \mid 1 \leq i \leq n\right\}
$$

then has image under the map $\alpha$ (applying $\alpha$ to each $f_{\mathfrak{0}}$ ) the scattering diagram $\overline{\mathfrak{D}}_{0}$. Thus if we apply $\alpha$ to each element of $\operatorname{Scatter}\left(\mathfrak{D}^{\prime}\right)$, we must get $\operatorname{Scatter}\left(\overline{\mathfrak{D}}_{0}\right)$,

as $\theta_{\gamma, \operatorname{Scatter}\left(\mathfrak{D}^{\prime}\right)}$ being the identity on $\widehat{\mathbb{k}\left[Q^{\prime}\right]}$ implies that $\theta_{\gamma, \alpha\left(\operatorname{Scatter}\left(\mathfrak{D}^{\prime}\right)\right)}$ is the identity on $\widehat{\mathbb{k}[Q]}$.

To obtain the result, we now note that the set of possible $\mathbb{A}^{1}$-classes in $\tilde{Y}$ occurring in the expression (3.15) are precisely the classes $\left\{\beta_{\mathbf{P}}\right\}$ where $\mathbf{P}$ runs over all partitions satisfying (3.13). Now applying $\alpha$ to a term appearing in (3.14) of the form

$$
k_{\mathbf{P}} N_{\mathbf{P}} t^{\mathbf{P}} z^{-k_{\mathbf{P}} m_{0}}=k_{\beta_{\mathbf{P}}} N_{\beta_{\mathbf{P}}}\left(\prod t_{i j}^{p_{i j}}\right) z^{\sum_{i=1}^{p}\left|\mathbf{P}_{i}\right| m_{i}},
$$

we get

$$
k_{\beta_{\mathbf{P}}} N_{\beta_{\mathbf{P}}} z^{\left(-k_{\mathbf{P}} m_{0}, \sum_{i=1}^{p}\left|\mathbf{P}_{i}\right| \varphi\left(m_{i}\right)-\sum_{i, j} p_{i j} E_{i j}\right)} .
$$

But by Lemma 3.32 and (3.13),

$$
\sum_{i=1}^{p}\left|\mathbf{P}_{i}\right| \varphi\left(m_{i}\right)-\sum_{i, j} p_{i j} E_{i j}=\pi_{*}\left(\beta_{\mathbf{P}}\right)-\varphi\left(k_{\mathbf{P}} m_{\mathfrak{o}}\right)
$$

hence the result.

A direct comparison of the formula of the above theorem and the formula in the definition of the canonical scattering diagram then yields Theorem 3.25 .

\section{Smoothness: Around the Gross-Siebert locus}

Next we prove that our deformation of $\mathbb{V}_{n}$ is indeed a smoothing. The main theorem of this section (Theorem 4.6) will show this in the situation of Theorem 0.1 when $(Y, D)$ has a toric model. The full smoothness statement of Theorem 0.1 will require some more work, which will be carried out in $\$ 6$.

We prove smoothness by working over the Gross-Siebert locus (Definition 3.14). Here our deformation (when restricted to one-parameter subgroups associated to $p^{*} A$, $A$ an ample divisor on $\bar{Y}$ ) agrees with the construction of [GS07]. This is important here because the deformations of GS07] come with explicit charts that cover all of $\mathbb{V}_{n}$, from which it is clear that they give a smoothing. So conceptually, the smoothing 
claim is clear. Because we work with formal families the actual argument is a bit more delicate. First we make rigorous the notion of a smooth generic fibre for a formal family:

Definition-Lemma 4.1. Let $f: Z \rightarrow W$ be a flat finite type morphism of schemes of relative dimension $d$. Then $\operatorname{Sing}(f) \subset Z$ is the closed embedding defined by the $d^{\text {th }}$ Fitting ideal of $\Omega_{Z / W}^{1}$. Sing $(f)$ is empty if and only if $f$ is smooth. Formation of $\operatorname{Sing}(f)$ commutes with all base extensions of $W$.

Proof. For the definition of the Fitting ideal, see e.g., [E95, 20.4. The fact that it commutes with base-change follows from the fact that $\Omega_{Z / W}^{1}$ commutes with basechange and [E95], Cor. 20.5. That $\operatorname{Sing}(f)$ is empty if and only if $f$ is smooth follows from [E95], Prop. 20.6 and the definition of smoothness.

Now for a formal family, smoothness of the generic fibre is measured by the fact that Sing $(f)$ does not surject scheme-theoretically onto the base. More precisely:

Definition 4.2. Let $S$ be a normal variety, $V \subset S$ a connected closed subset, and $\mathfrak{S}$ the formal completion of $S$ along $V$. Let $\mathfrak{f}: \mathfrak{X} \rightarrow \mathfrak{S}$ be an adic flat morphism of formal schemes of pure relative dimension and $\mathfrak{Z} \subset \mathfrak{X}$ the scheme theoretic singular locus of $\mathfrak{f}$. Then we say the generic fiber of $\mathfrak{f}$ is smooth if the map $\mathcal{O}_{\mathfrak{S}} \rightarrow \mathfrak{f}_{*} \mathcal{O}_{\mathfrak{Z}}$ is not injective.

For the statement of Proposition 4.3, we fix our usual setting of a surface $(Y, D)$, and assume given Assumptions 3.20 and that $\nu(\mathfrak{D})$ is a scattering diagram in the sense of Definition 3.21. Suppose furthermore that $\theta_{\gamma, \nu(\mathcal{D})} \equiv 1$ for a loop $\gamma$ around the origin. Thus by Theorem 3.30, $\mathfrak{D}$ is consistent. These hypotheses on $\mathfrak{D}$ apply in particular when $\mathfrak{D}=\mathfrak{D}^{\text {can }}$.

Let $T^{\mathrm{gs}}$ be the Gross-Siebert locus; we have $T^{\mathrm{gs}}=\operatorname{Spec} \mathbb{k}[P] / G$. Consistency of $\mathfrak{D}$ gives a flat family

$$
f_{I}: X_{I} \rightarrow \operatorname{Spec} \mathbb{k}[P] / I
$$

over a thickening of $T^{\mathrm{gs}}$ whenever $\sqrt{I}=G$.

On the other hand, letting $\bar{\Sigma}$ be the fan for $\bar{Y}$ in $\bar{B}=M_{\mathbb{R}}$, we have the piecewise linear function $\bar{\varphi}: \bar{B} \rightarrow P_{\mathbb{R}}^{\mathrm{gp}}$ with $\kappa_{\bar{\rho}, \bar{\varphi}}=p^{*}\left[\bar{D}_{\bar{\rho}}\right]$, as in (3.6). This now determines the Mumford family

$$
\bar{f}_{I}: \bar{X}_{I} \rightarrow \operatorname{Spec} \mathbb{k}[P] / I .
$$

Our goal is to compare these two families. Note that both $X_{G} \rightarrow T^{\mathrm{gs}}$ and $\bar{X}_{G} \rightarrow T^{\mathrm{gs}}$ are the trivial family $\mathbb{V}_{n} \times T^{\mathrm{gs}} \rightarrow T^{\mathrm{gs}}$. Thus either family contains a canonical copy of $T^{\mathrm{gs}}$, i.e., $\{0\} \times T^{\mathrm{gs}}$, where 0 is the vertex of $\mathbb{V}_{n}$. 
Proposition 4.3. In the above situation, fix an ideal I with $\sqrt{I}=G$. There are open affine sets $\bar{U}_{I} \subset \bar{X}_{I}, U_{I} \subset X_{I}$, both sets containing the canonical copy of $T^{\mathrm{gs}}$, and an isomorphism

$$
\mu_{I}: U_{I} \rightarrow \bar{U}_{I}
$$

of families over Spec $\mathbb{k}[P] / I$.

Moreover, there is a non-zero monomial $y \in \mathbb{k}[P]$ whose pullback to $X_{I}$ is in the stalk at any point $x \in\{0\} \times T^{\mathrm{gs}} \subset \mathbb{V}_{n} \times T^{\mathrm{gs}}$ of the ideal of $\operatorname{Sing}_{f_{I}}$ for all $I$.

Proof. The generic fibre of the Mumford family over Spec $\mathbb{k}[P]$ is smooth: indeed the family is trivial over the open torus orbit of $\operatorname{Spec} \mathbb{k}[P]$, with fibre an algebraic torus. It follows that there is a non-zero monomial $y \in \mathbb{k}[P]$ in the ideal of $\operatorname{Sing}_{\bar{f}}$ for the global Mumford family

$$
\bar{f}: \operatorname{Spec} \mathbb{k}\left[P_{\bar{\varphi}}\right] \rightarrow \operatorname{Spec} \mathbb{k}[P] .
$$

Of course its restriction then lies in the stalk at any point $x$ of the ideal sheaf of $\operatorname{Sing}_{\bar{f}_{I}}$ for all $I$. Thus once we establish the claimed isomorphisms, the final statement follows.

Recall from $\$ 3.3$ the construction of $\overline{\mathfrak{D}}:=\nu(\mathfrak{D})$ and the scheme $\bar{X}_{I, \overline{\mathfrak{D}}}^{o}$ from $\overline{\mathfrak{D}}$. By Lemma 3.27, $X_{I, \mathfrak{D}}^{o} \cong \bar{X}_{I, \mathfrak{D}}^{o}$, so we in fact have an isomorphism

$$
X_{I} \cong \operatorname{Spec} \Gamma\left(\bar{X}_{I, \overline{\mathfrak{D}}}^{o}, \mathcal{O}_{\bar{X}_{I, \overline{\mathfrak{D}}}^{o}}\right)=: \bar{X}_{I, \overline{\mathfrak{D}}}
$$

So we can work with $\bar{X}_{I, \overline{\mathfrak{D}}}$ instead of $X_{I}$. On the other hand, the Mumford family Spec $\mathbb{k}\left[P_{\bar{\varphi}}\right] / I \mathbb{k}\left[P_{\bar{\varphi}}\right]$ over $\operatorname{Spec} \mathbb{k}[P] / I$ can be described similarly. Using the empty scattering diagram instead of the scattering diagram $\overline{\mathfrak{D}}$, one has by Lemma 2.9

$$
\bar{X}_{I, \emptyset}=\operatorname{Spec} \Gamma\left(\bar{X}_{I, \emptyset}^{o}, \mathcal{O}_{\bar{X}_{I, \emptyset}^{o}}\right) .
$$

Now define an ideal $I_{0} \subset P_{\bar{\varphi}}$ as follows. For $\sigma \in \bar{\Sigma}_{\text {max }}$, let $\bar{\varphi}_{\sigma}$ denote the linear extension of $\left.\bar{\varphi}\right|_{\sigma}$. We set

$$
I_{0}:=\left\{(m, p) \in P_{\bar{\varphi}} \mid p-\bar{\varphi}_{\sigma}(m) \in I \text { for some } \sigma \in \bar{\Sigma}_{\max }\right\} .
$$

Note that $\sqrt{I_{0}}=\mathfrak{m}_{P_{\bar{\varphi}}}$. By assumption, $\overline{\mathfrak{D}}$ is a scattering diagram for $P_{\bar{\varphi}}$, and hence there are only a finite number of $\left(\mathfrak{d}, f_{\mathfrak{d}}\right) \in \overline{\mathfrak{D}}$ for which $f_{\mathfrak{d}} \not \equiv 1 \bmod I_{0}$. Furthermore, modulo $I_{0}$, each $f_{\mathfrak{o}}$ is a polynomial.

Let $\overline{\mathfrak{D}}_{I}$ be the scattering diagram obtained from $\overline{\mathfrak{D}}$ by, for each outgoing ray $\left(\mathfrak{d}, f_{\mathfrak{d}}\right)$, truncating each $f_{\mathfrak{o}}$ by throwing out all terms which lie in $I_{0}$. The incoming rays remain unchanged. Thus $\overline{\mathfrak{D}}_{I}$ can be viewed as a finite scattering diagram. Let

$$
h:=\prod_{\mathfrak{d} \in \overline{\mathfrak{D}}_{I}} f_{\mathfrak{d}} .
$$

This is an element of $\mathbb{k}\left[P_{\bar{\varphi}}\right]$. Note that necessarily $h \equiv 1 \bmod \mathfrak{m}_{P_{\bar{\varphi}}}$. Thus $h \neq 0$ defines an open subset $\bar{U} \subset \operatorname{Spec} \mathbb{k}\left[P_{\bar{\varphi}}\right] / G \mathbb{k}\left[P_{\bar{\varphi}}\right]=\bar{X}_{G, \bar{D}}=\bar{X}_{G, \emptyset}=\mathbb{V}_{n} \times T^{\mathrm{gs}}$. Furthermore, 
$\bar{U}$ contains the canonical copy of $T^{\mathrm{gs}}$. Since $\bar{X}_{I, \overline{\mathfrak{D}}}$ and $\bar{X}_{I, \emptyset}$ both have underlying topological space $\bar{X}_{G}$, this defines open sets $\bar{U}_{I, \overline{\mathcal{D}}}$ of $\bar{X}_{I, \overline{\mathfrak{D}}}$ and $\bar{U}_{I, \emptyset}$ of $\bar{X}_{I, \emptyset}$. We shall show these two open subschemes are isomorphic.

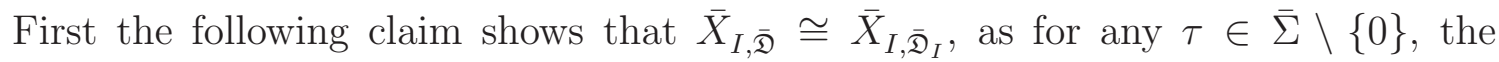
automorphisms involved will have the same effect modulo $I_{\tau}$. As a consequence, we can work with the scattering diagram $\overline{\mathfrak{D}}_{I}$.

Claim 4.4. Let $\tau \in \bar{\Sigma}$, and suppose $(m, p) \in P_{\bar{\varphi}}$ satisfies $-m \in \tau$. Then $(m, p) \in I_{0}$ if and only if $(m, p) \in I_{\tau}$, where

$$
I_{\tau}:=\left\{(m, p) \in P_{\bar{\varphi}_{\tau}} \mid p-\bar{\varphi}_{\sigma}(m) \in I \text { for some } \sigma \in \bar{\Sigma}_{\max } \text { with } \tau \subset \sigma\right\} .
$$

Proof of claim. Clearly $I_{\tau} \cap P_{\bar{\varphi}} \subset I_{0}$, so one implication is clear. Conversely, suppose that $(m, p) \in I_{0}$, so that $p-\bar{\varphi}_{\sigma}(m) \in I$ for some $\sigma \in \Sigma_{\max }$. If $\tau \subset \sigma^{\prime} \in \Sigma_{\max }$, let $\rho_{1}, \ldots, \rho_{n}$ be the sequence of rays traversed in passing from $\sigma$ to $\sigma^{\prime}$, chosen so that all $\rho_{1}, \ldots, \rho_{n}$ lie in a half-plane bounded by the line $\mathbb{R} m$. Then

$$
\bar{\varphi}_{\sigma^{\prime}}(m)=\bar{\varphi}_{\sigma}(m)+\sum_{i=1}^{n}\left\langle n_{\rho_{i}}, m\right\rangle \kappa_{\rho_{i}, \bar{\varphi}},
$$

with $n_{\rho_{i}}$ primitive, vanishing on $\rho_{i}$, and positive on $\rho_{i+1}$. Note that since $-m \in \tau$, we must have $\left\langle n_{\rho_{i}}, m\right\rangle \leq 0$ for each $i$, and hence $p-\bar{\varphi}_{\sigma^{\prime}}(m)=p-\bar{\varphi}_{\sigma}(m)+p^{\prime}$ for some $p^{\prime} \in P$. Hence $(m, p) \in I_{\tau}$.

To show that $\bar{U}_{I, \overline{\mathfrak{D}}}$ and $\bar{U}_{I, \emptyset}$ are isomorphic, let us describe these open subschemes explicitly away from the origin. Recall that $\bar{X}_{I, \mathfrak{D}}^{o}$ is obtained by gluing together schemes which are spectra of rings $\bar{R}_{\tau, I}$ for $\tau \in \bar{\Sigma}$. However in the case that $\operatorname{dim} \tau=1$, this ring depends on the scattering diagram, so we write $\bar{R}_{\tau, I, \mathfrak{D}}$ for $\mathfrak{D}=\overline{\mathfrak{D}}$ or $\emptyset$.

If $\operatorname{dim} \tau=2$, then $\bar{R}_{\tau, I, \mathfrak{D}}=\mathbb{k}\left[P_{\bar{\varphi}_{\tau}}\right] / I \mathbb{k}\left[P_{\bar{\varphi}_{\tau}}\right]$. Since $h \in \mathbb{k}\left[P_{\bar{\varphi}}\right] \subset \mathbb{k}\left[P_{\bar{\varphi}_{\tau}}\right], h$ defines an element of $\bar{R}_{\tau, I, \mathfrak{D}}$ in this case.

If $\operatorname{dim} \tau=1$, then $\tau=\rho_{i}$ for some $i$, and we have a surjection

$$
\bar{R}_{\rho_{i}, I, \mathcal{O}} \rightarrow \bar{R}_{\rho_{i}, G, \mathcal{Q}}=R_{G}\left[\bar{X}_{i-1}, \bar{X}_{i}^{ \pm 1}, \bar{X}_{i+1}\right] /\left(\bar{X}_{i-1} \bar{X}_{i+1}\right) \cong \mathbb{k}\left[P_{\bar{\varphi}_{\rho_{i}}}\right] / G \mathbb{k}\left[P_{\bar{\varphi}_{\rho_{i}}}\right],
$$

so that $h \in \mathbb{k}\left[P_{\bar{\varphi}}\right] \subset \mathbb{k}\left[P_{\bar{\varphi}_{\rho_{i}}}\right]$ defines an element of $\bar{R}_{\rho_{i}, G, \mathcal{D}}$. Choosing any lift of $h$ to $\bar{R}_{\rho_{i}, I, \mathfrak{D}}$, we note the localization $\left(\bar{R}_{\rho_{i}, I, \mathfrak{D}}\right)_{h}$ is independent of the lift since the kernel of the above surjection is nilpotent.

We can then define regardless of $\operatorname{dim} \tau$,

$$
S_{\tau, I, \mathfrak{D}}:=\left(\bar{R}_{\tau, I, \mathfrak{D}}\right)_{h} .
$$

Note there is an isomorphism

$$
\psi_{i}: S_{\rho_{i}, I, \overline{\mathfrak{D}}} \rightarrow S_{\rho_{i}, I, \emptyset}
$$


given by

$$
\bar{X}_{i-1} \mapsto \bar{X}_{i-1}\left(\bar{g}_{\rho_{i}} \prod_{j=1}^{\ell_{i}}\left(1+b_{i j}^{-1} \bar{X}_{i}\right)\right), \quad \bar{X}_{i} \mapsto \bar{X}_{i}, \quad \bar{X}_{i+1} \mapsto \bar{X}_{i+1}
$$

This has an inverse because of the localization at $h$.

Given a path $\gamma$ in $M_{\mathbb{R}} \backslash\{0\}$, note that by construction of $h, \theta_{\gamma, \overline{\mathfrak{D}}_{I}}$ makes sense as an automorphism of the localization $\mathbb{k}\left[P_{\bar{\varphi}}\right]_{h}$, since to define the automorphism associated with crossing a ray $\left(\mathfrak{d}, f_{\mathfrak{d}}\right)$, we only need $f_{\mathfrak{d}}$ to be invertible. However since by construction $h$ is divisible by $f_{\mathfrak{d}}, f_{\mathfrak{d}}$ is invertible. In particular, $\theta_{\gamma, \overline{\mathfrak{D}}_{I}}$ also makes sense as an automorphism of $\left(\mathbb{k}\left[P_{\bar{\varphi}_{\tau}}\right] / I \mathbb{k}\left[P_{\bar{\varphi}_{\tau}}\right]\right)_{h}=S_{\tau, I, \emptyset}$ for any $\tau \in \bar{\Sigma} \backslash\{0\}$. Thus using the equality $S_{\sigma, I, \emptyset}=S_{\sigma, I, \overline{\mathfrak{D}}}$ for $\operatorname{dim} \sigma=2$ we see that $\theta_{\gamma, \overline{\mathfrak{D}}_{I}}$ also makes sense as an automorphism of $S_{\sigma, I, \overline{\mathfrak{D}}}$.

Choose an orientation on $M_{\mathbb{R}}$, labelling the rays $\rho_{1}, \ldots, \rho_{n}$ of $\bar{\Sigma}$ in a counterclockwise order, with $\sigma_{i-1, i}$ as usual the maximal cone containing $\rho_{i-1}$ and $\rho_{i}$. For two distinct points $p, q$ on the unit circle in $M_{\mathbb{R}}$ not contained in $\operatorname{Supp}\left(\overline{\mathfrak{D}}_{I}\right)$, let $\gamma_{p, q}$ be a counterclockwise path from $p$ to $q$, and write $\theta_{p, q}$ for $\theta_{\gamma_{p, q}, \overline{\mathfrak{D}}_{I}}$ acting on any of the rings $S_{\tau, I, \emptyset}$.

For each $\rho_{i}$, let $p_{i,+}$ be a point on this unit circle contained in the connected component of $\sigma_{i, i+1} \backslash \operatorname{Supp}\left(\overline{\mathfrak{D}}_{I}\right)$ adjacent to $\rho_{i}$, and $p_{i,-}$ a point in this unit circle contained in the connected component of $\sigma_{i-1, i} \backslash \operatorname{Supp}\left(\overline{\mathfrak{D}}_{I}\right)$ adjacent to $\rho_{i}$.

Choose a base-point $q$ on the unit circle not in $\operatorname{Supp}\left(\overline{\mathfrak{D}}_{I}\right)$.

Recall in the construction of $\bar{X}_{I, \mathfrak{D}}^{o}$, the open sets $\operatorname{Spec} \bar{R}_{\rho_{i+1}, I, \mathfrak{D}}$ and $\operatorname{Spec} \bar{R}_{\rho_{i}, I, \mathfrak{D}}$ are glued together along the common open set $\operatorname{Spec} \bar{R}_{\sigma_{i, i+1}, I, \mathfrak{D}}$ using the trivial automorphism or the automorphism $\theta_{p_{i,+}, p_{i+1,-}}$ in the cases $\mathfrak{D}=\emptyset$ or $\mathfrak{D}=\overline{\mathfrak{D}}$ respectively. After localizing at $h$, we have a commutative diagram

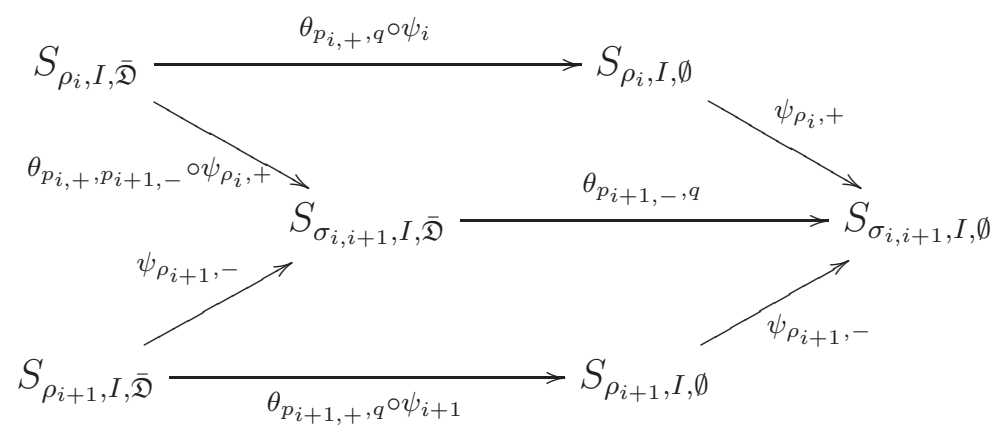

Here the maps $\psi_{\rho, \pm}$ are the ones defined in Proposition 2.5 and (2.8). This shows that the isomorphisms $\theta_{p_{i,+}} \circ \psi_{i}$ between Spec $S_{\rho_{i}, I, \bar{D}}$ and Spec $S_{\rho_{i}, I, \emptyset}$ are compatible with the gluings, and hence give an isomorphism between $\bar{U}_{I, \mathfrak{D}} \backslash\left(\{0\} \times T^{\mathrm{gs}}\right)$ and $\bar{U}_{I, \emptyset} \backslash\left(\{0\} \times T^{\mathrm{gs}}\right)$. 
Now $\mathbb{V}_{n}$ satisfies Serre's condition $S_{2}$. Since $\bar{X}_{I}$ and $X_{I}$ are flat deformations of $\mathbb{V}_{n} \times T^{\mathrm{gs}}$, by Lemma 2.10 the above isomorphism extends across the codimension two set $\{0\} \times T^{\mathrm{gs}}$, giving the desired isomorphism between $U_{I}$ and $\bar{U}_{I}$.

We now need to use the above observations along the Gross-Siebert locus to obtain results about deformations away from the Gross-Siebert locus. For the remainder of the section, we work with data $(Y, D), \eta, P$, but now as in Assumptions 3.13, Furthermore, we take $\mathfrak{D}=\mathfrak{D}^{\text {can }}$. Thus if we take $I$ an ideal with either $\sqrt{I}=G$ or $\sqrt{I}=\mathfrak{m}$, we obtain a flat family $X_{I} \rightarrow \operatorname{Spec} R_{I}=: S_{I}$, and in the former case, $X_{I} \rightarrow S_{I}$ restricts to the open subscheme of Spec $R_{I}$ whose underlying open subset is $T^{\mathrm{gs}}$, giving the family over the thickening of the Gross-Siebert locus.

With $J=\mathfrak{m}$ or $G$, let $\mathfrak{f}_{J}: \mathfrak{X}_{J} \rightarrow \mathfrak{S}_{J}$ denote the formal deformation determined by the deformations $X_{J^{N+1}} \rightarrow \operatorname{Spec} R_{J^{N+1}}$ for $N \geq 0$. Thus $\mathfrak{S}_{J}=\operatorname{Spf}\left(\lim _{\longleftarrow} \mathbb{k}[P] / J^{N+1}\right)$ is the formal spectrum of the $J$-adic completion of $\mathbb{k}[P], \mathfrak{X}_{J}$ is a formal scheme, and $\mathfrak{X}_{J} \rightarrow \mathfrak{S}_{J}$ is an adic flat morphism of formal schemes. We refer to [G60] for background on formal schemes.

Let $Z_{I}:=\operatorname{Sing}\left(f_{I}\right) \subset X_{I}$ denote the singular locus of $f_{I}: X_{I} \rightarrow S_{I}$. Thus $Z_{I} \subset X_{I}$ is a closed embedding of schemes. Since the singular locus is compatible with basechange, the singular loci $Z_{J^{n}} \subset X_{J^{n}}$ determine a closed embedding $\mathfrak{Z}_{J} \subset \mathfrak{X}_{J}$ which we refer to as the singular locus of $\mathfrak{f}_{J}: \mathfrak{X}_{J} \rightarrow \mathfrak{S}_{J}$.

Again, with $J=\mathfrak{m}$ or $G$, we have a section $s: S_{J} \rightarrow X_{J}=S_{J} \times \mathbb{V}_{n}$ given by $s(t)=t \times\{0\}$ for $t \in S_{J}$. We write $X_{J}^{o}:=X_{J} \backslash s\left(S_{J}\right) \subset X_{J}$ and $X_{I}^{o} \subset X_{I}, \mathfrak{X}_{J}^{o} \subset \mathfrak{X}_{J}$ for the induced open embeddings.

Lemma 4.5. In the above situation, there exists $0 \neq g \in \mathbb{k}[P]$ such that $\operatorname{Supp}\left(g \cdot \mathcal{O}_{\mathfrak{Z}_{J}}\right)$ is contained in $s\left(S_{J}\right)$. In particular, $\mathfrak{f}_{J *}\left(g \cdot \mathcal{O}_{\mathfrak{Z}_{J}}\right)$ is a coherent sheaf on $\mathfrak{S}_{J}$.

Proof. We can write an explicit open covering $\left\{\mathfrak{U}_{i, J}\right\}$ of $\mathfrak{X}_{J}^{o}$ in the two cases $J=\mathfrak{m}$ or $J=G$, as follows. Write $a_{i}=z^{\left[D_{i}\right]}$ and $m_{i}=-D_{i}^{2}$. In the case $J=\mathfrak{m}$,

$$
\mathfrak{U}_{i, J}=V\left(X_{i-1} X_{i+1}-a_{i} X_{i}^{m_{i}}\right) \subset \mathbb{A}_{X_{i-1}, X_{i+1}}^{2} \times\left(\mathbb{G}_{m}\right)_{X_{i}} \times \mathfrak{S}_{J} .
$$

In the case $J=G$,

$$
\mathfrak{U}_{i, J}=V\left(X_{i-1} X_{i+1}-a_{i} X_{i}^{m_{i}} \prod\left(1+b_{i j} X_{i}^{-1}\right)\right) \subset \mathbb{A}_{X_{i-1}, X_{i+1}}^{2} \times\left(\mathbb{G}_{m}\right)_{X_{i}} \times \mathfrak{S}_{J},
$$

with $b_{i j}=z^{\left[E_{i j}\right]}$ as usual.

We now use the charts $\mathfrak{U}_{i, J}$ to compute the singular locus explicitly. In the case $J=\mathfrak{m}$, the singular locus $\mathfrak{Z}_{i, J}$ of $\mathfrak{U}_{i, J} / \mathfrak{S}_{J}$ is given by

$$
\mathfrak{Z}_{i, J}=V\left(X_{i-1}, X_{i+1}, a_{i}\right) \subset \mathfrak{U}_{i, J} .
$$

Hence if we define $g=a_{1} \cdots a_{n}$ then $\operatorname{Supp}\left(g \cdot \mathcal{O}_{\mathfrak{Z}_{J}}\right)$ is contained in $s\left(S_{J}\right)$. 
Similarly, if $J=G$, the structure sheaf of the singular locus of $\mathfrak{U}_{i, J}$ is annihilated by $g_{i}:=a_{i} \prod_{j \neq k}\left(b_{i j}-b_{i k}\right)$. (Here $\prod_{j \neq k}\left(b_{i j}-b_{i k}\right)$ is the discriminant of the polynomial $f\left(X_{i}\right):=\prod\left(X_{i}+b_{i j}\right)$. It is a linear combination of $f$ and $f^{\prime}$ with coefficients in $\mathbb{k}\left[\left\{b_{i j}\right\}\right]\left[X_{i}\right]$. See [L02], p. 200-204.) So we can take $g=g_{1} \cdots g_{n}$.

The support of $g \cdot \mathcal{O}_{\mathfrak{Z}_{J}}$ is a closed subset of $s\left(S_{J}\right)$, hence proper over $S_{J}$. It follows that $\mathfrak{f}_{J *}\left(g \cdot \mathcal{O}_{\mathfrak{Z}_{J}}\right)$ is coherent by [G61], 3.4.2.

Theorem 4.6. Let $(Y, D), \eta, P$ satisfy Assumptions 3.13, and take $\mathfrak{D}=\mathfrak{D}^{\text {can }}$. Then the maps $\mathbb{k}[P] \rightarrow \mathfrak{f}_{\mathfrak{m}_{*}} \mathcal{O}_{\mathfrak{Z}_{\mathfrak{m}}}$ and $\mathcal{O}_{\mathfrak{S}_{\mathfrak{m}}} \rightarrow \mathfrak{f}_{\mathfrak{m}_{*}} \mathcal{O}_{\mathfrak{Z}_{\mathfrak{m}}}$ are not injective, so the generic fibre of $\mathfrak{f}_{\mathfrak{m}}$ is smooth in the sense of Definition 4.2. This also implies that for $I=\mathfrak{m}^{N+1}$ and $N \gg 0$, the map $\mathbb{k}[P] / I \rightarrow f_{I *} \mathcal{O}_{\operatorname{Sing}\left(f_{I}\right)}$ is not injective.

Proof. By Lemma 4.5 there exists $0 \neq g \in \mathbb{k}[P]$ such that $\operatorname{Supp}\left(g \cdot \mathcal{O}_{\mathfrak{Z}_{G}}\right) \subset s\left(S_{G}\right)$. Let $E$ be the subgroup of $P^{g p}$ generated by $P \backslash G$, so that $U=\operatorname{Spec} \mathbb{k}[P+E]$ is an open subset of Spec $\mathbb{k}[P]$. Denote by $\mathfrak{S}_{G}^{o}$ the open subset of $\mathfrak{S}_{G}$ isomorphic to the completion of $U$ along the subscheme defined by $G+E$. This is the formal thickening of the Gross-Siebert locus $T^{\mathrm{gs}}$. By Proposition 4.3 there then exists $0 \neq h \in \mathbb{k}[P+E]$ such that $\operatorname{Supp}\left(h \cdot \mathcal{O}_{\mathfrak{Z}_{G} \cap \mathfrak{f}_{G}^{-1}\left(\mathfrak{S}_{G}^{o}\right)}\right)$ is disjoint from $s\left(S_{G} \cap U\right)$. By multiplying $h$ by a monomial whose exponent lies in $P \backslash G$, we can assume that $h \in \mathbb{k}[P]$. Thus $g h \cdot \mathcal{O}_{\mathfrak{Z}_{G}}$ has support in the closed subset $s\left(S_{G} \backslash\left(S_{G} \cap U\right)\right)$. Since this sheaf is coherent, there exists a non-zero element $k \in \mathfrak{m} \subset \mathbb{k}[P]$ such that $g h k \cdot \mathcal{O}_{\mathfrak{Z}_{G}}=0$. Noting by construction that $g h k \in \mathbb{k}[P]$, we have $\mathbb{k}[P] \rightarrow \Gamma\left(\mathfrak{Z}_{G}, \mathcal{O}_{\mathfrak{Z}_{G}}\right)$ is not injective, hence the composition $\mathbb{k}[P] \rightarrow \Gamma\left(\mathfrak{Z}_{G}, \mathcal{O}_{\mathfrak{Z}_{G}}\right) \rightarrow \Gamma\left(\mathfrak{Z}_{\mathfrak{m}}, \mathcal{O}_{\mathfrak{Z}_{\mathfrak{m}}}\right)$ is not injective. Since $\mathbb{k}[P] \subset \Gamma\left(\mathfrak{S}_{\mathfrak{m}}, \mathcal{O}_{\mathfrak{S}_{\mathfrak{m}}}\right), \mathcal{O}_{\mathfrak{S}_{\mathfrak{m}}} \rightarrow$ $\mathfrak{f}_{\mathfrak{m} *} \mathcal{O}_{\mathfrak{Z} \mathfrak{m}}$ is not injective.

\section{The Relative torus}

The flat deformations $X_{I, \mathfrak{D}^{\text {can }}} \rightarrow \operatorname{Spec} \mathbb{k}[P] / I$ produced by the canonical scattering diagram have a useful special property: there is a natural torus action on the total space $X_{I, \mathfrak{D}^{\text {can }}}$ compatible with a torus action on the base. The meaning of this action will be clarified in Part II, where we will prove that our family extends naturally, in the positive case, to a universal family of Looijenga pairs $(Z, D)$ together with a choice of isomorphism $D \stackrel{\sim}{\rightarrow} D_{*}$, where $D_{*}$ is a fixed $n$-cycle. The torus action then corresponds to changing the choice of isomorphism.

Fixing the pair $(Y, D)$ as usual, $D=D_{1}+\cdots+D_{n}$, let $\mathbb{A}^{D}=\mathbb{A}^{n}$ be the affine space with one coordinate for each component $D_{i}$. Let $T^{D}$ be the diagonal torus acting on $\mathbb{A}^{D}$, i.e., the torus $T^{D}$ whose character group

$$
\chi\left(T^{D}\right)=\mathbb{Z}^{D}
$$

is the free module with basis $e_{D_{1}}, \ldots, e_{D_{n}}$. 
Definition 5.1. We define a canonical map $w: A_{1}(Y) \rightarrow \chi\left(T^{D}\right)$ given by

$$
C \mapsto \sum\left(C \cdot D_{i}\right) e_{D_{i}}
$$

Suppose $P \subset A_{1}(Y)$ is a toric submonoid containing $\mathrm{NE}(Y)$. We then get an action of $T^{D}$ on $\operatorname{Spec} \mathbb{k}[P]$, as well as on $\operatorname{Spec} \mathbb{k}[P] / I$ for any monomial ideal $I$, and hence also on $\operatorname{Spf}(\widehat{\mathbb{k}[P]})$ for any completion of $\mathbb{k}[P]$ with respect to a monomial ideal.

We can also define a unique piecewise linear map

$$
w: B \rightarrow \chi\left(T^{D}\right) \otimes_{\mathbb{Z}} \mathbb{R}
$$

with $w(0)=0$ and $w\left(v_{i}\right)=e_{D_{i}}$, for $v_{i}$ the primitive generator of the ray $\rho_{i}$.

Theorem 5.2. Let $I$ be an ideal for which $X_{I, \mathfrak{D} \text { can }} \rightarrow \operatorname{Spec} \mathbb{k}[P] / I$ is defined. Then $T^{D}$ acts equivariantly on $X_{I, \mathfrak{D} \text { can }} \rightarrow$ Spec $\mathbb{k}[P] / I$. Furthermore, each theta function $\vartheta_{q}$, $q \in B(\mathbb{Z})$, is an eigenfunction of this action, with character $w(q)$.

Proof. It's enough to check this on the open subset $X_{I, \mathfrak{D}^{\text {can }}}^{o} \subset X_{I, \mathfrak{D} \text { can }}$. We have a cover of $X_{I, \mathfrak{D}^{\text {can }}}^{o}$ by open sets the hypersurfaces

$$
U_{\rho_{i}, I} \subset \mathbb{A}_{X_{i-1}, X_{i+1}}^{2} \times\left(\mathbb{G}_{m}\right)_{X_{i}} \times \operatorname{Spec} R_{I}
$$

given by the equation

$$
X_{i-1} X_{i+1}=z^{\left[D_{i}\right]} X_{i}^{-D_{i}^{2}} f_{\rho_{i}}
$$

where $f_{\rho_{i}}$ is the function attached to the ray $\rho_{i}$ in $\mathfrak{D}^{\text {can }}$. If we act on $X_{j}$ with weight $w\left(v_{j}\right)$ and on $z^{p}$ with weight $w(p)$ (for $p \in P$ ), then we note that for every $\left(\mathfrak{d}, f_{\mathfrak{d}}\right) \in \mathfrak{D}^{\text {can }}$, every monomial in $f_{\mathfrak{d}}$ has weight zero by the explicit description of $f_{\mathfrak{d}}$ in Definition 3.3 . In particular, the equation defining $U_{\rho_{i}, I}$ is clearly $T^{D}$-equivariant, and each of the monomials is an eigenfunction.

Now $X_{I, \mathfrak{D} \text { can }}^{o}$ is obtained by gluing $U_{\rho_{i}, \sigma_{i, i+1}, I} \subset U_{\rho_{i}, I}$ with $U_{\rho_{i+1}, \sigma_{i, i+1}, I} \subset U_{\rho_{i+1}, I}$, using scattering automorphisms of $\mathfrak{D}^{\text {can }}$, and these open sets are naturally identified with $\left(\mathbb{G}_{m}\right)_{X_{i}, X_{i+1}}^{2} \times \operatorname{Spec} R_{I}$. The scattering automorphisms commute with the $T^{D}$ action, by the fact that the scattering functions have weight zero. Thus $T^{D}$ acts equivariantly on $X_{I, \mathfrak{D} \text { can }} \rightarrow \operatorname{Spec} \mathbb{k}[P] / I$.

Now we check our canonical global function $\vartheta_{q}$ is an eigenfunction, with character $w(q)$. By construction, given a broken line $\gamma$, the weights of monomials attached to adjacent domains of linearity are the same, since the functions in the scattering diagram are of weight zero. Thus the weight of $\operatorname{Mono}(\gamma)$ only depends on $q$. This weight can be determined by fixing the base point $Q$ in a cone $\sigma$ which contains $q$, in which case the broken line for $q$ which doesn't bend and is wholly contained in $\sigma$ yields the monomial $z^{\varphi_{\sigma}(q)}$, which has weight $w(q)$. Thus $\vartheta_{q}$ is an eigenfunction with weight $w(q)$. 


\section{EXTENDING THE FAMILY OVER BOUNDARY STRATA}

Here we prove Theorem 0.1 and Theorem 0.2 . Let us review what we know so far. For any pair $(Y, D)$, we know that $\mathfrak{D}^{\text {can }}$ is consistent by Theorem 3.8. Thus, if the number $n$ of irreducible components of $D$ satisfies $n \geq 3$, Theorem 2.28 gives the construction of $f: X_{I} \rightarrow \operatorname{Spec} R_{I}$ of Theorem 0.1. The algebra structure on $A_{I}$ has structure constants given by counts of broken lines as in Theorem 2.34. The $T^{D}$ equivariance is given by Theorem 5.2. If furthermore $(Y, D)$ has a toric model, then the smoothness statement follows from Theorem 4.6.

We will give a proof of Theorem 0.2 and the remaining cases of 0.1 by first proving Theorem 0.2 in the case that we know that we have the desired algebra structure on $A_{I}$, and then bootstrap to the general case for both theorems.

6.1. Theorem $\mathbf{0 . 2}$ in the case that $(Y, D)$ has a toric model. As usual, let $P$ be the toric monoid associated to a strictly convex rational polyhedral cone $\sigma_{P} \subset A_{1}(Y)_{\mathbb{R}}$ which contains the Mori cone $\mathrm{NE}(Y)_{\mathbb{R}}$. We have $\mathfrak{m}=P \backslash\{0\}$. For a monomial ideal $I \subset P$ we define

$$
A_{I}:=\bigoplus_{q \in B(\mathbb{Z})} R_{I} \cdot \vartheta_{q}
$$

where $R_{I}=\mathbb{k}[P] / I$. We take throughout $\mathfrak{D}=\mathfrak{D}^{\text {can }}$.

Assumptions 6.1. For any monomial ideal $I$ with $\sqrt{I}=\mathfrak{m}$, the multiplication rule of Theorem 2.34 defines an $R_{I}$-algebra structure on $A_{I}$, so that $A_{I} \otimes_{R_{I}} R_{\mathfrak{m}}=H^{0}\left(\mathbb{V}_{n}, \mathcal{O}_{\mathbb{V}_{n}}\right)$.

Note we have already shown that Assumptions 6.1 hold if $n \geq 3$ by Theorems 2.28, 2.34 and 3.8 .

Let $\Gamma \subset B(\mathbb{Z})$ be a finite collection of integral points such that the corresponding functions $\vartheta_{q}$ generate the $\mathbb{k}$-algebra $H^{0}\left(\mathbb{V}_{n}, \mathcal{O}_{\mathbb{V}_{n}}\right)$. (Then the $\vartheta_{q}, q \in \Gamma$ generate $A_{I}$ as an $R_{I}$-algebra if $\sqrt{I}=\mathfrak{m}$ and Assumptions 6.1 hold.) Note for $n \geq 3$ we can take for $\Gamma$ the points $\left\{v_{i}\right\}$, and for $n=1,2$, one can make a simple choice for $\Gamma$, see 6.2 .

Lemma 6.2. For any monomial ideal $J \subset P, \bigcap_{k>0}\left(J+\mathfrak{m}^{k}\right)=J$.

Proof. The inclusion $\supset$ is obvious. For the other direction, as the intersection is a monomial ideal, it's enough to consider a monomial in the intersection. But notice that a monomial is in $J+\mathfrak{m}^{k}$ iff it is either in $J$ or in $\mathfrak{m}^{k}$. The result follows since $\bigcap \mathfrak{m}^{k}=0$.

Assuming 6.1, let $\mathcal{A}$ be the collection of monomial ideals $J \subset P$ with the following properties:

(1) There is an $R_{J}$-algebra structure on $A_{J}$ such that the canonical isomorphism of $R$-modules $A_{J} \otimes_{R_{J}} R_{I+J}=A_{I+J}$ is an algebra isomorphism, for all $\sqrt{I}=\mathfrak{m}$. 
(2) $\vartheta_{q}, q \in \Gamma$ generate $A_{J}$ as an $R_{J}$-algebra.

By the lemma, the algebra structure in (1) is unique if it exists. The algebra structure on all $A_{I}$ determines such a structure on $\widehat{A}:=\lim _{\sqrt{I}=\mathfrak{m}} A_{I}, \widehat{A}_{J}:=\lim _{\sqrt{I}=\mathfrak{m}} A_{I+J}$. Also, there are canonical inclusions

$$
\begin{gathered}
\widehat{A} \subset \prod_{q \in B(\mathbb{Z})} \hat{R} \cdot \vartheta_{q} \\
\widehat{A}_{J} \subset \prod_{q \in B(\mathbb{Z})} \hat{R}_{J} \cdot \vartheta_{q}
\end{gathered}
$$

where $\hat{R}$ is the completion of $R$ at $\mathfrak{m}$ and $\hat{R}_{J}=\lim _{\longleftarrow} R /(I+J)$ the inverse limit over all ideals $I$ with $\sqrt{I}=\mathfrak{m}$. Here the direct products are viewed purely as $\hat{R}, \hat{R}_{J}$ modules. We can also view

$$
A_{J}:=\bigoplus_{q \in B(\mathbb{Z})} R_{J} \cdot \vartheta_{q} \subset \prod_{q \in B(\mathbb{Z})} \hat{R}_{J} \cdot \vartheta_{q} .
$$

It is clear that $A_{J} \subset \widehat{A}_{J}$ (as submodules of the direct product). Thus (1) holds if and only if the following holds:

$\left(1^{\prime}\right)$ For each $p, q \in B(\mathbb{Z})$, at most finitely many $z^{C} \vartheta_{s}$ with $C \notin J$ appear in the product expansion of Theorem 2.34 for $\vartheta_{p} \cdot \vartheta_{q} \in \widehat{A}_{J}$.

Lemma 6.3. If $J \in \mathcal{A}$ and $J \subset J^{\prime}$, then $J^{\prime} \in \mathcal{A}$. In addition, $\mathcal{A}$ is closed under finite intersections.

Proof. The first statement is clear. Now assume $J_{1}, J_{2} \in \mathcal{A}$. It's clear that $\left(1^{\prime}\right)$ holds for $J_{1} \cap J_{2}$, so $A_{J_{1} \cap J_{2}}$ is an algebra. Moreover we have an exact sequence of $\mathbb{k}$-modules

$$
0 \rightarrow A_{J_{1} \cap J_{2}} \rightarrow A_{J_{1}} \times A_{J_{2}} \rightarrow A_{J_{1}+J_{2}} \rightarrow 0
$$

exhibiting $A_{J_{1} \cap J_{2}}$ as the fibre product $A_{J_{1}} \times_{A_{J_{1}+J_{2}}} A_{J_{2}}=: A_{1} \times_{B} A_{2}=: A$. We now show this fibre product is a finitely generated $\mathbb{k}$-algebra. Indeed, note that since the maps $A_{1}, A_{2} \rightarrow B$ are surjective, so are the maps $A \rightarrow A_{i}$. Let $\left\{u_{i}\right\}$ be a generating set for the ideal $\operatorname{ker}\left(A_{2} \rightarrow B\right)$. Since $A_{i}$ is Noetherian, one can find a finite such set. Note that $\tilde{u}_{i}:=\left(0, u_{i}\right) \in A$. In addition, choose finite sets $\left\{x_{i}\right\},\left\{y_{j}\right\}$ generating $A_{1}$ and $A_{2}$ as $\mathbb{k}$-algebras. For each of these elements, choose a lift to $A$, giving a finite set of lifts $\left\{\tilde{u}_{i}, \tilde{x}_{i}, \tilde{y}_{i}\right\}$, which we claim generate $A$. Indeed, given $(x, y) \in A$, one can subtract a polynomial in the $\tilde{x}_{i}$ 's to obtain $\left(0, y^{\prime}\right)$. Necessarily $y^{\prime} \in \operatorname{ker}\left(A_{2} \rightarrow B\right)$, and hence we can write $y^{\prime}=\sum f_{i} u_{i}$ with $f_{i}$ a polynomial in the $y_{i}$ 's. Let $\tilde{f}_{i}$ be the same polynomial in the $\tilde{y}_{i}{ }^{\prime}$ s. Then $\sum \tilde{f}_{i} \tilde{u}_{i}=\left(0, y^{\prime}\right)$, showing generation.

Thus $A_{J_{1} \cap J_{2}}$ is also a finitely generated $R_{J_{1} \cap J_{2}}$-algebra. Now the generation statement follows from Lemma 6.4 taking $R=R_{J_{1} \cap J_{2}}, S=A_{J_{1} \cap J_{2}}, I=J_{1} / J_{1} \cap J_{2}, J=J_{2} / J_{1} \cap J_{2}$, $\Gamma=\left\{q_{1}, \ldots, q_{m}\right\}$, and the map $R\left[T_{1}, \ldots, T_{m}\right] \rightarrow S$ given by $T_{i} \mapsto \vartheta_{q_{i}}$. 
Lemma 6.4. Let $I, J \subset R$ be ideals in a Noetherian ring, with $I \cdot J=0$, and let $S$ be a finitely generated $R$-algebra, and $R\left[T_{1}, \ldots, T_{m}\right] \rightarrow S$ an $R$-algebra map which is surjective modulo $I$ and $J$. Then the map is surjective.

Proof. The associated map $\operatorname{Spec} S \rightarrow \mathbb{A}^{m} \times \operatorname{Spec} R$ is proper, as can be easily checked using the valuative criterion for properness. Indeed, any map $S \rightarrow K$ for a field $K$ factors through either $S / I S$ or $S / J S$. Since this is a map of affine schemes, $S$ is a finite $R\left[T_{1}, \ldots, T_{m}\right]$-module. Now we can apply Nakayama's lemma.

Proposition 6.5. There is a unique minimal radical monomial ideal $I_{\min } \subset P$ such that (1) and (2) hold for any monomial ideal $J$ with $I_{\min } \subset \sqrt{J}$.

Proof. Certainly any ideal $J$ with $\sqrt{J}=\mathfrak{m}$ lies in $\mathcal{A}$. Note that a radical monomial ideal is the complement of a union of faces of $P$, so there are only a finite number of such ideals. Suppose $I_{1}, I_{2}$ are two radical ideals such that $J_{i} \in \mathcal{A}$ for any $J_{i}$ with $I_{i} \subset \sqrt{J_{i}}$. Note that any ideal $J$ with $I_{1} \cap I_{2} \subset \sqrt{J}$ can be written as $J_{1} \cap J_{2}$, with $I_{i} \subset \sqrt{J_{i}}$. (Indeed, we can use the primary decomposition of $J$. If $J=\bigcap_{k} \mathfrak{p}_{k}$ is an intersection of primary ideals, necessarily the prime ideal $\sqrt{\mathfrak{p}_{k}}$ contains either $I_{1}$ or $I_{2}$ for each $k$. Then let $J_{1}$ be the intersection of those $\mathfrak{p}_{k}$ whose radical contains $I_{1}$ and $J_{2}$ be the intersection of those $\mathfrak{p}_{k}$ whose radical contains $I_{2}$.) Thus by Lemma 6.3. $J \in \mathcal{A}$. This shows the existence of $I_{\min }$.

Proposition 6.6. Assume 6.1.

(1) Suppose the intersection matrix $\left(D_{i} \cdot D_{j}\right)$ is not negative semi-definite. Then $I_{\min }=(0) \subset \mathbb{k}[P]$.

(2) Suppose $F \subset \sigma_{P}$ is a face such that $F$ does not contain the class of every component of $D$. Then $I_{\min } \subset P \backslash F$.

Proof. We prove both cases simultaneously, writing $F:=P$ in case (1). We claim there exists an effective divisor $W=\sum a_{i} D_{i}$ with support $D$ such that $W \cdot D_{j}>0$ for all $D_{j}$ contained in $F$ and $a_{i}>0$ for all $i$. For case (1), see Lemma 6.9, In case (2), say $\left[D_{1}\right] \notin F$. Then we can take $a_{1} \gg a_{2} \gg \cdots \gg a_{n}>0$.

The algebra structure depends only on the deformation type of $(Y, D)$. By Proposition 4.1 of GHK12, we may replace $(Y, D)$ by a deformation equivalent pair such that any irreducible curve $C \subset Y$ intersects $D$.

Let $\overline{\mathrm{NE}}(Y)_{\mathbb{R}} \subset A_{1}(Y, \mathbb{R})$ denote the closure of $\mathrm{NE}(Y)_{\mathbb{R}}$. Let $F^{\prime}:=\overline{\mathrm{NE}}(Y)_{\mathbb{R}} \cap F$, a face of $\overline{\mathrm{NE}}(Y)_{\mathbb{R}}$. Define $\Delta=D-\epsilon W, 0<\epsilon \ll 1$. Then $(Y, \Delta)$ is KLT (Kawamata log terminal).

We claim $K_{Y}+\Delta \sim-\epsilon W$ is negative on $F^{\prime} \backslash\{0\}$. By construction $\left(K_{Y}+\Delta\right) \cdot D_{j}<0$ for $\left[D_{j}\right] \in F^{\prime}$ and $\left(K_{Y}+\Delta\right) \cdot C<0$ for $C \not \subset D$. Let $N$ be a nef divisor such that 
$F^{\prime}=\overline{\mathrm{NE}}(Y)_{\mathbb{R}} \cap N^{\perp}$. Then $a N-\left(K_{Y}+\Delta\right)$ is nef and big for $a \gg 0$, and thus some multiple of $N$ defines a birational morphism $g$ by the basepoint-free theorem [KM98, Theorem 3.3. Thus $F^{\prime}$ is generated by exceptional curves of $g$. We deduce that $\left(K_{Y}+\Delta\right)^{\perp} \cap F^{\prime}=\{0\}$ and $\left(K_{Y}+\Delta\right)$ is negative on $F^{\prime} \backslash\{0\}$ as claimed.

Now by the cone theorem [KM98, Theorem 3.7, $\overline{\mathrm{NE}}(Y)_{\mathbb{R}}$ is rational polyhedral near $F^{\prime}$ and there is a contraction $p: Y \rightarrow \bar{Y}$ such that $F^{\prime}$ is generated by the classes of curves contracted by $p$. It follows that we can find $\mathrm{NE}(Y)_{\mathbb{R}} \subset \sigma_{P^{\prime}} \subset \sigma_{P}$ such that $F^{\prime}$ is a face of $\sigma_{P^{\prime}}$. Now the algebra structure for $P$ comes from $P^{\prime}$ by base extension, so (replacing $P$ by $P^{\prime}$ ) we can assume $F=F^{\prime}$, and thus that $W$ is positive on $F \backslash\{0\}$.

Now let $J$ be a monomial ideal with $\sqrt{J}=P \backslash F$. Consider condition $\left(1^{\prime}\right)$. By the $T^{D}$-equivariance of Theorem [5.2, any $z^{C} \vartheta_{s}$ that appears in $\vartheta_{p} \cdot \vartheta_{q}$ has the same weight for $T^{D}$. Thus it is enough to show that the map

$$
w: B(\mathbb{Z}) \times(P \backslash J) \rightarrow \chi\left(T^{D}\right), \quad(q, C) \mapsto w(q)+w(C)
$$

has finite fibres. It is enough to consider fibres of $\sigma(\mathbb{Z}) \times(P \backslash J) \rightarrow \chi\left(T^{D}\right)$ for each $\sigma \in \Sigma_{\max }$. Note that $\sigma(\mathbb{Z}) \times P$ is the set of integral points of a rational polyhedral cone, and $w$ is linear on this set. Thus it is enough to check that $\operatorname{ker}(w) \cap(\sigma(\mathbb{Z}) \times F)=0$. So suppose we have $q \in \sigma(\mathbb{Z}), C \in F$ with $w(q)+w(C)=0$. Say $\sigma=\sigma_{i, i+1}$. Then $q=a v_{i}+b v_{i+1}$, for $a, b \in \mathbb{Z}_{\geq 0}$. We have

$$
w(q)+w(C)=a e_{D_{i}}+b e_{D_{i+1}}+\sum_{j}\left(C \cdot D_{j}\right) e_{D_{j}} ;
$$

thus if this is zero, we have $C \cdot D_{j} \leq 0$ for all $j$. In particular, $W \cdot C \leq 0$. Since $W$ is positive on $F \backslash\{0\}, C=0$. Now necessarily $a=b=q=0$. This proves $\left(1^{\prime}\right)$.

For (2), let $A_{J}^{\prime} \subset A_{J}$ be the subalgebra generated by the $\vartheta_{q}, q \in \Gamma$. Fix a weight $w \in \chi\left(T^{D}\right)$. To show $A_{J}^{\prime}=A_{J}$ it is enough to show that the finite set

$$
\left\{z^{C} \vartheta_{q} \in A_{J} \mid(q, C) \in B(\mathbb{Z}) \times(P \backslash J) \text { of weight } w\right\}
$$

is contained in $A_{J}^{\prime}$ (since the $z^{C} \vartheta_{q}$ give a $\mathbb{k}$-basis of $A_{J}$ ). We argue by decreasing induction on $\operatorname{ord}_{\mathfrak{m}}(C)$ (see Definition 2.21). Since the set of possible $(q, C)$ is finite, there is an upper bound on the possible $\operatorname{ord}_{\mathfrak{m}}$ 's. So the claim is vacuously true for large $\operatorname{ord}_{\mathfrak{m}}$. Consider $z^{C} \cdot \vartheta_{p}$, with $\operatorname{ord}_{\mathfrak{m}}(C)=h$. Since the $\vartheta_{q}$ generate $A_{J}$ modulo $\mathfrak{m}$, we can find $a \in A_{J}^{\prime}$ such that

$$
\vartheta_{p}=a+m
$$

with $m \in \mathfrak{m} \cdot A_{J}$. Moreover, we can assume $a$, and thus $m$, is homogeneous for the $T^{D}$ action. Now

$$
z^{C} \vartheta_{p}=z^{C} a+z^{C} m
$$


Clearly $z^{C} m$ is a sum of terms $z^{D} \vartheta_{q}$ of weight $w$ and $\operatorname{ord}_{\mathfrak{m}}(D)>h$, so $z^{C} m \in A_{J}^{\prime}$ by induction.

Remark 6.7. Suppose $p: Y \rightarrow Y^{\prime}$ is a contraction such that some component of $D$ is not contracted by $p$. Let $F$ be the face of $\mathrm{NE}(Y)_{\mathbb{R}}$ generated by classes of curves contracted by $p$. Then $\mathrm{NE}(Y)_{\mathbb{R}}$ is rational polyhedral near $F$. (This follows from the cone theorem, cf. the proof of Proposition 6.6.) In particular there exists a rational polyhedral cone $\sigma_{P} \subset A_{1}(Y, \mathbb{R})$ such that $\mathrm{NE}(Y)_{\mathbb{R}} \subset \sigma_{P}$ and $\sigma_{P}$ coincides with $\mathrm{NE}(Y)_{\mathbb{R}}$ near $F$.

Corollary 6.8. Theorem 0.2 holds if $D$ has $n \geq 3$ irreducible components.

Proof. Immediate from Proposition 6.6.

6.2. Proof of Theorems $\mathbf{0 . 1}$ and $\mathbf{0 . 2}$ in general. We now consider an arbitrary Looijenga pair $(Y, D)$, along with a toric monoid $P$ with $\mathrm{NE}(Y) \subset P \subset A_{1}(Y, \mathbb{Z})$. Let $\tau:\left(Y^{\prime}, D^{\prime}\right) \rightarrow(Y, D)$ be a toric blowup such that $\left(Y^{\prime}, D^{\prime}\right)$ has a toric model $p:\left(Y^{\prime}, D^{\prime}\right) \rightarrow(\bar{Y}, \bar{D})$. We have the map $\tau_{*}: A_{1}\left(Y^{\prime}, \mathbb{Z}\right) \rightarrow A_{1}(Y, \mathbb{Z})$. We can find a strictly convex rational polyhedral cone $\sigma_{P^{\prime}}$ with

$$
\mathrm{NE}\left(Y^{\prime}\right)_{\mathbb{R}} \subset \sigma_{P^{\prime}} \subset A_{1}\left(Y^{\prime}, \mathbb{R}\right)
$$

which has a face $F$ spanned by the $\tau$-exceptional curves, and which surjects under $\tau_{*}$ onto $\sigma_{P} \subset A_{1}(Y, \mathbb{R})$. For any monomial ideal $I \subset P$ with $\sqrt{I}=\mathfrak{m}$, let $I^{\prime} \subset P^{\prime}$ be the inverse image of $I$ under $\tau_{*}$. Then $\sqrt{I^{\prime}}$ is the prime monomial ideal associated to the face $F$. Since the exceptional curves are a proper subset of $D^{\prime}$ we have $\sqrt{I^{\prime}} \in$ $\mathcal{A}\left(Y^{\prime}\right)$ by Proposition 6.6. Note that Spec $\mathbb{k}[P] / I$ is naturally a closed subscheme of Spec $\mathbb{k}\left[P^{\prime}\right] / I^{\prime}$, via the map induced by the surjection $\tau_{*}: P^{\prime} \rightarrow P$. Now restrict the family $\mathcal{X}_{I^{\prime}} \rightarrow \operatorname{Spec} \mathbb{k}\left[P^{\prime}\right] / I^{\prime}$ to $\operatorname{Spec} \mathbb{k}[P] / I$. This gives an algebra structure on

$$
A_{I}:=\bigoplus_{q \in B(\mathbb{Z})}(\mathbb{k}[P] / I) \vartheta_{q}
$$

We now verify Assumptions 6.1. First, we show that the multiplication rule of this algebra is the one described in Theorem 2.34. The argument is just as in the proof of Proposition [3.12. We have $B_{\left(Y^{\prime}, D^{\prime}\right)}=B_{(Y, D)}$ and take $\psi:=\tau_{*}: P^{\prime} \rightarrow P$. Note $\psi\left(\mathfrak{D}_{\left(Y^{\prime}, D^{\prime}\right)}^{\text {can }}\right)=\mathfrak{D}_{(Y, D)}^{\text {can }}$ (i.e., the rays are the same, and we apply $\psi$ to the decoration function). This does not literally give a bijection on broken lines (because different exponents in the decoration of a ray in $\mathfrak{D}_{\left(Y^{\prime}, D^{\prime}\right)}^{\text {can }}$ could map to the same exponent under 
$\psi$ ). However, by Equation (3.4), with $z$ a point close to $q$,

$$
\begin{aligned}
\sum_{\substack{\left(\gamma_{1}, \gamma_{2}\right) \\
\operatorname{Limits}\left(\gamma_{i}\right)=\left(q_{i}, z\right) \\
s\left(\gamma_{1}\right)+s\left(\gamma_{2}\right)=q}} c\left(\gamma_{1}\right) c\left(\gamma_{2}\right) & =\sum_{\substack{\operatorname{Limits}\left(\gamma_{i}\right)=\left(\gamma_{i}, z\right) \\
s\left(\gamma_{1}\right)+s\left(\gamma_{2}\right)=q}} \psi\left(\sum_{\gamma_{1}^{\prime} \in \xi_{\gamma_{1}}} c\left(\gamma_{1}^{\prime}\right)\right) \psi\left(\sum_{\gamma_{2}^{\prime} \in \xi_{\gamma_{2}}} c\left(\gamma_{2}^{\prime}\right)\right) \\
& =\psi\left(\sum_{\substack{\left(\gamma_{1}^{\prime}, \gamma_{2}^{\prime}\right) \\
\operatorname{Limits}\left(\gamma_{i}^{\prime}\right)=\left(q_{i}, z\right) \\
s\left(\gamma_{1}^{\prime}\right)+s\left(\gamma_{2}^{\prime}\right)=q}} c\left(\gamma_{1}^{\prime}\right) c\left(\gamma_{2}^{\prime}\right)\right),
\end{aligned}
$$

where $\xi_{\gamma_{i}}$ denotes the set of all broken lines $\gamma_{i}^{\prime}$ for $\mathfrak{D}_{\left(Y^{\prime}, D^{\prime}\right)}^{\text {can }}$ such that $\psi \circ \gamma_{i}^{\prime}=\gamma_{i}$ as paths and the monomials attached to $\psi\left(\gamma_{i}^{\prime}\right)$ differ from those attached to $\gamma_{i}$ only in the $\mathbb{k}$-valued coefficients (see the proof of Proposition 3.12). This implies the claim.

Next we need to check that the fibre over the zero stratum of Spec $\mathbb{k}[P]$ is $\mathbb{V}_{n}$. In case $n \geq 3$, this is straightforward from the multiplication rule. Indeed, modulo $\mathfrak{m}$, every broken line contributing to the multiplication rule is a straight line, and furthermore it cannot cross any ray of $\Sigma$. ¿From this one sees that $A_{\mathfrak{m}}=R_{\mathfrak{m}}[\Sigma]$.

The cases $n=1$ and 2 require special attention. We will do the case of $n=1$, as $n=2$ is similar (and simpler). We cut $B=B_{(Y, D)}$ along the unique ray $\rho=$ $\rho_{1} \in \Sigma$, and consider the image under a set of linear coordinates $\psi$ on $B \backslash \rho$. This identifies $B \backslash \rho$ with a strictly convex rational cone in $\mathbb{R}^{2}$. Let $w, w^{\prime}$ be the primitive generators of the two boundary rays. Modulo $\mathfrak{m}$ the decoration on every scattering ray is trivial, so every broken line is straight. Moreover, no line can cross $\rho$ (or the attached monomial becomes trivial modulo $\mathfrak{m}$ by the strict convexity of $\varphi$ ). Now it follows for any $x \in B(\mathbb{R}) \backslash \rho$ and any $q \in(B \backslash \rho)(\mathbb{Z})$ there is a unique (straight) broken line with Limits $=(q, x)$, while there are exactly two (straight) broken lines with Limits $=(v, x), v=v_{1}$ - under $\psi$ these become two distinct straight lines with directions $w, w^{\prime}$. Performing a toric blowup of $(Y, D)$ to get $n^{\prime}=3$ can be accomplished by subdividing the cone generated by $w$ and $w^{\prime}$ along the rays generated by $w+w^{\prime}$ and $2 w+w^{\prime}$. Then by Theorem 0.2 in the case $n=3$, we see that $A_{\mathfrak{m}}$ is generated over $\mathbb{k}$ by

$$
\vartheta_{v}=\vartheta_{w}=\vartheta_{w^{\prime}}, \vartheta_{w+w^{\prime}}, \vartheta_{2 w+w^{\prime}}
$$

where we abuse notation and use the same symbol for an integer point in the convex cone generated by $w$ and $w^{\prime}$, and the corresponding point in $B(\mathbb{Z})$. Now applying the 
multiplication rule of Theorem 2.34 one checks easily the equalities:

$$
\begin{aligned}
\vartheta_{v} \cdot \vartheta_{w+w^{\prime}} & =\vartheta_{2 w+w^{\prime}}+\vartheta_{w+2 w^{\prime}} \\
\vartheta_{2 w+w^{\prime}} \cdot \vartheta_{w+2 w^{\prime}} & =\vartheta_{3 w+3 w^{\prime}}=\vartheta_{w+w^{\prime}}^{3}
\end{aligned}
$$

It follows that

$$
\vartheta_{2 w+w^{\prime}} \cdot \vartheta_{v} \cdot \vartheta_{w+w^{\prime}}=\vartheta_{2 w+w^{\prime}}^{2}+\vartheta_{w+w^{\prime}}^{3}
$$

and thus $A_{\mathfrak{m}}=\mathbb{k}[x, y, z] /\left(x y z-x^{2}-z^{3}\right)$, which is isomorphic to the ring of sections

$$
\bigoplus_{m \geq 0} H^{0}(C, \mathcal{O}(m))
$$

for a line bundle $\mathcal{O}(1)$ of degree one on an irreducible rational nodal curve $C$ of arithmetic genus 1. Thus Spec $A_{\mathfrak{m}}=\mathbb{V}_{1}$.

Combining this with Propositions 6.5 and 6.6, this proves Theorems 0.1 and 0.2 hold for all $(Y, D)$ except for the smoothness statement of Theorem 0.1 .

To show smoothness, note that if $\mathfrak{m}^{\prime}$ denotes the maximal monomial ideal of $P^{\prime}$, $\mathfrak{X}_{\mathfrak{m}^{\prime}}^{\prime} \rightarrow \mathfrak{S}_{\mathfrak{m}^{\prime}}$ the formal deformation provided by Theorem 0.1 for the pair $\left(Y^{\prime}, D^{\prime}\right)$ with the toric model, we know that $\mathbb{k}\left[P^{\prime}\right] \rightarrow H^{0}\left(\mathfrak{Z}_{\mathfrak{m}^{\prime}}, \mathcal{O}_{\mathfrak{Z}_{\mathfrak{m}^{\prime}}}\right)$ is not injective by Theorem 4.6. Now choose (see the beginning of the proof of Proposition 6.6) a divisor $A=$ $\sum_{i} a_{i} D_{i}^{\prime}$ with $a_{i} \geq 0$ for all $i$ and $A$ relatively $\tau$-ample, so that $A \cdot D_{j}^{\prime}>0$ for any $D_{j}^{\prime}$ contracted by $\tau$. This determines a one-parameter subgroup $T^{A} \cong \mathbb{G}_{m}$ of $T^{D^{\prime}}$ via the $\operatorname{map} \chi\left(T^{D^{\prime}}\right) \rightarrow \mathbb{Z}$ given by $e_{D_{i}^{\prime}} \mapsto a_{i}$.

Let $J=P^{\prime} \backslash F$, so that $[C] \in J$ if and only if $C$ is not contracted by $\tau$. Thus if $[C] \in F, T^{A}$ acts on $z^{[C]}$ with weight $C . A>0$, and for $q \in B(\mathbb{Z}), T^{A}$ acts with non-negative weight since $a_{i} \geq 0$ for all $i$. It then follows that the map

$$
H^{0}\left(\mathfrak{Z}_{J}, \mathcal{O}_{\mathfrak{Z}_{J}}\right) \rightarrow H^{0}\left(\mathfrak{Z}_{\mathfrak{m}^{\prime}}, \mathcal{O}_{\mathfrak{Z}_{\mathfrak{m}^{\prime}}}\right)
$$

is injective because every component of $\mathfrak{Z}_{J}$ has a limit point in $\mathfrak{Z}_{\mathfrak{m}^{\prime}}$ under the $T^{A}$ action. So we conclude that $\mathbb{k}\left[P^{\prime}\right] \rightarrow H^{0}\left(\mathfrak{Z}_{J}, \mathcal{O}_{\mathfrak{Z}_{J}}\right)$ is not injective.

Now $F^{\mathrm{gp}}$ is generated by the classes of the $D_{i}^{\prime}$ contracted by $\tau$. Let $T^{F}:=\operatorname{Hom}\left(F, \mathbb{G}_{m}\right)$. The composition $F^{\text {gp }} \subset A_{1}\left(Y^{\prime}, \mathbb{Z}\right) \rightarrow \chi\left(T^{D^{\prime}}\right)$ is a primitive embedding, because the intersection matrix of $F \subset\left\langle D_{1}^{\prime}, \ldots, D_{r}^{\prime}\right\rangle$ is unimodular, where $D_{1}^{\prime}, \ldots, D_{r}^{\prime}$ are the irreducible components of the boundary of $Y^{\prime}$. So the corresponding composition $T^{D^{\prime}} \rightarrow \operatorname{Hom}\left(A_{1}\left(Y^{\prime}, \mathbb{Z}\right), \mathbb{G}_{m}\right) \rightarrow T^{F}$ admits a splitting $T^{F} \rightarrow T^{D^{\prime}}$. By $T^{F}$-equivariance, the restriction of the family $\mathfrak{X}_{J}^{\prime} / \mathfrak{S}_{J}^{\prime}$ to the open subscheme of $\mathfrak{S}_{J}^{\prime}$ defined by $T^{F} \subset S_{J}^{\prime}$ is isomorphic to a direct product of $\mathfrak{X}_{\mathfrak{m}} / \mathfrak{S}_{\mathfrak{m}}$ (coming from $(Y, D), P$ ) with $T^{F}$. In particular, $\mathfrak{X}_{\mathfrak{m}} / \mathfrak{S}_{\mathfrak{m}}$ has smooth generic fibre. 


\subsection{The case that $(Y, D)$ is positive.}

Lemma 6.9. The following are equivalent for a Looijenga pair $(Y, D)$ :

(1.1) There exist integers $a_{1}, \ldots, a_{n}$ such that $\left(\sum a_{i} D_{i}\right)^{2}>0$.

(1.2) There exist positive integers $b_{1}, \ldots, b_{n}$ such that $\left(\sum b_{i} D_{i}\right) \cdot D_{j}>0$ for all $j$.

(1.3) $Y \backslash D$ is the minimal resolution of an affine surface with (at worst) Du Val singularities.

(1.4) There exist $0<c_{i}<1$ such that $-\left(K_{Y}+\sum c_{i} D_{i}\right)$ is nef and big.

If any of the above equivalent conditions hold, then so do the following:

(2.1) The Mori cone $\mathrm{NE}(Y)_{\mathbb{R}}$ is rational polyhedral, generated by finitely many classes of rational curves. Every nef line bundle on $Y$ is semi-ample.

(2.2) The subgroup $G$ of $\operatorname{Aut}(\operatorname{Pic}(Y),\langle\cdot, \cdot\rangle)$ fixing the classes $\left[D_{i}\right]$ is finite.

(2.3) The union $R \subset Y$ of all curves disjoint from $D$ is contractible.

Definition 6.10. We say a Looijenga pair $(Y, D)$ is positive if it satisfies any of the equivalent conditions (1.1)-(1.4) of the above lemma.

Proof. We have

$$
K_{Y}+\sum c_{i} D_{i}=\left(K_{Y}+D\right)-\sum\left(1-c_{i}\right) D_{i}=-\sum\left(1-c_{i}\right) D_{i}
$$

so (1.2) implies (1.4), and (1.2) obviously implies (1.1).

If (1.1) holds then $\left(D^{\perp},\langle\cdot, \cdot\rangle\right)$, where $D^{\perp}=\left\{H \in \operatorname{Pic} Y \mid H \cdot D_{i}=0 \quad \forall i\right\}$, is negative definite, by the Hodge Index Theorem, and this implies (2.2) and (2.3).

Suppose (1.4) holds. By the basepoint-free theorem [KM98, 3.3, the linear system

$$
\left|m\left(\sum b_{i} D_{i}\right)\right|=\left|-m\left(K_{Y}+\sum c_{i} D_{i}\right)\right|
$$

defines a birational morphism for $m \in \mathbb{N}$ sufficiently large, with exceptional locus the union $R$ of curves disjoint from $D$. Adjunction shows $R$ is a contractible configuration of (-2)-curves, which gives (1.3). (2.1) follows from the cone theorem [KM98], 3.7.

We show (1.1) implies (1.2). By the Riemann-Roch theorem, if $W$ is a Weil divisor (on any smooth surface) and $W^{2}>0$ then either $W$ or $-W$ is big (i.e., the rational map given by $|n W|$ is birational for sufficiently large $n$ ). So, possibly replacing the divisor by its negative, we may assume $W=\sum a_{i} D_{i}$ is big. Write

$$
W^{\prime}=\sum_{a_{i}>0} a_{i} D_{i}=W+\sum_{-a_{i}>0}\left(-a_{i}\right) D_{i} .
$$

Thus $W^{\prime}$ is big, and replacing $W$ by $n W^{\prime}$, we may assume all $a_{i} \geq 0$ and $|W|$ defines a birational (rational) map. Subtracting off the divisorial base-locus (which does not affect the rational map) we may further assume the base locus is at most zero dimensional. Now $W=\sum b_{i} D_{i}$ is effective, nef and big, and supported on $D$. We show 
we may assume that in addition $b_{i}>0$ and $W \cdot D_{i}>0$ for each $i$. If $W \cdot D_{i}>0$, then we may assume $b_{i}>0$ (by adding $\epsilon D_{i}$ to $W$ if necessary). Now consider the set $S \subset\{1, \ldots, n\}$ of components $D_{i}$ of $D$ such that $W \cdot D_{i}=0$. By connectedness of $D$ we find $b_{i}>0$ for each $i \in S$. Thus $\operatorname{Supp}(W)=D$. By the Hodge index theorem the intersection matrix $\left(D_{i} \cdot D_{j}\right)_{i, j \in S}$ is negative definite. Hence there exists a linear combination $E=\sum_{i \in S} \alpha_{i} D_{i}$, with $\alpha_{i} \in \mathbb{Z}$ for each $i \in S$, such that $E \cdot D_{i}<0$ for each $i \in S$. Now replacing $W$ by $W-\epsilon E$, we obtain $W \cdot D_{i}>0$ for each $i=1, \ldots, n$.

Finally we show (1.3) implies (1.1). Since $U=Y \backslash D$ is the resolution of an affine variety $U^{\prime}$ with du Val singularities, we have $U^{\prime}=Y^{\prime} \backslash D^{\prime}$ where $Y^{\prime}$ is a normal projective surface and $D^{\prime}$ is a Weil divisor such that $D^{\prime}$ is the support of an ample divisor $A$. Let $\pi: \tilde{Y} \rightarrow Y^{\prime}$ be a resolution of singularities such that $\left.\pi\right|_{\pi^{-1}\left(U^{\prime}\right)}: \pi^{-1}\left(U^{\prime}\right) \rightarrow U^{\prime}$ is the resolution $U \rightarrow U^{\prime}$. Furthermore, we can assume the inclusion $U \subset Y$ extends to a birational morphism $f: \tilde{Y} \rightarrow Y$. Let $\tilde{D}$ be the inverse image of $D^{\prime}$ under $\pi$, so $\tilde{Y} \backslash \tilde{D}=$ $U$. The divisor $\pi^{*} A$ has support $\tilde{D}$. So we can write $\pi^{*} A=f^{*}\left(\sum a_{i} D_{i}\right)+\sum \mu_{j} E_{j}$ where the $E_{j}$ are the $f$-exceptional curves and $a_{i}, \mu_{j} \in \mathbb{Z}$. Then $\left(\sum a_{i} D_{i}\right)^{2} \geq\left(\pi^{*} A\right)^{2}=$ $A^{2}>0$.

Corollary 6.11. Let $(Y, D)$ be a positive Looijenga pair. Let $P=\mathrm{NE}(Y)$. The multiplication rule Theorem 2.34 applied with $\mathfrak{D}=\mathfrak{D}^{\text {can }}$ determines a finitely generated $T^{D}$-equivariant $R=\mathbb{k}[P]$-algebra structure on the free $R$-module

$$
A=\bigoplus_{q \in B(\mathbb{Z})} R \cdot \vartheta_{q} .
$$

Furthermore, $\operatorname{Spec} A \rightarrow \operatorname{Spec} R$ is a flat affine family of Gorenstein semi-log canonical (SLC) surfaces with central fibre $\mathbb{V}_{n}$, and smooth generic fibre. Any collection of $\vartheta_{q}$ whose restrictions generate $A / \mathfrak{m}=H^{0}\left(\mathbb{V}_{n}, \mathcal{O}_{\mathbb{V}_{n}}\right)$ generate $A$ as an $R$-algebra. In particular the $\vartheta_{v_{i}}$ generate for $n \geq 3$.

Proof. Everything but the singularity statement follows from Theorem 0.2 . The Gorenstein SLC locus in the base is open, and $T^{D}$-equivariant. Taking a big and nef divisor $H=\sum a_{i} D_{i}$ with $a_{i}>0$ and $H \cdot D_{i}>0$ for all $i$, we obtain a one-parameter subgroup of $T^{D}$ given by the map $\chi\left(T^{D}\right) \rightarrow \mathbb{Z}, e_{D_{i}} \mapsto a_{i}$. By definition of the weights, the weights of $z^{[C]}$ for $C \in \mathrm{NE}(Y)$ and $\vartheta_{p}$ for $p \in B(\mathbb{Z})$ are all non-negative. Thus the corresponding torus $T_{H} \cong \mathbb{G}_{m}$ gives a contracting action. In particular, since $\mathbb{V}_{n}$ is Gorenstein and SLC, all fibres are Gorenstein and SLC. Moreover, the map

$$
H^{0}\left(Z, \mathcal{O}_{Z}\right) \rightarrow H^{0}\left(\mathfrak{Z}_{\mathfrak{m}}, \mathcal{O}_{\mathfrak{Z} \mathfrak{m}}\right)
$$

is injective, where $Z$ is the singular locus of the family $X \rightarrow S$. But since $R \rightarrow$ $H^{0}\left(\mathcal{O}_{\mathfrak{Z}_{\mathfrak{m}}}, \mathcal{O}_{\mathfrak{Z}_{\mathfrak{m}}}\right)$ is not injective, as shown in the proof of Theorem 0.1 , we deduce that 
the map $R \rightarrow H^{0}\left(Z, \mathcal{O}_{Z}\right)$ is not injective. Letting $f$ be in the kernel of the map, the fibres over Spec $R \backslash V(f)$ are smooth.

In Part II we will prove that when $D$ is positive, our mirror family admits a canonical fibrewise $T^{D}$-equivariant compactification $\mathcal{X} \subset(\mathcal{Z}, \mathcal{D})$. The restriction $(\mathcal{Z}, \mathcal{D}) \rightarrow$ $T_{Y}:=\operatorname{Pic}(Y) \otimes \mathbb{G}_{m}$ comes with a trivialization $\mathcal{D} \stackrel{\sim}{\rightarrow} D_{*} \times T_{Y}$. We will show that $(\mathcal{Z}, \mathcal{D})$ is the universal family of Looijenga pairs $\left(Z, D_{Z}\right)$ deformation equivalent to $(Y, D)$ together with a choice of isomorphism $D_{Z} \stackrel{\sim}{\rightarrow} D_{*}$. Now for any positive pair $\left(Z, D_{Z}\right)$ together with a choice of isomorphism $\phi: D_{Z} \stackrel{\sim}{\rightarrow} D_{*}$, our construction equips the complement $U=Z \backslash D_{Z}$ with canonical theta functions $\vartheta_{q}, q \in B_{(Z, D)}(\mathbb{Z})$. We will give a characterisation in terms of the intrinsic geometry of $\left(Z, D_{Z}\right)$. Changing the choice of isomorphism $\phi$ changes $\vartheta_{q}$ by a character of $T^{D}=\operatorname{Aut}^{0}\left(D_{*}\right)$, the identity component of $\operatorname{Aut}\left(D_{*}\right)$. Here we illustrate with two examples:

Example 6.12. Consider first the case $(Y, D)$ a 5-cycle of $(-1)$-curves on the (unique) degree 5 del Pezzo surface, Example 3.7. In this case $T^{D}=T_{Y}=\operatorname{Pic}(Y) \otimes_{\mathbb{Z}} \mathbb{G}_{m}$, and thus by the $T^{D}$-equivariance, all fibres of the restriction $\mathcal{X} \rightarrow T_{Y}$ are isomorphic. We consider the fibre over the identity $e \in T_{Y}$, thus specializing the equations of Example 3.7 by setting all $z^{D_{i}}=1$. It's well known that these equations define an embedding of the original $U=Y \backslash D$ into $\mathbb{A}^{5}$ - if we take the closure in $\mathbb{P}^{5}$ (for the standard compactification $\mathbb{A}^{5} \subset \mathbb{P}^{5}$ ) one checks easily we obtain $Y$ with $D$ the hyperplane section at infinity.

Now it is easy to compute the zeroes and poles:

$$
\left(\vartheta_{v_{i}}\right)=E_{i}+D_{i}-D_{i+2}-D_{i-2}
$$

(indices mod 5). In particular $\left\{\vartheta_{v_{i}}=0\right\}=E_{i} \cap U \subset U$, which characterizes $\vartheta_{v_{i}}$ up to scaling.

Example 6.13. Now let $\left(Y, D=D_{1}+D_{2}+D_{3}\right)$ be (the deformation type of) a cubic surface together with a triangle of lines. Let $\mathcal{X} \subset \operatorname{Spec}(\mathbb{k}[\mathrm{NE}(Y)]) \times \mathbb{A}^{3}$ be the canonical embedding given by $\vartheta_{i}:=\vartheta_{v_{i}}, i=1,2,3$. In this case, as we shall see in Part II, the scattering diagram is particularly beautiful, with every ray $\mathfrak{d}$ of rational slope occuring, with precisely six curves on the cubic surface contributing to $f_{\mathfrak{d}}$. We will also show in Part II that the mirror is given by the equation

$$
\vartheta_{1} \vartheta_{2} \vartheta_{3}=\sum_{i} z^{D_{i}} \vartheta_{i}^{2}+\sum_{i}\left(\sum_{j} z^{E_{i j}}\right) z^{D_{i}} \vartheta_{i}+\sum_{\pi} z^{\pi^{*} H}-4 z^{D_{1}+D_{2}+D_{3}} .
$$

Here the $E_{i j}$ are the interior $(-1)$-curves meeting $D_{i}$, and the sum over $\pi$ is the sum over all possible toric models $\pi: Y \rightarrow \bar{Y}$ of $(Y, D)$ to a pair $(\bar{Y}, \bar{D})$ isomorphic to $\mathbb{P}^{2}$ with its toric boundary. (Such $\pi$ are permuted simply transitively by the Weyl 
group $W\left(D_{4}\right)$ by [L81], Prop. 4.5, p. 283.) The same family, in the same canonical coordinates, was discovered by Oblomkov Ob04. As we learned from Dolgachev, after a change of variables (in $\mathbb{A}^{3}$ ), and restricting to $T_{Y}$ (the locus over which the fibers have at worst Du Val singularities) this is identified with the universal family of affine cubic surfaces (the complement to a triangle of lines on projective cubic surface) constructed by Cayley in C1869. The universal family of cubic surfaces with triangle is obtained as the closure in $\mathbb{A}^{3} \subset \mathbb{P}^{3}$. In particular, as in the first example, our mirror family compactifies naturally to the universal family of Looijenga pairs deformation equivalent to the original $(Y, D)$. There is again a geometric characterisation of $\vartheta_{i}$ (up to scaling): The linear system $\left|-K_{Y}-D_{i}\right|=\left|D_{j}+D_{k}\right|$ (here $\{i, j, k\}=\{1,2,3\}$ ) is a basepoint free pencil. It defines a ruling $\pi: Y \rightarrow \mathbb{P}^{1}$ which restricts to a double cover $D_{i} \rightarrow \mathbb{P}^{1}$. Let $\{a, b\} \subset \mathbb{P}^{1}$ be the branch points of $\left.\pi\right|_{D_{i}}$. Let $p=\pi\left(D_{j}+D_{k}\right) \in \mathbb{P}^{1}$. There is a unique point $q \in \mathbb{P}^{1} \backslash\{a, b, p\}$ fixed by the unique involution of $\mathbb{P}^{1}$ interchanging $a$ and $b$ and fixing $p$. Let $Q=\pi^{*}(q) \in\left|D_{j}+D_{k}\right|$ be the corresponding divisor. The curve $Q \subset \mathbb{P}^{3}$ is a smooth conic. In Part II we will show

Proposition 6.14. $\left(\vartheta_{i}\right)=Q-D_{j}-D_{k}$.

\section{LOOIJENGA'S CONJECTURE}

In this section we apply the main construction of this paper to give a proof of Looijenga's conjecture on smoothability of cusp singularities, Theorem 0.5 . The simple conceptual idea is explained in the introduction. Here we give the rather involved details.

7.1. Duality of cusp singularities. We review the notion of dual cusp singularities. By definition, a cusp is a normal surface singularity for which the exceptional locus of the minimal resolution is a cycle of rational curves. The self-intersections of these exceptional curves determine the analytic type of the singularity, see [L73]. Cusps have a quotient construction due to Hirzebruch [Hi73] which we explain here. See also [L81], III, $\S 2$ for this point of view.

Let $M=\mathbb{Z}^{2}$, and let $T \in \mathrm{SL}(M)$ be a hyperbolic matrix, i.e., $T$ has a real eigenvalue $\lambda>1$. Then $T$ determines a pair of dual cusps as follows: Let $w_{1}, w_{2} \in M_{\mathbb{R}}$ be eigenvectors with eigenvalues $\lambda_{1}=1 / \lambda, \lambda_{2}=\lambda$, chosen so that $w_{1} \wedge w_{2}>0$ (in the standard counter-clockwise orientation of $\mathbb{R}^{2}$ ). Let $\bar{C}, \bar{C}^{\prime}$ be the strictly convex cones spanned by $w_{1}, w_{2}$ and $w_{2},-w_{1}$, and let $C, C^{\prime}$ be their interiors, either of which is preserved by $T$. Let $U_{C}, U_{C^{\prime}}$ be the corresponding tube domains, i.e.,

$$
U_{C}:=\left\{z \in M_{\mathbb{C}} \mid \operatorname{Im}(z) \in C\right\} / M \subset M_{\mathbb{C}} / M=M \otimes \mathbb{G}_{m} .
$$


$T$ acts freely and properly discontinuously on $U_{C}, U_{C^{\prime}}$. Write $Y_{C}, Y_{C^{\prime}}$ for the holomorphic hulls of $U_{C} / \Gamma, U_{C^{\prime}} / \Gamma$, where $\Gamma$ is the group generated by $T$. These each have one additional point, $p \in Y_{C}, p^{\prime} \in Y_{C^{\prime}}$, and $\left(Y_{C}, p\right),\left(Y_{C^{\prime}}, p^{\prime}\right)$ are normal surface germs of cusps.

Definition 7.1. $\left(Y_{C}, p\right)$ and $\left(Y_{C^{\prime}}, p^{\prime}\right)$ are dual cusp singularities.

All cusps (and their duals) arise this way.

Remark 7.2. If $M$ is identified with its dual by choosing an isomorphism $\bigwedge^{2} M \cong \mathbb{Z}$, the cone $\bar{C}^{\prime}$ is identified with the dual cone of $\bar{C}$. In this way $C^{\prime} / \Gamma$ and $C / \Gamma$ are dual integral affine manifolds, which suggests that the duality between the corresponding cusps is a form of mirror symmetry.

To resolve the cusp singularities of $Y_{C}$, say, one considers the convex hull $\Xi$ of integral points in $C$, and let $v_{i}, i \in \mathbb{Z}$, be the integral points in $\partial \Xi$, listed so that $v_{i-1}$ and $v_{i+1}$ are the integral points adjacent to $v_{i}$ on $\partial \Xi$. Let $\tilde{\Sigma}$ be the infinite fan with twodimensional cones generated by $v_{i-1}, v_{i}$ for $i \in \mathbb{Z}$. Note that $T$ acts on $\Xi$, and thus acts by translation $T\left(v_{i}\right)=v_{i+n}$ for some integer $n$, which we can take to be positive by reversing the ordering of the $v_{i}$ if necessary. Then $X_{\tilde{\Sigma}}$ is a toric variety with an infinite chain of $\mathbb{P}^{1}$ 's, and $T$ acts on $X_{\tilde{\Sigma}}$. There is a tubular neighbourhood $N$ of this infinite chain of $\mathbb{P}^{1}$ 's on which the group generated by $T$ acts properly discontinuously, see [AMRT75, p. 48. Then $N / \Gamma$ is a minimal resolution of singularities of a neighbourhood of the singularity of $Y_{C}$. Note the exceptional divisors $D_{i}, i$ taken modulo $n$, with $D_{i}$ corresponding to the ray of $\tilde{\Sigma}$ generated by $v_{i}$, satisfy (1.2). Thus if there is a Looijenga pair $\left(Y, D^{\prime}\right)$ with $D^{\prime}=D_{1}^{\prime}+\cdots+D_{n}^{\prime}$ and $\left(D_{i}^{\prime}\right)^{2}=D_{i}^{2}$ for each $i$, the corresponding affine manifold with singularities is precisely $B=|\tilde{\Sigma}| / \Gamma$ by Example 1.10, and $B_{0}=C / \Gamma$.

In fact, the dual cusp singularity can be described directly from the cone $C$ and the polyhedron $\Xi$ :

Lemma 7.3. Let $T, \bar{C}, \Xi$ and the $v_{i}$ be as above, giving a cusp singularity $p \in Y_{C}$. Let $Z$ be the toric variety (only locally of finite type) associated to $\Xi$. Let $E \subset Z$ be the toric boundary of $Z$, an infinite chain of smooth rational curves corresponding to the boundary of $\Xi$. Then there exists a tubular neighbourhood $E \subset N \subset Z$ such that the $\Gamma$ action on $\Xi$ induces a properly discontinuous $\Gamma$ action on $N$. Let $F \subset \tilde{X}$ denote the quotient of $E \subset N$ by $\Gamma$. So $F$ is a cycle of smooth rational curves. Then $F \subset \tilde{X}$ can be contracted to a singularity $p^{\prime} \in X$, which is a copy of the dual cusp $p^{\prime} \in Y_{C^{\prime}}$. Moreover, $\tilde{X}$ is obtained from the minimal resolution of $p^{\prime} \in X$ by contracting all the $(-2)$-curves. 
Proof. Let $\tilde{\Sigma}^{\prime}$ be the normal fan for the polytope $\Xi$ and $\bar{C}^{\prime \prime}$ the closure of its support. We observe that $\bar{C}^{\prime \prime}$ coincides with the dual of $\bar{C}$, together with the induced $\Gamma$-action. By Remark 7.2, it follows that $\tilde{X}$ is a partial resolution of a copy of the dual cusp.

The surface $\tilde{X}$ has Du Val singularities of type $A$. Indeed, $v_{i}$ is a vertex of $\Xi$ iff $m_{i}:=-D_{i}^{2}>2$. The corresponding point of $Z$ is smooth if $m_{i}=3$ and a singularity of type $A_{m_{i}-3}$ if $m_{i}>3$ (by direct calculation using $v_{i-1}+v_{i+1}=m_{i} v_{i}$ ). Also $K_{\tilde{X}}$ is relatively ample over $X$ by Lemma 7.4 below. Indeed, the vectors $u_{i}:=v_{i}-v_{i-1}$ are the primitive integral vectors in the direction of the edges of $\Xi$, and

$$
u_{i+1}-u_{i}=v_{i+1}+v_{i-1}-2 v_{i}=\left(m_{i}-2\right) v_{i},
$$

so the lines $v_{i}+\mathbb{R} \cdot\left(u_{i+1}-u_{i}\right)=\mathbb{R} \cdot v_{i}$ all meet at the origin. We deduce that $\tilde{X}$ is obtained from the minimal resolution of $X$ by contracting all (-2)-curves as claimed.

Lemma 7.4. Let $P \subset \mathbb{R}^{2}$ be a rational convex polygon and $X$ the associated toric surface. Fix an orientation of the boundary of $P$ and let $e_{0}, e_{1}, e_{2}$ denote oriented consecutive edges of the boundary of $P$. Let $C \subset X$ denote the component of the toric boundary associated to the bounded edge $e_{1} \subset P$. Let $v_{0}=e_{0} \cap e_{1}$ and $v_{1}=e_{1} \cap e_{2}$ be the vertices of $e_{1}$. Let $u_{0}, u_{1}, u_{2} \in \mathbb{Z}^{2}$ denote the primitive integral vectors in the direction of $e_{0}, e_{1}, e_{2}$. Then $K_{X} \cdot C>0$ if and only if the lines $v_{0}+\mathbb{R}\left(u_{1}-u_{0}\right)$ and $v_{1}+\mathbb{R}\left(u_{2}-u_{1}\right)$ meet on the opposite side of $e_{1}$ to $P$.

Proof. Write $M=\mathbb{Z}^{2} \subset \mathbb{R}^{2}$ for the lattice of characters of the torus of $X$. Choose an orientation $\bigwedge^{2} M \simeq \mathbb{Z}$ and use it to identify $M$ with its dual lattice $N$. Let $U \subset X$ denote the union of the two toric affine open subsets corresponding to the vertices $v_{0}$ and $v_{1}$ of $P$. Then $U$ is a toric open neighbourhood of $C \subset X$. Then, under this identification and up to a sign, the fan $\Sigma$ of $U$ in $N_{\mathbb{R}}$ consists of the two cones $\left\langle u_{0}, u_{1}\right\rangle_{\mathbb{R}_{\geq 0}},\left\langle u_{1}, u_{2}\right\rangle_{\mathbb{R}_{\geq 0}}$ and their faces. The condition on the lines $v_{0}+\mathbb{R}\left(u_{1}-u_{0}\right)$ and $v_{1}+\mathbb{R}\left(u_{2}-u_{1}\right)$ in the statement is equivalent to the condition that the primitive generator $u_{1}$ of the central ray of the fan $\Sigma$ lies on the same side of the affine line spanned by the primitive generators $u_{0}$ and $u_{2}$ of the two outer rays of $\Sigma$ as the origin $0 \in N$. Now by [R83], 4.3, this condition is equivalent to $K_{X} \cdot C>0$.

Because this quotient construction is analytic we will have to deal with convergence issues to show that our construction extends to this analytic situation.

7.2. Cusp family. In this subsection, we fix the following. Let $(Y, D)$ be a rational surface with anti-canonical cycle, now over the field $\mathbb{k}=\mathbb{C}$. We obtain $(B, \Sigma)$, with $\Sigma$ having one-dimensional cones $\rho_{i}$ and two-dimensional cones $\sigma_{i, i+1}$ as usual.

We assume that the intersection matrix $\left(D_{i} \cdot D_{j}\right)_{1 \leq i, j \leq n}$ is negative definite. Let $f: Y \rightarrow Y^{\prime}$ be the contraction (in the analytic category) of $D \subset Y$ to a cusp singularity 
$q \in Y^{\prime}$. We assume that $f$ is the minimal resolution of $Y^{\prime}$, that is, $D_{i}^{2} \leq-2$ for all $i$. We further assume that $n \geq 3$ to avoid additional technical issues of the flavour dealt with in $\$ 6.2$. The case of Looijenga's conjecture with $n \leq 2$ is in fact trivial, see the proof of Theorem 7.13 .

Let $L$ be a nef divisor on $Y$ such that

$$
\mathrm{NE}(Y)_{\mathbb{R}_{\geq 0}} \cap L^{\perp}=\left\langle D_{1}, \ldots, D_{n}\right\rangle_{\mathbb{R}_{\geq 0}}
$$

Here the subscript $\mathbb{R}_{\geq 0}$ denotes the real cone in $A_{1}(Y, \mathbb{R})$ generated by the given elements or set. (Indeed, if $Y^{\prime}$ is projective we can take $L=h^{*} A$ for $A$ an ample divisor on $Y^{\prime}$. In general, let $A$ be an ample divisor on $Y$. There exist unique $a_{i} \in \mathbb{Q}$ such that $L:=A+\sum a_{i} D_{i}$ is orthogonal to $D_{j}$ for each $j$. By negative definiteness of $\left\langle D_{1}, \ldots, D_{n}\right\rangle$, we have $a_{i}>0$ for each $i$. It follows that $L$ is nef.)

Let $\sigma_{P} \subset A_{1}(Y, \mathbb{R})$ be a rational polyhedral cone containing $\mathrm{NE}(Y), P=\sigma_{P} \cap$ $A_{1}(Y, \mathbb{Z})$ the associated toric monoid. We assume that $\sigma_{P}$ is strictly convex and $\sigma_{P} \cap L^{\perp}$ is a face of $\sigma_{P}$. Let $\mathfrak{m}=P \backslash\{0\}$ and $J=P \backslash P \cap L^{\perp} \subset P$, the radical monomial ideal associated to the face $\sigma_{P} \cap L^{\perp}$ of $\sigma_{P}$. We will write $S=\operatorname{Spec} \mathbb{k}[P]$, and for any monomial ideal $I$, we write $S_{I}=\operatorname{Spec} \mathbb{k}[P] / I$.

We take the multivalued piecewise linear function $\varphi$ as usual to have bending parameter $\kappa_{\rho, \varphi}=\left[D_{\rho}\right] \in P$. We wish to build a deformation of the $n$-vertex over $S_{I}$ with $\sqrt{I}=J$. However, this is already a problem over $S_{J}$ because none of the $\kappa_{\rho, \varphi}$ lie in $J$. Thus we can’t apply the results of $₫ 2$ directly as the standard open sets $U_{\rho, J}$ will not glue compatibly because of issues involving triple intersections. To deal with this, we need to shrink these open sets. This procedure is carried out as follows.

Theorem 7.5. Fix $R>1$. There exists an analytic open neighbourhood $S_{J}^{\prime}$ of $0 \in S_{J}$ and an analytic flat family $f_{J}: X_{J} \rightarrow S_{J}^{\prime}$ together with a section $s: S_{J}^{\prime} \rightarrow X_{J}$ satisfying the following properties:

(1) The general fibre $X_{J, t}$ of $f_{J}$ is a Stein analytic surface with a unique singularity $s(t) \in X_{J, t}$ isomorphic to the dual cusp to $q \in Y^{\prime}$.

(2) For each ray $\rho_{i} \in \Sigma$ there is an open analytic subset $V_{\rho_{i}, J} \subset X_{J}$ and open analytic embeddings

$V_{\rho_{i}, J} \subset\left\{\left(X_{i-1}, X_{i}, X_{i+1}\right) \in U_{\rho_{i}, J}|| X_{i-1}|<R| X_{i}|,| X_{i+1}|<R| X_{i} \mid\right\} \subset U_{\rho_{i}, J}$

where

$$
U_{\rho_{i}, J}:=V\left(X_{i-1} X_{i+1}-z^{\left[D_{\rho_{i}}\right]} X_{i}^{-D_{\rho_{i}}^{2}}\right) \subset \mathbb{A}_{X_{i-1}, X_{i+1}}^{2} \times\left(\mathbb{G}_{m}\right)_{X_{i}} \times S_{J}
$$

such that

(a) $X_{J}^{o}:=X_{J} \backslash s\left(S_{J}^{\prime}\right)=\bigcup_{\rho \in \Sigma} V_{\rho, J}$. 
(b) $V_{\rho, J} \cap V_{\rho^{\prime}, J}=\emptyset$ unless $\rho=\rho^{\prime}$ or $\rho$ and $\rho^{\prime}$ are the edges of a maximal cone $\sigma \in \Sigma$.

(3) The restriction of $X_{J} / S_{J}^{\prime}$ to $S_{J+\mathfrak{m}^{N+1}}$ is identified with an analytic neighbourhood of the vertex in the restriction of the family $X_{\mathfrak{m}^{N+1}} / S_{\mathfrak{m}^{N+1}}$ given by Theorem 2.28, (1) with $\mathfrak{D}=\mathfrak{D}^{\text {can }}$, for each $N \geq 0$.

Proof. (1) We use the notation of Example 1.10, so that the pair $(Y, D)$ determines an infinite fan $\tilde{\Sigma}$ in $M_{\mathbb{R}}$ with the primitive generators of the rays being the $v_{i}$ for $i \in \mathbb{Z}$. We also have $T \in \operatorname{SL}(M)$ acting on the fan $\tilde{\Sigma}$. We have $B=|\tilde{\Sigma}| / \Gamma$, where $\Gamma$ is the group generated by $T$.

Now as in $\S 7.1$, let $\Xi \subset M_{\mathbb{R}}$ be the convex hull of the points $v_{i} \in M$. Thus $\Xi$ is an infinite convex polytope. Let $\tilde{\Sigma}^{\prime}$ be the subdivision of $\Xi$ induced by $\tilde{\Sigma}$. In what follows, we will build a Mumford degeneration $Z / S_{J}$ with special fibre $Z_{0}$ the stable toric variety associated to $\tilde{\Sigma}^{\prime}$. In other words, $Z_{0}$ will be the union of the toric surfaces associated to the maximal polytopes in $\tilde{\Sigma}^{\prime}$.

$S_{J}$ is the affine toric variety associated to the face $\sigma_{\mathrm{bdy}}:=\sigma_{P} \cap L^{\perp}$ of $\sigma_{P}$, and $P_{\text {bdy }}:=\sigma_{\text {bdy }} \cap A_{1}(Y, \mathbb{Z})$ contains the classes of the components of the boundary of $Y$. We define a piecewise linear convex function $\tilde{\varphi}:\left|\tilde{\Sigma}^{\prime}\right| \rightarrow P_{\text {bdy }}^{\mathrm{gp}} \otimes_{\mathbb{Z}} \mathbb{R}$ by restriction of a piecewise linear convex function $\tilde{\varphi}$ on $|\tilde{\Sigma}|$. This function is the single-valued representative for $\varphi$ on the universal cover of $B_{0}$, and as such, is defined up to an integral linear function by specifying its bending parameter

$$
\kappa_{\tilde{\varphi}, \rho_{i}}=\left[D_{i \bmod n}\right] \in P_{\mathrm{bdy}}
$$

if $\rho_{i}=\mathbb{R}_{\geq 0} v_{i}$.

Then $\tilde{\varphi}$ determines a Mumford degeneration: this is a slight generalization of $\$ 1.3$. One defines

$$
\tilde{\Xi}:=\left\{(m, r) \mid m \in \Xi, r \in \tilde{\varphi}(m)+\sigma_{\text {bdy }}\right\} \subset M_{\mathbb{R}} \oplus\left(P_{\text {bdy }}^{\text {gp }} \otimes_{\mathbb{Z}} \mathbb{R}\right) .
$$

Let $C(\tilde{\Xi})$ be the closure of

$$
\{(s m, s r, s) \mid(m, r) \in \tilde{\Xi}, s \geq 0\} \subset M_{\mathbb{R}} \oplus\left(P_{\text {bdy }}^{\text {gp }} \otimes_{\mathbb{Z}} \mathbb{R}\right) \oplus \mathbb{R} .
$$

Then $\mathbb{C}\left[C(\tilde{\Xi}) \cap\left(M \oplus P_{\text {bdy }}^{\text {gp }} \oplus \mathbb{Z}\right)\right]$ has a natural grading given by the last coordinate, and the degree zero part of this ring is easily seen to contain $\mathbb{C}\left[P_{\text {bdy }}\right]$. Thus we obtain the Mumford family determined by $\tilde{\varphi}$ as

$$
Z:=\operatorname{Proj} \mathbb{C}\left[C(\tilde{\Xi}) \cap\left(M \oplus P_{\text {bdy }}^{\mathrm{gp}} \oplus \mathbb{Z}\right)\right] \rightarrow \operatorname{Spec} \mathbb{C}\left[P_{\text {bdy }}\right]=S_{J} .
$$

One sees easily that the fibre over $0 \in S_{J}$ of $Z \rightarrow S_{J}$ has infinitely many components indexed by the 2-cells of the subdivision $\tilde{\Sigma}^{\prime}$ of $\Xi$, each of which is a copy of the blowup of $\mathbb{A}^{2}$ at the origin. The general fibre is a toric surface (only locally of finite type) 
containing an infinite chain of smooth rational curves, which specializes to the union of the exceptional curves of the blowups in $Z_{0}$. By construction $\Gamma$ acts on $Z$ over $S_{J}$ (because $\tilde{\varphi}$ is $\Gamma$-invariant modulo integral affine functions). Let $E \subset Z / S_{J}$ be the family of curves described above (the relative toric boundary). The group $\Gamma$ acts properly discontinuously on a tubular neighbourhood $N$ of $E \subset Z$ (cf. [AMRT75, p. 48). Let $p:(F \subset \tilde{X}) \rightarrow S_{J}$ denote the quotient of $(E \subset N) \rightarrow S_{J}$ by $\Gamma$.

The divisor $F \subset \tilde{X}$ is Cartier and the dual of its normal bundle is relatively ample over a neighbourhood of $0 \in S_{J}$. Indeed, the special fibre $\tilde{X}_{0}$ is a union of $n$ irreducible components each isomorphic to a tubular neighbourhood of the exceptional locus in the blowup of $\mathbb{A}^{2}$, and $F_{0} \subset \tilde{X}_{0}$ is the cycle of $n$ smooth rational curves formed by the exceptional curves of the blowups. Hence the normal bundle of $F_{0}$ in $\tilde{X}_{0}$ has degree -1 on each component of $F_{0}$. Moreover, we have $R^{1} p_{*}\left(\mathcal{N}_{F / \tilde{X}}^{\vee}\right)^{\otimes k}=0$ for each $k>0$. Indeed, by cohomology and base change it suffices to show that $H^{1}\left(\left(\mathcal{N}_{F_{0} / \tilde{X}_{0}}^{\vee}\right)^{\otimes k}\right)=0$, and this follows from Serre duality. Now by a relative version of Grauert's contractibility criterion [F75], Thm. 2, taking global sections of the structure sheaf defines a contraction $p: \tilde{X} \rightarrow X_{J} / S_{J}$ to a family of Stein analytic spaces with exceptional locus $F$. The general fibre $X_{J, t}$ of $X_{J} / S_{J}$ is the dual cusp by Lemma 7.3. The section $s: S_{J} \rightarrow X_{J}$ takes $x \in S_{J}$ to the cusp of $X_{J, x}$.

We now show that $X_{J} / S_{J}$ is flat and the special fibre is the neighbourhood of the $n$-vertex obtained by contracting $F_{0} \subset \tilde{X}_{0}$. The key point is that $R^{1} p_{*} \mathcal{O}_{\tilde{X}}$ is a locally free $\mathcal{O}_{S_{J}}$-module, cf. [W76], Theorem 1.4(b). Indeed, we have

$$
R^{1} p_{*} \mathcal{O}_{\tilde{X}}(-F)=0
$$

by cohomology and base change, the theorem on formal functions, and the vanishing $H^{1}\left(\left(\mathcal{N}_{F_{0} / \tilde{X}_{0}}^{\vee}\right)^{\otimes k}\right)=0$ for $k>0$ used above. So, pushing forward the exact sequence

$$
0 \rightarrow \mathcal{O}_{\tilde{X}}(-F) \rightarrow \mathcal{O}_{\tilde{X}} \rightarrow \mathcal{O}_{F} \rightarrow 0
$$

we obtain

$$
R^{1} p_{*} \mathcal{O}_{\tilde{X}}=R^{1} p_{*} \mathcal{O}_{F} \simeq \mathcal{O}_{S_{J}}
$$

Recall that $S_{J}$ is a toric variety, so in particular Cohen-Macaulay. Let $t_{1}, \ldots, t_{r}$ be a regular sequence at $0 \in S_{J}$ of length $\operatorname{dim} S_{J}$ and write

$$
S_{J}^{i}=V\left(t_{1}, \cdots, t_{i}\right) \subset S_{J}
$$

$\tilde{X}^{i}=\left.\tilde{X}\right|_{S_{J}^{i}}$, and let $X_{J}^{i} / S_{J}^{i}$ be the family over $S_{J}^{i}$ defined by $\mathcal{O}_{X_{J}^{i}}=p_{*} \mathcal{O}_{\tilde{X}^{i}}$. Arguing as above we find that $R^{1} p_{*} \mathcal{O}_{\tilde{X}^{i}} \simeq \mathcal{O}_{S_{J}^{i}}$. Pushing forward the exact sequence

$$
0 \rightarrow \mathcal{O}_{\tilde{X}^{i}} \stackrel{t_{i+1}}{\longrightarrow} \mathcal{O}_{\tilde{X}^{i}} \longrightarrow \mathcal{O}_{\tilde{X}^{i+1}} \longrightarrow 0
$$


we deduce that the natural map

$$
\mathcal{O}_{X_{J}^{i}} / t_{i+1} \mathcal{O}_{X_{J}^{i}} \rightarrow \mathcal{O}_{X_{J}^{i+1}}
$$

is an isomorphism. Hence by the local criterion of flatness [Ma89], Ex. 22.3, p. 178, it suffices to show that $X_{J}^{r} / S_{J}^{r}$ is flat with special fibre the $n$-vertex. But $S_{J}^{r}$ is the spectrum of an Artinian $\mathbb{C}$-algebra, so this follows from [W76], Theorem 1.4(b).

(2) Write $Z^{o}=Z \backslash E$, and $m_{i}=-D_{i \bmod n}^{2}, a_{i}=z^{\left[D_{i \bmod n}\right]}$ for $i \in \mathbb{Z}$. We have an open covering

$$
Z^{o}=\bigcup_{i \in \mathbb{Z}} U_{i, J}
$$

where

$$
U_{i, J}=V\left(x_{i-1} x_{i+1}-a_{i} x_{i}^{m_{i}}\right) \subset \mathbb{A}_{x_{i-1}, x_{i+1}}^{2} \times\left(\mathbb{G}_{m}\right)_{x_{i}} \times S_{J} .
$$

Similarly, we have an open covering

$$
Z=\bigcup_{i \in \mathbb{Z}} \bar{U}_{i, J}
$$

where

$$
\begin{aligned}
\bar{U}_{i, J} & =V\left(x_{i-1}^{\prime} x_{i+1}^{\prime}-a_{i} x_{i}^{m_{i}-2}\right) \subset \mathbb{A}_{x_{i-1}^{\prime}, x_{i}, x_{i+1}^{\prime}}^{3} \times S_{J}, \\
E \cap \bar{U}_{i, J} & =V\left(x_{i}\right) \subset \bar{U}_{i, J},
\end{aligned}
$$

and $\bar{U}_{i, J} \backslash E=U_{i, J}$ via $x_{i-1}=x_{i} x_{i-1}^{\prime}, x_{i+1}=x_{i} x_{i+1}^{\prime}$. (Note that $m_{i}=-D_{i}^{2} \geq 2$ by assumption.)

Recall that the infinite cyclic group $\Gamma$ acts on $Z / S_{J}$, there is a $\Gamma$-invariant tubular neighbourhood $N \subset Z$ of $E \subset Z$ on which the action is properly discontinuous, and $F \subset \tilde{X}$ is obtained as the quotient of $E \subset N$ by $\Gamma$. In terms of the open covering above the action is given by $U_{i, J} \rightarrow U_{i+n, J}, x_{j} \mapsto x_{j+n}$. Note that the map $U_{i, J} \cap N \rightarrow \tilde{X}$ is not an open embedding (because, for example, $U_{i, J}$ contains the general fibre of $Z^{\circ} / S_{J}$ ). Fix $R>1$. We define $W_{i} \subset U_{i, J} \cap N$ by

$$
W_{i}=\left\{\left(x_{i-1}, x_{i}, x_{i+1}\right) \in U_{i, J} \cap N|| x_{i-1}|<R| x_{i}|,| x_{i+1}|<R| x_{i} \mid\right\}
$$

and similarly define $\bar{W}_{i} \subset \bar{U}_{i, J} \cap N$ by

$$
\bar{W}_{i}=\left\{\left(x_{i-1}^{\prime}, x_{i}, x_{i+1}^{\prime}\right) \in \bar{U}_{i, J} \cap N|| x_{i-1}^{\prime}|<R,| x_{i+1}^{\prime} \mid<R\right\} .
$$

Then $\bar{W}_{i} \backslash E=W_{i}$.

The $\bar{W}_{i}$ cover the special fibre $E_{0}$ of $E / S_{J}$ (using $R>1$ ). The open set $\bigcup \bar{W}_{i} \subset N$ is $\Gamma$-invariant, the quotient $(N, E) \rightarrow(\tilde{X}, F)$ by $\Gamma$ is a covering map, and $p: \tilde{X} \rightarrow X_{J}$ is proper with exceptional locus $F$. Hence we may assume (passing to an analytic neighbourhood $S_{J}^{\prime}$ of $0 \in S_{J}$ and $s(0) \in X_{J}$ ) that $N=\bigcup \bar{W}_{i}$. 
By Lemma 7.6 below there exists $\delta>0$ such that

$$
\bar{W}_{i} \cap\left\{\left(x_{i-1}, x_{i}, x_{i+1}\right)|| x_{i} \mid<\delta\right\} \subset\left\{\left|x_{j}\right|<1 \quad \forall j\right\}
$$

for each $i$. We replace $\bar{W}_{i}$ by $\bar{W}_{i} \cap\left\{\left|x_{i}\right|<\delta\right\}$, and modify $W_{i}$ similarly. Then as above we may assume that $N=\bigcup \bar{W}_{i}$, and $N \subset\left\{\left|x_{i}\right|<1 \quad \forall i\right\}$. We claim that $W_{i} \cap W_{j}=\emptyset$ for all $j>i+1$. It suffices to work on the general fibre of $Z^{o} / S_{J}^{\prime}$, which is an algebraic torus. The coordinate functions $x_{i}$ are characters of this torus (up to a multiplicative constant). By construction we have $\left|x_{i}\right|<1$ for each $i$ on $N$. Hence, shrinking the base $S_{J}^{\prime}$, we may assume that $\left|a_{i} x_{i}^{m_{i}-2}\right|<1 / R^{2}$ for each $i$. The relation

$$
x_{i-1} x_{i+1}=a_{i} x_{i}^{m_{i}}
$$

gives the inequality

$$
\left|x_{i+1} / x_{i}\right|<\left(1 / R^{2}\right)\left|x_{i} / x_{i-1}\right| .
$$

Combining such inequalities we obtain

$$
\left|x_{j+1} / x_{j}\right|<\left(1 / R^{2}\right)^{j-i}\left|x_{i+1} / x_{i}\right| \quad \text { for } j>i .
$$

Now $\left|x_{i+1} / x_{i}\right|<R$ on $W_{i}$ and $\left|x_{j-1} / x_{j}\right|<R$ on $W_{j}$, so $\left|x_{j} / x_{j-1}\right|>\left(1 / R^{2}\right)\left|x_{i+1} / x_{i}\right|$ on $W_{i} \cap W_{j}$. For $j>i+1$ this contradicts the inequality (17.2), hence $W_{i} \cap W_{j}=\emptyset$ as claimed.

It follows that $W_{i}$ embeds in $X_{J}$, using the fact that we have assumed $n \geq 3$. Let $V_{i}$ denote its image, with indices now understood modulo $n$. Thus $X_{J}^{o}=\bigcup V_{i}$ and the inverse image of $V_{i}$ is the (disjoint) union of $W_{j}$ such that $j \equiv i \bmod n$. We have $V_{i} \cap V_{j}=\emptyset$ for $j \neq i-1, i, i+1$ by our claim above. So, writing $V_{\rho, J}:=V_{i}$ for $\rho$ the ray of $\Sigma$ corresponding to $D_{i}$, the condition $(2)(\mathrm{b})$ is satisfied.

(3) We have the open covering $X_{J}^{o}=\bigcup V_{i}$ and open embeddings $V_{i} \subset U_{i, J}$, and an open covering $X_{\mathfrak{m}^{N+1}}^{o}=\bigcup U_{i, \mathfrak{m}^{N+1}}$. The restrictions of $U_{i, J} / S_{J}$ and $U_{i, \mathfrak{m}^{N+1}} / S_{\mathfrak{m}^{N+1}}$ to $S_{J+\mathfrak{m}^{N+1}}$ are identified, and the gluing maps coincide. It follows that the restriction of $X_{J} / S_{J}$ is identified with a neighbourhood of the vertex in the restriction of $X_{\mathfrak{m}^{N+1}} / S_{\mathfrak{m}^{N+1}}$ using Lemma 2.10.

Lemma 7.6. We use the notation of the proof of Theorem 7.5(2). Let $x_{j}$ be the coordinate functions on $Z^{\circ}=\bigcup U_{i, J}$. There exists $\delta>0$ such that on each open set $U_{i, J}$ if $\left|x_{i}\right|<\delta,\left|x_{i-1}\right|<R\left|x_{i}\right|,\left|x_{i+1}\right|<R\left|x_{i}\right|$, and $\left|z^{p}\right|<1$ for all $p \in P_{\text {bdy }}$ then $\left|x_{j}\right|<1$ for all $j$.

Proof. The points $v_{i-1}, v_{i}$, and $v_{i+1}$ are consecutive integral points on the boundary of the infinite convex polytope $\Xi$ with asymptotic directions $w_{1}, w_{2}$. It follows that $w_{1}=\alpha_{i 1} v_{i}+\beta_{i 1}\left(v_{i-1}-v_{i}\right)$ and $w_{2}=\alpha_{i 2} v_{i}+\beta_{i 2}\left(v_{i+1}-v_{i}\right)$ for some $\alpha_{i 1}, \beta_{i 1}, \alpha_{i 2}, \beta_{i 2} \in \mathbb{R}_{>0}$. Note that the ratios $\beta_{i 1} / \alpha_{i 1}, \beta_{i 2} / \alpha_{i 2}$ only depend on $i$ modulo $n$ (because $w_{1}, w_{2}$ are 
eigenvectors of $T$ and $\left.T\left(v_{i}\right)=v_{i+n}\right)$. Let $\mu$ be the maximum of the ratios $\beta_{i 1} / \alpha_{i 1}, \beta_{i 2} / \alpha_{i 2}$ for $i=1, \ldots, n$. Let $\delta=R^{-\mu}$. If $j>i$ then $v_{j}=\alpha v_{i}+\beta\left(v_{i+1}-v_{i}\right)$ with $\beta / \alpha<\beta_{i 2} / \alpha_{i 2}$. The coordinate function $x_{j}$ can be written as $z^{p} x_{i}^{\alpha}\left(x_{i+1} / x_{i}\right)^{\beta}$ on $V_{i}$, where $p \in P_{\text {bdy }}$. Thus $\left|x_{j}\right|<\delta^{\alpha} R^{\beta}<1$ for $\left|x_{i}\right|<\delta$ and $\left|z^{p}\right|<1$. The same is true for $j<i$ by symmetry.

7.3. Thickening of the cusp family. We continue to work with the setup at the beginning of $\$ 7.2$, with $(Y, D), L, \sigma_{P}, P, \mathfrak{m}$ and $J$ as given there.

Theorem 7.7. Let $p_{J}: X_{J} \rightarrow S_{J}^{\prime}$ be the analytic family of Theorem 7.5. Possibly after replacing $S_{J}^{\prime}$ by a smaller neighbourhood of $0 \in S_{J}$ and $X_{J}$ by a smaller neighbourhood of $s\left(S_{J}^{\prime}\right) \subset X_{J}$, independent of the choice of $I$ below, the following holds. Let $I \subset P$ be a monomial ideal such that $\sqrt{I}=J$ and let $S_{I}^{\prime} \subset S_{I}$ denote the induced thickening of $S_{J}^{\prime} \subset S_{J}$. There is an infinitesimal deformation $f_{I}: X_{I} \rightarrow S_{I}^{\prime}$ of $f_{J}: X_{J} \rightarrow S_{J}^{\prime}$ such that for each $N>0$ the restriction to $\operatorname{Spec} \mathbb{k}[P] /\left(I+\mathfrak{m}^{N+1}\right)$ is identified with an analytic neighbourhood of the vertex in the restriction of the family $X_{\mathfrak{m}^{N+1}} / S_{\mathfrak{m}^{N+1}}$ given by Theorem 2.28, (1) applied with $\mathfrak{D}=\mathfrak{D}^{\mathrm{can}}$.

Proof. As usual, $\mathfrak{D}^{\text {can }}$ is the canonical scattering diagram on $B$ associated to the pair $(Y, D)$. Note that the hypotheses (I) and (II) of Theorem 3.8 are satisfied for the ideal $J$ (but not (III)). This is because we can take $L=A+\sum a_{i} D_{i}$ with $A$ ample on $Y$ and $a_{i}>0$, and any $\mathbb{A}^{1}$-class $\beta$ intersects the $D_{i}$ non-negatively. Then for any $k$ there are only a finite number of $\mathbb{A}^{1}$-classes $\beta$ such that $L \cdot \beta<k$. In addition, there are no $\mathbb{A}^{1}$-classes $\beta$ with $\beta \cdot L=0$.

Let $\mathfrak{D}$ be the scattering diagram obtained by reducing $\mathfrak{D}^{\text {can }}$ modulo $I$ as follows. For each ray $\mathfrak{d}$ of $\mathfrak{D}^{\text {can }}$, we truncate the attached function $f_{\mathfrak{d}}$ by removing monomial terms lying in $I \cdot \mathbb{k}\left[P_{\varphi_{\tau_{\mathfrak{D}}}}\right]$, and we discard the ray if the truncated function equals 1 . Because of (II), $\mathfrak{D}$ has only finitely many rays $\mathfrak{d}$ and the attached functions $f_{\mathfrak{o}}$ are finite sums of monomials. Because of (I), $\mathfrak{D}$ is empty if $I=J$.

We use the scattering diagram $\mathfrak{D}$ to define a complex analytic space $X_{I}^{o} / S_{I}^{\prime}$ as follows. Recall from (2.7) the description of the schemes $U_{\rho_{i}, I} / S_{I}$ for $\rho_{i} \in \Sigma$ :

$$
U_{\rho_{i}, I}=V\left(X_{i-1} X_{i+1}-z^{\left[D_{\rho}\right]} X_{i}^{-D_{\rho}^{2}} f_{\rho}\right) \subset \mathbb{A}_{X_{i-1}, X_{i+1}}^{2} \times\left(\mathbb{G}_{m}\right)_{X_{i}} \times S_{I}
$$

We have open subsets $U_{\rho_{i}, \sigma_{i-1, i}, I}, U_{\rho_{i}, \sigma_{i, i+1}, I} \subset U_{\rho_{i}, I}$ defined by $X_{i-1} \neq 0$ and $X_{i+1} \neq 0$ respectively. We have canonical identifications

$$
U_{\rho_{i}, \sigma_{i, i+1}, I}=U_{\sigma_{i, i+1}, \sigma_{i, i+1}, I}=U_{\rho_{i+1}, \sigma_{i, i+1}, I}
$$

where

$$
U_{\sigma_{i, i+1}, \sigma_{i, i+1}}=\left(\mathbb{G}_{m}\right)_{X_{i}, X_{i+1}}^{2} \times S_{I}
$$


Recall that we have an open covering $X_{J}^{o}=\bigcup_{\rho \in \Sigma} V_{\rho, J}$ and open analytic embeddings $V_{\rho, J} \subset U_{\rho, J}$. Write

$$
V_{\sigma_{i, i+1}, \sigma_{i, i+1}, J}:=\left(V_{\rho_{i}, J} \cap U_{\rho_{i}, \sigma_{i, i+1}, J}\right) \cap\left(V_{\rho_{i+1}, J} \cap U_{\rho_{i+1}, \sigma_{i, i+1}, J}\right) \subset U_{\sigma_{i, i+1}, \sigma_{i, i+1}, J}
$$

where we use the identification (7.3). Let $V_{\rho_{i}, \sigma_{i, i+1}, J} \subset V_{\rho_{i}, J}, V_{\rho_{i+1}, \sigma_{i, i+1}, J} \subset V_{\rho_{i+1}, J}$ denote the open subsets corresponding to $V_{\sigma_{i, i+1}, \sigma_{i, i+1}, J}$ under (7.3). Let $V_{\rho_{i}, I}, V_{\rho_{i}, \sigma_{i, i+1}, I}$, etc., be the infinitesimal thickenings of these open sets determined by the thickenings $U_{\rho_{i}, I}$ of $U_{\rho_{i}, J}$. Let $\theta_{\gamma, \mathfrak{D}}: U_{\rho_{i+1}, \sigma_{i, i+1}, I} \rightarrow U_{\rho_{i}, \sigma_{i, i+1}, I}$ be the gluing isomorphism defined as in $\$ 2.2$. Note that as the canonical scattering diagram $\mathfrak{D}^{\text {can }}$ is trivial modulo $J, \theta_{\gamma, \mathfrak{D}}$ restricts to the identification (17.3) modulo $J$, and thus restricts to an isomorphism

$$
V_{\rho_{i+1}, \sigma_{i, i+1}, I} \rightarrow V_{\rho_{i}, \sigma_{i, i+1}, I}
$$

Gluing the $V_{\rho, I}$ via these isomorphisms we obtain an infinitesimal deformation $X_{I}^{o} / S_{I}^{\prime}$ of $X_{J}^{o} / S_{J}^{\prime}$. Note that there are no triple overlaps of the $V_{\rho, J}$ by Theorem $7.5(2)(\mathrm{b})$, hence no compatibility condition for the gluing automorphisms. It is clear from the construction that the families $X_{I}^{o} / S_{I}^{\prime}$ and $X_{\mathfrak{m}^{N+1}} / S_{\mathfrak{m}^{N+1}}$ are compatible.

We define sections $\vartheta_{q} \in \Gamma\left(X_{I}^{o}, \mathcal{O}_{X_{I}^{o}}\right)$ for $q \in B(\mathbb{Z})$, compatible with the sections of Theorem 2.28, (2). We proceed as in the algebraic case: we first define a local section $\operatorname{Lift}_{Q}(q)$ for each choice of basepoint $Q \in B_{0} \backslash \operatorname{Supp}(\mathfrak{D})$ on a corresponding open patch of $X_{I}^{o}$ using the broken lines construction. The new difficulty here is that the functions $\operatorname{Lift}_{Q}(q)$ are not algebraic, even over the unthickened locus $S_{J}^{\prime}$. Indeed, by definition $\operatorname{Lift}_{Q}(q)=\sum \operatorname{Mono}(\gamma)$ is a formal sum of monomials corresponding to broken lines $\gamma$ for $q$ with endpoint $Q$. Note that with our current choice of ideal $J$, this sum is always infinite, as is already evident in Example 2.18. So we must prove convergence. This is done in \$7.3.1, see Propositions 7.10 and 7.11.

Once this convergence is proved, we observe that these patch to give well-defined global sections. This follows from the consistency of $\mathfrak{D}^{\text {can }}$ and compatibility of $X_{I}^{o} / S_{I}^{\prime}$ with $X_{\mathfrak{m}^{N+1}}^{o} / S_{\mathfrak{m}^{N+1}}$ for $N \geq 0$.

We define an infinitesimal thickening $X_{I} / S_{I}^{\prime}$ of $X_{J} / S_{J}^{\prime}$ by $\mathcal{O}_{X_{I}}=i_{*} \mathcal{O}_{X_{I}^{o}}$ where $i: X_{J}^{o} \subset X_{J}$ is the inclusion. Then $X_{I} / S_{I}^{\prime}$ is flat by Lemma 2.29 and the existence of the lifts $\vartheta_{q}$.

7.3.1. Convergence of Lifts. Let $\bar{C} \subset M_{\mathbb{R}}$ be the closure of the support $|\tilde{\Sigma}|$ of the fan $\tilde{\Sigma}$, a closed convex cone. Let $w_{1}, w_{2}$ be generators of $\bar{C}$. Then $w_{1}, w_{2}$ are eigenvectors of $T$ with eigenvalues $\lambda^{-1}, \lambda$ for some $\lambda \in \mathbb{R}$. We may assume that $\lambda>1$. Let $\pi: \tilde{B}_{0} \rightarrow B_{0}$ denote the universal cover of $B_{0}$. So $\tilde{B}_{0}$ is identified with $C=\operatorname{Int}(\bar{C})$, with deck transformations given by the action of $\Gamma=\langle T\rangle$ on $C$. Let $\tilde{\mathcal{P}}, \tilde{\varphi}$, and $\tilde{B}_{0}(\mathbb{Z})$ denote the 
pullbacks of $\mathcal{P}, \varphi$, and $B_{0}(\mathbb{Z})$. Let $\tilde{\mathfrak{D}}$ denote the scattering diagram on $\tilde{B}_{0}$ induced by $\mathfrak{D}$. We fix a trivialization of $\tilde{\mathcal{P}}$ as the constant sheaf with fibre $P^{\mathrm{gp}} \oplus M$.

The behaviour of the broken lines $\gamma$ is best studied by passing to the universal cover $\tilde{B}_{0}$ of $B_{0}$. Let $Q \in B_{0}, q \in B_{0}(\mathbb{Z})$, and choose lifts $\tilde{Q} \in \tilde{B}_{0}, \tilde{q} \in \tilde{B}_{0}(\mathbb{Z})$. Then a broken line $\gamma$ on $B_{0}$ for $q$ with endpoint $Q$ lifts uniquely to a broken line $\tilde{\gamma}$ on $\tilde{B}_{0}$ for $T^{N}(\tilde{q})$ with endpoint $\tilde{Q}$, for some $N \in \mathbb{Z}$, and the attached monomials are identified via $\tilde{\mathcal{P}}=\pi^{*} \mathcal{P}$. Note that $T^{N}(\tilde{q})$ approaches $\mathbb{R}_{>0} \cdot w_{2}$ as $N \rightarrow \infty$ and $\mathbb{R}_{>0} \cdot w_{1}$ as $N \rightarrow-\infty$.

If $\gamma$ is a broken line for a point $\tilde{q} \in \tilde{B}_{0}(\mathbb{Z})$ with endpoint $\tilde{Q} \in \tilde{B}_{0}$ then $\gamma:(-\infty, 0] \rightarrow$ $\tilde{B}_{0}$ is a piecewise linear path in $\tilde{B}_{0}=C$ with initial direction $-\tilde{q}$, ending at $\tilde{Q}$, and crossing all the rays of $\tilde{\mathfrak{D}}$ between $\mathbb{R}_{\geq 0} \tilde{q}$ and $\mathbb{R}_{\geq 0} \tilde{Q}$ in order. Let $t_{1}, \ldots, t_{l} \in(-\infty, 0)$ denote the points where $\gamma$ is not affine linear. Each point $\gamma\left(t_{i}\right)$ lies on a ray $\mathfrak{d}_{i}$ of $\tilde{\mathfrak{D}}$ and the change $\gamma^{\prime}\left(t_{i}+\epsilon\right)-\gamma^{\prime}\left(t_{i}-\epsilon\right)$ in the direction of $\gamma$ as it crosses $\mathfrak{d}_{i}$ is an integral multiple of the primitive generator of $\mathfrak{d}_{i}$. Moreover this multiple is positive because each ray of the canonical scattering diagram is an outgoing ray in the terminology of Definition 2.13. So the path $\gamma$ is "convex when viewed from the origin".

It is convenient for the convergence calculation to decompose the monomials for broken lines as follows. Let $\gamma:(-\infty, 0] \rightarrow \tilde{B}_{0}$ be a broken line, $t \in(-\infty, 0]$ a point such that $\gamma$ is affine linear near $t$ and $\gamma(t)$ lies in the interior of a maximal cone $\sigma$ of $\tilde{\Sigma}$, and $c z^{q}$ the monomial attached to the domain of linearity of $\gamma$ containing $t$. Here $c \in \mathbb{k}$ and $q \in P_{\tilde{\varphi}_{\sigma}} \subset P^{\mathrm{gp}} \oplus M$. We write $c z^{q}=a z^{\tilde{\varphi}_{\sigma}(m)}$, where $m=r(q) \in M$ and $a=c z^{q-\tilde{\varphi}_{\sigma}(m)}$ is a monomial in $\mathbb{k}[P]$. We also use the same decomposition for the monomials occuring in the scattering functions $f_{\mathfrak{d}}$ for $\mathfrak{d} \in \tilde{\mathfrak{D}}$. Let $\mathfrak{d}$ be a ray in $\tilde{\mathfrak{D}}$ with primitive integral generator $m \in M$ and $\tau=\tau_{\mathfrak{d}}$ the smallest cone of $\tilde{\Sigma}$ containing $\mathfrak{d}$. Then $f_{\mathfrak{d}}-1$ is a sum of monomials $c z^{q}$ where $c \in \mathbb{k}$ and $q \in P_{\tilde{\varphi}_{\tau}}, 0 \neq-r(q) \in \mathfrak{d}$. We write $c z^{q}=a z^{\tilde{\varphi}_{\tau}(m)}$ where $m=r(q)$ and $a=c z^{q-\tilde{\varphi}_{\tau}(m)}$ is a monomial in $\mathbb{k}[P]$.

The scattering diagram $\mathfrak{D}$ on $B$ is finite (because we have reduced modulo $I$ ), and $\tilde{\mathfrak{D}}$ is its inverse image under $\pi: \tilde{B}_{0} \rightarrow B_{0}$. Thus $\tilde{\mathfrak{D}}$ has only finitely many $\Gamma$-orbits of rays. Moreover, the $\Gamma$-action on the scattering functions $f_{\mathfrak{o}}$ is induced by the given action on $M_{\mathbb{R}}$ and the trivial action on $\mathbb{k}[P]$ as follows: writing $f_{\mathfrak{d}}=1+\sum a_{m} z^{\tilde{\varphi}_{\tau}(m)}$ as above, $f_{T(\mathfrak{d})}=1+\sum a_{m} z^{\tilde{\varphi}_{T(\tau)}(T(m))}$.

Lemma 7.8. Let $\tilde{Q} \in \tilde{B}_{0} \backslash \operatorname{Supp}(\tilde{\mathfrak{D}})$ be a point contained in the interior of a maximal cone $\sigma \in \tilde{\Sigma}$. We consider broken lines $\gamma$ on $\tilde{B}_{0}$ for $T^{N}(\tilde{q})$, some $N \in \mathbb{Z}$, with endpoint $\tilde{Q}$, such that $\operatorname{Mono}(\gamma) \notin I \cdot \mathbb{k}\left[P_{\tilde{\varphi}_{\sigma}}\right]$. Let $k \geq 0$ be such that $(k+1) J \subset I$.

(1) The number of bends of $\gamma$ is at most $k$.

(2) The number of broken lines for $T^{N}(\tilde{q})$ with endpoint $\tilde{Q}$ is $O\left(|N|^{k}\right)$. 
(3) For $\gamma$ a broken line for $T^{N}(\tilde{q})$ with endpoint $\tilde{Q}$ write $\operatorname{Mono}(\gamma)=a_{\gamma} z^{\tilde{\varphi}_{\sigma}\left(m_{\gamma}\right)}$ where $a_{\gamma}$ is a monomial in $\mathbb{k}[P]$. Assume that $\left|z^{p}\right|<\epsilon<1$ for all $p \in P$. Then

$$
\left|a_{\gamma}\right|=O\left(\prod_{\substack{\rho \in \Sigma \\ \operatorname{dim} \rho=1}}\left|z^{\left[D_{\rho}\right]}\right|^{|N|}\right) .
$$

Proof. (1) At a bend $t_{i} \in(-\infty, 0]$ of $\gamma$ the attached monomial $c_{i} z^{q_{i}}$ is replaced by the monomial $c_{i+1} z^{q_{i+1}}=c z^{q} \cdot c_{i} z^{q_{i}}$ where $c z^{q}$ is a term in a positive power of the scattering function $f_{\mathfrak{d}}$ associated to the ray $\mathfrak{d}$ containing $\gamma\left(t_{i}\right)$. In particular $c z^{q} \in J \cdot \mathbb{k}\left[P_{\tilde{\varphi}_{\tau_{\mathfrak{d}}}}\right]$. Since $\operatorname{Mono}(\gamma) \notin I \cdot \mathbb{k}\left[P_{\tilde{\varphi}_{\sigma}}\right]$ and $(k+1) J \subset I$ it follows that there are at most $k$ bends.

(2) Such a broken line crosses $O(|N|)$ scattering rays. If $\gamma$ is a broken line for $T^{N}(\tilde{q})$ then the initial attached monomial is specified, equal to $z^{\tilde{\varphi}\left(T^{N}(\tilde{q})\right)}$. At a scattering ray $\mathfrak{d}$, let $u$ denote the primitive generator of $\mathfrak{d}, f=f_{\mathfrak{o}}$ the attached function, and let $c z^{q}$ be the monomial attached to the incoming segment of the broken line. Then the possible continuations of the broken line past $\mathfrak{d}$ correspond to the monomial terms in $f^{d}$, where $d=|r(q) \wedge u|$ is the index of the sublattice of $M$ generated by $r(q)$ and $u$. Note that since $f \equiv 1 \bmod J \cdot \mathbb{k}\left[P_{\tilde{\varphi}_{\tau_{0}}}\right]$ the number of monomial terms in $f^{d}$ not lying in $I \cdot \mathbb{k}\left[P_{\tilde{\varphi}_{\tau_{0}}}\right]$ is bounded independent of $d$. Further, since there are a finite number of $\Gamma$-orbits of scattering rays, and the $\Gamma$-action preserves monomials, there is a bound on the number of monomial terms independent of the ray $\mathfrak{d}$. Thus for a broken line $\gamma$ for $T^{N}(\tilde{q})$ there are $\left(\begin{array}{c}O(|N|) \\ k\end{array}\right)=O\left(|N|^{k}\right)$ choices of how it may bend by (1). So the total number of broken lines is $O\left(|N|^{k}\right)$.

(3) By symmetry we may assume that $N \geq 0$. Let $\mathfrak{d} \in \tilde{\mathfrak{D}}$ be a scattering ray, $f=f_{\mathfrak{o}}$ the attached function, and $\gamma$ a broken line that crosses $\mathfrak{d}$. Suppose first that $\mathfrak{d}$ is contained in the interior of a maximal cone $\sigma$ of $\tilde{\Sigma}$. Let $a z^{\tilde{\varphi}_{\sigma}(m)}$ be the monomial attached to the incoming segment of $\gamma$ near $\mathfrak{d}$. Let $u$ be the primitive generator of $\mathfrak{d}$. Then the outgoing monomial $a^{\prime} z^{\tilde{\varphi}_{\sigma}\left(m^{\prime}\right)}$ is obtained from the incoming monomial by multiplication by a monomial term in $f^{d}$, where $d=|m \wedge u|$. Write $f=1+f_{1}+\cdots+f_{r}$, a sum of monomials. Since $f \equiv 1 \bmod J \cdot \mathbb{k}\left[P_{\varphi_{\sigma}}\right]$ we have

$$
f^{d} \equiv \sum_{i_{1}+\cdots+i_{r} \leq k}\left(\begin{array}{c}
d \\
i_{1}, \ldots, i_{r}
\end{array}\right) f_{1}^{i_{1}} \cdots f_{r}^{i_{r}} \quad \bmod I \cdot \mathbb{k}\left[P_{\varphi_{\sigma}}\right] .
$$

The multinomial coefficient

$$
\left(\begin{array}{c}
d \\
i_{1}, \ldots, i_{r}
\end{array}\right):=\frac{d !}{i_{1} ! \cdots i_{r} !\left(d-i_{1}-\cdots-i_{r}\right) !}
$$

is bounded by $d^{k}$. The direction of the scattering ray $\mathfrak{d}$ is $u=T^{s}(\beta)$ where $0 \leq s \leq N$ and $\beta \in M$ is chosen from a finite set. 
The vector $m \in M$ is of the form

$$
m=T^{N}(\tilde{q})-\sum_{i=1}^{l} T^{s_{i}} \alpha_{i}
$$

where $l \leq k, 0 \leq s_{i} \leq N$ for each $i$, and the $\alpha_{i} \in M$ are chosen from a finite set. Indeed, as in the proof of (2), for the monomial terms $c z^{q}$ occurring in the powers $f^{d}$ of the function $f=f_{\mathfrak{d}}$ attached to a given scattering ray $\mathfrak{d}$, only finitely many exponents $q \in P_{\tilde{\varphi}_{\tau_{0}}}$ occur (working modulo $I \cdot \mathbb{k}\left[P_{\tilde{\varphi}_{\tau_{0}}}\right]$ ). So there are only finitely many possible changes of exponent $q$ for the attached monomial $c z^{q}$ of a broken line at a scattering ray modulo the action of $\Gamma$.

Now identify $M=\mathbb{Z}^{2}$ and let $\|\cdot\|$ denote the standard norm on $M_{\mathbb{R}}=\mathbb{R}^{2}$. Then

$$
d=|m \wedge u| \leq\|m\| \cdot\|u\|=O\left(\lambda^{2 N}\right) .
$$

So, the coefficient $a^{\prime} \in \mathbb{k}[P]$ of the outgoing monomial is given by $a^{\prime}=c \cdot z^{p} \cdot a$ where $c \in \mathbb{C}, p \in P$, and $|c|=O\left(\lambda^{2 k N}\right)$. Thus $\left|a^{\prime}\right|=O\left(\lambda^{2 k N}\right) \cdot|a|$ for $\left|z^{p}\right|<1$.

Next, let $\rho \in \tilde{\Sigma}$ be a ray and $\sigma_{+}, \sigma_{-}$the maximal cones containing $\rho$. Suppose $\gamma$ is a broken line that crosses $\rho$, travelling from $\sigma_{-}$to $\sigma_{+}$. Let $a_{-} z^{\tilde{\varphi}_{-}}(m)$ be the monomial attached to the incoming segment of $\gamma$ near $\rho$ and $a_{+} z^{\tilde{\varphi}_{\sigma_{+}}\left(m^{\prime}\right)}$ the monomial attached to the outgoing segment. By the definition of $\tilde{\varphi}$,

$$
z^{\tilde{\varphi}_{\sigma_{-}}(m)}=\left(z^{\left[D_{\rho}\right]}\right)^{-\left\langle n_{\rho}, m\right\rangle} z^{\tilde{\varphi}_{\sigma_{+}}(m)},
$$

where $n_{\rho} \in N$ is primitive, annihilates $\rho$, and is positive on $\sigma_{+}$. Write $d:=-\left\langle n_{\rho}, m\right\rangle$; note $d=|u \wedge m|>0$ where $u \in M$ is the primitive generator of $\rho$. If $\gamma$ does not bend at $\rho$ then $a_{+}=\left(z^{\left[D_{\rho}\right]}\right)^{d} \cdot a_{-}$. In general $a_{+}=\left(z^{\left[D_{\rho}\right]}\right)^{d} \cdot a_{-}^{\prime}$ where $a_{-}^{\prime} z^{\tilde{\varphi}_{\sigma_{-}}\left(m^{\prime}\right)}$ is obtained from $a_{-} z^{\tilde{\varphi} \sigma_{-}(m)}$ as above (by applying the scattering automorphism associated to $\rho$ and selecting a monomial term).

We need to show the exponent $d=|u \wedge m|>0$ of $z^{\left[D_{\rho}\right]}$ in the previous paragraph is large for some lift $\tilde{\rho}$ of any given ray $\rho \in \Sigma$. This will allow us to absorb the $O\left(\lambda^{2 N}\right)$ factors coming from bends of $\gamma$ and obtain the estimate (3). Let $\gamma$ be a broken line for $T^{N}(\tilde{q})$. Then

$$
m_{\gamma}=T^{N}(\tilde{q})-\sum_{i=1}^{l} T^{s_{i}} \alpha_{i}
$$

as above, where $0 \leq l \leq k, N \geq s_{1} \geq \cdots \geq s_{l} \geq 0$, and the $\alpha_{i}$ lie in a finite set. Write $s_{0}=N$ and $s_{l+1}=0$. Choose $j$ such that $s_{j}-s_{j+1} \geq N /(k+1)$. Now consider the exponent $d=|u \wedge m|$ for $m=T^{N}(\tilde{q})-\sum_{i=1}^{j} T^{s_{i}} \alpha_{i}$ given by the monomial attached to the segment of the broken line between bends $j$ and $j+1$. Let $\tilde{\rho} \in \tilde{\Sigma}$ be the lift of $\rho \in \Sigma$ between bends $j$ and $j+1$ which is closest to bend $j+1$ (such a lift exists if $N$ is sufficiently large). Let $u=T^{s_{j+1}} \beta$ be the primitive generator of $\tilde{\rho}$. Then 
$|u \wedge m|=\left|\beta \wedge T^{s_{j}-s_{j+1}} m^{\prime}\right|$ where $m^{\prime}=T^{-s_{j}}(m)$. Writing $m^{\prime}=\mu_{1}^{\prime} w_{1}+\mu_{2}^{\prime} w_{2}$, we see that $\left|\mu_{1}^{\prime}\right|$ is bounded since $w_{1}$ has eigenvalue $\lambda^{-1}<1$. Also, $\mu_{2}^{\prime}$ is bounded away from zero by Lemma 7.9. Now

$$
u \wedge m=\beta \wedge T^{s_{j}-s_{j+1}} m^{\prime}=\mu_{1}^{\prime}\left(\beta \wedge w_{1}\right) \lambda^{-\left(s_{j}-s_{j+1}\right)}+\mu_{2}^{\prime}\left(\beta \wedge w_{2}\right) \lambda^{s_{j}-s_{j+1}},
$$

where $s_{j}-s_{j+1}>N /(k+1)$, so

$$
|u \wedge m|>c \cdot \lambda^{N /(k+1)}
$$

for some constant $c>0$.

Combining our results now gives, when $\left|z^{\left[D_{\rho}\right]}\right|<1$ for all $p \in P_{\mathrm{bdy}}$, the estimate

$$
a_{\gamma}=O\left(\left(\lambda^{2 k N}\right)^{k} \cdot \prod_{\substack{\rho \in \Sigma \\ \operatorname{dim} \rho=1}}\left|z^{\left[D_{\rho}\right]}\right|^{c \cdot \lambda^{N /(k+1)}}\right) .
$$

where the first factor bounds the contribution associated to bends of $\gamma$ and the second factor bounds the contribution associated to rays $\rho$ of the fan crossed by $\gamma$, as described in the preceding two paragraphs. This implies the estimate (3) in the statement, using $\left|z^{\left[D_{\rho}\right]}\right|<1$. Indeed, the above expression is of the form

$$
\lambda^{a N} \cdot x^{c \cdot \lambda^{b N}}
$$

where $a, b, c>0$ and $\lambda>1$ are constants, and $x=\prod\left|z^{\left[D_{\rho}\right]}\right|$. This is bounded by $C x^{N}$ for $0 \leq x<\epsilon<1$, for some constant $C$ (depending on $\epsilon$ ). (To see this, we may assume $x \neq 0$, take logarithms, and establish an inequality

$$
a N \log \lambda+c \lambda^{b N} \log x \leq \log C+N \log x
$$

We have $-\log x>-\log \epsilon>0$. Rearranging (17.5), we require that, for some choice of $C$,

$$
a N \log \lambda \leq \log C+(-\log x)\left(c \lambda^{b N}-N\right)
$$

for all $N$. This holds because

$$
a N \log \lambda \leq(-\log x)\left(c \lambda^{b N}-N\right)
$$

for $N$ sufficiently large.)

Lemma 7.9. Let $A \subset \mathbb{R}$ be a finite set and $\lambda \in \mathbb{R}, \lambda>1$. For $k \in \mathbb{N}$ let $S_{k} \subset \mathbb{R}$ be the set of real numbers $s$ of the form $s=\sum_{i=1}^{l} c_{i} \lambda^{n_{i}}$ where $l \leq k$ and $c_{i} \in A, n_{i} \in \mathbb{Z}_{\geq 0}$ for each $i$. Then $S_{k}$ is discrete for each $k$.

Proof. Proof by induction on $k$. We have $S_{0}=\{0\}$. Suppose $S_{k}$ is discrete. We have $S_{k+1}=\bigcup_{n \geq 0} \lambda^{n}\left(S_{k}+A\right)$. Since $\lambda>1$ we deduce that $S_{k+1}$ discrete.

For Propositions 7.10 and 7.11 below, the assertions hold after possibly replacing $X_{J}$ by a smaller neighbourhood of $s(0) \in X_{J}$ (independent of $I, Q$ and $q$ ). 
Proposition 7.10. Let $Q \in B_{0} \backslash \operatorname{Supp}(\mathfrak{D})$ be a point contained in the interior of a maximal cone $\sigma$ of $\Sigma$. Each term of the formal sum $\operatorname{Lift}_{Q}(q)=\sum \operatorname{Mono}(\gamma)$ is an analytic function on $V_{\sigma, \sigma, I}$ and the sum defines an analytic function on $V_{\sigma, \sigma, I}$.

Proof. Recall that $V_{\sigma, \sigma, I}$ is an infinitesimal thickening of the reduced complex analytic space $V_{\sigma, \sigma, J}$. Write

$$
V_{\sigma, \sigma}:=\left\{\left(X_{1}, X_{2}\right)|| X_{1}|<R| X_{2}|,| X_{2}|<R| X_{1} \mid\right\} \subset\left(\mathbb{G}_{m}\right)_{X_{1}, X_{2}}^{2} \times S .
$$

Then $V_{\sigma, \sigma}$ is a reduced complex analytic space containing $V_{\sigma, \sigma, I}$ as a locally closed subspace. We show that the $\operatorname{sum}_{\operatorname{Lift}_{Q}}(q)$ converges (uniformly on compact sets) to an analytic function on a neighbourhood of $V_{\sigma, \sigma, I}$ in $V_{\sigma, \sigma}$.

Let $\tilde{Q} \in \tilde{B}_{0}$ be a lift of $Q$ and $\tilde{\sigma}$ the lift of $\sigma$ containing $\tilde{Q}$. Let $u_{1}, u_{2}$ be the primitive generators of $\tilde{\sigma}$ (a basis of $M$ ) such that the orientation of $u_{1}, u_{2}$ agrees with that of $w_{1}, w_{2}$. Let $X_{i}=z^{\tilde{\varphi}_{\tilde{\sigma}}\left(u_{i}\right)}, i=1,2$, be the associated coordinate functions on $V_{\sigma, \sigma, I}$, so that

$$
V_{\sigma, \sigma, I} \subset\left\{\left(X_{1}, X_{2}\right)|| X_{1}|<R| X_{2}|,| X_{2}|<R| X_{1} \mid\right\} \subset\left(\mathbb{G}_{m}\right)_{X_{1}, X_{2}}^{2} \times S_{I}^{\prime} .
$$

For $m \in M$, writing $m=\alpha_{1} u_{1}+\alpha_{2} u_{2}$, we have $z^{\tilde{\varphi} \tilde{\sigma}(m)}=X_{1}^{\alpha_{1}} X_{2}^{\alpha_{2}}$.

As already noted, broken lines $\gamma$ on $B_{0}$ for $q$ with endpoint $Q$ lift uniquely to broken lines on $\tilde{B}_{0}$ for $T^{N}(\tilde{q})$ with endpoint $\tilde{Q}$, for some $N \in \mathbb{Z}$, and the attached monomials are identified. Write $\operatorname{Mono}(\gamma)=a_{\gamma} z^{\tilde{\varphi}_{\tilde{\sigma}}\left(m_{\gamma}\right)}$ and $m_{\gamma}=\alpha_{1} u_{1}+\alpha_{2} u_{2}$. Clearly $\operatorname{Mono}(\gamma)=a_{\gamma} X_{1}^{\alpha_{1}} X_{2}^{\alpha_{2}} \in \mathbb{k}[P]\left[X_{1}^{ \pm 1}, X_{2}^{ \pm 1}\right]$ is an analytic function on $V_{\sigma, \sigma}$. Also write $m_{\gamma}=\mu_{1} w_{1}+\mu_{2} w_{2}$. By Lemma [.12, (1), $\mu_{1}$ and $\mu_{2}$ are bounded below (using the symmetric statement interchanging $w_{1}$ and $w_{2}$ if $\left.T^{N}(\tilde{q}) \in\left\langle\tilde{Q}, w_{1}\right\rangle_{\mathbb{R}_{\geq 0}}\right)$. The points $u_{1}$ and $u_{2}$ are adjacent integral points on the boundary of the infinite convex polytope $\Xi$ with asymptotic directions $w_{1}, w_{2}$. It follows that $w_{1}=\beta_{1} u_{2}+\gamma_{1}\left(u_{1}-u_{2}\right)$ and $w_{2}=\beta_{2} u_{1}+\gamma_{2}\left(u_{2}-u_{1}\right)$, for some $\beta_{1}, \beta_{2}, \gamma_{1}, \gamma_{2}>0$. Hence

$$
\left|z^{\tilde{\varphi} \tilde{\sigma}\left(m_{\gamma}\right)}\right|=\left|X_{1}^{\alpha_{1}} X_{2}^{\alpha_{2}}\right|=\left(\left|X_{2}\right|^{\beta_{1}}\left|X_{1} / X_{2}\right|^{\gamma_{1}}\right)^{\mu_{1}}\left(\left|X_{1}\right|^{\beta_{2}}\left|X_{2} / X_{1}\right|^{\gamma_{2}}\right)^{\mu_{2}} .
$$

Now $\left|X_{1} / X_{2}\right|<R,\left|X_{2} / X_{1}\right|<R$ on $V_{\sigma, \sigma}$. Thus, as we have chosen $\delta$ in Lemma 7.6 so that $0<\delta<\min \left(R^{-\gamma_{1} / \beta_{1}}, R^{-\gamma_{2} / \beta_{2}}\right)$, if $\mu_{1}, \mu_{2}$ are both positive, $\left|z^{\tilde{\varphi}_{\tilde{\sigma}}\left(m_{\gamma}\right)}\right|$ is bounded for $\left|X_{1}\right|,\left|X_{2}\right|<\delta$. On the other hand, suppose $\mu_{1}$, say, is negative. Then if $\left|X_{2}\right|>\delta^{\prime}>0$, we have

$$
\left(\left|X_{2}\right|^{\beta_{1}}\left|X_{1} / X_{2}\right|^{\gamma_{1}}\right)^{\mu_{1}}<\left(\delta^{\prime}\right)^{\beta_{1} \mu_{1}}\left|X_{2} / X_{1}\right|^{-\mu_{1} \gamma_{1}}<\left(\delta^{\prime}\right)^{\beta_{1} \mu_{1}} R^{-\mu_{1} \gamma_{1}} .
$$

Since $\beta_{1}$ and $\gamma_{1}$ are fixed and $\mu_{1}$ is bounded below, the above quantity is bounded. Similarly, if $\mu_{2}$ is negative, $\left(\left|X_{1}\right|^{\beta_{2}}\left|X_{2} / X_{1}\right|^{\gamma_{2}}\right)^{\mu_{2}}$ is bounded provided $\left|X_{1}\right|>\delta^{\prime}>0$. Thus in any event, $\left|z^{\tilde{\varphi} \tilde{\sigma}\left(m_{\gamma}\right)}\right|$ is bounded for $0<\delta^{\prime}<\left|X_{1}\right|,\left|X_{2}\right|<\delta$. (We will only obtain uniform convergence of the series $\operatorname{Lift}_{Q}(q)$ on compact subsets of $V_{\sigma, \sigma}$.) By Lemma 7.8, (3), if $\left|z^{p}\right|<\epsilon<1$ for all $p \in P$ we have $\left|a_{\gamma}\right|=O\left(\epsilon^{|N|}\right)$. By Lemma 7.8, 
(2), the number of broken lines for $T^{N}(\tilde{q})$ is $O\left(|N|^{k}\right)$. Combining, we deduce that $\operatorname{Lift}_{q}(Q)=\sum \operatorname{Mono}(\gamma)$ is convergent on the open analytic subset $V_{\sigma, \sigma}^{\prime}$ of $V_{\sigma, \sigma}$ defined by $\left|X_{1}\right|,\left|X_{2}\right|<\delta$ and $\left|z^{p}\right|<1$ for all $p \in P$, for some $\delta>0$ (independent of $I$ and q). After replacing $X_{J}$ by an analytic neighbourhood of the vertex $s(0) \in X_{J}$, we may assume that $V_{\sigma, \sigma, I} \subset V_{\sigma, \sigma}^{\prime}$.

Proposition 7.11. Let $Q \in B_{0} \backslash \operatorname{Supp}(\mathfrak{D})$ be a point contained in the interior of a maximal cone $\sigma$ of $\Sigma$ and let $\rho$ be an edge of $\sigma$. Consider the formal sum $\operatorname{Lift}_{Q}(q)=$ $\sum \operatorname{Mono}(\gamma)$. For $Q$ sufficiently close to $\rho$ each term of the sum is an analytic function on $V_{\rho, I}$ and the sum defines an analytic function on $V_{\rho, I}$.

Proof. Write $\rho=\rho_{i}$, without loss of generality assume $\sigma=\sigma_{i, i+1}$, so that $\sigma_{i-1, i} \in \Sigma$ is the other maximal cone containing $\rho_{i}$. Let $\tilde{Q}, \tilde{\rho}_{i}, \tilde{\sigma}_{i, i+1}, \tilde{\sigma}_{i-1, i}$ be compatible lifts to $\tilde{B}_{0}$. Let $u_{i}, u_{i-1}, u_{i+1}$ be the primitive generators of $\tilde{\rho}_{i}$ and the remaining edges of $\tilde{\sigma}_{i-1, i}$ and $\tilde{\sigma}_{i, i+1}$, and write $X_{i}, X_{i-1}, X_{i+1}$ for the corresponding coordinates on $V_{\rho, I}$. So

$$
\begin{aligned}
V_{\rho, I} \subset\left\{\left(X_{i-1}, X_{i}, X_{i+1}\right)|| X_{i-1}|<R| X_{i}|,| X_{i+1}|<R| X_{i} \mid\right\} & \subset V\left(X_{i-1} X_{i+1}-z^{\left[D_{\rho}\right]} X_{i}^{-D_{\rho}^{2}} f_{\rho}\right) \\
& \subset \mathbb{A}_{X_{i-1}, X_{i+1}}^{2} \times\left(\mathbb{G}_{m}\right)_{X_{i}} \times S_{I}^{\prime} .
\end{aligned}
$$

Define

$$
\begin{aligned}
V_{\rho}=\left\{\left(X_{i-1}, X_{i}, X_{i+1}\right)|| X_{i-1}|<R| X_{i}|,| X_{i+1}|<R| X_{i} \mid\right\} & \subset V\left(X_{i-1} X_{i+1}-z^{\left[D_{\rho}\right]} X_{i}^{-D_{\rho}^{2}} f_{\rho}\right) \\
& \subset \mathbb{A}_{X_{i-1}, X_{i+1}}^{2} \times\left(\mathbb{G}_{m}\right)_{X_{i}} \times S .
\end{aligned}
$$

We assume that the orientation of $u_{i-1}, u_{i+1}$ is the same as that of $w_{1}, w_{2}$.

We first consider broken lines $\gamma$ lying in the cone generated by $u_{i}$ and $w_{2}$. Write $\operatorname{Mono}(\gamma)=a_{\gamma} z^{\tilde{\varphi}_{\tilde{i}, i+1}\left(m_{\gamma}\right)}$, and $m_{\gamma}=\alpha u_{i}+\alpha_{+} u_{i+1}=\mu_{1} w_{1}+\mu_{2} w_{2}$. By Lemma 7.12, (1), $\left|\mu_{1}\right|$ is bounded, and $\mu_{2}>0$ for all but finitely many $\gamma$. By Lemma 7.12, (2), $\alpha_{+} \geq 0$, so $\operatorname{Mono}(\gamma)=a_{\gamma} X_{i}^{\alpha} X_{i+1}^{\alpha_{+}} \in \mathbb{k}[P]\left[X_{i}^{ \pm 1}, X_{i-1}, X_{i+1}\right]$ is analytic on $V_{\rho}$ for each $\gamma$. In particular we may assume in what follows (discarding finitely many terms $\operatorname{Mono}(\gamma)$ ) that $\mu_{2}>0$. Writing $w_{1}=-\beta_{1} u_{i+1}+\gamma_{1} u_{i}$ and $w_{2}=\beta_{2} u_{i}+\gamma_{2}\left(u_{i+1}-u_{i}\right)$, we have $\beta_{1}, \beta_{2}, \gamma_{1}, \gamma_{2}>0$ and

$$
\left|z^{\tilde{\varphi}_{\tilde{\sigma}}\left(m_{\gamma}\right)}\right|=\left(\left|X_{i+1}\right|^{-\beta_{1}}\left|X_{i}\right|^{\gamma_{1}}\right)^{\mu_{1}}\left(\left|X_{i}\right|^{\beta_{2}}\left|X_{i+1} / X_{i}\right|^{\gamma_{2}}\right)^{\mu_{2}} .
$$

Recall that $\left|X_{i+1} / X_{i}\right|,\left|X_{i-1} / X_{i}\right|<R$ on $V_{\rho}$. Note then that for $0<\delta<R^{-\gamma_{2} / \beta_{2}}$, the second factor on the right is bounded for $\left|X_{i}\right|<\delta$ as we are taking $\mu_{2}>0$. If $\mu_{1}<0$, then we have

$$
\left(\left|X_{i+1}\right|^{-\beta_{1}}\left|X_{i}\right|^{\gamma_{1}}\right)^{\mu_{1}}<R^{-\beta_{1} \mu_{1}}\left|X_{i}\right|^{\left(-\beta_{1}+\gamma_{1}\right) \mu_{1}}
$$


which is bounded for $\delta^{\prime}<\left|X_{i}\right|<\delta$ for any small $\delta^{\prime}>0$. Finally, if $\mu_{1}>0$, we use the equation for $V_{\rho}$, which gives

$$
\left|X_{i+1}\right|^{-1}=\left|X_{i-1}\right| \cdot\left|X_{i}\right|^{D_{\rho}^{2}} \cdot\left|f_{\rho}\right|^{-1} \cdot\left|z^{-\left[D_{\rho}\right]}\right| .
$$

The function $f_{\rho}$ on $V_{\rho}$ restricts to the constant function 1 over $S_{J}$. Hence we may impose the condition $\left|f_{\rho}\right|>\delta^{\prime}$ for any small $\delta^{\prime}>0$. Note that

$$
\left|X_{i+1}\right|^{-\beta_{1} \mu_{1}}=\left(\left|X_{i-1}\right|\left|X_{i}\right|^{D_{\rho}^{2}}\left|f_{\rho}\right|^{-1}\left|z^{-\left[D_{\rho}\right]}\right|\right)^{\beta_{1} \mu_{1}}<R^{\beta_{1} \mu_{1}}\left|X_{i}\right|^{\beta_{1} \mu_{1}\left(1+D_{\rho}^{2}\right)}\left|f_{\rho}\right|^{-\beta_{1} \mu_{1}}\left|z^{\left[D_{\rho}\right]}\right|^{-\beta_{1} \mu_{1}} .
$$

Thus we see that in any event, if $\delta^{\prime}<\left|X_{i}\right|<\delta,\left|f_{\rho}\right|>\delta^{\prime}$ and $\left|z^{p}\right|<\epsilon<1$ for all $p \in P$, then

$$
\left|z^{\tilde{\varphi}_{\tilde{\sigma}}\left(m_{\gamma}\right)}\right| \cdot\left|z^{\left[D_{\rho}\right]}\right|^{c}
$$

is bounded, where $c=\beta_{1} \cdot \sup \left(\left\{\mu_{1}\right\}, 0\right)$ is a constant. Now by Lemma 7.8, (3), again using $\left|z^{p}\right|<\epsilon<1$ for all $p \in P$, then

$$
|\operatorname{Mono}(\gamma)|=\left|a_{\gamma} z^{\tilde{\varphi}_{\tilde{\sigma}}\left(m_{\gamma}\right)}\right|=O\left(\epsilon^{|N|}\right) .
$$

Recall that the number of broken lines for $T^{N}(\tilde{q})$ is $O\left(|N|^{k}\right)$ (Lemma 7.8, (2)). We deduce that the sum $\sum \operatorname{Mono}(\gamma)$ over broken lines $\gamma$ lying in $\left\langle u_{i}, w_{2}\right\rangle_{\mathbb{R}_{\geq 0}}$ is uniformly convergent on compact sets for $\left|X_{i}\right|<\delta, f_{\rho} \neq 0$, and $\left|z^{p}\right|<1$ for all $p \in P$, where $\delta>0$ is independent of $I$ and $q$.

Symmetrically, if we choose a basepoint $Q^{\prime} \in \sigma_{i-1, i}$ sufficiently close to $\rho$, we can use the same argument for broken lines lying in $\left\langle u_{i}, w_{1}\right\rangle_{\mathbb{R}_{\geq 0}}$ ending at $\tilde{Q}^{\prime}$. Such a broken line will have $\operatorname{Mono}(\gamma)=a_{\gamma} X_{i-1}^{\alpha-} X_{i}^{\alpha}$ with $\alpha_{-} \geq 0$. The same argument as above shows that the sum $\sum \operatorname{Mono}(\gamma)$ over all such broken lines $\gamma$ is uniformly convergent on compact sets for $\left|X_{i}\right|<\delta, f_{\rho} \neq 0$, and $\left|z^{p}\right|<1$ for all $p \in P$, where $\delta>0$ is again independent of $I$ and $q$. However, the statement we are trying to prove involves the lift at $Q$, not $Q^{\prime}$. For this purpose, we will omit terms coming from broken lines with $\alpha_{-}=0$ from the above sum, as such terms will also arise in the above analysis at $\tilde{Q}$.

To deal with this issue, note that given such a broken line $\gamma$ ending at $\tilde{Q}^{\prime}$ with $\alpha_{-}>0$, we get a finite number of broken lines $\gamma_{j}, 1 \leq j \leq s$, ending at a point $\tilde{Q}^{\prime \prime} \in \tilde{\sigma}_{i, i+1}$ sufficiently close to $\tilde{\rho}_{i}$ as follows. Extend $\gamma$ until it reaches $\tilde{\rho}_{i}$ (which can be done since $\left.\alpha_{-}>0\right)$. Then applying the automorphism associated to crossing $\tilde{\rho}_{i}$ to $\operatorname{Mono}(\gamma)$ gives $a_{\gamma} X_{i-1}^{\alpha_{-}} X_{i}^{\alpha} f_{\rho}^{\alpha_{-}}$, which we write as $\sum_{j=1}^{s} a_{\gamma_{j}} X_{i-1}^{\alpha_{-}} X_{i}^{\alpha_{j}}$. The $j^{\text {th }}$ monomial in the sum gives a new broken line $\gamma_{j}$ bending at $\tilde{\rho}_{i}$ (unless $\alpha_{j}=\alpha$, in which case we just extend the final line segment of $\gamma$ ), with new attached monomial $a_{\gamma_{j}} X_{i-1}^{\alpha-} X_{i}^{\alpha_{j}}=a_{\gamma_{j}} z^{\tilde{\sigma}_{\tilde{\sigma}_{i, i+1}}\left(m_{\gamma_{j}}\right)}$. As long as $\tilde{Q}^{\prime \prime}$ lies in the cone generated by $\rho_{i}$ and $-m_{\gamma_{j}}$, by extending or shortening the last line segment of $\gamma_{j}$ and applying a homothety, one can obtain a broken line $\gamma_{j}$ ending at $\tilde{Q}^{\prime \prime}$. Thus for $\tilde{Q}^{\prime \prime}$ sufficiently close to $\tilde{\rho}_{i}$, we obtain broken lines $\gamma_{1}, \ldots, \gamma_{s}$ ending at $\tilde{Q}^{\prime \prime}$. However, the choice of $\tilde{Q}^{\prime \prime}$ may depend on $\gamma$. To see there is a choice of $\tilde{Q}^{\prime \prime}$ which 
works for all $\gamma$, note that for all but a finite number of $\gamma, m_{\gamma}$ lies in the half-space $\mathbb{R}_{\geq 0} \cdot w_{1}+\mathbb{R} \cdot w_{2}$ by Lemma 7.12, (1). Further, since $\gamma_{j}$ bends at $\tilde{\rho}_{i}, m_{\gamma} \in \mathbb{Z} \cdot u_{i}+\mathbb{Z}_{<0} \cdot u_{i+1}$. Thus $m_{\gamma}=\beta u_{i}+\beta_{+} u_{i+1}$ for $\beta>0, \beta_{+}<0$, and $m_{\gamma_{j}}=(\beta-l) u_{i}+\beta_{+} u_{i+1}$ for some $l>0$ with a bound only depending on $f_{\rho_{i}} \bmod I$. ¿From this it follows that there cannot be a sequence of $\gamma$ and $\gamma_{j}$ constructed from $\gamma$ as above so that the cones generated by $u_{i}$ and $-m_{\gamma_{j}}$ get smaller and smaller.

Thus we see that taking $\tilde{Q}$ sufficiently close to $\tilde{\rho}_{i}$, the broken lines ending at $\tilde{Q}^{\prime}$ contained in $\left\langle w_{1}, \tilde{Q}^{\prime}\right\rangle_{\mathbb{R}_{\geq 0}}$ with $\alpha_{-}>0$ give in the above fashion broken lines ending at $\tilde{Q}$ contained in $\left\langle w_{1}, \tilde{Q}\right\rangle_{\mathbb{R}_{>0}}$, and every such broken line ending at $\tilde{Q}$ clearly arises in this way. Furthermore, there are no broken lines contained in $\left\langle w_{1}, \tilde{Q}\right\rangle_{\mathbb{R}_{\geq 0}}$ with $\alpha_{-}=0$, and thus all broken lines ending at $\tilde{Q}$ have been accounted for.

Now consider again a broken line $\gamma$ contained in $\left\langle w_{1}, \tilde{Q}^{\prime}\right\rangle_{\mathbb{R}_{\geq 0}}$ with $\alpha_{-}>0$. By construction, $\sum_{j=1}^{s} \operatorname{Mono}\left(\gamma_{j}\right)=\operatorname{Mono}(\gamma) f_{\rho}^{\alpha_{-}}$. We wish to understand the contribution of $\sum_{j=1}^{s} \operatorname{Mono}\left(\gamma_{j}\right)$ to $\operatorname{Lift}_{Q}(q)$, and to do so, we write $\operatorname{Mono}\left(\gamma_{j}\right)$ in terms of $X_{i}, X_{i+1}$ using the relation $X_{i-1} X_{i+1}=z^{\left[D_{\rho}\right]} X_{i}^{-D_{\rho}^{2}}$ in $\mathbb{k}\left[P_{\tilde{\varphi}_{\tilde{\rho}}}\right]$ (see Proposition 2.5 ). So we can write

$$
\sum_{j} \operatorname{Mono}\left(\gamma_{j}\right)=a_{\gamma} z^{\alpha_{-}\left[D_{\rho}\right]} X_{i+1}^{-\alpha_{-}} X_{i}^{-\alpha_{-} D_{\rho}^{2}+\alpha} f_{\rho}^{\alpha_{-}} .
$$

This defines a (possibly rational) function on $V_{\rho}$. On the other hand, $\operatorname{Mono}(\gamma)=$ $a_{\gamma} X_{i-1}^{\alpha_{-}} X_{i}^{\alpha}$ defines a holomorphic function on $V_{\rho}$, and using the equation

$$
X_{i-1} X_{i+1}=z^{\left[D_{\rho}\right]} X_{i}^{-D_{\rho}^{2}} f_{\rho}
$$

which is satisfied on $V_{\rho}$, we have

$$
\operatorname{Mono}(\gamma)=a_{\gamma} z^{\alpha_{-}\left[D_{\rho}\right]} X_{i+1}^{-\alpha_{-}} X_{i}^{-\alpha_{-} D_{\rho}^{2}+\alpha} f_{\rho}^{\alpha_{-}}
$$

as a function on $V_{\rho}$. Thus we see that $\operatorname{Mono}(\gamma)$ and $\sum_{j} \operatorname{Mono}\left(\gamma_{j}\right)$ coincide as functions on $V_{\rho}$, in the above interpretation. (Essentially we are just using the fact that the relation (7.6) encodes the automorphism associated to crossing $\rho$ ). Thus the fact that $\sum_{\gamma} \operatorname{Mono}(\gamma)$ defines an analytic function on $V_{\rho, I}$ for broken lines $\gamma$ ending at $\tilde{Q}^{\prime}$ contained in $\left\langle u_{i}, w_{1}\right\rangle_{\mathbb{R}_{\geq 0}}$ implies that the $\operatorname{sum} \sum_{\gamma} \operatorname{Mono}(\gamma)$ over all broken lines $\gamma$ ending at $\tilde{Q}$ and contained in $\left\langle\tilde{Q}, w_{1}\right\rangle_{\mathbb{R}_{\geq 0}}$ is analytic. This $\operatorname{implies}_{\operatorname{Lift}_{Q}}(q)$ is analytic on $V_{\rho, I}$.

Lemma 7.12. Let $\tilde{Q} \in \tilde{B}_{0} \backslash \operatorname{Supp}(\tilde{\mathfrak{D}})$ be a point contained in the interior of a maximal cone $\tilde{\sigma} \in \tilde{\Sigma}$. Consider broken lines $\gamma$ on $\tilde{B}_{0}$ for $T^{N}(\tilde{q})$ with endpoint $\tilde{Q}$, for all $N \in \mathbb{Z}$ such that $T^{N}(\tilde{q}) \in\left\langle\tilde{Q}, w_{2}\right\rangle_{\mathbb{R}_{\geq 0}}$. Write $\operatorname{Mono}(\gamma)=a_{\gamma} z^{\tilde{\varphi}_{\tilde{\sigma}}\left(m_{\gamma}\right)}$, and $m_{\gamma}=\mu_{1} w_{1}+\mu_{2} w_{2}$.

(1) $\left|\mu_{1}\right|$ is bounded, and $\mu_{2}$ is positive for all but finitely many $\gamma$. In particular, $\mu_{1}, \mu_{2}$ are bounded below. 
(2) Let $u_{1}, u_{2}$ be generators of $\tilde{\sigma}$ with the same orientation as $w_{1}, w_{2}$. Then for $\tilde{Q}$ sufficiently close to $\tilde{\rho}:=\mathbb{R}_{\geq 0} \cdot u_{1}, m_{\gamma}$ lies in the half space $\mathbb{R} \cdot u_{1}+\mathbb{R}_{\geq 0} \cdot u_{2}$ for each $\gamma$.

Proof. (1) We may assume the lift $\tilde{q}$ of $q$ is chosen so that $T^{N}(\tilde{q}) \in\left\langle\tilde{Q}, w_{2}\right\rangle_{\mathbb{R}_{\geq 0}}$ if and only if $N \geq 0$. Note that the rays spanned by $w_{1}, w_{2}$ are irrational so $\mu_{1}, \mu_{2} \neq 0$. Suppose for a contradiction that there is an infinite sequence of broken lines $\gamma$ such that $m_{\gamma}=\mu_{1} w_{1}+\mu_{2} w_{2}$ with $\mu_{2}<0$. Each broken line has at most $k$ bends and there are a finite number of $\Gamma$-orbits of possible changes $\alpha \in M$ of the derivative of $\gamma$ at a bend; see Lemma 7.8 and its proof. So, passing to a subsequence, we may assume that the bends (in order of increasing $t \in(-\infty, 0]$ ) of each $\gamma$ are of types $T^{s_{1}} \alpha_{1}, \ldots, T^{s_{l}} \alpha_{l}$ for some fixed $\alpha_{1}, \ldots, \alpha_{l} \in M, l \leq k$, and $N \geq s_{1} \geq s_{2} \geq \cdots \geq s_{l} \geq 0$ (depending on $\gamma$ ). Say $N-s_{i}$ is bounded for $i \leq l^{\prime}$ and unbounded otherwise. Passing to a subsequence, we may assume that $N-s_{i}$ is constant for $i \leq l^{\prime}$. Let $\gamma^{\prime}$ be the broken line obtained by truncating $\gamma$ after the first $l^{\prime}$ bends, and moving by a homothety so that (extending its final line segment) $\gamma^{\prime}$ ends at $\tilde{Q}$. Then $m_{\gamma^{\prime}}=T^{N}(m)$ for some fixed $m \in M$. Furthermore, $m_{\gamma}$ is obtained from $m_{\gamma^{\prime}}$ by adding a positive linear combination of $w_{1}$ and $w_{2}$. But since $\mu_{2}<0$ and $w_{1}, w_{2}$ are eigenvectors of $T$ with eigenvalues $\lambda^{-1}, \lambda$, it follows that we must have $m=\nu_{1} w_{1}+\nu_{2} w_{2}$ with $\nu_{2}<0$. But then for sufficiently large $N, T^{N}(m)$ does not lie in the half-space $\mathbb{R} \cdot \tilde{Q}+\mathbb{R}_{\geq 0} \cdot w_{2}$. This is a contradiction because $m_{\gamma^{\prime}}$ always lies in this half-space.

To see that $\left|\mu_{1}\right|$ is bounded, recall that $m_{\gamma}=T^{N}(\tilde{q})-\sum_{i=1}^{l} T^{s_{i}} \alpha_{i}$ where $0 \leq l \leq k$, $0 \leq s_{i} \leq N$, and the $\alpha_{i}$ are selected from a finite set. Now since $T w_{1}=\lambda^{-1} w_{1}$ it follows that $\left|\mu_{1}\right|$ is bounded.

(2) Let $\mathfrak{u}$ be the connected component of $\tilde{B}_{0} \backslash \operatorname{Supp}_{I}(\tilde{\mathfrak{D}})$ contained in $\tilde{\sigma}$ and containing $\tilde{\rho}$ in its closure. Let $\tilde{Q}^{\prime} \in \mathfrak{u}$ be a point such that $\tilde{Q}^{\prime} \in\langle\tilde{\rho}, \tilde{Q}\rangle_{\mathbb{R}_{\geq 0}}$. Then if $T^{N}(\tilde{q}) \in$ $\left\langle\tilde{Q}, w_{2}\right\rangle_{\mathbb{R}_{>0}}$ and $\gamma^{\prime}$ is a broken line for $T^{N}(\tilde{q})$ with endpoint $\tilde{Q}^{\prime}$, we obtain a broken line $\gamma$ for $T^{N}(\tilde{q})$ with endpoint $\tilde{Q}$ and $m_{\gamma}=m_{\gamma^{\prime}}$ as follows. First apply a homothety to obtain a broken line passing through $\tilde{Q}$, then truncate at $\tilde{Q}$. This gives an injective map between the set of broken lines for $T^{N}(\tilde{q}) \in\left\langle\tilde{Q}, w_{2}\right\rangle_{\mathbb{R}_{\geq 0}}$ ending at $\tilde{Q}^{\prime}$ with the set of such broken lines ending at $\tilde{Q}$.

Now suppose $\gamma$ is a broken line for $T^{N}(\tilde{q})$ with endpoint $\tilde{Q}$ and $m_{\gamma}$ not lying in the half-space $\mathbb{R} \cdot u_{1}+\mathbb{R}_{\geq 0} \cdot u_{2}$. Since $m_{\gamma}$ lies in the half-space $\mathbb{R} \cdot \tilde{Q}+\mathbb{R}_{\geq 0} \cdot w_{2}$ we find $m_{\gamma}$ lies in the cone generated by $-\tilde{Q},-u_{1}$. In particular, $m_{\gamma}$ does not lie in the half-space $\mathbb{R} \cdot w_{1}+\mathbb{R}_{\geq 0} w_{2}$, so by (1) there are only finitely many such $\gamma$. Now by the above construction it follows that for $\tilde{Q}$ sufficiently close to $\tilde{\rho}$ there are none.

7.4. Smoothness. We will now complete the proof of: 
Theorem 7.13. (Looijenga's conjecture) Suppose that $\mathbb{k}=\mathbb{C}$ and the intersection matrix $\left(D_{i} \cdot D_{j}\right)_{1 \leq i, j \leq n}$ is negative definite, so that $D \subset Y$ can be contracted to a cusp singularity $q \in Y^{\prime}$. Then the dual cusp to $q \in Y^{\prime}$ is smoothable.

We continue to work with the setup at the beginning of $\$ 7.2$, with $L, \sigma_{P}, P, \mathfrak{m}$ and $J$ as given there. By Theorem 7.7, if $I$ is a monomial ideal with $\sqrt{I}=J$, we obtain a deformation $X_{I} \rightarrow S_{I}^{\prime}$ of $X_{J} \rightarrow S_{J}^{\prime}$. For the remainder of the section, we shall write $S_{I}$ for $S_{I}^{\prime}$. Let $\mathfrak{f}_{J}: \mathfrak{X}_{J} \rightarrow \mathfrak{S}_{J}$ denote the formal deformation determined by the deformations $X_{J^{N+1}} \rightarrow S_{J^{N+1}}$ for $N \geq 0$. Thus, $\mathfrak{S}_{J}$ is the formal complex analytic space obtained as the completion of $S$ along $S_{J}, \mathfrak{X}_{J}$ is a formal complex analytic space, and $\mathfrak{X}_{J} \rightarrow \mathfrak{S}_{J}$ is an adic flat morphism. We similarly have the family $\mathfrak{X}_{\mathfrak{m}} \rightarrow \mathfrak{S}_{\mathfrak{m}}$ of formal schemes already studied in 44 . We refer to [G60] and [B78] for background on formal schemes and formal complex analytic spaces.

We have a section $s: S_{J} \rightarrow X_{J}$ such that, for $t \in S_{J}$ general, the point $s(t) \in X_{J, t}$ on the fibre is the cusp. We write $X_{J}^{o}:=X_{J} \backslash s\left(S_{J}\right) \subset X_{J}$ and $X_{I}^{o} \subset X_{I}, \mathfrak{X}_{J}^{o} \subset \mathfrak{X}_{J}$ for the induced open embeddings.

Let $Z_{I}:=\operatorname{Sing}\left(f_{I}\right) \subset X_{I}$ denote the singular locus of $f_{I}: X_{I} \rightarrow S_{I}$, see DefinitionLemma 4.1. Thus $Z_{I} \subset X_{I}$ is a closed embedding of schemes or complex analytic spaces. Since the singular locus is compatible with base-change, the singular loci $Z_{J^{n}} \subset X_{J^{n}}$ determine a closed embedding $\mathfrak{Z}_{J} \subset \mathfrak{X}_{J}$ which we refer to as the singular locus of $\mathfrak{f}_{J}: \mathfrak{X}_{J} \rightarrow \mathfrak{S}_{J}$.

Lemma 7.14. In the above situation, there exists $0 \neq g \in \mathbb{k}[P]$ such that $\operatorname{Supp}\left(g \cdot \mathcal{O}_{\mathfrak{Z}_{J}}\right)$ is contained in $s\left(S_{J}\right)$. In particular, $\mathfrak{f}_{J *}\left(g \cdot \mathcal{O}_{\mathfrak{Z}_{J}}\right)$ is a coherent sheaf on $\mathfrak{S}_{J}$.

Proof. The proof is essentially the same as that of Lemma 4.5. Let $\mathfrak{U}_{i, J}$ be defined as in (4.1). Then $\mathfrak{X}_{J}^{o}$ is a union of open subspaces $\mathfrak{V}_{i, J}, i=1, \ldots, n$, such that $\mathfrak{V}_{i, J}$ is an analytic open subspace of $\mathfrak{U}_{i, J}$ for each $i$. We then take $g=a_{1} \cdots a_{n}$ as in the proof of Lemma 4.5, so that $\operatorname{Supp}\left(g \cdot \mathcal{O}_{\mathfrak{Z}_{J}}\right)$ is contained in $s\left(S_{J}\right)$. So the support of $g \cdot \mathcal{O}_{\mathfrak{Z}_{J}}$ is a closed subset of $s\left(S_{J}\right)$, hence proper over $S_{J}$. It follows that $\mathfrak{f}_{J *}\left(g \cdot \mathcal{O}_{\mathfrak{Z}_{J}}\right)$ is coherent by [B78, 3.1.

Let $u(J)$ denote the natural map

$$
u(J): \mathcal{O}_{\mathfrak{S}_{J}} \rightarrow \mathfrak{f}_{J *}\left(\mathcal{O}_{\mathfrak{Z}_{J}}\right),
$$

and $u(\mathfrak{m})$ similarly the natural map

$$
u(\mathfrak{m}): \mathcal{O}_{\mathfrak{S}_{\mathfrak{m}}} \rightarrow \mathfrak{f}_{\mathfrak{m} *}\left(\mathcal{O}_{\mathfrak{Z}_{\mathfrak{m}}}\right) .
$$

Lemma 7.15. $u(J)$ is injective if and only if $u(\mathfrak{m})$ is injective. 
Proof. Let $0 \neq g \in \mathbb{k}[P]$ be the element given by Lemma 7.14 Let $\mathcal{K}_{J}$ be the kernel of $u(J)$ and $\mathcal{K}_{J}^{\prime}$ the kernel of $g \cdot u(J)$. Thus $\mathcal{K}_{J}, \mathcal{K}_{J}^{\prime}$ are ideal sheaves in $\mathcal{O}_{\mathfrak{G}_{J}}$ and $g \cdot \mathcal{K}_{J}^{\prime} \subset \mathcal{K}_{J} \subset \mathcal{K}_{J}^{\prime}$. The local rings of $\mathfrak{S}_{J}$ are domains by Lemma 7.16, so $\mathcal{K}_{J}=0$ if and only if $\mathcal{K}_{J}^{\prime}=0$. The sheaf $\mathcal{K}_{J}^{\prime}$ is coherent because the image of $g \cdot u(J)$ is contained in the coherent subsheaf $\mathfrak{f}_{J *}\left(g \cdot \mathcal{O}_{\mathfrak{J}_{J}}\right) \subset \mathfrak{f}_{J *} \mathcal{O}_{\mathfrak{Z}_{J}}$.

We claim that the natural map

$$
\mathcal{K}_{J}^{\prime} \otimes_{\mathcal{O}_{\mathfrak{G}_{J}}} \mathcal{O}_{\mathfrak{S}_{\mathfrak{m}}} \rightarrow \mathcal{K}_{\mathfrak{m}}^{\prime}
$$

is an isomorphism. Let $z \in \mathfrak{S}_{\mathfrak{m}}$ be the unique point, coinciding with the zerodimensional torus orbit of $S$, and let $\hat{\mathcal{O}}_{S, z}$ denote the completion of $\mathcal{O}_{S, z}$ at its maximal ideal. Note that $\hat{\mathcal{O}}_{S, z}$ coincides with $\mathcal{O}_{\mathfrak{S}_{\mathfrak{m}}, z}$ and the completion of $\mathcal{O}_{\mathfrak{S}_{J}, z}$ at its maximal ideal. It suffices to show that the map

$$
\mathcal{K}_{J, z}^{\prime} \otimes_{\mathcal{O}_{\mathfrak{S}_{J}, z}} \hat{\mathcal{O}}_{S, z} \rightarrow \mathcal{K}_{\mathfrak{m}, z}^{\prime}
$$

is an isomorphism. We have an exact sequence of coherent sheaves

$$
0 \rightarrow \mathcal{K}_{J}^{\prime} \rightarrow \mathcal{O}_{\mathfrak{S}_{J}} \rightarrow \mathfrak{f}_{J *}\left(g \cdot \mathcal{O}_{\mathfrak{Z}_{J}}\right)
$$

and so an exact sequence of $\hat{\mathcal{O}}_{S, z}$-modules

$$
0 \rightarrow \mathcal{K}_{J, z}^{\prime} \otimes \hat{\mathcal{O}}_{S, z} \rightarrow \hat{\mathcal{O}}_{S, z} \rightarrow \mathfrak{f}_{J *}\left(g \cdot \mathcal{O}_{\mathfrak{J}_{J}}\right)_{z} \otimes \hat{\mathcal{O}}_{S, z} .
$$

Now

$$
\mathfrak{f}_{J *}\left(g \cdot \mathcal{O}_{\mathfrak{Z}_{J}}\right)_{z} \otimes \hat{\mathcal{O}}_{S, z}=\left(g \cdot \mathcal{O}_{\mathfrak{Z}_{J}}\right)_{s(z)} \otimes \hat{\mathcal{O}}_{S, z}=\left(\widehat{g \cdot \mathcal{O}_{\mathfrak{Z}_{J}}}\right)_{s(z)}=g \cdot \hat{\mathcal{O}}_{\mathfrak{Z}_{J}, s(z)}
$$

where the hats denote completion with respect to the maximal ideal of $\mathcal{O}_{\mathfrak{S}_{J}, z}$. Thus $\mathcal{K}_{J, z}^{\prime} \otimes \hat{\mathcal{O}}_{S, z}$ is the kernel of the map

$$
\hat{\mathcal{O}}_{S, z} \rightarrow g \cdot \hat{\mathcal{O}}_{\mathfrak{Z}_{J}, s(z)} .
$$

By the base-change property for the singular locus, this map coincides with the corresponding map for $\mathfrak{m}$. This proves the claim.

The support of the ideal sheaf $\mathcal{K}_{J}^{\prime}$ is either empty or $S_{J}$ (because the local rings of $\mathfrak{S}_{J}$ are domains and $S_{J}$ is connected). So $\mathcal{K}_{J}^{\prime}=0$ if and only if $\mathcal{K}_{\mathfrak{m}}^{\prime}=0$ by the claim.

Lemma 7.16. The local rings of $\mathfrak{S}_{J}$ are integral domains.

Proof. The completion of the local ring of $\mathfrak{S}_{J}$ at a point $z \in S_{J}$ is identified with the completion of the local ring of the toric variety $S$ at $z$. By Serre's criterion for normality, the completion of a normal Noetherian ring at a maximal ideal is a local normal Noetherian ring; in particular, it is a domain. Since $\mathcal{O}_{\mathfrak{S}_{J}, z}$ is a local Noetherian ring, it is contained in its completion. We deduce that $\mathcal{O}_{\mathfrak{S}_{J}, z}$ is a domain. 
Proof of Theorem 7.13. Let $f: Y \rightarrow Y^{\prime}$ be the contraction of $D \subset Y$. We may assume $f$ is the minimal resolution of $Y^{\prime}$. We may further assume $n \geq 3$. Indeed, the embedding dimension of the dual cusp equals $\max (n, 3)$ by [N80, Corollary 7.8, p. 232 and [KM98], Theorem 4.57, p. 143, see also [L81], pg. 307. So in particular for $n \leq 3$ the dual cusp is a hypersurface and thus smoothable. Let $L$ be a nef divisor on $Y$ such that $\mathrm{NE}(Y)_{\mathbb{R}_{\geq 0}} \cap L^{\perp}=\left\langle D_{1}, \ldots, D_{n}\right\rangle_{\mathbb{R}_{\geq 0}}$. Let $\sigma_{P} \subset A_{1}(Y, \mathbb{R})$ be a strictly rational polyhedral cone containing $\mathrm{NE}(Y)$ such that $\sigma_{P} \cap L^{\perp}$ is a face of $\sigma_{P}$. Let $P=\sigma_{P} \cap A_{1}(Y, \mathbb{Z})$ and $J=P \backslash P \cap L^{\perp}$. By Lemma 7.15 and Theorem 4.6, $u(J)$ is not injective.

Let $x \in S_{J}$ be a point lying in the interior of the toric variety $S_{J}$ and $h \in \mathcal{O}_{\mathfrak{S}_{J}, x}$ a nonzero element of the kernel of $u(J)$ near $x$. By Lemma 7.17 there is a morphism

$$
v: \operatorname{Spec} \mathbb{C}[t] /\left(t^{N+1}\right) \rightarrow \mathfrak{S}_{J}
$$

taking the unique point of the domain to $x$ and $0 \neq v^{*}(h) \in \mathbb{C}[t] /\left(t^{N+1}\right)$. Let $Y / \operatorname{Spec}\left(\mathbb{C}[t] /\left(t^{N+1}\right)\right)$ be the pullback of $\mathfrak{X}_{J} / \mathfrak{S}_{J}$ by $v$ and $Z \subset Y$ its singular locus. Then $Y / \operatorname{Spec}\left(\mathbb{C}[t] /\left(t^{N+1}\right)\right)$ is a deformation of the dual cusp singularity. Furthermore, $\mathcal{O}_{Z}$ is annihilated by $v^{*}(h)$, and the ideal generated by $v^{*}(h)$ must contain $t^{N}$, so $\mathcal{O}_{Z}$ is annihilated by $t^{N}$. By [A76], Theorem 5.1, there is an algebraic finite type deformation $Y^{\prime} / \operatorname{Spec} \mathbb{C}[t]$ whose restriction to $\operatorname{Spec}\left(\mathbb{C}[t] /\left(t^{N+1}\right)\right)$ is locally analytically isomorphic to $Y / \operatorname{Spec}\left(\mathbb{C}[t] /\left(t^{N+1}\right)\right)$. Let $Z^{\prime} \subset Y^{\prime}$ denote the singular locus of $Y^{\prime} / \operatorname{Spec} \mathbb{C}[t]$. Then $\mathcal{O}_{Z^{\prime}}$ is a finite $\mathbb{C}[t]$-module because the fibre $Y_{0}^{\prime}$ has an isolated singularity (using [Ma89], Theorem 8.4, p. 58). Now $\mathcal{O}_{Z}=\mathcal{O}_{Z^{\prime}} / t^{N+1} \mathcal{O}_{Z^{\prime}}$ and $t^{N} \mathcal{O}_{Z}=0$, so $t^{N} \mathcal{O}_{Z^{\prime}}=t^{N+1} \mathcal{O}_{Z^{\prime}}$ and thus $t^{N} \mathcal{O}_{Z^{\prime}}=0$ by Nakayama's lemma. Hence the general fibre of $Y^{\prime} /$ Spec $\mathbb{C}[t]$ is smooth, and $Y^{\prime} /$ Spec $\mathbb{C}[t]$ is a smoothing of the dual cusp.

Lemma 7.17. Let $A$ be the completion of a finitely generated normal Cohen-Macaulay $\mathbb{C}$-algebra at a maximal ideal. Let $0 \neq a \in A$. Then there exists $N \geq 0$ and a $\mathbb{C}$-algebra map $f: A \rightarrow \mathbb{C}[t] /\left(t^{N+1}\right)$ such that $f(a) \neq 0$.

Proof. Extend $a$ to a regular sequence $a, t_{1}, \ldots, t_{r}$ of length $\operatorname{dim} A$. Then the normalization of $A /\left(t_{1}, \ldots, t_{r}\right)$ is a finite direct sum of copies of $\mathbb{C}[t]$. Now the result is clear.

\section{REFERENCES}

[AC11] D. Abramovich, Q. Chen, Stable logarithmic maps to Deligne-Faltings pairs II, arXiv:1102.4531.

[A02] V. Alexeev, Complete moduli in the presence of semiabelian group action, Ann. of Math. (2) 155 (2002), no. 3, 611-708.

[A98] K. Altmann, $P$-resolutions of cyclic quotients from the toric viewpoint, in Singularities (Oberwolfach, 1996), 241-250, Progr. Math. 162, Birkhäuser, 1998. 
[A76] M. Artin, Lectures on deformations of singularities, Lectures on Mathematics and Physics 54, Tata Inst. Fund. Res., 1976.

[A07] D. Auroux, Mirror symmetry and T-duality in the complement of an anticanonical divisor, J. Gökova Geom. Topol. 1 (2007), 51-91.

[AMRT75] A. Ash, D. Mumford, M. Rapoport, Y. Tai, Smooth compactification of locally symmetric varieties, Math. Sci. Press, 1975.

[B78] J. Bingener, Über formale komplexe Räume, Manuscripta Math. 24 (1978), no. 3, 253-293.

[C1869] A. Cayley, A Memoir on Cubic Surfaces, Philosophical Transactions of the Royal Society of London 159 (1869), 231-326.

[CPS] M. Carl, M. Pumperla, and B. Siebert, A tropical view of Landau-Ginzburg models, available at http://www.math.uni-hamburg.de/home/siebert/preprints/LGtrop.pdf

[CO06] C.-H. Cho, Y.-G. Oh, Floer cohomology and disc instantons of Lagrangian torus fibers in Fano toric manifolds, Asian J. Math. 10, (2006), 773-814.

[E95] D. Eisenbud, Commutative algebra with a view toward Algebraic Geometry, Graduate Texts in Mathematics 150, Springer-Verlag, New York, 1995.

[FG09] V. Fock and A. Goncharov, Cluster ensembles, quantization and the dilogarithm, Ann. Sci.Éc. Norm. Supér. (4) 42 (2009), no. 6, 865-930.

[FM83] R. Friedman and R. Miranda, Smoothing cusp singularities of small length, Math. Ann. 263 (1983), no. 2, 185-212.

[FP84] R. Friedman and H. Pinkham, Smoothings of cusp singularities via triangle singularities. With an appendix by H. Pinkham, Compositio Math. 53 (1984), no. 3, 303-324.

[F75] A. Fujiki, On the blowing down of analytic spaces, Publ. Res. Inst. Math. Sci. 10 (1974/75), 473-507.

[Giv] A. Givental, Homological geometry. I. Projective hypersurfaces. Selecta Math. 1 (1995), 325-345.

[G09] M. Gross, Mirror symmetry for $\mathbb{P}^{2}$ and tropical geometry, Adv. Math., 224 (2010), 169-245.

[G11] M. Gross, Tropical geometry and mirror symmetry, CBMS Regional Conf. Ser. in Math. 114, A.M.S., 2011.

[GHK12] M. Gross, P. Hacking, S. Keel, Moduli of surfaces with anti-canonical cycle, to appear in Comp. Math., preprint, 2012.

[GHK13] M. Gross, P. Hacking, S. Keel, Birational geometry of cluster algebras, to appear in Algebraic Geometry, preprint, 2013.

[GHKK] M. Gross, P. Hacking, S. Keel, M. Kontsevich, Canonical bases for cluster algebras, preprint, 2014.

[GHKII] M. Gross, P. Hacking, S. Keel, Mirror symmetry for log Calabi-Yau surfaces II, in preparation.

[GHKS] M. Gross, P. Hacking, S. Keel, B. Siebert, Theta functions on varieties with effective anticanonical class, in preparation.

[K3] M. Gross, P. Hacking, S. Keel, B. Siebert, Theta functions for K3 surfaces, in preparation.

[GPS09] M. Gross, R. Pandharipande, B. Siebert, The tropical vertex, Duke Math. J. 153, (2010), $197-362$.

[GS06] M. Gross, B. Siebert, Mirror symmetry via logarithmic degeneration data, I. J. Differential Geom., 72, (2006) 169-338. 
[GS07] M. Gross, B. Siebert, From real affine geometry to complex geometry, Annals of Mathematics, 174, (2011), 1301-1428.

[GS08] M. Gross, B. Siebert, An invitation to toric degenerations, Surveys in differential geometry. Volume XVI. Geometry of special holonomy and related topics, 43-78, Surv. Differ. Geom., 16, Int. Press, Somerville, MA, 2011.

[GS11] M. Gross, B. Siebert, Logarithmic Gromov-Witten invariants, J. Amer. Math. Soc. 26 (2013), $451-510$.

[GSTheta] M. Gross, B. Siebert, Theta functions and mirror symmetry, arXiv:1204.1991 [math.AG].

[G60] A. Grothendieck, Éléments de géométrie algébrique I. Le langage des schémas, Inst. Hautes Études Sci. Publ. Math. No. 4.

[G61] A. Grothendieck, Éléments de géométrie algébrique III, Étude cohomologique des faisceaux cohérents I, Inst. Hautes Études Sci. Publ. Math. No. 11 (1961).

[H04] P. Hacking, Compact moduli of plane curves, Duke Math. J. 124 (2004), no. 2, 213-257.

[Hi73] F. Hirzebruch, Hilbert modular surfaces, Enseignement Math., 19, (1973), 183-281.

[H77] R. Hartshorne, Algebraic geometry, Grad. Texts in Math. 52, Springer, 1977.

[IP03] E.-N. Ionel, T. Parker, Relative Gromov-Witten invariants, Ann. of Math. 157 (2003), 45-96.

[KM98] J. Kollár, S. Mori, Birational geometry of algebraic varieties, Cambridge Tracts in Math. 134. C.U.P., 1998.

[KS06] M. Kontsevich, Y. Soibelman: Affine structures and non-Archimedean analytic spaces, in: The unity of mathematics (P. Etingof, V. Retakh, I.M. Singer, eds.), 321-385, Progr. Math. 244, Birkhäuser 2006.

[L02] S. Lang, Algebra, 3rd ed., Grad. Texts in Math. 211, Springer, 2002.

[L73] H. Laufer, Taut two-dimensional singularities, Math. Ann. 205 (1973), 131-164.

[Li00] J. Li, Stable morphisms to singular schemes and relative stable morphisms, J. Diff. Geom. 57, (2000), 509-578.

[Li02] J. Li, A degeneration formula of GW-invariants, J. Diff. Geom., 60, (2002) 199-293.

[LT98] J. Li, G. Tian, Virtual moduli cycles and Gromov-Witten invariants of algebraic varieties, J. of the AMS, 11, (1998), 119-174.

[LR01] A.-M. Li, Y. Ruan, Symplectic surgery and Gromov-Witten invariants of Calabi-Yau 3-folds, I, Invent. Math. 145 (2001), 151-218.

[L76] E. Looijenga, Root systems and elliptic curves, Invent. Math. 38 (1976), 17-32.

[L81] E. Looijenga, Rational surfaces with an anticanonical cycle. Ann. of Math. (2) 114 (1981), no. $2,267-322$.

[M82] J. Mérindol, Les singularités simples elliptiques, leurs déformations, les surfaces de del Pezzo et les transformations quadratiques. Ann. Sci. École Norm. Sup. (4) 15 (1982), no. 1, 17-44

[Ma89] H. Matsumura, Commutative ring theory, C.U.P., 1989.

[Mum] D. Mumford, An analytic construction of degenerating abelian varieties over complete rings, Compositio Math. 24, (1972), 239-272.

[N80] I. Nakamura, Inoue-Hirzebruch surfaces and a duality of hyperbolic unimodular singularities, Math. Ann. 252 (1980), no. 3, 221-235.

[Ob04] A. Oblomkov, Double affine Hecke algebras of rank 1 and affine cubic surfaces, Int. Math. Res. Not. 2004, no. 18, 877-912.

[P74] H. Pinkham, Deformations of algebraic varieties with $\mathbb{G}_{m}$ action, Astérisque 20, S.M.F., 1974. 
[R83] M. Reid, Decomposition of toric morphisms, in Arithmetic and geometry, Vol. II, 395-418, Progr. Math. 36, Birkhäuser, 1983.

[SYZ96] A. Strominger, S.-T. Yau, and E. Zaslow, Mirror Symmetry is T-duality, Nucl. Phys. B479, (1996) 243-259.

[Ty99] A. Tyurin, Geometric quantization and mirror symmetry, arXiv:math/9902027.

[W76] J. Wahl, Equisingular deformations of normal surface singularities I, Ann. of Math. (2) 104 (1976), no. 2, 325-356.

DPMms, Centre for Mathematical Sciences, Wilberforce Road, Cambridge CB3 OWB, United Kingdom

E-mail address: mgross@dpmms.cam.ac.uk

Department of Mathematics and Statistics, Lederle Graduate Research Tower, University of Massachusetts, Amherst, MA 01003-9305

E-mail address: hacking@math.umass.edu

Department of Mathematics, 1 University Station C1200, Austin, TX 78712-0257

E-mail address: keel@math.utexas.edu 